
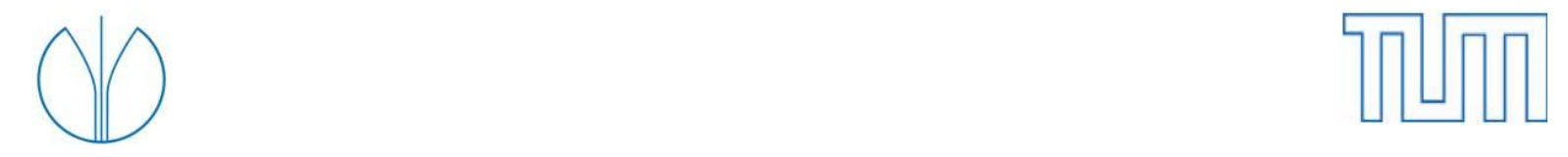

Fakultät Wissenschaftszentrum Weihenstephan für Ernährung, Landnutzung und Umwelt

Lehrstuhl für Biotechnologie der Nutztiere

\title{
Multi-transgenic pigs for xenotransplantation
}

\author{
Konrad Josef Fischer
}

Vollständiger Abdruck der von der Fakultät Wissenschaftszentrum Weihenstephan für Ernährung, Landnutzung und Umwelt der Technischen Universität München zur Erlangung des akademischen Grades

eines Doktors der Naturwissenschaften

genehmigten Dissertation.

Vorsitzender: Prof. Dr. Siegfried Scherer

Prüfer der Dissertation:

1. Prof. Angelika Schnieke, Ph.D.

2. Prof. Dr. Eckhard Wolf

Ludwig-Maximilians-Universität München

3. Prof. Dr. Jochen Seißler

Ludwig-Maximilians-Universität München

Die Dissertation wurde am 15.02.2016 bei der Technischen Universität München eingereicht und durch die Fakultät Wissenschaftszentrum Weihenstephan für Ernährung, Landnutzung und Umwelt am 21.06.2016 angenommen. 


\section{Inhalt}

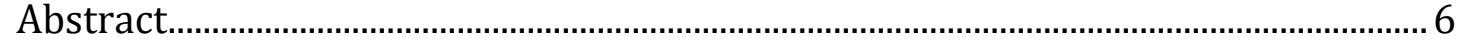

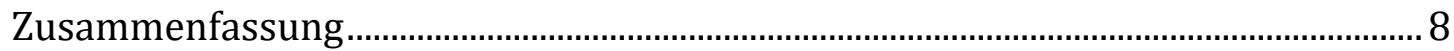

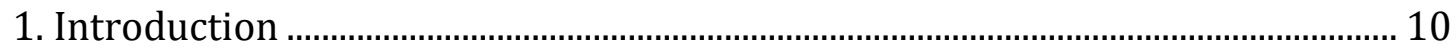

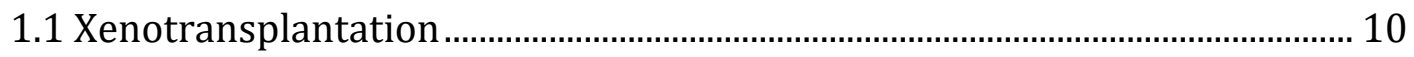

1.2 Hyperacute Rejection ................................................................................. 11

1.3 Acute vascular and acute humoral xenograft rejection ................................... 13

1.4 Complement regulators.................................................................................. 14

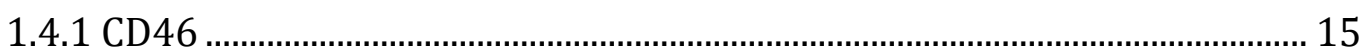

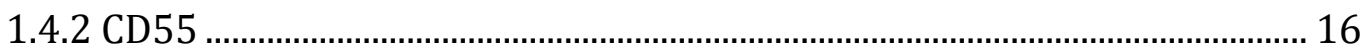

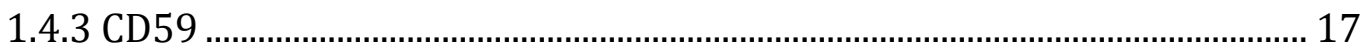

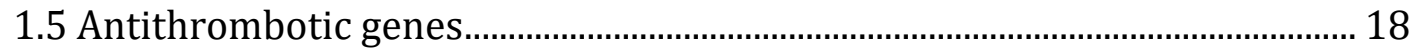

1.6 Endothelium protective genes............................................................................ 20

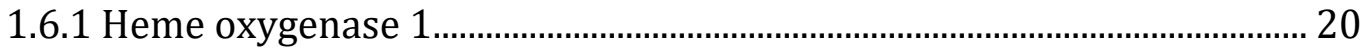

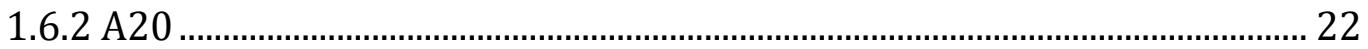

1.7 Immune regulatory genes and cell mediated xenograft rejection................. 24

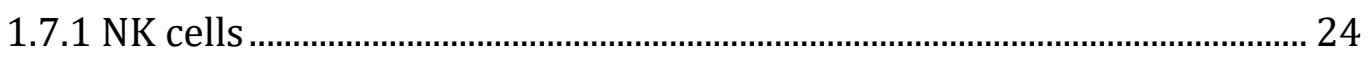

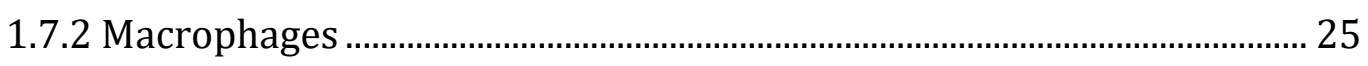

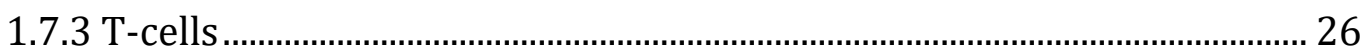

1.8 BAC and PAC vectors ......................................................................................... 27

1.9 Human artificial chromosomes and MMCT ...................................................... 28

1.10 Genetic modifications in livestock.................................................................. 30

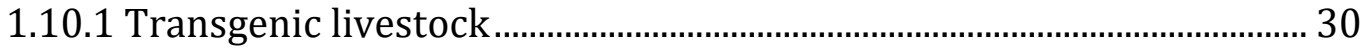

1.10.2 Precise genetic modifications ………………………………………... 31

1.11 Porcine ROSA26 6.............................................................................................. 34

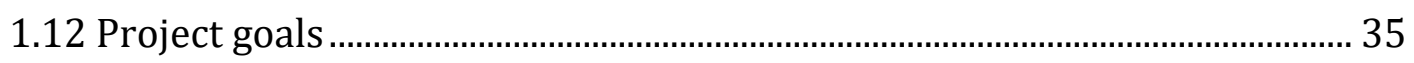

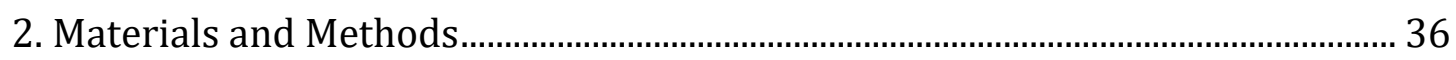

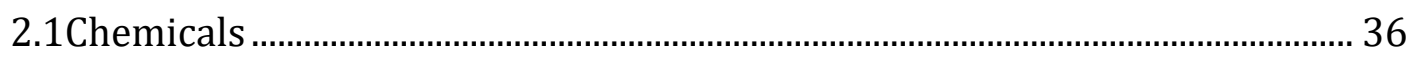

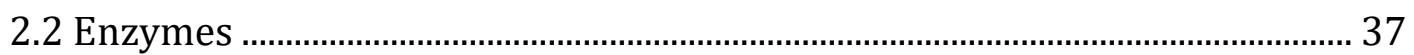

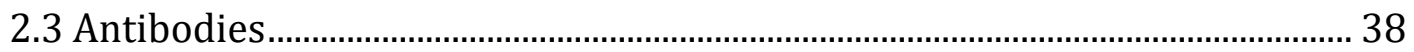

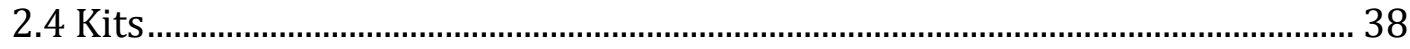

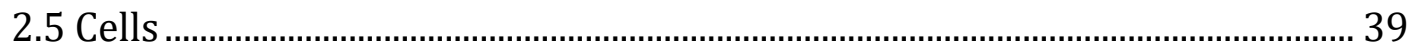

2.5.1 Bacterial strains ...................................................................................... 39

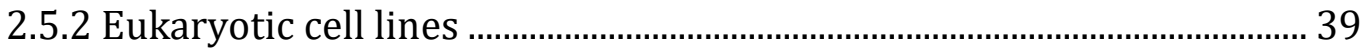

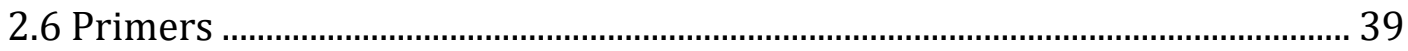

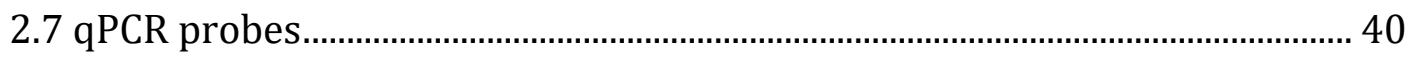




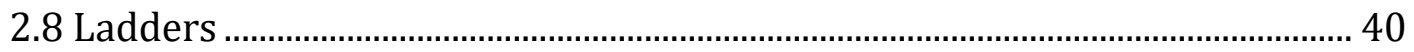

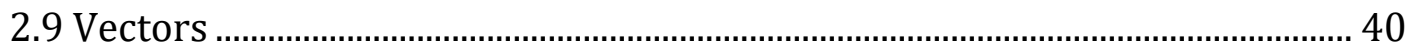

2.9.1 Bacterial Artificial Chromosomes ……........................................................... 40

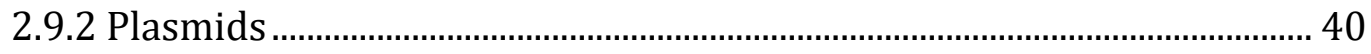

2.10 Tissue culture media and supplements.......................................................... 41

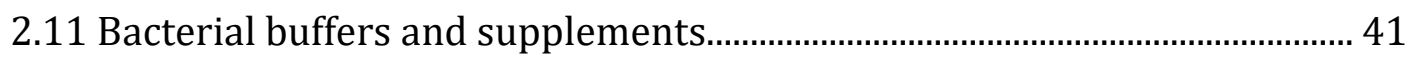

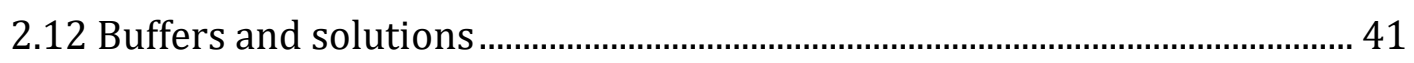

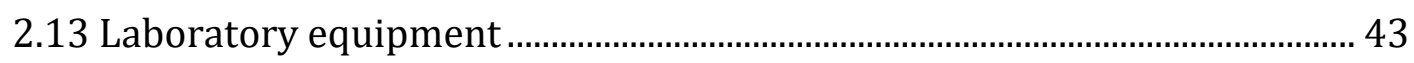

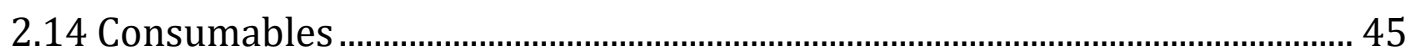

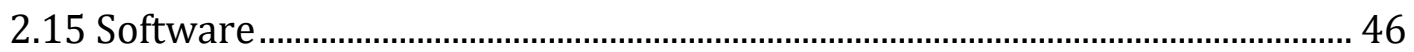

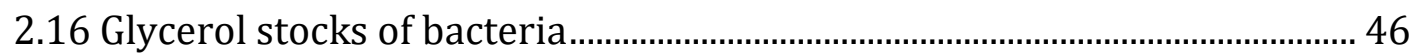

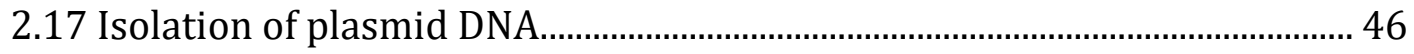

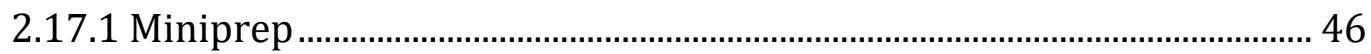

2.17.2 Midiprep and Maxiprep........................................................................... 47

2.18 Recombineering competent SW106 …............................................................. 47

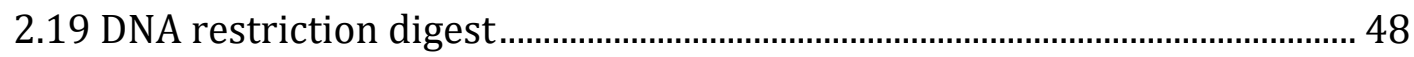

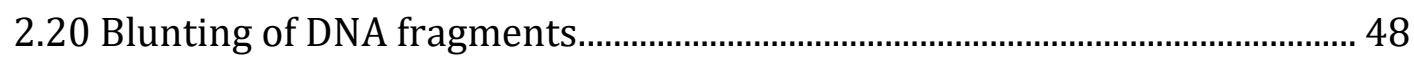

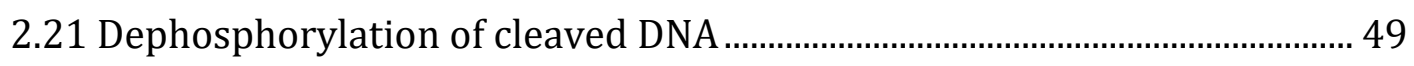

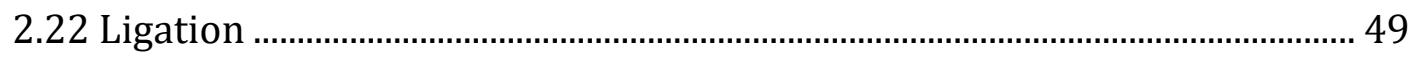

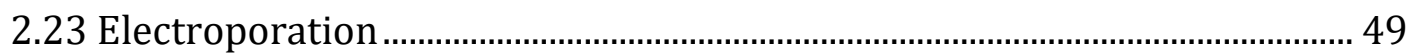

2.24 Colony PCR

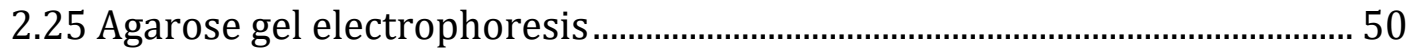

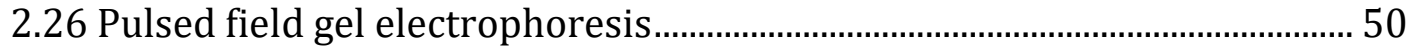

2.27 Isolation of DNA fragments from agarose-gels.............................................. 51

2.28 Determination of DNA and RNA concentration............................................ 51

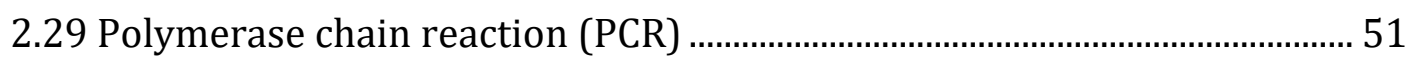

2.30 Splice Overlap Extension PCR (SOE-PCR) ……............................................ 52

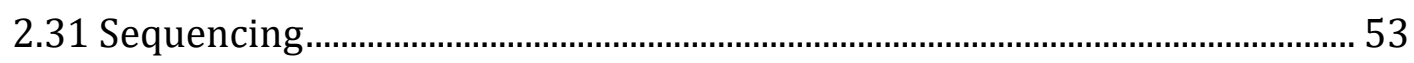

2.32 Microcell-mediated chromosome transfer..................................................... 53

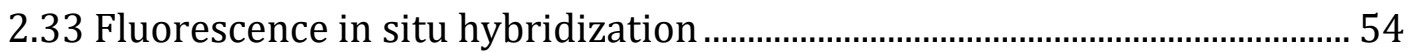

2.33.1 Preparation of metaphase chromosome spreads .................................. 54

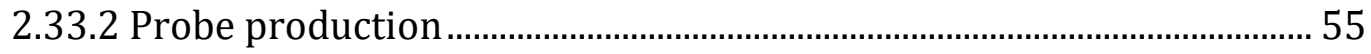

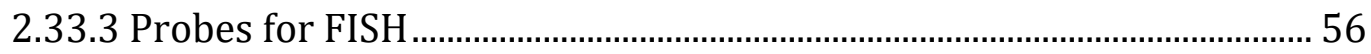

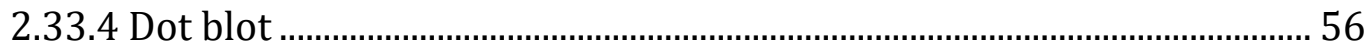

2.33.5 Probe precipitation and denaturation ...................................................... 57

2.33.6 RNase treatment and pepsin digestion ................................................... 57 
2.33.7 Probe hybridization .................................................................................... 58

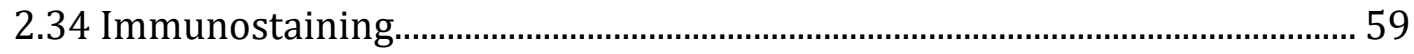

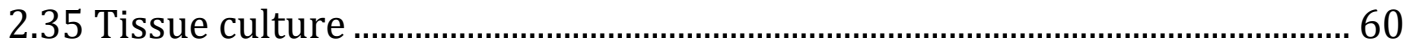

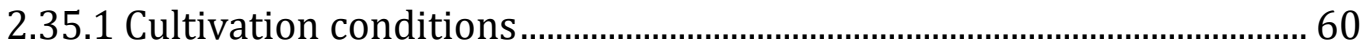

2.35.2 Cryoconservation...................................................................................... 60

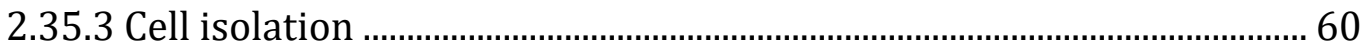

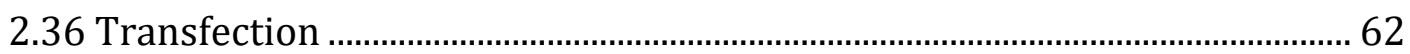

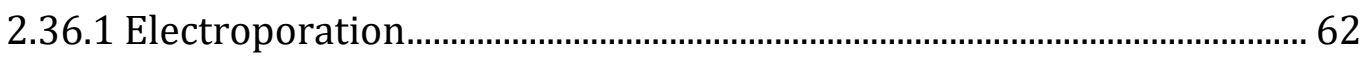

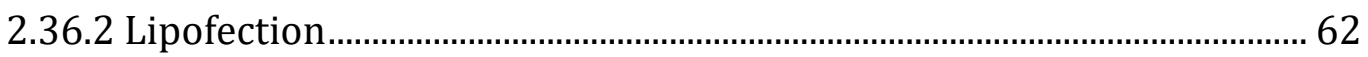

2.37 Selection of stably transfected clones ................................................................. 63

2.38 Isolation of mammalian genomic DNA and RNA ........................................... 63

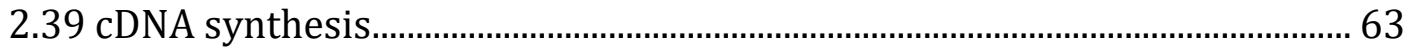

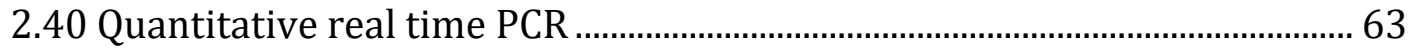

2.41 Protein isolation of mammalian cells ................................................................. 64

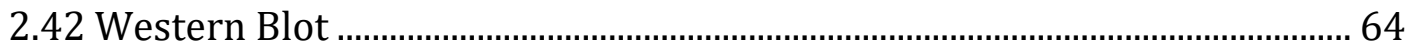

2.43 ZFN-mediated GGTA1 gene inactivation ......................................................... 65

2.44 Immunofluorescence staining and flow cytometry ........................................ 65

2.45 Assay for complement-mediated lysis of porcine fibroblasts........................ 66

2.46 Cytokine-induced upregulation of E-selectin and MHC class-II................... 66

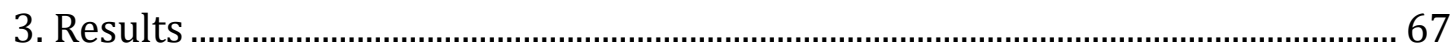

3.1 Generation of xeno-gene expression vectors..................................................... 68

3.1.1 Resistance cassettes for pro- and eukaryotic cells ...................................... 68

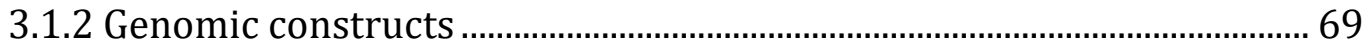

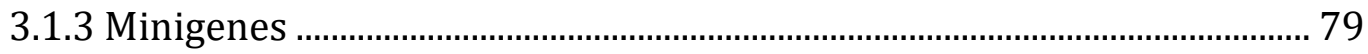

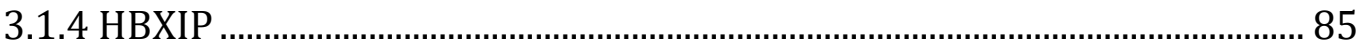

3.2 Xeno-HAC formation and transfer.................................................................. 88

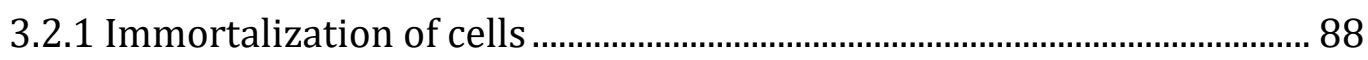

3.2.2 HAC transfer using MMCT....................................................................... 90

3.2.3 Formation of xeno-HAC.............................................................................. 94

3.3 Multi transgenic cells and animals...................................................................... 96

3.3.1 Generation of multi-transgenic porcine cells ............................................ 96

3.3.2 Production and analysis of multi-transgenic pigs .................................... 98

3.3.3 GGTA1 knockout in 1706 cells....................................................................102

3.4 Xeno-gene placement at the porcine ROSA26 locus .......................................107

3.4.1 ROSA26 targeting and the generation of cloned piglets ..........................107

4. Discussion ................................................................................................................... 
4.1 Modification of the CD55 BAC construct

4.2 Functions of CD55

4.3 A wild type CD55 cDNA or delta-SCR1 cDNA mutant for the production of transgenic animals?..

4.4 A CD55 minigene for the production of transgenic animals. 116

4.5 The CD46 minigene. 117

4.6 The CD39 cDNA construct. 118

4.7 Immortalization of cells using hTERT .

4.8 Human artificial chromosomes and microcell mediated chromosome transfer

4.9 ROSA26 targeting and expression of HO1

4.10 Multi-transgenic animals 125

5. Summary and outlook 128

6. Abbreviations 129

7. List of Tables 131

8. List of Figures 131

9. Literature. 133

10. Supplementay part: Primer sequences 155

11. Acknowledgement. 157

12. Curriculum Vitae .159 


\section{Abstract}

Efficient clinical xenotransplantation requires multi-modified pigs that coexpress several xenoprotective transgenes and carry inactivation mutations in several endogenous genes. Great progress has been achieved in recent years in identifying means of inhibiting the hyperacute and to some extent the acute vascular rejection. The main hurdle at the moment is to obtain high and ubiquitous expression of all complement regulatory genes and to combine all the necessary modifications into a single genotype.

In this work different approaches are shown how several xenoprotective transgenes can be combined at one genomic locus. One approach is based on the use of a large bacterial artificial chromosome (BAC) construct, carrying the genomic sequence of the complement regulatory gene human CD55 onto which further coagulation regulatory (human thrombomodulin) and T-cell regulatory genes (LEA29Y) or anti-apoptotic and anti-inflammatory genes such as human A20 and human heme oxygenase 1 (HO1) were added. This approach allows cotransfection of several xenogenes but does not allow predetermination of the genomic integration site(s).

The constructs made in this work were subsequently used for cotransfection experiments together with BAC or PAC constructs carrying the genomic sequence of CD46 or CD59 to obtain either a human artificial chromosome (HAC) that carries several xenoprotective transgenes or multi-transgenic cells that express a battery of xenoprotective transgenes.

Although it was not possible to create a functional HAC, a preexisting HAC could be successfully transferred via microcell mediated chromosome transfer from human into porcine cells. This method could in principle be used to transfer whole human chromosomal loci encoding xenoprotective genes into porcine cells.

However, the cotransfection experiments delivered a large number of cell clones expressing a wide range of xenoprotective transgenes. Cells revealing the highest expression levels of the complement regulatory genes were used for somatic cell nuclear transfer and two founder animals were obtained. One animal revealed CD46 and CD55 expression and one multi-transgenic founder animal expressed CD46, CD55, CD59, H01 and A20. 
Analysis of different tissues showed expression of the transgenes in almost all organs. Moreover, a complement-mediated lysis assay revealed complete protection of the five-fold transgenic cells. Subsequently, these cells were further modified by a knockout of the GGTA1 gene.

An additional approach to obtain transgenic animals suitable for xenotransplantation was based on targeted placement of a HO1 construct at the permissive porcine ROSA26 locus. A transgenic animal was obtained via somatic cell nuclear transfer which showed ubiquitous expression of H01. To investigate whether further transgenes could be added at the same locus by 'gene-stacking', a retargeting vector was generated to place a CAG-driven CD55 minigene adjacent to H01. By this approach several xenogenes can be precisely placed "step by step" at a single genomic locus known to support expression.

This is one of the first examples showing that efficient placement of several xenogenes can be achieved and how multi-modified pigs can be generated in a single step. Moreover, preselection of cells resulted in animals revealing expression levels which exceed the best published results. The approach used avoids segregation of transgenes during breeding, thereby drastically reducing the number of experimental animals required. Together with the knockout of the major xeno antigen, organs from these animals will bring xenotransplantation closer to the clinic. 


\section{Zusammenfassung}

Für eine effiziente klinische Xenotransplantation werden mehrfach transgene Schweine benötigt, die sowohl protektive Xenogene exprimieren als auch eine Inaktivierung von mehreren porzinen Genen aufweisen. In den letzten Jahren wurde ein großer Fortschritt darin erzielt, die hyperakute Abstoßungsreaktion sowie auch teilweise die akut vaskuläre Abstoßungsreaktion zu verhindern. Die Hauptaufgabe besteht zurzeit darin, eine hohe und ubiquitäre Expression aller komplement-regulatorischen Gene zu erhalten und alle Modifikationen in einem einzigen Schwein zusammenzufassen.

In dieser Arbeit werden verschiedene Ansätze beschrieben, wie mehrere protektive Xenogene an einem genomischen Lokus kombiniert werden können. Eine dieser Möglichkeiten besteht in der Verwendung eines großen künstlichen Bakterienchromosoms (BAC), das die genomische Sequenz des komplementregulatorischen Gens CD55 trägt und worauf weitere Gene wie das humane, blutgerinnungs-hemmende Gen Thrombomodulin und das T-Zell-regulatorische Gen LEA29Y bzw. die humanen anti-apoptotischen sowie antiinflammatorischen Gene A20 und Häm-oxygenase 1 (HO1) hinzugefügt wurden. Durch diesen Ansatz können zwar mehrere Xenogene gleichzeitig transfiziert werden, jedoch besteht keine Möglichkeit die chromosomale Integrationsstelle vorab festzulegen.

Die in dieser Arbeit hergestellten Konstrukte wurden zusammen mit weiteren BAC bzw. PAC Konstrukten, welche die genomische Sequenz von humanem CD46 oder humanem CD59 trugen, transfiziert um einerseits künstliche humane Chromosomen (HAC) zu erzeugen, die eine Vielzahl an xeno-protektiven Genen tragen oder multi-transgene Zellen zu erzeugen, die eine große Anzahl an xenoprotektiven Genen gleichzeitig exprimieren.

Obwohl die Neubildung eines HACs nicht erreicht werden konnte, so konnte ein bereits bestehendes HAC durch den mikrozellen-vermittelten Chromosomentransfer erfolgreich von humanen in porzine Zellen übertragen werden. Diese Methode kann grundsätzlich auch dazu verwendet werden um komplette menschliche Chromosomen, die eine Vielzahl von xeno-protektiven Genen enthalten, in porzine Zellen zu übertragen. 
Jedoch lieferten die Kotransfektionsexperimente eine große Anzahl an Zellklonen, die mehrere xeno-protektive Gene gleichzeitig exprimierten. Zellen mit den höchsten Expressionsniveaus der komplement-regulatorischen Gene wurden für den somatischen Kerntransfer verwendet. Daraus gingen zwei Gründertiere hervor, die eine hohe CD46 und CD55 Expression aufwiesen bzw. ein multi-transgenes Gründertier mit einer Expression von CD46, CD55, CD59, H01 und A20. Die Analyse unterschiedlicher Gewebe zeigte die Expression der Transgene in fast allen Organen. Zusätzlich zeigte ein Test mit humanem Serum den vollständigen Schutz der multi-transgenen Zellen vor der komplementvermittelten Zelllyse. Diese Zellen wurden darauffolgend durch eine Inaktivierung des GGTA1 Gens weiter modifiziert.

Ein weiterer Ansatz um transgene Tiere $\mathrm{zu}$ erhalten, die für die Xenotransplantation geeignet sind, bestand aus einer zielgerichteten Platzierung eines H01 Konstruktes in dem gut zugänglichen porzinen ROSA26 Lokus. Über den somatischen Kerntransfer wurde ein transgenes Tier erzeugt, das eine ubiquitäre Expression von H01 aufwies. Um herauszufinden, ob weitere Transgene am gleichen Lokus platziert werden könnten, wurde ein weiterer Vektor kloniert, der es erlaubt ein CAG-CD55 Minigen neben H01 zu platzieren. Durch diesen Ansatz können mehrere Xenogene präzise nacheinander an einem einzigen genomischen Lokus platziert werden der eine Expression dieser Gene erlaubt.

Dies ist eines der ersten Beispiele welches zeigt, dass mehrere Xenogene sehr effizient an einem Lokus platziert und wie multi-modifizierte Schweine in einem einzigen Schritt erzeugt werden können. Durch die Vorauswahl der Zellen konnten Tiere geschaffen werden, deren Expressionslevel die besten veröffentlichen Ergebnisse übertreffen. Der verwendete Ansatz verhindert die Segregation der Transgene während der Züchtung wodurch sich die Anzahl der Versuchstiere drastisch verringert. In Verbindung mit der Inaktivierung des wichtigsten Xenoantigens, werden die Organe dieser Tiere die Xenotransplantation näher an die klinische Umsetzung heran bringen. 


\section{Introduction}

\subsection{Xenotransplantation}

The number of patients with end-stage organ failure is steadily increasing. The only effective clinical therapy remains organ transplantation. However, the number of allogeneic donor organs is limited. In January 2014, 15292 people were on the eurotransplant waiting list for an organ, but only 6866 organs were transplanted in 2013 (www.eurotransplant.org). The severe world-wide shortage of organs from human donors has motivated research into xenotransplantation as a possible alternative.

Xenotransplantation is the transfer of living cells, tissues or organs across species. Non-human primates have been considered as a source for xenotransplantation, but their numbers are very limited. Moreover, the use of primates presents serious ethical concerns and a high risk of cross-species disease transmission (Michler, 1996).

A practical alternative is to use pigs. Many pig organs are similar in size and structure to those of humans, and pigs are widely raised as a food source. Breeding and production techniques are thus well established and their use as donors raises fewer ethical problems. Pigs reproduce very quickly with rapid sexual maturation and large litter sizes. The risk of zoonotic transmission is much lower than primates due to their greater phylogenetic distance from humans (Dooleniya and Warrens, 2003; Cozzi et al., 2009). Donor animals can be systematically selected on the basis of blood group, compatible SLA type and the lack of infectious pathogens.

Pigs can easily be raised in a designated pathogen free (DPF) facility and be thoroughly examined for infectious exogenous diseases before use as donors (Mueller et al., 2011). Porcine endogenous retroviruses (PERV-A, B and C) have been intensively investigated because they integrate into the germ line and are transmitted vertically to offspring. The actual risk posed to humans by these proviruses is unclear, but there is consensus that they should be avoided if possible. PERV-A and PERV-B are present in almost all pigs and can infect human cells (Dieckhoff et al., 2008). 
Pigs free of active PERV-C are most suitable for xenotransplantation because this avoids possible recombination between PERV-A and PERV-C, resulting in PERV$\mathrm{A} / \mathrm{C}$, which has been reported to produce infectious particles (Bartosch et al., 2004). To reduce the risk of PERV transmission to humans, pigs with minimal PERV-A and PERV-B expression levels have to be selected. Additionally, different strategies have been developed to inhibit PERV transmission including vaccines or inhibition of PERV expression using PERV-specific short hairpin RNA (shRNA) (Denner, 2008).

However, animals completely free of active PERVs have been sourced and raised by the company Living Cell Technologies (LCT) in New Zealand. These animals are well characterized regarding the status of almost all transmittable endogenous, viral or bacterial pathogens and have already been used for clinical trials of microencapsulated porcine pancreatic islets with a group of fourteen patients with severe unaware hypoglycaemia (Garkavenko et al., 2012).

\subsection{Hyperacute Rejection}

Transplantation of a porcine organ into a human recipient invokes several rejection responses. The first and most severe is hyperacute rejection (HAR) (Fig. 1). In HAR, pre-formed antibodies against the $\alpha 1,3$-galactosyl-galactose ( $\alpha \mathrm{Gal}$ ) epitope on the surface of porcine endothelial cells activate the human complement system leading to rapid destruction of the xenograft within minutes or hours (Oriol et al., 1993; Good et al., 1992; Cooper at al., 1994). The $\alpha \mathrm{Gal}$ epitope is synthesised by the gene GGTA1 ( $\alpha 1,3$-galactosyltransferase) which is active in almost all mammals but not in old world primates, including humans. Humans thus lack endogenous $\alpha \mathrm{Gal}$ antigens, and because $\alpha \mathrm{Gal}$ is expressed by some microbes we are permanently exposed and have high levels of preformed natural IgG, IgM and IgA antibodies (1\% of total serum immunoglobulin). Recognition of porcine $\alpha \mathrm{Gal}$ epitopes thus leads to rapid xenograft destruction (Galili et al., 1985; Galili, 1993). 


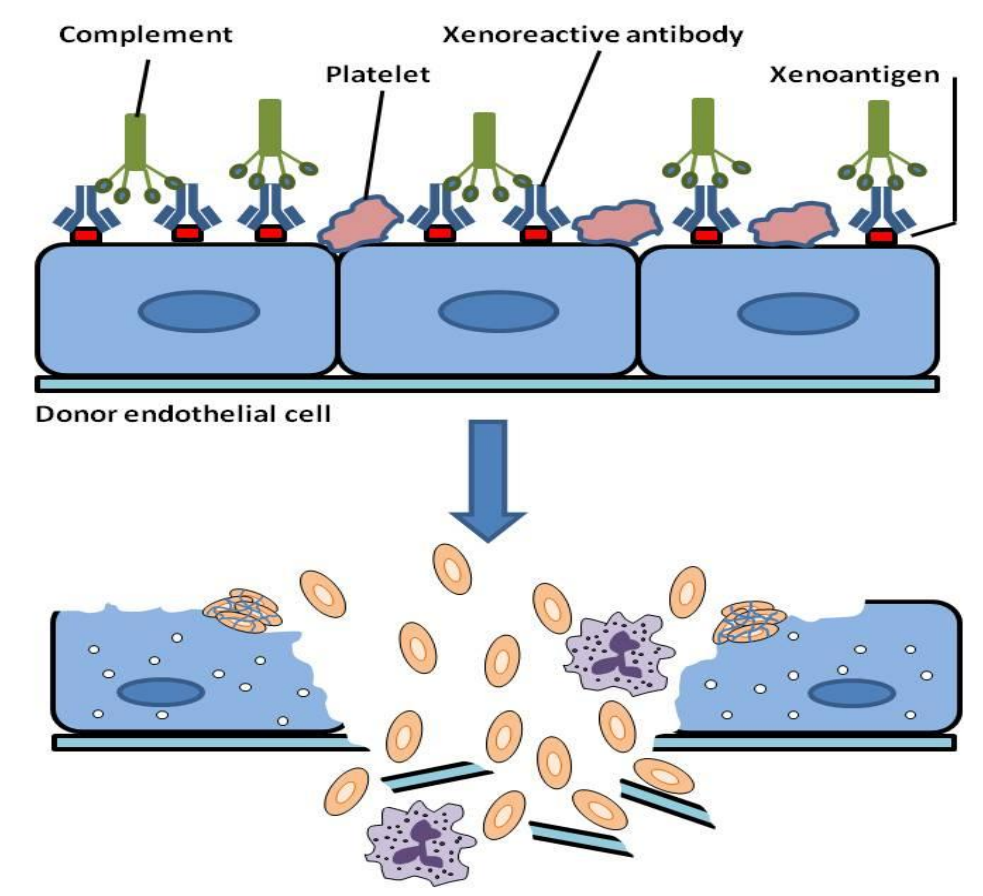

Figure 1. Hyperacute rejection after xenotransplantation causing complement activation and graft rejection (adapted from Yang and Sykes, 2007).

Efforts have been made to reduce the quantity of $\alpha \mathrm{Gal}$ epitopes on porcine tissues by overexpression of human $\alpha 1,2$-fucosyltransferase (H-transferase) which competes with $\alpha 1,3$-galactosyltransferase for the same substrate $\mathrm{N}$ acetyllactosamine. This strategy reduced the amount of $\alpha$-Gal epitopes but some $\alpha$-Gal epitopes remained and hyperacute xenograft rejection still occurred (Sandrin et al., 1995; Jeong et al., 2013).

Attempts to reduce anti-Gal antibodies via in vivo immunoadsorption using specific Gal- $\alpha 1,3-$ Gal columns were able to hold off hyperacute rejection for approximately one week, but this approach was not effective in the longer term (Taniguchi et al., 1996).

Several groups have reported inactivation of the GGTA1 gene. Dai et al., 2002 and Lai et al., 2002 reported such a 'knockout' of one GGTA1 allele. Phelps et al., 2003 and Kolber-Simonds et al., 2004 subsequently achieved homozygous GGTA1 knockout. These animals provided the most significant advance in avoiding HAR. GGTA1-deficient pig hearts transplanted heterotopically into baboons did not undergo hyperacute rejection and survived for 2-6 months (Kuwaki et al., 2005), but these animals did however undergo acute vascular and acute humoral xenograft rejection. 


\subsection{Acute vascular and acute humoral xenograft rejection}

Acute vascular rejection (AVR) and acute humoral xenograft rejection (AHXR) begin approximately one day after xenotransplantation and gradually destroy the xenograft (Platt, 1998).

AVR and AHXR are probably initiated by xenoreactive antibodies that cause activation of the graft endothelium. AVR leads to activation of the complement system and the coagulation system and results in apoptosis, thrombosis, oedema and platelet aggregation in the graft (Platt et al., 1991). AHXR is characterized by cellular infiltration of neutrophils, CD8+ T cells, NK cells and macrophages (Fig. 2) (Vega et al., 2002).

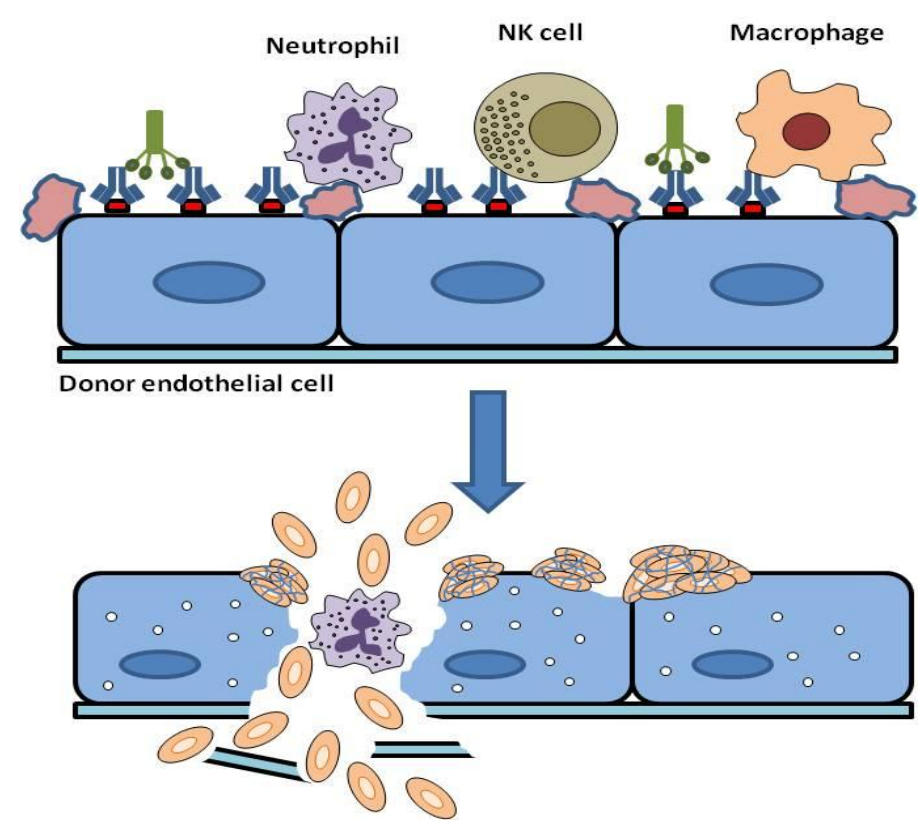

Figure 2. Acute vascular rejection causes graft destruction by complement activation, endothelial cell activation and antibody-dependent cell-mediated cytotoxicity (adapted from Yang and Sykes, 2007).

Most xeno-reactive non-Gal antibodies are directed against Hanganutziu-Deicher (H-D) antigens, a group of glycoproteins and gangliosides containing $\mathrm{N}$ glycolylneuraminic acid (Higashi et al., 1977; Merrick et al., 1978; Morozumi et al., 1999). One approach to inhibiting AVR and AHXR, is to inactivate the genes responsible for H-D antigen synthesis, notably the CMAH (cytidine monophospho- $\mathrm{N}$-acetylneuraminic acid hydroxylase) gene.

The CMAH enzyme converts $\mathrm{N}$-acetylneuraminic acid (Neu5Ac) into $\mathrm{N}$ glycolylneuraminic acid (Neu5Gc). 
All mammals and non-human primates have a functional CMAH gene and produce Neu5Gc. However, the human CMAH gene has undergone a $92 \mathrm{bp}$ deletion in exon 6, which results in a non-functional CMAH protein (Varki, 2001). Humans are exposed to Neu5Gc epitopes from food sources and these result in high levels of anti-Neu5Gc antibodies (0.1-0.2\% of human IgGs) that induce complement activation and lysis of porcine endothelial cells (Bardor et al., 2005; Nguyen et al., 2005; Baumann et al., 2007; Saethre et al., 2007).

Double knockout of the porcine GGTA1 and CMAH genes using zinc-finger nucleases has recently been reported by Lutz et al., 2013. Antibody-binding and antibody-mediated complement-dependent cytotoxicity assays found reduced immunological reactivity in these double knockout cells.

\subsection{Complement regulators}

The complement system consists of more than 30 proteins and is a major defense mechanism against the invasion of pathogens. It is part of the innate immune system and can be activated by the classical-, alternative- and lectin pathways (Fig. 3).

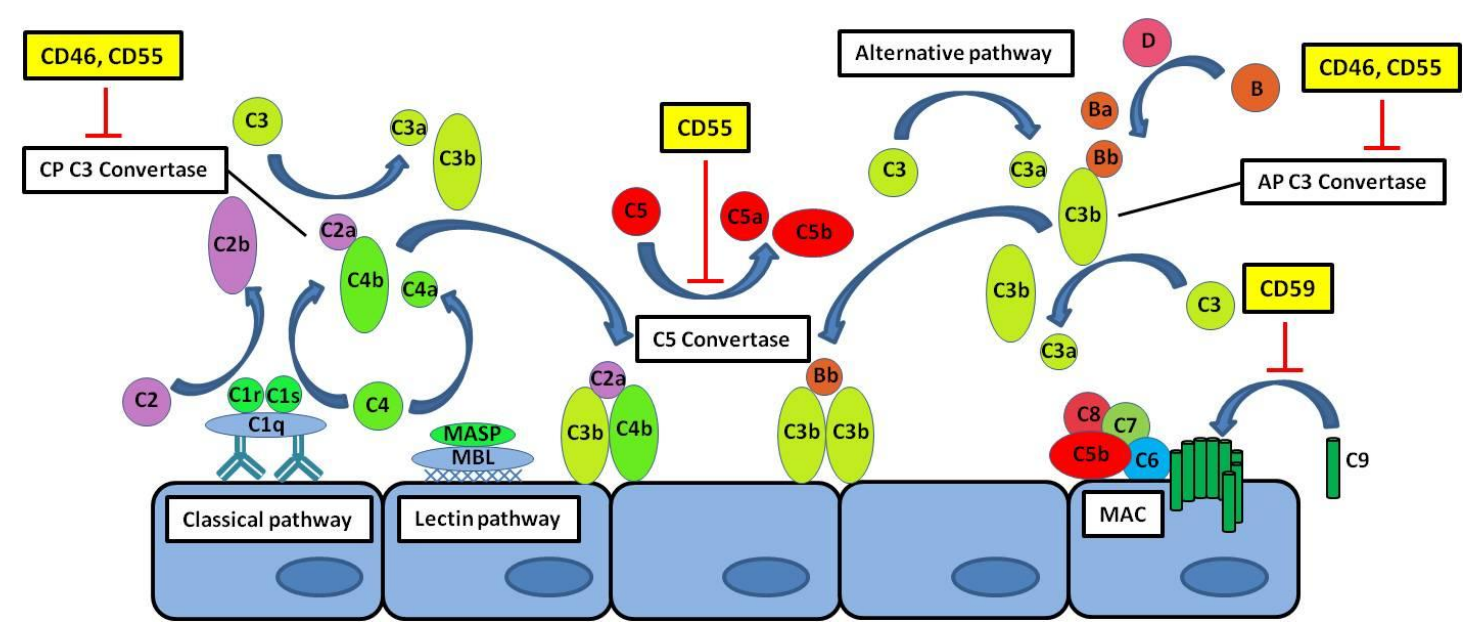

Figure 3. Pathways of the complement system and interaction with the complement regulatory genes CD46, CD55 and CD59 (adapted from Ricklin and Lambris, 2007).

It is tightly controlled at several levels and the complement regulators CD46, CD55 and CD59 play an important role in inhibiting complement activation. These proteins are thus useful in preventing xenograft rejection. 
Complement regulatory proteins (CRPs) are species-specific and thus ineffective across species boundaries (Miyagawa et al., 1988). To block the human complement system, it is necessary to express human CRPs in porcine cells. Effective inhibition of complement activation also requires a combination of different CRPs and abundant expression (Miyagawa et al., 2010).

\subsubsection{CD46}

Membrane cofactor protein (MCP or CD46) inhibits C3 convertase activation by acting as a cofactor for plasma serine protease factor-I-mediated C3b and C4b inactivation (Seya et al., 1986). The human CD46 gene consists of 14 exons and has a size over $40 \mathrm{~kb}$ (Liszewski et al., 1991). The $\mathrm{C} 3 \mathrm{~b} / \mathrm{C} 4 \mathrm{~b}$ binding region is encoded by exons 2-6. Alternative splicing of exons 7-14 results in multiple membrane-bound and soluble isoforms (Purcell et al., 1991; Post et al., 1991). In humans, 20 different isoforms of CD46 are known, of which 13 are protein coding (www.ensembl.org). In mammals, mice, rats and guinea pigs, several other CD46 isoforms have been identified (Hosokawa et al., 1996; Pollard et al., 1998; Miwa et al., 1998; Nomura et al., 1999; Tsujimura et al., 2001). The biological functions of all isoforms have not yet been determined, but based on in vitro experiments, some isoforms seem to preferentially inhibit the classical pathway of complement activation whereas others inhibit the alternative pathway (Liszewski et al., 1996). The CD46 protein is expressed at high level by almost all nucleated cells (McNearney et al., 1989; Johnstone et al., 1993). Several groups have tried to create transgenic pigs or mice with high CD46 expression using minigenes or cDNA constructs (Yannoutsos et al., 1995; Mulder et al., 1997; Mora et al., 1996; Miyagawa et al., 1997), but achieving ubiquitous high expression has been very difficult. One pig line has however been reported as expressing abundant and ubiquitous expression of CD46, from a minigene, and this prevented hyperacute kidney rejection in non-immunosuppressed baboons for several days even without GGTA1 inactivation (Loveland et al., 2004). 


\subsubsection{CD55}

Decay-accelerating factor (DAF or CD55) protects cells from complementdependent lysis by accelerating the decay of C3 and C5 convertases (Medof et al., 1984). The human CD55 gene is approximately $40 \mathrm{~kb}$ and expresses thirteen RNA splicing variants, nine of which are translated into functional protein isoforms (www.ensembl.org). The most important isoforms in humans are the glycosyl-phosphatidylinositol (GPI) anchored CD55 (gDAF) and the soluble CD55 (sDAF). gDAF is composed of four $\mathrm{N}$-terminal complement control protein domains (CCP), a heavily O-glycosylated serine, threonine and proline (STP)-rich domain and a C-terminal GPI-anchored domain (Coyne et al., 1992). This isoform is expressed on the cell membranes of all blood cells and almost all cell types that come into close contact with plasma component proteins. sDAF is less abundant than gDAF and is present in body fluids and the extracellular matrix (Medof et al., 1987). The sDAF molecule is formed by alternative splicing resulting in insertion of exon 10, which causes a translational frameshift in exon 14 leading to the lack of the GPI-anchor (Caras et al., 1989). Several other isoforms of CD55 with alternatively spliced exons and a modified STP-rich domain have been identified in guinea pig (Nonaka et al., 1998; Wang et al., 1998).

Miyagawa et al., (2006) showed that expression of CD55 at least 5-fold greater than the human endogenous level is necessary to efficiently inhibit complement activation. Regulation of CD55 expression is not completely understood, but is known to be controlled by several cytokines, including tumor necrosis factor $\alpha$ (TNF- $\alpha$ ) (Manson et al., 1999; Ahmad et al., 2003), transforming growth factor $\beta$ (TGF- $\beta$ ) (Cocuzzi et al., 2001) and interferon $\gamma$ (IFN- $\gamma$ ) (Manson et al. 1999) and upregulated by vascular endothelial growth factor and basic fibroblast growth factor (Manson et al., 2004; Manson et al., 2002). In colon cancer cells, prostaglandin E2 ( $\left.\mathrm{PGE}_{2}\right)$ increased CD55 expression (Holla et al., 2005). The human CD55 promoter sequence contains a SMAD-binding site, and interactions of CD55 with SMADs, dysferlin and myostatin have been investigated by Wenzel et al., (2005). 
Several groups have produced transgenic CD55 pig lines. Cozzi et al., (1997) made several transgenic pigs using a $6.5 \mathrm{~kb}$ CD55 minigene. Byrne et al., (1997) produced CD55 and CD59 transgenic pigs and Jeong et al., (2013) generated transgenic pigs expressing CD55, CD59 and H-transferase. Although none of these achieved high and ubiquitous CD55 expression, they have demonstrated effective inhibition of complement activation and showed the usefulness of combining different complement regulatory proteins in extending graft survival in pig-to-primate organ transplantation.

\subsubsection{CD59}

CD59 (protectin) is a ubiquitously expressed membrane-bound glycoprotein that binds the complement proteins C8 and C9 in the activated membrane attack complex (MAC). It prevents full assembly of the MAC by inhibiting the incorporation of additional C9 copies into the complex, thus blocking cell lysis (Bubeck et al., 2011; Walport, 2001). The genomic CD59 sequence is $34 \mathrm{~kb}$ in size and contains 7 exons (www.ensemble.org). The CD59 gene expresses 14 RNA splicing variants which mainly vary in their $5^{\prime}$ and $3^{\prime}$ UTRs, but almost all encode the same 128 amino acid protein. The molecular mass of CD59 varies between 18 and $22 \mathrm{kDa}$, depending on the level of $\mathrm{N}$ - or O-glycosylation (Stefanova et al., 1989; Hadam, 1989; Groux et al., 1989). The structure of the CD59 protein consists of a hydrophobic signal sequence, a cysteine-rich sequence and a hydrophobic C-terminal to which a glycosyl-phosphatidylinositol anchor is attached after translation (Davies et al., 1989; Sawada et al., 1990; Philbrick et al., 1990).

Several CD59 transgenic pig lines have been reported. Niemann et al., (2001) produced pigs with CD59 cDNA driven by the CMV promoter that express at high level in heart, kidney, skeletal muscle, skin and pancreas and demonstrated protection against hyperacute rejection and complement activation by ex vivo perfusion of kidneys with human blood. However CD59 expression varied considerably between the offspring. 
Chen et al., (1999) generated CD55, CD59 double transgenic pigs. Porcine hearts were transplanted into immunosuppressed baboons and showed prolonged survival, 85-130 hours compared to 20-80 minutes with wild type. Deppenmeier et al., (2006) investigated the health of CD59 transgenic pigs. Nineteen CD59 transgenic pigs from 9 different lines were examined between 1 to 32 months old and no pathomorphological phenotype was observed related to the presence of the transgene.

\subsection{Antithrombotic genes}

A combination of complement regulatory transgenes and GGTA1 gene inactivation can effectively control hyperacute rejection (Van Denderen et al., 1997; Cowan et al., 1998; McGregor et al., 2012). Although the complement system and coagulation system are closely connected (Amara et al., 2010), additional genes regulating the coagulation system are necessary to inhibit AVR and AHXR. Because porcine coagulation factors are unable to control the human coagulation system, it is also necessary to express human versions of antithrombotic genes in porcine xenografts.

The porcine tissue factor pathway inhibitor (TFPI) cannot inactivate human factor $\mathrm{Xa}$ and inhibit activation of prothrombin to thrombin. Porcine thrombomodulin can bind human thrombin, but this complex is unable to activate protein C (Siegel et al., 1997; Roussel et al., 2008).

Thrombomodulin (TM) is a transmembrane endothelial cell glycoprotein receptor that plays an important role in modulating inflammation, coagulation and fibrinolysis. TM itself has several anti-inflammatory properties by sequestering pro-inflammatory molecules such as the high-mobility-groupprotein B1 (HMGB1) (Abeyama et al., 2005), or inhibiting the adhesion of neutrophils to endothelial cells (Conway et al., 2002). 


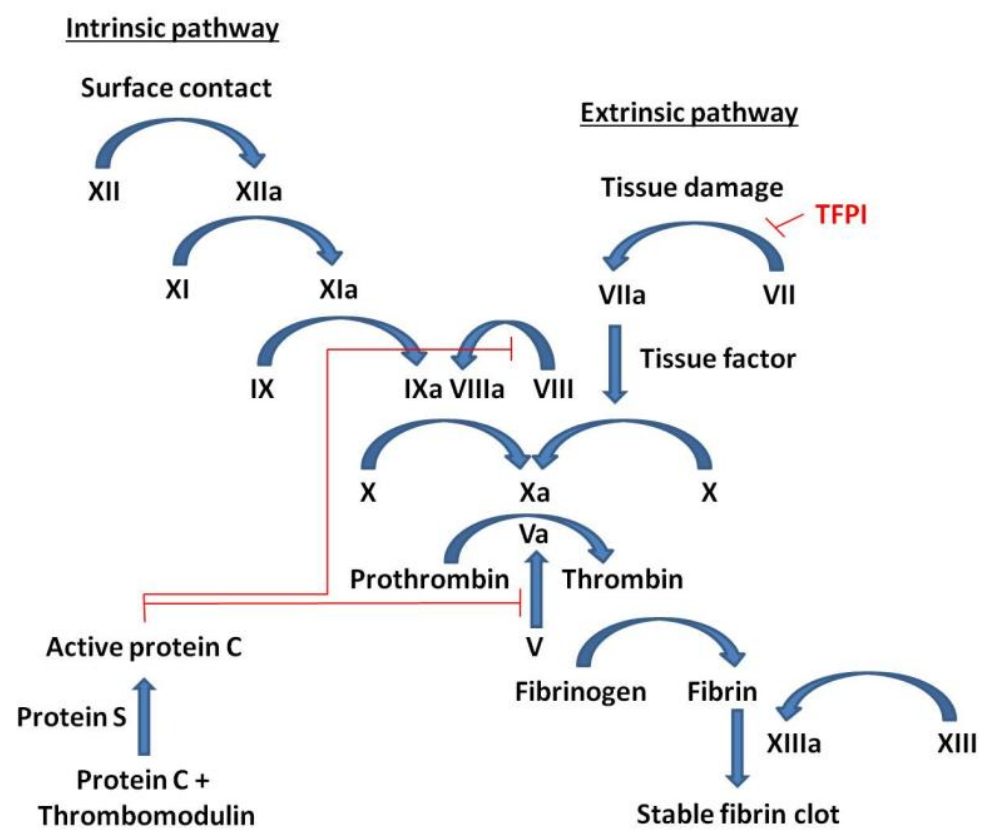

Figure 4. Pathways of blood coagulation.

Thrombomodulin acts as a cofactor for thrombin (Fig. 4). If a TM-thrombin complex is formed, thrombin is no longer able to cleave fibrinogen into fibrin and initiate thrombosis. The TM-thrombin complex cleaves and activates circulating protein C, a vitamin K-dependent glycoprotein (Esmon, 1987). This activation can be increased up to 20 fold by the endothelial protein $\mathrm{C}$ receptor, a transmembrane protein that concentrates protein $\mathrm{C}$ at the endothelial cell surface (Taylor et al., 2001). Activated protein C inactivates coagulation factors Va and VIIIa and suppresses further thrombin formation (Suzuki et al., 1983; Fulcher et al., 1984). Moreover, plasminogen activator inhibitor 1 is neutralized by activated protein C and fibrinolytic activity is increased (Sakata et al., 1985). On the other hand, thrombomodulin-bound thrombin inhibits fibrinolysis of the newly formed clot by activation of thrombin-activatable fibrinolysis inhibitor (TAFI). Activated TAFI has additionally several anti-inflammatory properties by inactivating the complement peptides C3a and C5a (Campbell et al., 2002) and the inflammatory mediator and vasodilator bradykinin (Shinohara et al., 1994). Petersen et al., (2009) generated triple transgenic pigs by adding a CMV-driven human thrombomodulin gene to CD55, CD59 transgenic animals and observed thrombomodulin expression in all organs. 
In vitro human thrombomodulin coactivity assay with porcine fibroblasts showed increased activated protein $\mathrm{C}$ production, but the porcine coagulation system was not perturbed, allowing pigs to be raised normally. Transgenic pigs, based on a GGTA1 knockout/CD46 background, expressing human thrombomodulin on endothelial cells have been produced by Wuensch et al., (2012). Pigs were screened for loss of the $\alpha$ Gal-epitopes and CD46/ human thrombomodulin expression. Several triple transgenic animals were generated and used for pig-to-baboon heterotopic/orthotopic heart transplantation to verify the positive effects of human thrombomodulin for xenotransplantation. In a heterotopic transplantation model hearts from GGTA1 knockout/CD46 animals survived between of 21 to 80 days, whereas hearts from GGTA1 knockout/CD46/human thrombomodulin animals survived on average for more than 200 days, indeed some animals surpassed one (Mohiuddin et al., 2014) and even two years (MIT Report, 2015).

\subsection{Endothelium protective genes}

The different strategies mentioned here are primarily directed at inhibiting the hyperacute rejection and activation of the coagulation system. Longer term xenograft survival also requires protection of the graft endothelial cells against inflammation and apoptosis. This can be achieved by overexpressing antiapoptotic and anti-inflammatory genes.

\subsubsection{Heme oxygenase 1}

As mentioned previously, $\alpha$ Gal-deficient porcine xenografts are eventually rejected and commonly exhibit thrombotic microangiopathy. The probable cause is continual activation of the xenograft endothelium by anti-pig antibodies (Pierson 2009). Proteins with vascular protective properties, such as heme oxygenase 1 (HO1) or tumor necrosis factor $\alpha$ induced protein 3 (A20) are able to inhibit this process.

Heme oxygenase has three different isoforms. HO2 is constitutively active whereas HO3 is non-catalytic and functions as an oxygen sensor. 
H01 is ubiquitously expressed at low levels in all tissues and is induced by high heme concentrations. It is highly expressed in spleen where it regulates the levels of free iron from senescent erythrocytes (Ferris et al., 1999).

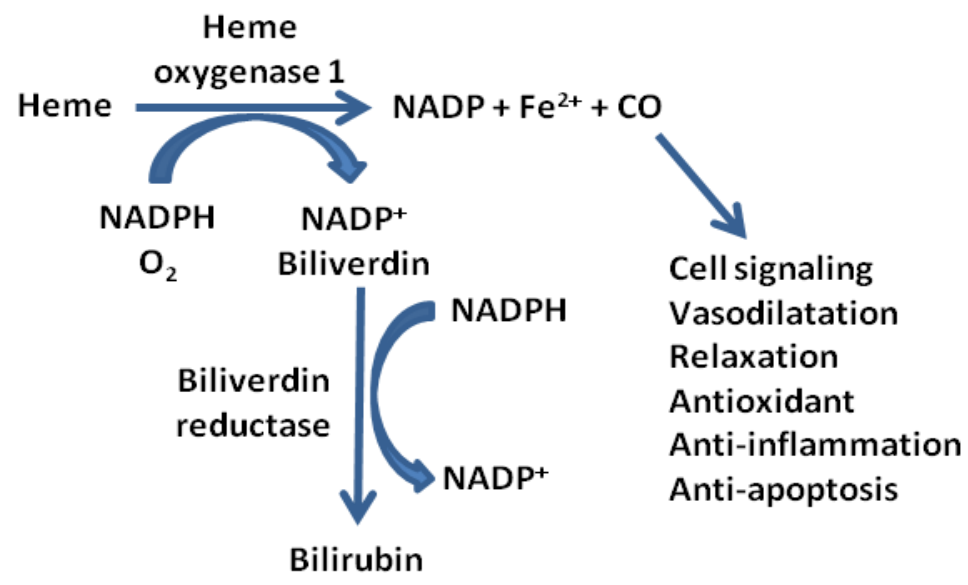

Figure 5. Biological functions of HO1 and graft protective properties.

HO1 degrades free heme to biliverdin, free iron and carbon monoxide (CO), which has strong anti-apoptotic and anti-inflammatory effects (Fig. 5). Biliverdin is degraded by biliverdin reductase to bilirubin. Free iron upregulates the expression of heavy chain (H-) ferritin which activates an ATPase dependent iron pump and reduces intracellular iron levels (Eisenstein et al., 1991).

HO1 also has several immunomodulatory functions. It reduces the activity of NK cells and the formation of pro-inflammatory factors such as the chemokine receptor type 5 (CCR5) and the intercellular adhesion molecule 1 (ICAM-1). Overexpression of H01 has been shown to have strong anti-inflammatory effects, reducing thrombus formation and IgM deposition on xenografts (Wang et al., 2002; Zhen-Wei et al., 2007). Other properties of HO1 important for xenotransplantation are inhibition of platelet aggregation, maintenance of microcirculation and facilitation of angiogenesis (Bhang et al., 2011; Katori et al., 2002). Prolonged xenograft survival of rodent heart and lung xenografts confirmed the protective effects of $\mathrm{HO1}$ for xenotransplantation (Sato et al., 2001; Tabata et al., 2003). Several groups have produced HO1 transgenic pigs. 
Petersen et al. (2011) generated transgenic pigs with SV40 promoter driven HO1 cDNA and observed HO1 expression in heart, kidney, liver, endothelial cells and fibroblasts.

Aortic endothelial cells from these animals were protected from TNF- $\alpha$-mediated apoptosis and kidneys could be perfused ex vivo with human AB-pooled blood for 240 minutes compared to 60 minutes with wild-type (Petersen et al., 2011). Yeom et al. (2012) reported transgenic pigs with CMV-driven HO1 cDNA and observed expression in almost all tissues including the heart, kidney, lung, pancreas, spleen and skin. Fibroblasts were resistant to hydrogen peroxide damage, as well as TNF- $\alpha$ and cycloheximide-mediated apoptosis.

\subsubsection{A20}

Expression of the TNFAIP3 (tumor necrosis factor alpha induced protein 3,A20) gene is induced by the nuclear factor kappa-light-chain-enhancer of activated Bcells (NF- $\mathrm{KB}$ ). A20 encodes a zinc-finger protein that acts as a K48-ubiquitin ligase and K63-deubiquitinase and is involved in cytokine-mediated immune and inflammatory responses. A20 uses a negative feedback loop to inhibit NF-кB activation and tumor necrosis factor (TNF)-induced apoptosis (Krikos et al., 1992). Inactivation of NF- $\kappa B$ is essential for xenotransplantation because NF- $\kappa B$ transcription factors upregulate pro-inflammatory cytokines including the tumor

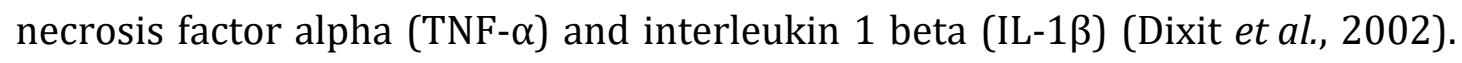
A20-deficient mice are unable to downregulate NF- $\kappa B$ activation resulting in chronic inflammation and cell death (Lee at al., 2000). NF- $\kappa \mathrm{B}$ is normally sequestered in the cytoplasm as complexes with IкB proteins (Fig. 6). Activation of the TNF-receptor activates a series of factors including the receptor interacting protein (RIP), TNF-receptor-associated factor (TRAF), various NF- $\kappa B$ essential-modifier proteins and the IKB kinase $\gamma(\mathrm{IKK} \gamma)$, which recruit I $\mathrm{I} B$ kinases leading to the phosphorylation, ubiquitination and degradation of $\mathrm{I} \kappa \mathrm{B}$. $\mathrm{NF}-\kappa \mathrm{B}$ translocates to the nucleus and activates inflammatory responses (Karin et al., 2000). A20 interferes with this process at several stages. The two ubiquitin-editing domains of A20 inhibit NF- $\mathrm{B}$ signalling. 
The N-terminal domain of A20 functions as a deubiquitinase and removes lysine63 linked ubiquitin chains from the receptor interacting protein (RIP) that are essential for TNF receptor signalling (Ting et al., 1996). The C-terminal domain of A20 consists of seven zinc finger domains and acts as an ubiquitin ligase which poly-ubiquitinylates lysine-48 of RIP, marking it for degradation by the proteosome (Fig. 6) (Opipari et al., 1990).

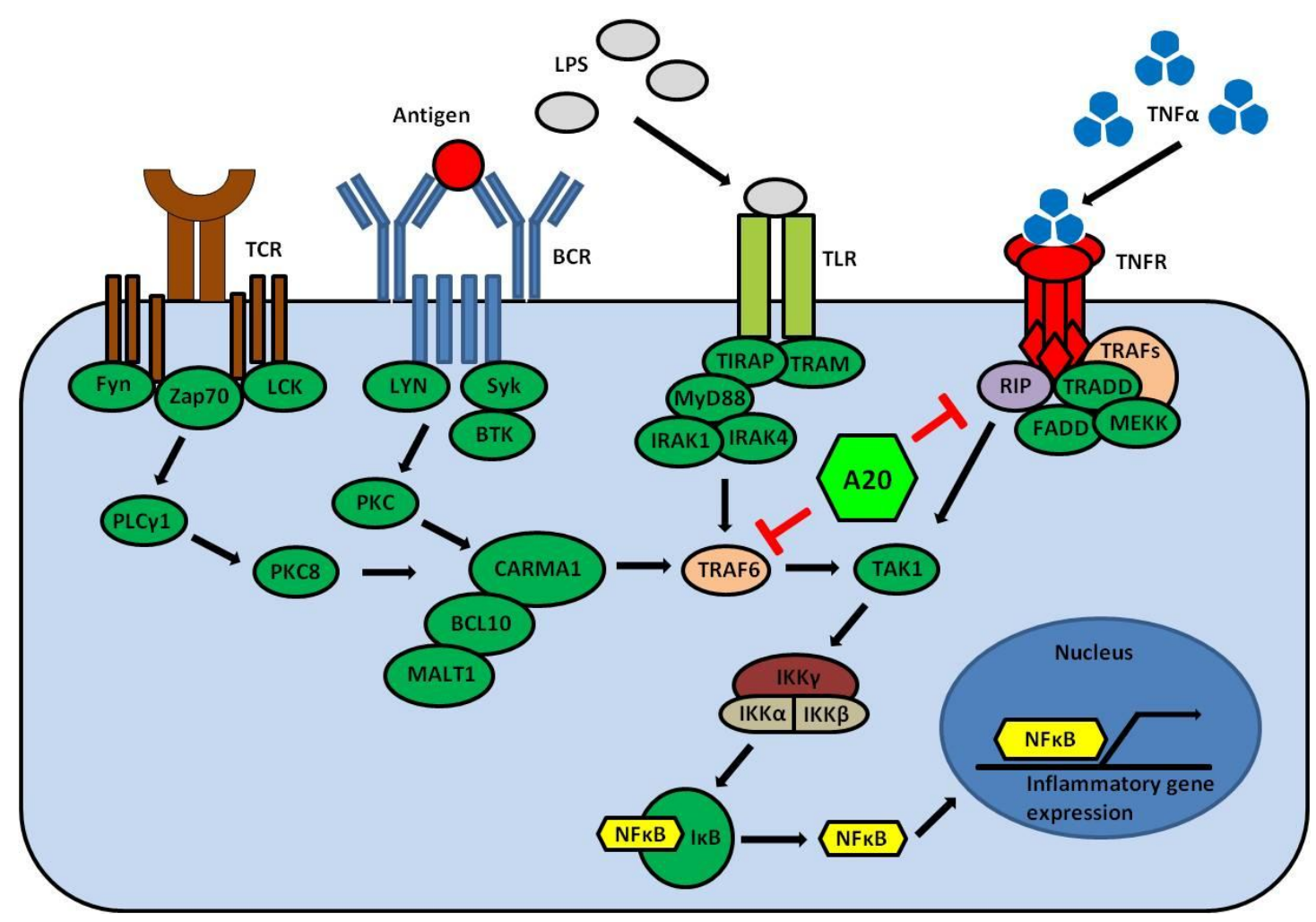

Figure 6. Functions of A20. A20 inhibits activation of NF-KB by its K48-ubiquitin ligase and K63deubiquitinase domains by blocking the signaling pathways of factors including the receptor interacting protein (RIP) and the TNF-receptor-associated factor (TRAF) (adapted from www.sinobiological.com/TNFAIP3-protein-wm03719.html)

$N F-\kappa B$ signaling can also be initiated by the $\mathrm{T}$ cell receptor (TCR)/CD28 interaction. The Carma1-Bcl10-Malt1 complex is responsible for the interaction of the TCR/CD28 complex and the NF- $\mathrm{kB}$ signaling cascade. By removing K63 linked ubiquitin chains from Malt1, A20 prevents interaction and inhibits NF- $\kappa B$ activation. A20 itself can be degraded by the para-caspase activity of Malt1,

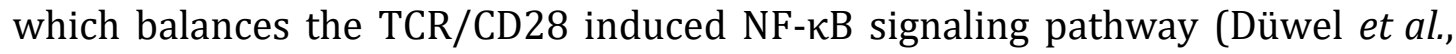
2009). As Malt1 is indispensable for T cell activation and proliferation, A20 also suppresses T cell activation (Coornaert et al., 2008). 
Oropeza et al., (2009) produced transgenic pigs with A20 cDNA expression directed by the synthetic CAG (CMV enhancer/chicken $\beta$-actin/rabbit globin) promoter and detected A20 in heart, muscle and porcine aortic endothelial cells (PAEC). PAECs were resistant to TNF $\alpha$ induced apoptosis. After ischemia/reperfusion injury, infarct size was similar to the non-transgenic control, but A20 transgenic pig hearts showed significantly lower myeloperoxidase activity and better hemodynamic performance, suggesting partial protection of cardiomyocytes.

\subsection{Immune regulatory genes and cell mediated xenograft rejection}

The next hurdle for xenograft survival is cell-mediated rejection caused by $\mathrm{T}$ cells, natural killer (NK) cells and macrophages.

Porcine cells express several stimulatory ligands that cause activation of T cells, NK cells and macrophages (Fig. 7). Immunosuppressive drugs can avoid a cellular response, but drugs do not provide full protection, they can be toxic and long-term systemic immunosuppression can cause many deleterious effects (McGregor et al., 2005).

\subsubsection{NK cells}

Human xenoreactive antibodies that recognize porcine antigens can activate NK cells and macrophages through interaction with the Fc receptor for IgG (Fc $\gamma \mathrm{R})$. NK cells can be activated by interaction of a NK-cell receptor such as the naturalkiller group 2 member D (NKG2D) with the UL16-binding protein 1 (ULBP1) which is expressed on porcine cells (Lilienfeld et al., 2006). A so far unknown ligand on porcine cells can also activate NK cells through interaction with the NK-cell receptor NKp44 (Forte et al., 2005). Some inhibitory NK cell receptors, for example killer-cell lectin-like receptor subfamily G member 1 (KLRG1), recognize porcine ligands such as E-cadherin (Ito et al., 2006) but porcine cells cannot protect themselves from NK cell-mediated cell lysis because the porcine major histocompatibility complex (SLA) class I is not able to interact with the inhibitory NK cell receptor (Seebach et al., 1997). 


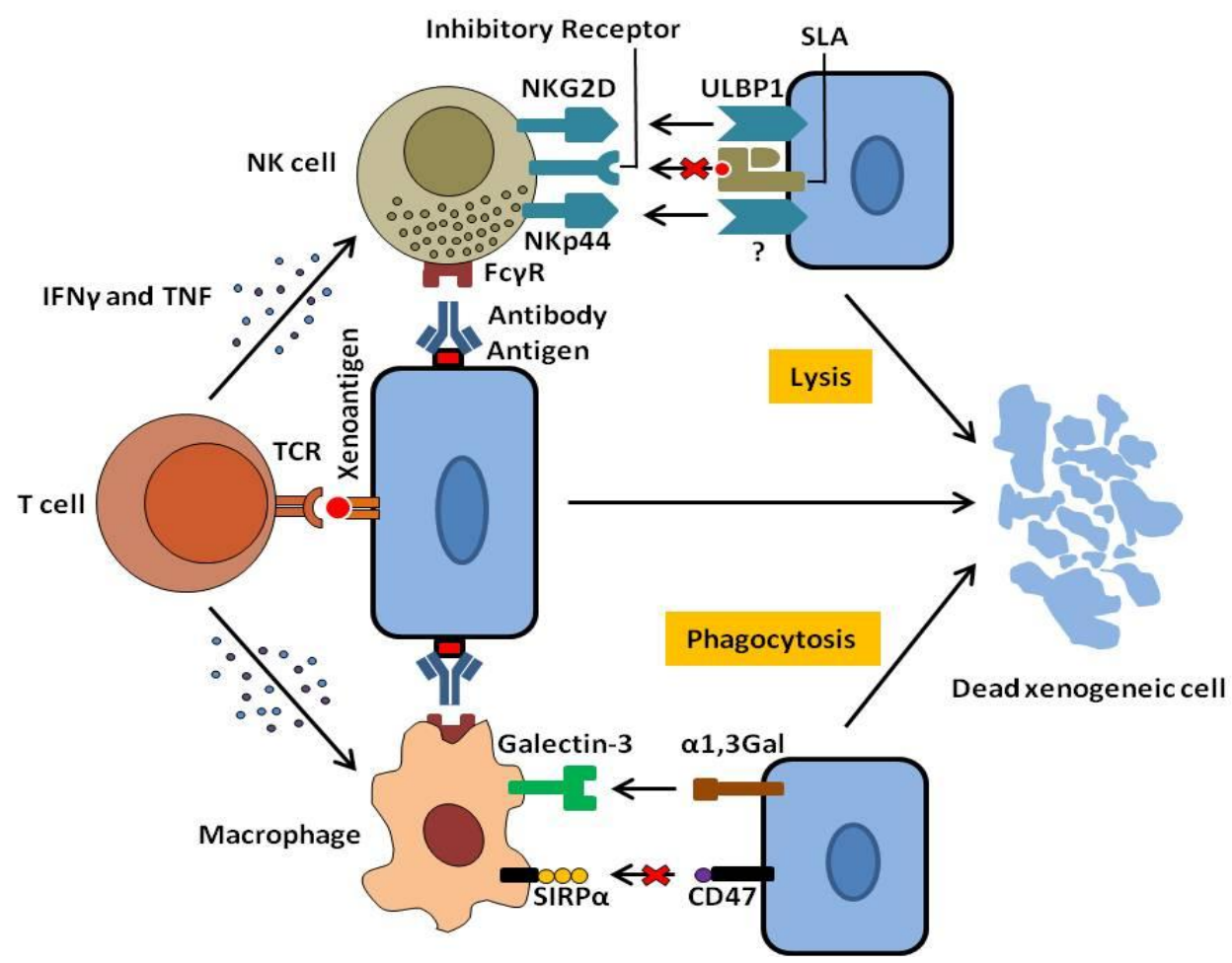

Figure 7. Cellular rejection mechanisms caused by xenogeneic cells after transplantation (adapted from Yang and Sykes, 2007).

\subsubsection{Macrophages}

Macrophages can be activated by the interaction of porcine $\alpha$ Gal epitopes with the macrophage receptor galectin-3 (Jin et al., 2006). As the porcine inhibitory ligand CD47 does not interact with the signal-regulatory protein- $\alpha$ (SIRP $\alpha$ ) on human macrophages, porcine cells are not protected against macrophage phagocytosis (Wang et al., 2007; Ide et al., 2007). Cytokines including IFN $\gamma$ or TNF, produced by activated T cells, can significantly increase the cytotoxicity of NK cells and macrophages (Yi et al., 2003). 


\subsubsection{T-cells}

T cell-mediated xenograft rejection is clearly important for xenotransplantation of solid porcine organs and porcine pancreatic islet cells. T cell activation by xenografts is initiated by the presentation of xenograft-derived peptides by the major histocompatibility complex (MHC) of host antigen presenting cells (APCs) to host $\mathrm{CD}^{+} \mathrm{T}$ cells. Complete $\mathrm{T}$ cell activation usually depends on costimulatory signals between CD80/86 (B7 ligands on APCs) and CD28 (on T cells) or CD40 and CD40L (CD154). In contrast to human CD8+ $\mathrm{T}$ cells, human $\mathrm{CD}^{+} \mathrm{T}$ cells can recognize porcine MHC (SLA) class I (Yamada et al., 1995; Dorling et al., 1996) and, because porcine endothelial cells constitutively express CD80/86, xenografts can activate human T cell responses (Murray et al., 1994). The helper function of $\mathrm{CD} 4^{+} \mathrm{T}$ cells is required for $\mathrm{CD}^{+} \mathrm{T}$ cells to proliferate (Dorling et al., 1996).

Blocking of the co-stimulatory pathway between CD80/86-CD28 by cytotoxic T lymphocyte-associated antigen 4 (CTLA4) or CD40-CD40L by a CD40L monoclonal antibody prolongs survival of rodent xenografts (Lenschow et al., 1992; Gordon et al., 2001). CTLA4 binds CD80/86 with higher affinity than CD28 and downregulates T cell activation (Linsley et al., 1991; Linsley et al., 1994). Transgenic pigs expressing CAG promoter driven porcine CTLA4 fused to the hinge and $\mathrm{CH} 2 / \mathrm{CH} 3$ regions of human IgG1 (pCTLA4-Ig) have been produced by Phelps et al. (2009). These pigs showed pCTLA4-Ig expression in all solid organs examined and in blood. However, these animals were very susceptible to infections and required frequent antibiotic treatment. Klymiuk et al. (2012) reported pancreatic $\beta$-cell-specific expression of an improved high-affinity derivative LEA29Y directed by the porcine insulin promoter. Transgenic pigs were healthy and showed strong $\beta$-cell-specific transgene expression. 


\subsection{BAC and PAC vectors}

As this account indicates, effective protection of xenografts requires a huge range of graft-protective genes to inhibit the various rejection mechanisms. The remaining sections of this introduction focus on means of achieving expression of multiple xenoprotective genes in transgenic animals.

Most transgenic animals so far generated for xenotransplantation contain either cDNA or minigene constructs. These smaller constructs are easy to generate and to manipulate and also allow gene targeting approaches, but as explained later, are often silenced by the local chromatin environment. cDNA based constructs also do not support the expression of alternate RNA splicing variants and may thus lack important protein isoforms. The use of large genomic constructs can solve both these problems. Molecular manipulations are however more difficult, but useful technologies have been established to enable the construction, analysis and transfer of large genomic transgenes. Bacterial artificial chromosomes (BAC) and phage artificial chromosomes (PAC) were developed to carry inserts of several hundred kilobases and so enable molecular cloning of a complete genomic locus including all regulatory elements responsible for controlling gene expression. BAC vectors have been used to clone DNA fragments from $300 \mathrm{~kb}$ up to $750 \mathrm{~kb}$ (Shizuya et al., 1992; Stone et al., 1996) and PAC vectors are capable of carrying inserts between 100-300 kb (Coren and Sternberg, 2001). The initial development of BAC and PAC libraries was largely driven by the Human Genome Project to allow construction of genomic libraries and physical maps for genome sequence analysis.

The BAC system is based on the single-copy Escherichia coli F-factor plasmid and the PAC system is derived from the P1 bacteriophage. Both provide facile cloning and structural stability of cloned DNA fragments. BACs are present at low copy number in bacterial cells, which enables stable maintenance of large DNA fragments and reduces the probability of recombination between DNA fragments.

The position at which a transgene integrates into the host genome has a powerful effect on expression. 
The use of large genomic transgenes has a major advantage over smaller constructs because they are more likely to contain chromatin organizing domains, making them less susceptible to position effects and long-term gene silencing by the local chromatin environment (Shizuya, Kouros-Mehr, 2001).

\subsection{Human artificial chromosomes and MMCT}

Artificial chromosomes are another type of vector capable of carrying large genomic constructs. In principle these have several advantages that led to their investigation as means of delivering large tracts of DNA. Because they are completely separate chromosomes that replicate and segregate like normal chromosomes they avoid any integration into the host genome and so avoid any risk of insertional mutagenesis and disruption of endogenous genes. They should also be free of copy number variation and variations in expression due to the local chromatin environment, the position effect. Human artificial chromosomes (HAC) have been developed that can carry up to $10 \mathrm{Mb}$ of genomic DNA. The first linear HACs were made by cotransfection of alpha-satellite DNA, telomeric DNA and genomic DNA into human HT1080 cells. This resulted in a completely new microchromosome formed from these components, and which exhibited a high level of structural and mitotic stability (Harrington et al., 1997). HAC formation de novo using a circular PAC vector containing $70 \mathrm{~kb}$ of alpha-satellite DNA revealed that alpha-satellite DNA is important for HAC formation and that telomere sequences are dispensable in circular HACs (Ebersole et al., 2000), also that non-alphoid DNA can act as a neocentromere and support artificial chromosome formation (Amor et al., 2004, Saffery and Choo, 2002). Further research on HAC formation de novo has however encountered several problems such as failure of HAC formation and integration of the HAC components into the host genome, and has only been successful in cells with high telomerase activity e.g. human HT1080 or HeLa cells. Work in this area has therefore largely shifted to truncation and modification of natural chromosomes, for example by homologous recombination of telomeric repeats into natural chromosomes and subsequent telomere-directed fragmentation, or radiation-induced breakage. 
Most experiments have been conducted in human cells or human-hamster hybrid cell lines. However, the low efficiency for homologous recombination in these cells made chicken DT40 cells with a high level of homologous recombination the preferred cell line. (Kuroiwa et al., 1998; Kazuki et al., 2011; Kakeda et al., 2011; Dieken et al., 1996).

After successful HAC formation, parameters such as chromosomal stability, the arrangement of whole chromosomal loci, gene regulation mechanisms and expression patterns can further be analyzed. Therefore, the modified chromosomes have to be transferred from one cell line to another to perform the analysis in a different genetic background.

Microcell-mediated chromosome transfer (MMCT) enables transfer of exogenous chromosomes or truncated chromosomes between different cell lines. Briefly, a donor cell line with a selectable marker is induced to form micronuclei by exposure to colcemid, which inhibits formation of the mitotic spindle and blocks the cell cycle in metaphase. If the exposure time is long, some cells pass through this block and enter an aberrant $\mathrm{G}_{1}$ stage in which each chromosome is encased with its own nuclear membrane. These multinucleated cells can be induced to form microcells by treatment with cytochalasin B and high speed centrifugation (20000-40000g). These microcells can then be fused using a fusogenic reagent such as polyethylene glycol with the recipient cell line, which is subsequently selected (Ege and Ringertz, 1974). MMCT has been used for many decades in several fields to identify tumor suppressor and senescence-linked genes, to study genomic imprinting, DNA repair mechanisms, suppression of telomerase activity in cancer cells and to generate transchromosomal animals (Meaburn et al., 2005). Although intra-species chromosome transfer works efficiently, interspecies chromosome transfer faces several problems. As a de novo HAC has to be made in a permissive cell type, e.g. human HT1080 cells, it is necessary to transfer the HAC into the cell type of interest. Transfer efficiency is however very low, ranging from $10^{-4}$ to $10^{-6}$ in successful experiments (Katoh et al., 2004; Kakeda et al., 2005). After MMCT, HACs often undergo chromosomal rearrangements depending on the genetic background, the centromeric sequence and the telomere length. 
In humans, the centromeric sequence consists predominantly of tandemly repeated $171 \mathrm{bp}$ alpha-satellite DNA monomers. These monomers form higherorder repeats, of which each contains a specific number of monomer units. The specific subfamiliy of alpha-satellite DNA seems to influence the genetic stability of HACs in the recipient cell line (Choo et al., 1991). MMCT has been used to transfer HACs into cattle (Kuroiwa et al., 2002) and mice (Hernandez et al., 1999; Tomizuka et al., 1997). However, a HAC transfer is labor intensive and time consuming (Ikeno et al. 2002) but several groups produced transgenic mice and could show germline transmission and structural stability of linear and circular HACs (Suzuki et al., 2006; Tomizuka et al., 1997).

\subsection{Genetic modifications in livestock}

Genetically modified animals were and are created either by random transgene integration or by targeted modifications using embryonic stem (ES) cells or somatic cells. As fully functional ES cells are not available for most livestock species, the development of somatic cell nuclear transfer (SCNT) is additionally presented.

\subsubsection{Transgenic livestock}

Since the 1980s the availability of mouse ES cells has enabled genetic modifications of endogenous genes, e.g. insertions, deletions and substitutions, by gene targeting via homologous recombination and transfer of the modified genotype to whole animals. This incredibly powerful technology is made possible by three key features of ES cells: they can be cultured as pluripotent cells for long periods in culture while maintaining a normal karyotype (Evans and Kaufmann, 1981); they support homologous recombination at high frequency, far greater than somatic cells (Sedivy et al. 1999); and they can contribute to the formation of chimeric mice via incorporation into early embryos (Bradley et al., 1984). Despite considerable efforts it has however not been possible to derive fully functional ES cells for livestock species. This motivated investigation of SCNT as an alternative. 
Nuclear transfer in mammals was originally achieved with embryonic blastomeres (Willadsen 1986) and for a decade could not be extended to any other cell type (McGrath and Solter, 1984) but an understanding of the importance of the cell cycle stage of the donor cell allowed SCNT using embryonic cells cultured for extended periods (Campbell et al., 1996). This was later extended to cultured adult somatic cells (Wilmut et al., 1997) and the production of the first gene targeted animals (McCreath et al., 2000).

\subsubsection{Precise genetic modifications}

The general structure of a vector designed to carry out gene targeting by homologous recombination (HR), is two DNA fragments homologous to the chromosomal target region flanking the desired alteration and some form of selectable marker. This is introduced into the cell where it gains access to the nucleus, usually after breakdown of the nuclear membrane, and recombines with the endogenous target sequence. Cells that have undergone double crossover events leading to incorporation of the desired alteration are then selected e.g. by drug resistance. The position of the homology arms determines where the sitespecific modifications take place. Several gene-targeting approaches via HR in sheep, cattle and pig (McCreath et al., 2000; Denning et al., 2001; Sendai et al., 2003; Rogers et al., 2008) verified the method of HR itself for genetic modifications in somatic cells but also revealed problems. In mouse ES cells the optimal total length of homologous sequences was determined as between 5 and $8 \mathrm{~kb}$ with a minimum size of $0.5 \mathrm{~kb}$ per homology arm (Hasty et al., 1991; Hasty et al., 1993). For targeting approaches in somatic cells the systematic analysis of homology sequence sizes is rarely possible due to the very low targeting efficiencies. The total length of homologous regions used was between 6.4 and $11.3 \mathrm{~kb}$ (Sendai et al., 2006; Kuroiwa et al., 2004; Denning et al., 2001) with the shortest homology arm of $0.75 \mathrm{~kb}$ (Jin et al., 2003). For recombinant adenoassociated viruses, whose size limit is about $5 \mathrm{~kb}$, shorter homology sequences of total 2.2 and $2.4 \mathrm{~kb}$ were used (Rogers et al., 2008; Sun et al., 2008).

Several methods have been established to deliver gene targeting vectors or plasmids into cells. 
DNA microinjection achieves only low efficiencies in somatic cells and requires special equipment and expertise. Chemical methods based on liposomes, electroporation and viral vectors are the methods of choice for somatic cells. For each cell type, the best transfection method has to be determined separately. To increase targeting efficiencies, the addition of a nuclear localization sequence (NLS) to the targeting vector and cell synchronization in S-phase can be beneficial (Mir et al., 2004). Adeno-associated viruses can transduce many different cell types and achieve high efficiencies of up to 1\% for HR (Russell et al., 1998). However, low targeting efficiencies remain a general problem and improved methods such as promoter-trap, poly-A-trap or negative selection markers were developed to further enhance site-specific gene-targeting. These gene-trap approaches consist either of a promoterless gene or a gene without poly-A signal. If homologous recombination takes place, the gene is inserted either in front of an active promoter or a functional poly-A site. In case of random integration, the gene is not transcribed. If a negative selection marker is used, a negative selection cassette, which is located outside of the homology arms, is additionally part of the targeting vector. In case of homologous recombination the negative selection cassette is not inserted in the chromosomal DNA. If a random integration of the targeting vector takes place, the negative selection cassette is integrated and cells die after selection.

Despite all improvements, gene modifications using HR are however still very inefficient and often need many attempts for a successful targeting. Gene knockouts using HR depend on the insertion of a selection cassette and the frequency is very low $\left(10^{-6}-10^{-5}\right)$ (Denning et al., 2001; Jin et al., 2003). However, double stand breaks at the target site can considerably improve the frequency of homologous recombination (Taghian and Nickoloff 1997; Elliott and Jasin 2001). Due to the low efficiency of HR, new techniques were developed. Zinc-finger nucleases (ZFNs), transcription activator-like effector nucleases (TALENs) or clustered regularly interspaced short palindromic repeats (CRISPRs) allow precise genetic modifications with much higher efficiencies.

ZFNs are dimeric structures consisting of a pair of DNA-binding domains fused to FokI endonuclease domains. When a pair of ZFNs bind to their target motifs, the FokI domains can dimerize and cause a double-strand break (Kim et al., 1996). 
These double-strand breaks can be repaired by homologous recombination or by non-homologous end joining (NHEJ) which causes small insertions or deletions (Porteus et al., 2005) and finally results in a non-functional protein. ZFNs have been used to generate gene disruptions in animals including fruit fly (Bibikova et al., 2002), mice (Carbery et al., 2010; Meyer et al., 2010), rats (Cui et al., 2011), rabbits (Flisikowska et al., 2011) and pigs (Hauschild et al., 2011). However, the design and production of high quality specific ZFNs requires considerable time and labor-intensive optimization, whereas TALENs or CRISPRs are very easy to design and to produce by modular assembly.

TALENs dimers consist of a DNA-binding motif which like ZFNs are fused to FokI nuclease domains. The DNA target sequence is recognized by repeat domains that contain variable two amino acid pairs that determine their specificity (Cermak et al., 2011). One pair of TALENs binds to their target sequences and causes dimerization and activation of the FokI nuclease which results in a double-strand break that can be repaired by HR or NHEJ. TALENs have achieved targeting efficiencies between 2\% and 55\% (Miller et al., 2011; Reyon et al., 2012) and were used for the targeting of a large number of genes in livestock (Carlson et al., 2012).

The CRISPR type II system is a part of the adaptive immune system of bacteria and archaea to protect themselves against viruses and phages. The CRISPR RNA (crRNA) and the trans-activating crRNA (tracrRNA) form a two-RNA structure to guide Cas9 nuclease to its target DNA sequence where a double-strand break is caused. For efficient genetic manipulation of cells, the 3' end of crRNA was fused to the $5^{\prime}$ end of tracrRNA to produce a single chimeric RNA molecule (Jinek et al., 2013). The CRISPR/Cas9 system has been used for a large amount of gene knockouts in plants e.g. arabidopsis, tobacco, rice and soghum (Jiang et al., 2013) as well as in bacteria, yeast, fruit fly, zebrafish, human cells and livestock animal cells (Mali et al., 2013). 


\subsection{Porcine ROSA26}

As mentioned earlier, the site at which an exogenous transgene integrates can have a powerful influence on its expression. Therefore, using gene targeting to place transgenes at a particular predetermined locus in the genome can be advantageous.

In mice the Rosa26 locus is widely used to place transgenes because it is broadly permissive for transgene expression. The murine Rosa26 promoter was identified by random retroviral gene trapping in embryonic stem cells (Friedrich et al., 1991) and promotes moderate expression levels. The murine Rosa26 locus encodes two non-coding transcripts and one evolutionarily highly conserved transcript, the function of which is unknown (Zambrowicz et al., 1997). Targeting of this locus using a $\beta$-gal reporter construct revealed ubiquitous expression in mouse embryos and hematopoietic cells and had no effects on embryonic development or fertility (Zambrowicz et al., 1997). The human and rat ROSA26 loci were subsequently identified, used for targeted transgene placement and found to support ubiquitous expression (Irion et al., 2007; Kobayashi et al., 2012). Other members of the Chair of Livestock Biotechnology have identified and characterized the porcine ROSA26 locus (Li et al., 2014) and demonstrated that as in other species it supports ubiquitous transgene expression. These properties of the porcine ROSA26 locus are useful for xenotransplantation. Targeted placement at the ROSA26 locus offers a useful means of achieving high and ubiquitous expression of xenoprotective transgenes. 


\subsection{Project goals}

The aim of this work was to create multi-transgenic donor animals that inhibit the various steps of xenograft rejection. This requires levels of transgene expression sufficient to protect the graft, and to combine the different xenogenes in one animal at one single genomic locus.

Expression constructs. Two types of expression constructs were to be investigated - BAC/ PAC constructs and minigenes. The original size of the BAC/PAC constructs was to be reduced to place additional xenograft-protective genes on these constructs. This could enable easier handling and would reduce the risk of multiple genomic integration sites. Several minigenes should be prepared, suitable for targeted transgene placement. Moreover, different types of promoters (CAG, SV40, gene-specific) should be examined to obtain high expression levels.

Subsequently, two different strategies were to be used to obtain multi-transgenic animals.

Cotransfection experiments. The large BAC and PAC vectors should be used in cotransfection experiments to obtain either HACs carrying multiple xenoprotective transgenes or to obtain cell clones expressing a large number of transgenes at once. Cell clones with high expression levels of the complement regulatory genes were to be used for SCNT to create transgenic animals.

Transgene placement at the porcine ROSA26 locus. The porcine ROSA26 locus should be targeted by a cDNA construct of heme oxygenase 1 . Correct targeted clones were to be used for SCNT to obtain a transgenic animal. A gene-stacking approach using a CAG-CD55 minigene should be prepared. 


\section{Materials and Methods}

\subsection{Chemicals}

\begin{tabular}{|c|c|}
\hline Acetic acid & $\begin{array}{l}\text { Fluka Laborchemikalien GmbH, Seelze, } \\
\text { Germany }\end{array}$ \\
\hline Bovine serum albumin (BSA) & New England Biolabs, Frankfurt, Germany \\
\hline $\begin{array}{l}\text { 5-Bromo-4-chloro-3-inolyl- } \beta \text {-D- } \\
\text { galactopyranosid }\end{array}$ & $\begin{array}{l}\text { Sigma-Aldrich Chemie GmbH, Steinheim, } \\
\text { Germany }\end{array}$ \\
\hline Bromphenol blue & $\begin{array}{l}\text { Sigma-Aldrich Chemie GmbH, Steinheim, } \\
\text { Germany }\end{array}$ \\
\hline Calcium Chloride & Merck KGaA, Darmstadt, Germany \\
\hline $\begin{array}{l}\text { 4',6'-Diamidino-2'-phenylindole- } \\
\text { dihydrochloride (DAPI) }\end{array}$ & $\begin{array}{l}\text { Roche Diagnostic GmbH, Mannheim, } \\
\text { Germany }\end{array}$ \\
\hline Dimethyl sulfoxide (DMSO) & $\begin{array}{l}\text { Sigma-Aldrich Chemie GmbH, Steinheim, } \\
\text { Germany }\end{array}$ \\
\hline Dithiotreitol (DTT) & $\begin{array}{l}\text { Omnilab Life Science OLS, Bremen, } \\
\text { Germany }\end{array}$ \\
\hline Ethanol 96\% & Riedel-de-Haen, Seelze, Germany \\
\hline Ethidium bromide $(10 \mathrm{mg} / \mathrm{ml})$ & $\begin{array}{l}\text { Sigma-Aldrich Chemie GmbH, Steinheim, } \\
\text { Germany }\end{array}$ \\
\hline Ethylenediamintetraaceticacid (EDTA) & $\begin{array}{l}\text { Sigma-Aldrich Chemie GmbH, Steinheim, } \\
\text { Germany }\end{array}$ \\
\hline Formalin $10 \%$ & $\begin{array}{l}\text { Sigma-Aldrich Chemie GmbH, Steinheim, } \\
\text { Germany }\end{array}$ \\
\hline Formamide & $\begin{array}{l}\text { Sigma-Aldrich Chemie GmbH, Steinheim, } \\
\text { Germany }\end{array}$ \\
\hline GenAgarose L.E. & $\begin{array}{l}\text { Genaxxon Bioscience GmbH, Biberach, } \\
\text { Germany }\end{array}$ \\
\hline Glycerol 99\% & Carl Roth GmbH, Karlsruhe, Germany \\
\hline Glycine & Carl Roth GmbH, Karlsruhe, Germany \\
\hline Heparin disodium salt & $\begin{array}{l}\text { Sigma-Aldrich Chemie GmbH, Steinheim, } \\
\text { Germany }\end{array}$ \\
\hline Human Serum & Dunn Labortechnik, Germany \\
\hline Interferon gamma (porcine) & $\mathrm{R}+\mathrm{D}$ systems \\
\hline Isolectin B4, biotin-conjugated & Enzo Life Sciences, Lörrach, Germany \\
\hline Isolectin B4-FITC & Enzo Life Sciences, Lörrach, Germany \\
\hline
\end{tabular}




\begin{tabular}{ll}
\hline Isopropanol & Carl Roth GmbH, Karlsruhe, Germany \\
\hline Kanamycin (Kana) & $\begin{array}{l}\text { Sigma-Aldrich Chemie GmbH, Steinheim, } \\
\text { Germany }\end{array}$ \\
\hline Loading dye (5×) & $\begin{array}{l}\text { Sigma-Aldrich Chemie GmbH, Steinheim, } \\
\text { Germany }\end{array}$ \\
\hline -Mercaptoethanol & $\begin{array}{l}\text { Sigma-Aldrich Chemie GmbH, Steinheim, } \\
\text { Germany }\end{array}$ \\
\hline Methanol & $\begin{array}{l}\text { Sigma-Aldrich Chemie GmbH, Steinheim, } \\
\text { Germany }\end{array}$ \\
\hline Potassium acetate & Riedel-de Haen GmbH, Seelze, Germany \\
\hline Sodium-51-chromate & GE Healthcare, Buckinghamshire, UK \\
\hline Sodium dodecyl sulfate (SDS) & $\begin{array}{l}\text { Sigma-Aldrich Chemie GmbH, Steinheim, } \\
\text { Germany }\end{array}$ \\
\hline Sodium acetate & Roth, Karlsruhe, Germany \\
\hline Sodium hydroxide & Riedel-de Haen GmbH, Seelze, Germany \\
\hline Sodium chloride & $\begin{array}{l}\text { Sigma-Aldrich Chemie GmbH, Steinheim, } \\
\text { Germany }\end{array}$ \\
\hline Sodium pyruvate & PAA, Pasching, Austria \\
\hline TNF alpha (human) & Biomol, Hamburg, Germany \\
\hline Trizima base (Tris base) & Sigma-Aldrich Chemie GmbH, Steinheim, \\
& Germany \\
\hline Trizol & Invitrogen, Karlsruhe, Germany \\
\hline Tris hydrochloid (Tris HCl) & Sigma-Aldrich Chemie GmbH, Steinheim, \\
Trypan blue solution $(0.4 \%)$ & $\begin{array}{l}\text { Sigma-Aldrich Chemie GmbH, Steinheim, } \\
\text { Germany }\end{array}$ \\
\hline Tween 20 & $\begin{array}{l}\text { Sigma-Aldrich Chemie GmbH, Steinheim, } \\
\text { Germany }\end{array}$ \\
\hline & \\
\hline
\end{tabular}

\subsection{Enzymes}

\begin{tabular}{ll}
\hline CIP & New England Biolabs, Frankfurt, Germany \\
\hline DNA Polymerase I, Large Klenow Fragment & New England Biolabs, Frankfurt, Germany \\
\hline GoTaq DNA Polymerase & Promega GmbH, Mannheim, Germany \\
\hline PCR Extender System & 5Prime GmbH, Hamburg, Germany \\
\hline Proteinase K & $\begin{array}{l}\text { Sigma-Aldrich Chemie GmbH, Steinheim, } \\
\text { Germany }\end{array}$ \\
\hline Q5 Hot Start High-Fidelity DNA Polymerase & New England Biolabs, Frankfurt, Germany \\
\hline Restriction endonucleases & New England Biolabs, Frankfurt, Germany \\
\hline RNase A & $\begin{array}{l}\text { Sigma-Aldrich Chemie GmbH, Steinheim, } \\
\text { Germany }\end{array}$ \\
\hline SuperScript III Reverse Transcriptase & Invitrogen, Karlsruhe, Germany \\
\hline T4 DNA Ligase & New England Biolabs, Frankfurt, Germany \\
\hline
\end{tabular}




\subsection{Antibodies}

\begin{tabular}{|c|c|}
\hline rabbit anti-CD55 HPA 02190 & Sigma-Aldrich, Steinheim, Germany \\
\hline HRP labeled anti-rabbit A9161 & Sigma-Aldrich, Steinheim, Germany \\
\hline rabbit anti-CD46 SC9098 & Santa Cruz Biotech., Santa Cruz, USA \\
\hline goat anti-CD59 antibody (C-19) sc-7076 & Santa Cruz Biotech., Santa Cruz, USA \\
\hline donkey anti-goat IgG-HRP sc-2020 & Santa Cruz Biotech., Santa Cruz, USA \\
\hline $\begin{array}{l}\text { mouse monoclonal anti-GAPDH antibody } \\
\text { \#G8795 }\end{array}$ & Sigma-Aldrich, Steinheim, Germany \\
\hline rabbit anti-mouse IgG H\&L (HRP) ab6728 & Abcam, Cambridge, UK \\
\hline $\begin{array}{l}\text { mouse monoclonal IgG1 H01 antibody (D- } \\
\text { 8) sc-136961 }\end{array}$ & Santa Cruz Biotech., Santa Cruz, USA \\
\hline Anti-human CD46-PE (TRA-2-10) & BioLegend, San Diego, USA \\
\hline Anti-human CD55-bio (IA10) & BD Bioscience, San Jose, USA \\
\hline Anti-human CD59-PE (P282) & BioLegend, San Diego, USA \\
\hline Anti-porcine CD62e FITC (1.2B6) & Acris, San Diego, USA \\
\hline Anti-porcine MHC class-II (MSA-3) & Prof. Saalmüller, Wien, Austria \\
\hline Streptavidin-PE & BD Bioscience, San Jose, USA \\
\hline Goat anti-mouse IgG-FITC & Dianova, Hamburg, Germany \\
\hline
\end{tabular}

\subsection{Kits}

\begin{tabular}{ll}
\hline CytoBuster Protein Extraction Reagent & $\begin{array}{l}\text { Novagen/Merck Bioscience, Darmstadt, } \\
\text { Germany }\end{array}$ \\
\hline DNAeasy Blood and Tissue Kit & Qiagen, Hilden, Germany \\
\hline $\begin{array}{l}\text { GenElute Mammalian Genomic DNA } \\
\text { Miniprep Kit }\end{array}$ & $\begin{array}{l}\text { Sigma-Aldrich Chemie GmbH, Steinheim, } \\
\text { Germany }\end{array}$ \\
\hline High Pure RNA Isolation Kit & $\begin{array}{l}\text { Roche Diagnostic GmbH, Mannheim, } \\
\text { German }\end{array}$ \\
\hline Innu Speed Tissue RNA Kit & Analytic Jena, Jena, Germany \\
\hline Lipofectamin 2000 & Invitrogen, Karlsruhe, Germany \\
\hline Mammalian Cell Lysis Kit & $\begin{array}{l}\text { Sigma-Aldrich Chemie GmbH, Steinheim, } \\
\text { Germany }\end{array}$ \\
\hline NucleoBond Xtra Plasmid Purification & $\begin{array}{l}\text { Macherey-Nagel GmbH and Co. KG, Dueren, } \\
\text { Germany }\end{array}$ \\
\hline RNAeasy Minikit & Qiagen, Hilden, Germany \\
\hline Sure Prep RNA/DNA/Protein Purification & $\begin{array}{l}\text { Fisher Scientific, Schwerte, Germany } \\
\text { Kit }\end{array}$ \\
\hline TaqMan Fast Universal PCR Master Mix & Applied Biosystems, Darmstadt, Germany \\
\hline Turbo DNA-free & Ambion, Huntingdon, UK \\
\hline Wizzard SV Gel and PCR Clean Up System & Promega, Mannheim, Germany \\
\hline
\end{tabular}




\subsection{Cells}

\subsubsection{Bacterial strains}

\begin{tabular}{ll}
\hline E. Coli DH10B ElectroMAX & Invitrogen, Karlsruhe, Germany \\
& F- mcrA $\Delta$ (mrr-hsdRMS-mcrBC) $\Phi 80$ lacZ $\Delta$ M15 \\
& $\Delta$ lacX74 recA1 endA1 araD139 $\Delta$ (ara, leu)7697 galU \\
& galK $\lambda$ - rpsL nupG \\
\hline E. Coli SW106 & Prof. Dr. Wolf, Gene Center LMU, Munich, Germany \\
& DH10B cl857, (cro-bioA)<>araC-PBAD Cre, gal490, \\
& gal+, $\Delta$ gakK DH10B cI857, (cro-bioA)<>araC-PBAD Cre, \\
& gal490, gal+, $\Delta$ gakK
\end{tabular}

\subsubsection{Eukaryotic cell lines}

\begin{tabular}{|c|c|}
\hline ST007 & $\begin{array}{l}\text { Cell line } 007 \text {, derived from a porcine lung melanoma; } \\
\text { Prof. Wanke, St. Leon-Roth, Germany }\end{array}$ \\
\hline EHG6k & $\begin{array}{l}\text { HT1080 cells (HPRT-) containing TTE1-HAC with egfp } \\
\text { and HPRT (Kraner } \text { et al., 2007) }\end{array}$ \\
\hline HeLa & Human cell line derived from cervical cancer cells \\
\hline NTERA & $\begin{array}{l}\text { NTERA-2; Stem cell-like embryonic carcinoma cell line, } \\
\text { neuronal precursor }\end{array}$ \\
\hline SCP1 & Human immortalized MSC cell line \\
\hline $\begin{array}{l}\text { Porcine MSC (several isolation } \\
\text { numbers) }\end{array}$ & Chair of Animal Biotechnology, Freising, Germany \\
\hline $\begin{array}{l}\text { Porcine AdMSC } \quad \text { (several } \\
\text { isolation numbers) }\end{array}$ & Chair of Animal Biotechnology, Freising, Germany \\
\hline
\end{tabular}

\subsection{Primers}

Primer sequences are attached in the supplementary part. 


\section{7 qPCR probes}

\begin{tabular}{ll}
\hline hCD46 & FAM-CAAGCAGTCCCTGCAAATG-BHQ \\
\hline hCD55 & FAM-AGGGATGCAGAATTTAGCCTTGTTGG-BHQ \\
\hline hCD59 & FAM-TCAGGTCATAGCCTGCAGTG-BHQ \\
\hline hCD39 & FAM-GTGGTTCAGCATAGTCCCA-BHQ \\
\hline po GAPDH & FAM-AACTCCCTCAAGATCGT-BHQ \\
\hline hGAPDH & FAM-AACAGCCTCAAGATCAT-BHQ \\
\hline
\end{tabular}

\subsection{Ladders}

\begin{tabular}{ll}
\hline 100 bp DNA ladder & New England Biolabs, Frankfurt, Germany \\
\hline 1 kb DNA ladder & New England Biolabs, Frankfurt, Germany \\
\hline Biotinylated protein ladder & Cell Signaling Technol., Danvers, MA, USA \\
\hline MidRange DNA marker II & New England Biolabs, Frankfurt, Germany \\
\hline
\end{tabular}

\subsection{Vectors}

\subsubsection{Bacterial Artificial Chromosomes}

\begin{tabular}{ll}
\hline CD46 BAC: RP11-99A19 & Source BioScience, Nottingham, UK \\
\hline CD55 BAC: clone RP11-357P18 & Source BioScience, Nottingham, UK \\
\hline CD59 PAC: RP4-541C22 & Source BioScience, Nottingham, UK \\
\hline
\end{tabular}

\subsubsection{Plasmids}

\begin{tabular}{ll}
\hline pTSG1-H01 & FLI, Prof. Niemann, Mariensee, Germany \\
\hline CAG-A20 & FLI, Prof. Niemann, Mariensee, Germany \\
\hline hTMmod2 & LMU, Prof. Wolf, München, Germany \\
\hline sCAG-LEA29Y & LMU, Prof. Wolf, München, Germany \\
\hline Pmi/egfp & TUM, Plasmid stock, Chair of Animal \\
& Biotechnology, Freising, Germany \\
\hline PL452 & TUM, Plasmid stock, Chair of Animal \\
PL452 inf & Biotechnology, Freising, Germany \\
\hline pcDNA3.1-hygro(+) & ThermoFisher Scientific \\
\hline
\end{tabular}




\subsection{Tissue culture media and supplements}

\begin{tabular}{ll}
\hline Accutase & $\begin{array}{l}\text { Sigma-Aldrich Chemie GmbH, Steinheim, } \\
\text { Germany }\end{array}$ \\
\hline Advanced DMEM & Gibco Life Technologies, Carlsbad, CA, USA \\
\hline Amphotericin B solution & PAA Laboratories, Pasching, Austria \\
\hline Blasticidin S (BS) & InvivoGen, San Diego, USA \\
\hline Cell culture water & PAA Laboratories, Pasching, Austria \\
\hline Chicken serum & PAA Laboratories, Pasching, Austria \\
\hline DMEM High Glucose (4.5 g/l) & PAA Laboratories, Pasching, Austria \\
\hline DMSO & $\begin{array}{l}\text { Sigma-Aldrich Chemie GmbH, Steinheim, } \\
\text { Germany }\end{array}$ \\
\hline Dulbeco's PBS & PAA Laboratories, Pasching, Austria \\
\hline Fetales calf serum (FCS) & PAA Laboratories, Pasching, Austria \\
\hline G418 & PAA, Pasching, Austria \\
\hline GlutaMAX & Gibco BRL, Paisley, UK \\
\hline Hypoosmolar buffer & Eppendorf, Hamburg, Germany \\
\hline Hygromycin & AppliChem, Darmstadt, Germany \\
\hline Lipofectamine 2000 & Invitrogen, Karlsruhe, Germany \\
\hline MEM- Non Essential Amino Acids & PAA Laboratories, Pasching, Austria \\
\hline Opti-MEM & Gibco Life Technologies, Carlsbad, CA, USA \\
\hline Penicillin/ Streptomycin & Sigma-Aldrich, Deisenhofen, Germany \\
\hline Trypsin-EDTA & PAA, Pasching, Austria \\
\hline $\begin{array}{l}\text { X-Gal (5-Bromo-4-chloro-3-indolyl- } \beta-\text { D- } \\
\text { galactopyranoside) }\end{array}$ & $\begin{array}{l}\text { Sigma-Aldrich Chemie GmbH, Steinheim, } \\
\text { Germany }\end{array}$ \\
\hline
\end{tabular}

\subsection{Bacterial buffers and supplements}

\begin{tabular}{ll}
\hline Ampicillin (Amp) & $\begin{array}{l}\text { Sigma-Aldrich Chemie GmbH, Steinheim, } \\
\text { Germany }\end{array}$ \\
\hline Chloramphenicol & $\begin{array}{l}\text { Sigma-Aldrich Chemie GmbH, Steinheim, } \\
\text { Germany }\end{array}$ \\
\hline Kanamycin (Kana) & $\begin{array}{l}\text { Sigma-Aldrich Chemie GmbH, Steinheim, } \\
\text { Germany }\end{array}$ \\
\hline LB Agar & $\begin{array}{l}\text { Becton Dickinson, Erembodegem-Aalst, } \\
\text { Belgium }\end{array}$ \\
\hline
\end{tabular}

\subsection{Buffers and solutions}

\begin{tabular}{lll}
\hline Antibody dilution buffer & $10 x$ PBS & $4 \mathrm{ml}$ \\
(Immunostaining) & $\mathrm{dH}_{2} \mathrm{O}$ & $36 \mathrm{ml}$ \\
\hline
\end{tabular}




\begin{tabular}{|c|c|c|}
\hline & $\begin{array}{l}\text { BSA } \\
\text { Triton X-100 }\end{array}$ & $\begin{array}{l}0.4 \mathrm{~g} \\
120 \mu \mathrm{l}\end{array}$ \\
\hline APS $10 \%$ & $\begin{array}{l}\text { APS } \\
\mathrm{dH}_{2} \mathrm{O}\end{array}$ & $\begin{array}{l}1 \mathrm{~g} \\
\text { add to } 10 \mathrm{ml}\end{array}$ \\
\hline $\begin{array}{l}\text { Blocking buffer } \\
\text { (Immunostaining) }\end{array}$ & $\begin{array}{l}10 x \text { PBS } \\
\text { Serum } \\
\text { Triton X-100 } \\
\mathrm{dH}_{2} \mathrm{O}\end{array}$ & $\begin{array}{l}2.5 \mathrm{ml} \\
1.25 \mathrm{ml} \\
75 \mu \mathrm{l} \\
21.25 \mathrm{ml}\end{array}$ \\
\hline $\begin{array}{l}\text { Blocking buffer } 5 \% \text { BSA } \\
\text { (Western Blot) }\end{array}$ & $\begin{array}{l}\text { BSA } \\
1 \mathrm{x} \text { TBST }\end{array}$ & $\begin{array}{l}5 \mathrm{~g} \\
\text { add to } 100 \mathrm{ml}\end{array}$ \\
\hline $\begin{array}{l}\text { Blocking buffer } 5 \% \text { skim } \\
\text { milk (Western Blot) }\end{array}$ & $\begin{array}{l}\text { Tris-HCl } \\
\text { NaCl } \\
\text { Skim milk powder } \\
\text { Tween } 20\end{array}$ & $\begin{array}{l}10 \mathrm{mM} \\
150 \mathrm{mM} \\
5 \% \\
0.5 \%\end{array}$ \\
\hline Ficoll-loading dye & $\begin{array}{l}\text { Ficoll PM } 400 \\
\text { Orange G } \\
\mathrm{dH}_{2} \mathrm{O} \\
\end{array}$ & $\begin{array}{l}7.5 \mathrm{~g} \\
0.1 \mathrm{~g} \\
30 \mathrm{ml} \\
\end{array}$ \\
\hline Lämmli buffer (DTT) 4x & $\begin{array}{l}0.5 \text { M Tris-Cl, pH } 6.8 \\
10 \% \text { SDS } \\
\text { Saccharose } \\
1 \text { M Dithiotreitol } \\
\text { Bromphenol blue } \\
\mathrm{dH}_{2} \mathrm{O}\end{array}$ & $\begin{array}{l}5 \mathrm{ml} \\
4 \mathrm{ml} \\
4 \mathrm{~g} \\
260 \mu \mathrm{l} \\
100 \mu \mathrm{l} \\
\text { add to } 10 \mathrm{ml}\end{array}$ \\
\hline Running buffer $10 \mathrm{x}$ & $\begin{array}{l}\text { Tris } \\
\text { Glycine } \\
\text { SDS } \\
\mathrm{dH}_{2} \mathrm{O} \\
\end{array}$ & $\begin{array}{l}30 \mathrm{~g} \\
144 \mathrm{~g} \\
10 \mathrm{~g} \\
\text { add to } 1 \mathrm{l}\end{array}$ \\
\hline Miniprep solution I & $\begin{array}{l}\text { Sucrose } \\
\text { EDTA } \\
\text { Tris (pH8) }\end{array}$ & $\begin{array}{l}5 \mathrm{mM} \\
10 \mathrm{mM} \\
25 \mathrm{mM} \\
\end{array}$ \\
\hline Miniprep solution II & $\begin{array}{l}\mathrm{NaOH} \\
\mathrm{SDS}\end{array}$ & $\begin{array}{l}0.2 \mathrm{M} \\
1 \% \\
\end{array}$ \\
\hline Miniprep solution III & Sodium acetate (pH 4.8) & $3 \mathrm{M}$ \\
\hline $\begin{array}{l}\text { Sodium citrate buffer } 10 \\
\mathrm{mM}\end{array}$ & $\begin{array}{l}\mathrm{C}_{6} \mathrm{H}_{5} \mathrm{Na}_{3} \mathrm{O}_{7} \times 2 \mathrm{H}_{2} \mathrm{O} \\
\mathrm{H}_{2} \mathrm{O}\end{array}$ & $\begin{array}{l}2.94 \mathrm{~g} \\
\text { add to } 1 \mathrm{l} \\
\text { adjust to } \mathrm{pH} 6.0\end{array}$ \\
\hline PBS 10x & $\begin{array}{l}\mathrm{NaCl} \\
\mathrm{KCl} \\
\mathrm{Na}_{2} \mathrm{HPO}_{4} \\
\mathrm{KH}_{2} \mathrm{PO}_{4} \\
\mathrm{dH}_{2} \mathrm{O}\end{array}$ & $\begin{array}{l}80 \mathrm{~g} \\
2 \mathrm{~g} \\
14.4 \mathrm{~g} \\
2.4 \mathrm{~g} \\
\text { add to } 1 \mathrm{l} \\
\text { adjust to } \mathrm{pH} 7.4\end{array}$ \\
\hline Ponceau S & $\begin{array}{l}\text { Ponceau S } \\
100 \% \text { Acetic acid } \\
\mathrm{dH}_{2} \mathrm{O}\end{array}$ & $\begin{array}{l}1 \mathrm{~g} \\
2 \mathrm{ml} \\
\text { add to } 200 \mathrm{ml}\end{array}$ \\
\hline SDS $10 \%$ & $\begin{array}{l}\mathrm{SDS} \\
\mathrm{dH}_{2} \mathrm{O}\end{array}$ & $\begin{array}{l}10 \mathrm{~g} \\
\text { add to } 100 \mathrm{ml}\end{array}$ \\
\hline Semi-Dry Blotting buffer & Tris & $3.03 \mathrm{~g}$ \\
\hline
\end{tabular}




\begin{tabular}{|c|c|c|}
\hline $1 \mathrm{x}$ & $\begin{array}{l}\text { Glycine } \\
\text { Methanol } \\
\mathrm{dH}_{2} \mathrm{O} \\
\end{array}$ & $\begin{array}{l}14.4 \mathrm{~g} \\
200 \mathrm{ml} \\
\text { add to } 1 \mathrm{l} \\
\end{array}$ \\
\hline \multirow[t]{3}{*}{ TAE buffer $50 x$} & Acetic acid $100 \%$ & $2 \mathrm{M}$ \\
\hline & Tris & $2 \mathrm{M}$ \\
\hline & EDTA & $50 \mathrm{mM}$ \\
\hline \multirow[t]{4}{*}{ TBE buffer 10x (pH 8.0) } & Tris & $0.9 \mathrm{M}$ \\
\hline & Boric acid & $0.9 \mathrm{M}$ \\
\hline & EDTA & $20 \mathrm{mM}$ \\
\hline & Acetic acid $100 \%$ & adjust to $\mathrm{pH}=8.0$ \\
\hline \multirow[t]{3}{*}{ TBS 10x } & Tris & $24.2 \mathrm{~g}$ \\
\hline & $\mathrm{NaCl}$ & $80 \mathrm{~g}$ \\
\hline & $\mathrm{dH}_{2} \mathrm{O}$ & add to $1 \mathrm{l}$ \\
\hline \multirow[t]{3}{*}{ TBST $1 \mathrm{x}$} & $10 x \mathrm{TBS}$ & $100 \mathrm{ml}$ \\
\hline & Tween 20 & $1 \mathrm{ml}$ \\
\hline & $\mathrm{dH}_{2} \mathrm{O}$ & add to $1 \mathrm{l}$ \\
\hline \multirow[t]{2}{*}{ TE buffer } & Tris-HCl & $10 \mathrm{mM}$ \\
\hline & EDTA & $1 \mathrm{mM}$ \\
\hline \multirow[t]{4}{*}{ Tris-HCl 0.5 M (pH 6.8) } & Tris- HCl & $15.14 \mathrm{~g}$ \\
\hline & $\mathrm{dH}_{2} \mathrm{O}$ & $125 \mathrm{ml}$ \\
\hline & $\mathrm{HCl}$ & adjust to $\mathrm{pH} 6.8$ \\
\hline & $\mathrm{dH}_{2} \mathrm{O}$ & add to $250 \mathrm{ml}$ \\
\hline \multirow[t]{4}{*}{ Tris-HCl 1 M (pH 8.8) } & Tris & $39.4 \mathrm{~g}$ \\
\hline & $\mathrm{dH}_{2} \mathrm{O}$ & $125 \mathrm{ml}$ \\
\hline & $\mathrm{HCl}$ & adjust to $\mathrm{pH} 8.8$ \\
\hline & $\mathrm{dH}_{2} \mathrm{O}$ & add to $250 \mathrm{ml}$ \\
\hline \multirow[t]{3}{*}{ TTE buffer } & Tris pH 8.5 & $20 \mathrm{mM}$ \\
\hline & Triton X 100 & $1 \%$ \\
\hline & EDTA pH 7.8 & $2 \mathrm{mM}$ \\
\hline
\end{tabular}

\subsection{Laboratory equipment}

\begin{tabular}{ll}
\hline Accu-Jet & Brand, Dietenhofen, Germany \\
\hline Biofuge fresco 3324 rotor & Heraeus Instruments, Hanau, Germany \\
\hline Bio Imaging System Gene Genius & Syngene, Cambridge, UK \\
\hline Camera AxioCam MR (Axiovision 4.2) & Carl Zeiss Jena GmbH, Jena, Germany \\
\hline Cell counting chamber & Neubauer, Marienfeld, Germany \\
\hline Centrifuge Megafuge 2.0R/Varifuge 3.0R & Heraeus Instruments, Hanau, Germany \\
\hline Centifuge 5810/Minispin & Eppendorf, Hamburg, Germany \\
\hline Centrifuge 4K15C, 1-15 & Sigma, Osterode, Germamny \\
\hline Centrifuge J2-HS & Beckman Coulter GmbH, Krefeld, \\
& Germany
\end{tabular}




\begin{tabular}{|c|c|}
\hline $\mathrm{CO}_{2}$ Incubator & Forma Scientific Inc., Marietta, Ohio, USA \\
\hline Cooling unit: Freezer $-20^{\circ} \mathrm{C}$ & Liebherr International, Bulle, Switzerland \\
\hline Cooling unit: Freezer $-80^{\circ} \mathrm{C}$ & $\begin{array}{llll}\text { Thermo } & \text { Electron } \mathrm{GmbH}, \text { Dreieich, } \\
\text { Germany } & & & \end{array}$ \\
\hline Cryo Freezing device & Qualilab, Olivet, France \\
\hline Digital Graphis Pronter UP D895MD & Syngene, Cambridge, UK \\
\hline Electroporation cuvettes & $\begin{array}{l}\text { PEQLAB Biotechnologie GmbH, Erlangen, } \\
\text { Germany }\end{array}$ \\
\hline Electroporation: Multiporator & Eppendorf, Hamburg, Germany \\
\hline FACSCalibur flow cyotometer & BD Bioscience, San Jose, USA \\
\hline Gene Pulser II & $\begin{array}{l}\text { Bio-Rad Labaratories GmbH, München, } \\
\text { Germany }\end{array}$ \\
\hline $\begin{array}{l}\text { Gel electrophoresis- } \\
\text { CSSU78/CSSU1214 }\end{array}$ & $\begin{array}{l}\text { Thermo Electron GmbH, Dreieich, } \\
\text { Germany }\end{array}$ \\
\hline Glassware & $\begin{array}{l}\text { Marienfeld GmbH, Lauda-Königshofen, } \\
\text { Germany }\end{array}$ \\
\hline Hera Safe clean bench & Heraeus Instruments, Hanau, Germany \\
\hline Incubator & Binder GmbH, Tuttlingen, Germany \\
\hline Incubator: Forma orbital shaker & $\begin{array}{llll}\text { Thermo } & \text { Electron } \mathrm{GmbH}, \text { Dreieich, } \\
\text { Germany } & & & \end{array}$ \\
\hline Ice machine & Eurfrigor, Lainate, Italy \\
\hline Microbeta scintillation counter & Wallac, Turku, Finland \\
\hline Microscope Axiovert 25/135 & Carl Zeiss Jena GmbH, Jena, Germany \\
\hline Microwave MDA MW12M706-AU & $\begin{array}{l}\text { MHA Haushaltswaren GmbH, Barsbüttel, } \\
\text { Germany }\end{array}$ \\
\hline PCR DNA Engine Dyad Cycler(PTC220) & $\begin{array}{l}\text { Bio-Rad Labaratories GmbH, München, } \\
\text { Germany }\end{array}$ \\
\hline Pipets: Gilson Pipetman $(2,20,200,1000 \mu \mathrm{l})$ & Gilson, Bad Camberg, Germany \\
\hline Pipetus & $\begin{array}{l}\text { Hirschmann Laborgeräte, Eberstadt, } \\
\text { Germany }\end{array}$ \\
\hline Power supply EC105 & $\begin{array}{lll}\text { Thermo Electron } \mathrm{GmbH}, & \text { Dreieich, } \\
\text { Germany } & & \end{array}$ \\
\hline
\end{tabular}




\begin{tabular}{|c|c|}
\hline Power supply Power PAC 300 & $\begin{array}{l}\text { Bio-Rad Laboratories GmbH, München, } \\
\text { Germany }\end{array}$ \\
\hline Safety Cabinets HERAsafe HSP & Heraeus Instruments, München, Germany \\
\hline Spectrophotometer: Biophotometer & Eppendorf, Hamburg, Germany \\
\hline TaqMan 7500 Fast Real-Time PCR Cycler & Applied Biosystems, Darmstadt, Germany \\
\hline UV-Photometer DU-640 & $\begin{array}{llll}\text { Beckman } & \text { Coulter GmbH, } \\
\text { Germany } & & & \\
\end{array}$ \\
\hline Vortex Mixer & VELP Scientifica, Milano, Italy \\
\hline Water bath Haake C10-K15 & $\begin{array}{l}\text { Thermo Fisher Scientific, Karlsruhe, } \\
\text { Germany }\end{array}$ \\
\hline
\end{tabular}

\subsection{Consumables}

\begin{tabular}{ll}
\hline Cell culture flasks & Corning Inc., New York, USA \\
\hline Cell culture plates & Corning Inc., New York, USA \\
\hline Centrifuge tubes $(15 \mathrm{ml}, 50 \mathrm{ml})$ & Corning Inc., New York, USA \\
\hline Cryo Tube vials & Nunc, Wiesbaden-Biebrich, Germany \\
\hline Electroporation cuvettes & Peqlab Biotechnologie GmbH, Erlangen, \\
& Germany \\
\hline Glassware & Marienfeld GmbH, Landa, Germany \\
\hline MicroAmp fast Optical 96-Well Plate & Applied \\
& Germany \\
\hline Petri dishes & Brand GmbH \& Co. KG, Wertheim, \\
& Germany \\
\hline Photometer cuvettes & Eppendorf, Hamburg, Germany \\
\hline Pipet tips & Brand GmbH \& Co. KG, Wertheim, \\
\hline Plastic pipets (sterile) & Germany \\
\hline Reaction tubes (1.5 ml, 2 ml) & Corning Inc., New York, USA \\
\hline Stericup & Brand GmbH \& Co. KG, Wertheim, \\
\hline
\end{tabular}




\subsection{Software}

\begin{tabular}{ll}
\hline AxioVision & Zeiss AG, Oberkochen, Germany \\
\hline Clustal Omega & www.ebi.ac.uk/Tools/msa/clustalo \\
\hline Enzyme finder & www.neb.com \\
\hline everyVECTOR & www.everyvector.com \\
\hline GeneCards & www.genecards.org \\
\hline Primer 3 & bioinfo.ut.ee/primer3-0.4.0/primer3 \\
\hline Reverse Complement & www.bioinformatics.org/sms/rev_comp.html \\
\hline UniProt & www.uniprot.org/uniprot \\
\hline CellQuest Pro & DB Bioscience, San Jose, USA \\
\hline
\end{tabular}

\subsection{Glycerol stocks of bacteria}

For long-term storage of bacteria a glycerol stock was generated by combining one $\mathrm{ml}$ of a $\mathrm{LB}$ over night culture with $0.5 \mathrm{ml} \mathrm{99 \%} \mathrm{Glycerol} \mathrm{and} \mathrm{stored} \mathrm{at}-80^{\circ} \mathrm{C}$.

\subsection{Isolation of plasmid DNA}

Due to the amount of required DNA different methods were used for isolation. Small amounts were isolated using the miniprep protocol. For larger amounts of DNA with a higher purity, a midiprep or maxiprep was conducted.

\subsubsection{Miniprep}

A single bacteria clone was picked from an LB plate and put into LB media with required antibiotics. After incubation over night at $37^{\circ} \mathrm{C}$ in an orbital shaker at $200 \mathrm{rpm}$, two $\mathrm{ml}$ of the solution were centrifuged for one minute at $14000 \mathrm{rpm}$. The supernatant was removed and the pellet was resuspended using $100 \mu \mathrm{l}$ of solution I. 
Alkaline lysis was carried out by adding $200 \mu \mathrm{l}$ of solution II and gently mixing. After incubation for three minutes at room temperature, $150 \mu \mathrm{l}$ of solution III were added. The tube was inverted several times and incubated for 30 minutes on ice. Cell debris was separated from the DNA solution by centrifugation for five minutes at $14000 \mathrm{rpm}$. The supernatant was transferred into a new tube and the DNA was precipitated adding one $\mathrm{ml}$ of 95\% ethanol and centrifugation for 15 minutes at $14000 \mathrm{rpm}$. Washing of the pellet was done with $80 \%$ and $95 \%$ ethanol and centrifugation for ten minutes at $14000 \mathrm{rpm}$ between each step. Ethanol was removed and the pellet was dried at room temperature and resuspended in $50 \mu \mathrm{l}$ of $\mathrm{H}_{2} \mathrm{O}$ supplemented with RNase A.

\subsubsection{Midiprep and Maxiprep}

For higher amounts of DNA with a higher purity and for cell culture transfection experiments midi- or maxi scale plasmid DNA isolation was performed using the NucleoBond Xtra Midi Kit or NucleoBond Xtra Maxi Kit according to the manufacturer's "Low-copy plasmid purification" protocol. $250 \mathrm{ml}$ or $500 \mathrm{ml}$ of over night culture were used for plasmid isolation.

\subsection{Recombineering competent SW106}

The recombineering protocol was performed according to Liu et al., 2003. For recombination of the $\mathrm{BAC}$ and $\mathrm{PAC}$ constructs containing the full length genomic sequence of the complement inhibitory genes, the SW106 strains were grown at $32^{\circ} \mathrm{C}$. Five $\mathrm{ml}$ of an over night culture were added to $500 \mathrm{ml}$ of $\mathrm{LB}$ medium containing $12.5 \mu \mathrm{g} / \mathrm{ml}$ Chloramphenicol and bacteria were grown until $\mathrm{OD}_{600}$ reached 0.3-0.4. Bacteria were incubated for 15 minutes at $42^{\circ} \mathrm{C}$ and harvested by centrifugation for ten minutes at $4000 \mathrm{rpm}$ and $4^{\circ} \mathrm{C}$. The supernatant was removed and the pellet resuspended in $50 \mathrm{ml}$ ice-cold water followed by centrifugation for ten minutes at $4000 \mathrm{rpm}$ and $4^{\circ} \mathrm{C}$. Washing steps were repeated using $20 \mathrm{ml}$ of ice-cold water and afterwards five $\mathrm{ml}$ of ice-cold $10 \%$ glycerol/water. 
After centrifugation the supernatant was removed and the pellet was resuspended in one $\mathrm{ml}$ of ice-cold $10 \%$ glycerol/water. Aliquots of $50 \mu \mathrm{l}$ were made and stored at $-80^{\circ} \mathrm{C}$ or directly used for electroporation.

For homologous recombination bacteria were mixed with one ng of linearized plasmid DNA, electroporated at $1.8 \mathrm{kV}$ for five $\mathrm{ms}$ and stored at $32^{\circ} \mathrm{C}$ for one hour. After centrifugation for five minutes at $5000 \mathrm{rpm}$, the supernatant was almost completely removed, bacteria resuspended and plated on LB plates containing antibiotics.

\subsection{DNA restriction digest}

To confirm DNA cloning steps and verify plasmid lengths, analytical digests were performed using two to three $\mu$ g DNA. For preparative digests about eight to 15 $\mu \mathrm{g}$ DNA were used. All digests were performed according to the manufacturer's instructions. Table one shows an exemplary analytical digest.

Table 1: Analytical digest

\begin{tabular}{ll}
\hline DNA & $2-3 \mu \mathrm{g}$ \\
\hline $10 \mathrm{x}$ NEB Buffer & $5 \mu \mathrm{l}$ \\
\hline $10 \mathrm{X}$ BSA & $5 \mu \mathrm{l}$ \\
\hline $\mathrm{H}_{2} \mathrm{O}$ & add up to $50 \mu \mathrm{l}$ \\
\hline Enzyme & $3 \mathrm{U} / \mu \mathrm{g}$ \\
\hline
\end{tabular}

\subsection{Blunting of DNA fragments}

Some cloning steps require blunt ends for the ligation of the vector and the insert. Blunting was performed using DNA Polymerase I Large Fragment (Klenow), which fills in $5^{\prime}$ overhangs and removes $3^{\prime}$ overhangs to form blunt ends. DNA polymerase I is active in all NEB buffers but requires the addition of dNTPs to inhibit its $3^{\prime}-5^{\prime}$ exonuclease activity. Table two shows an exemplary blunting digest. The reaction was performed for 15 minutes at $25^{\circ} \mathrm{C}$ and stopped by addition of EDTA to a final concentration of at least $10 \mathrm{mM}$. 
Table 2: Blunting digest

\begin{tabular}{ll}
\hline DNA & $2-10 \mu \mathrm{g}$ \\
\hline $10 \mathrm{x}$ NEB Buffer & $5 \mu \mathrm{l}$ \\
\hline dNTPs 2mM & $1.5 \mu \mathrm{l}$ \\
\hline $\mathrm{H}_{2} \mathrm{O}$ & add up to $50 \mu \mathrm{l}$ \\
\hline Klenow (Stock $5 \mathrm{U} / \mathrm{ul})$ & $1 \mathrm{U} / \mu \mathrm{g}$ DNA \\
\hline
\end{tabular}

\subsection{Dephosphorylation of cleaved DNA}

To inhibit religation in case of blunt or compatible ends, the $5^{\prime}$ phosphates were removed using Calf Intestinal Alkaline Phosphatase (CIP). Two $\mu$ l of CIP were added to a $50 \mu \mathrm{l}$ restriction digest and incubated for 30 minutes at $37^{\circ} \mathrm{C}$.

\subsection{Ligation}

To ligate vector backbones and fragments, T4 ligase was used. This enzyme catalyzes the formation of a phosphodiester bond between $5^{\prime}$ phosphate and $3^{\prime}$ hydroxyl termini. The reaction was performed according to the manufacturer's protocol. The ligation mixture was incubated for two hours at $25^{\circ} \mathrm{C}$ and additionally over night at $4^{\circ} \mathrm{C}$.

\subsection{Electroporation}

Electroporation was performed using the Eppendorf Multiporator according to the manufacturer's protocol. Fifty $\mu \mathrm{l}$ of electrocompetent E.coli DH10b were thawed on iced, mixed with one to five $\mu \mathrm{l}$ of the ligation mixture and electroporated at $2.5 \mathrm{kV}$ for five ms. After 30 minutes of incubation time in an antibiotic-free $\mathrm{LB}$ medium at $37^{\circ} \mathrm{C}$, bacteria were plated on LB plates containing antibiotics and incubated over night at $37^{\circ} \mathrm{C}$. For blue-white screening the plates were additionally coated with X-Gal and IPTG. 


\subsection{Colony PCR}

A colony PCR is a very fast screening method for the detection of successful cloning events. One primer is located in the plasmid backbone, the other is located in the plasmid insert. Single bacterial clones are selected from a LB plate and transferred with a tip into a PCR tube containing $30 \mu \mathrm{l}$ TTE buffer. The tube is heated for five minutes at $95^{\circ} \mathrm{C}$ and centrifuged for ten minutes at $350 \mathrm{~g}$ and $4^{\circ} \mathrm{C}$. Three $\mu \mathrm{l}$ of the solution are used as template for the PCR reaction. The TTE/plasmid solution can be stored at $-20^{\circ} \mathrm{C}$ and used for several months.

\subsection{Agarose gel electrophoresis}

To analyze the size of DNA fragments between $20 \mathrm{bp}$ up to $16 \mathrm{~kb}$, an agarose gel electropohoresis was conducted. For analytical restriction digests 0.8\%-4\% TBE gels containing ethidium bromide were prepared. For a preparative restriction digest $0.8 \%$ TAE gels with a reduced amount of ethidium bromide were made. Samples were mixed with $3 x$ loading dye or directly used for gelelectrophoresis with the green PCR GoTaq 5x Buffer. Gels were run for one up to six hours at 80$100 \mathrm{~V}$ according to the expected fragment sizes. DNA fragments were analyzed under UV light at $366 \mathrm{~nm}$ using the Bio Imaging System Gene Genius.

\subsection{Pulsed field gel electrophoresis}

Pulsed field gel electrophoresis (PFGE) is used to separate large DNA fragments $(>20 \mathrm{~kb})$ in an agarose gel according to their size. The voltage is switched permanently among different directions. The switch times depend on the DNA fragment sizes.

To examine the sizes of BAC and PAC vector constructs containing the complement inhibitory genes, $1 \%$ TBE gels with $0.5 \%$ TBE buffer were prepared. The gels were run at $12^{\circ} \mathrm{C}$ for 16 hours at $6 \mathrm{~V}$ and the switch time between was chosen between 1-30s. 


\subsection{Isolation of DNA fragments from agarose-gels}

DNA bands were cut out of the agarose-gel under UV light using a clean scalpel and purified with the Wizard SV Gel and PCR Clean-up System according to the manufacturer's protocol.

\subsection{Determination of DNA and RNA concentration}

DNA and RNA concentrations were measured using the BioPhotometer or the NanoDrop Lite according to the manufacturer's instructions.

\subsection{Polymerase chain reaction (PCR)}

For amplification of plasmid DNA fragments and screening PCRs, GoTaq Polymerase as well as Q5 Polymerase were used. Q5 Polymerase has a more than 100 fold lower error rate compared to GoTaq Polymerase and was advantageous for several samples. In case of genomic templates and long-range PCR reactions 5Prime DNA polymerase was used. Table three gives an overview of the different PCR settings

Table 3: PCR settings

\begin{tabular}{|c|c|c|c|c|c|}
\hline Component & $\begin{array}{c}\text { Final } \\
\text { concentration }\end{array}$ & Step & Temperature & Time & Cycles \\
\hline \multicolumn{6}{|l|}{ GoTaq } \\
\hline DNA & & $\begin{array}{l}\text { Initial } \\
\text { denaturation }\end{array}$ & 98 & $2 \min$ & 1 \\
\hline $\begin{array}{l}10 x \text { green } \\
\text { buffer }\end{array}$ & $1 \mathrm{x}$ & Denaturation & 98 & $45 \mathrm{~s}$ & $30-40$ \\
\hline dNTPs & $200 \mu \mathrm{M}$ each & Annealing & $58-62^{\circ} \mathrm{C}$ & $45 \mathrm{~s}$ & \\
\hline $\begin{array}{l}\text { Forward } \\
\text { primer }\end{array}$ & $0.5 \mu \mathrm{M}$ & Elongation & 72 & $\begin{array}{c}60 \\
\mathrm{~s} / \mathrm{kb}\end{array}$ & \\
\hline $\begin{array}{l}\text { Reverse } \\
\text { primer }\end{array}$ & $0.5 \mu \mathrm{M}$ & $\begin{array}{l}\text { Final } \\
\text { elongation }\end{array}$ & 72 & $5 \mathrm{~min}$ & 1 \\
\hline $\mathrm{H}_{2} \mathrm{O}$ & to $25 \mu \mathrm{l}$ & Storage & 8 & forever & 1 \\
\hline GoTaq & $0.03 \mathrm{U} / \mu \mathrm{l}$ & & & & \\
\hline
\end{tabular}




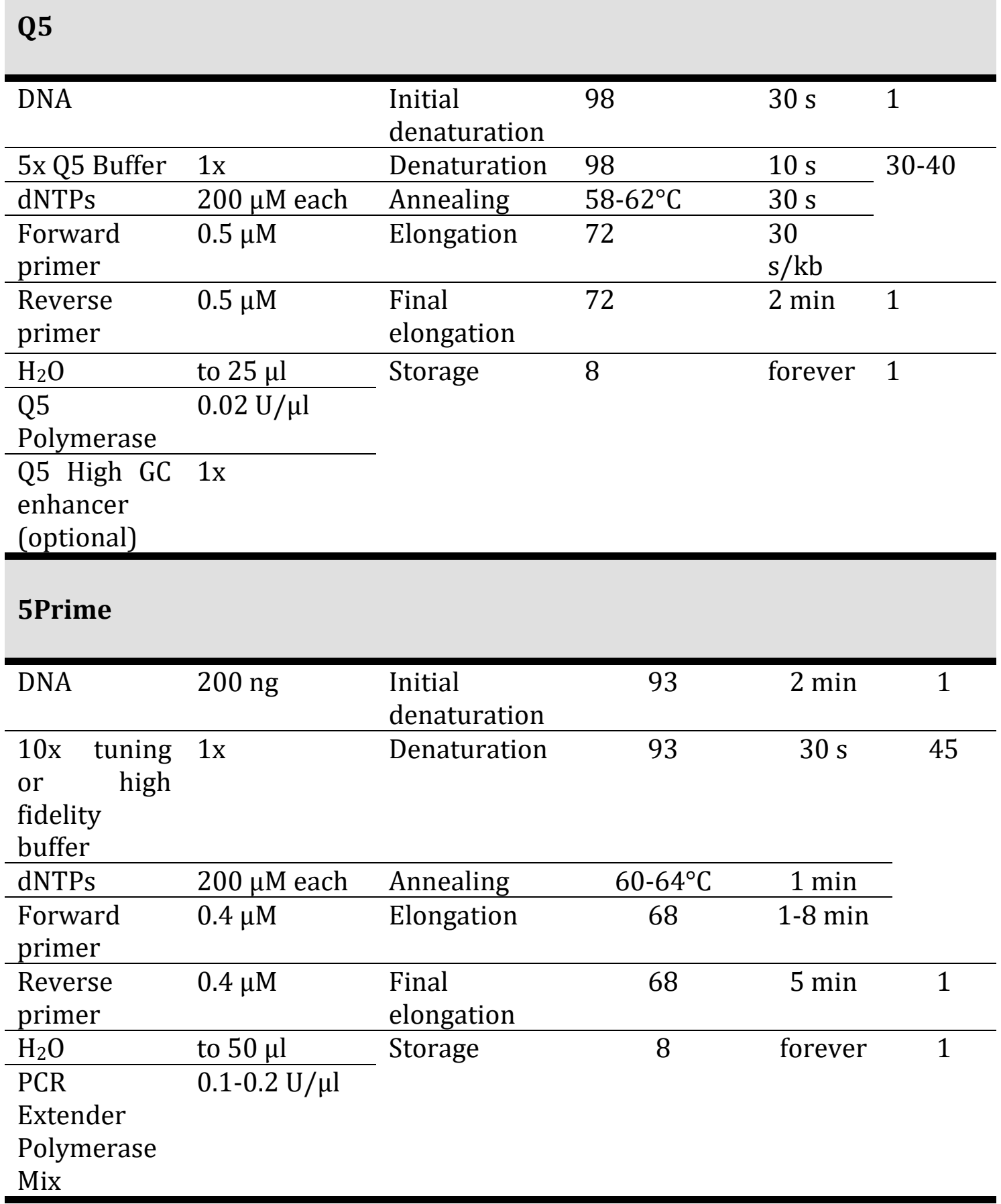

\subsection{Splice Overlap Extension PCR (SOE-PCR)}

A SOE-PCR is used to introduce specific point mutations. Two overlapping primers, containing the desired mutations, are used in separate reactions together with two flanking primers to produce overlapping DNA fragments. 
If these fragments are used as templates for an additional PCR with the flanking primer combination, a full-length mutagenized DNA sequence can be produced. GoTaq Polymerase or Q5 Polymerase was used to perform the single PCR reaction steps.

\subsection{Sequencing}

Sequencing was conducted by MWG Eurofins Operon (Eberberg, Germany).

\subsection{Microcell-mediated chromosome transfer}

To transfer a preformed human artificial chromosome (HAC) from human HT1080 cells (EHG6k) into porcine ST007 cells or porcine mesenchymal stem cells, a microcell-mediated chromosome transfer (MMCT) was conducted. The protocol was modified according to the results of Stubblefield and Perhouse, 1992, Suzuki et al., 2006 and Katoh et al., 2010.

Two T150 flasks with EHG6k cells (about 80\% confluent) were treated for 48 hours with one $\mu \mathrm{g} / \mathrm{ml}$ Colcemid. Cells were trypsinised and centrifuged for five minutes at $340 \mathrm{~g}$. The pellet was resuspended in serum-free DMEM media containing $40 \mu \mathrm{g} / \mathrm{ml}$ Cytochalasin B and $0.5 \mu \mathrm{g} / \mathrm{ml}$ Colcemid. After five minutes incubation time at $37^{\circ} \mathrm{C}$, Percoll was added $1: 2$ and a density gradient centrifugation was performed for 90 minutes at $37^{\circ} \mathrm{C}$ and $19000 \mathrm{~g}$. Microcells were harvested and serum-free DMEM media was added before filtration with a three $\mu \mathrm{m}$ filter. Microcells were centrifuged or 15 minutes at $500 \mathrm{~g}$, the pellet was resuspended with two $\mathrm{ml}$ of serum-free DMEM containing $25 \mu \mathrm{g} / \mathrm{ml}$ PHA-P and added dropwise on the $10 \mathrm{~cm}$ plates with the porcine ST007 cells or MSCs. The cells were incubated with the mixture for 15 minutes at $37^{\circ} \mathrm{C}$. Afterwards the supernatant was removed and about one ml 50\% PEG 1500 was given dropwise on the plate. After $90 \mathrm{~s}$ incubation time, ten $\mathrm{ml}$ of serum-free media were added very slowly. Cells were washed two more times with serum-free media and selected after two days with four $\mu \mathrm{g} / \mathrm{ml}$ BS. Single cell clones were picked, expanded and further analysed. 


\subsection{Fluorescence in situ hybridization}

To detect the transfer of a human artificial chromosome from human into porcine cells and to determine the integration sites of various xenogeneconstructs, a fluorescence in situ hybridization (FISH) was performed.

\subsubsection{Preparation of metaphase chromosome spreads}

For the preparation of metaphase chromosome spreads, cells were treated with $0.1 \mu \mathrm{g} / \mathrm{ml}$ Colcemid for three hours at $37^{\circ} \mathrm{C}$. Cells were detached from the flask and centrifuged together with the supernatant for five minutes at $300 \mathrm{~g}$. The pellet was resuspended dropwise in five ml hypotonic solution $(0.075 \mathrm{M}$ potassium chloride and $0.8 \%$ sodium citrate) and incubated for 15 minutes at room temperature. After adding $0.5 \mathrm{ml}$ of $-20^{\circ} \mathrm{C}$ cold fixative $(3: 1$ methanol and acetic acid), cells were incubated for another ten minutes and centrifuged for five minutes at $300 \mathrm{~g}$. The supernatant was discarded and the pellet was gently resuspended with eight $\mathrm{ml}$ fixative $\left(-20^{\circ} \mathrm{C}\right)$ and centrifuged for five minutes at $300 \mathrm{~g}$ and $4^{\circ} \mathrm{C}$. This washing step was repeated three times. At the end, the pellet was resuspended in $0.2-1 \mathrm{ml}$ fixative $\left(-20^{\circ} \mathrm{C}\right)$ and remained for at least ten minutes at $-20^{\circ} \mathrm{C}$.

The microscope slides were stored over night in ethanol, rinsed with deionized water and placed in a supersonic bath for ten minutes. After rinsing the slides again for ten minutes with deionized water, the slides were covered with membra-pure water and chilled on ice.

The maturated cell suspension was dropped from $40 \mathrm{~cm}$ height onto the wet and cold microscope slides and placed on a slide warmer for five minutes at $37^{\circ} \mathrm{C}$. The slides had to be air-dried for two more weeks under light protection before they could be used for FISH analysis. 


\subsubsection{Probe production}

All probes were produced using nick translation. DNA polymerase I causes single strand breaks which are used as starting point for the $5^{\prime}-3^{\prime}$ exonuclease-activity of DNasel. This way one DNA-strand is degraded, the nick moves forward into synthesis-direction and a new DNA-strand is synthesized with biotin or digoxigenin labeled dUTPs, which are recognized by specific antibodies. As template, a specific region of the gene was chosen. Table four shows a typical nick translation approach.

Table 4: Nick translation

\begin{tabular}{ll}
\hline DNA fragment & $50-200 \mu \mathrm{g}$ \\
\hline NEB2 & $15 \mu \mathrm{l}$ \\
\hline dACG 1mM & $7.5 \mu \mathrm{l}$ \\
\hline dTTP 1mM & $3.75 \mu \mathrm{l}$ \\
\hline Dig-dUTP 1mM & $3.75 \mu \mathrm{l}$ \\
\hline Mercaptoethanol 100 mM & $1 \mu \mathrm{l}$ \\
\hline $\mathrm{H}_{2} \mathrm{O}$ & up to $150 \mu \mathrm{l}$ \\
\hline Polymerase I & $2 \mu \mathrm{l}$ \\
\hline DNase 1:500 & $1.5 \mu \mathrm{l}$ \\
\hline $\mathrm{MgCl}_{2}$ & $2 \mu \mathrm{l}$ \\
\hline
\end{tabular}

The nicktranslation approach was incubated for $90-120$ minutes at $15^{\circ} \mathrm{C}$. The length of the DNA fragments was controlled on a 1\% TBE-agarose-gel. If the desired length of about $500 \mathrm{bp}$ was reached, the mixture was frozen on dry-ice for ten minutes. 1/10x volume of 0.5 M EDTA was added and all enzymes were additionally inactivated for ten minutes at $65^{\circ} \mathrm{C}$. The probes were precipitated with $1 / 10 \mathrm{x}$ volume of five $\mathrm{M} \mathrm{NaCl}$ and $2.5 \mathrm{x}$ volume of ethanol $\left(-20^{\circ} \mathrm{C}\right)$ at $-20^{\circ} \mathrm{C}$ over night. 


\subsubsection{Probes for FISH}

Different probes were prepared to analyze the composition of a xeno-HAC and to identify chromosomal xeno-gene integration sites after cotransfection experiments via FISH analysis (Table 5). Their quality was verified using dot blot analysis and FISH.

Table 5. Probes for the detection of xeno-genes

\begin{tabular}{|c|c|c|c|c|}
\hline Xeno-gene & Dye & Donor-Plasmid & $\begin{array}{l}\text { Fragment for } \\
\text { nicktranslation }\end{array}$ & $\begin{array}{l}\text { Time for } \\
\text { nicktranslation }\end{array}$ \\
\hline LEA29Y & Biotin & $\begin{array}{l}\text { sCAG-LEA } \\
\text { AscI digested }\end{array}$ & $5.3 \mathrm{~kb}$ & 70 minutes \\
\hline A20 & Digoxigenin & $\begin{array}{l}\text { pCAGEhA20 } \\
\text { EcoRI digested }\end{array}$ & $2.5 \mathrm{~kb}$ & 90 minutes \\
\hline $\mathrm{TM}$ & Biotin & $\begin{array}{l}\text { hTMmod2 } \\
\text { SalI,NriI digested }\end{array}$ & $10.5 \mathrm{~kb}$ & 65 minutes \\
\hline CD59 & Digoxigenin & $\begin{array}{l}\text { CD59 M15 1-3, } \\
\text { Not I digested }\end{array}$ & $116 \mathrm{~kb}$ & 335 minutes \\
\hline HO1 & Digoxigenin & $\begin{array}{l}\text { pTSG1-HMOX1 } \\
\text { HindIII digested }\end{array}$ & $1.4 \mathrm{~kb}$ & 48 minutes \\
\hline
\end{tabular}

\subsubsection{Dot blot}

To determine the amount of integrated, labeled dUTPs in the probe, a Dot blot was conducted. Biotin-labeled dUTPs are recognized by steptavidin-labeled antibodies whereas digoxigenin-labeled antibodies are recognized by anti-Digantibodies. Both antibodies are labeled with alkaline phosphatase, which can be detected by the addition of 5-brom-4-chlor-3-indoxylphosphate (BCIP) and nitroblue-tetrazoliumchloride (NBT).

For the Dot blot, a 1:10 and 1:100 dilution of the probe was prepared and one $\mu \mathrm{l}$ was pipetted on a nitrocellulose-paper, air-dried and denatured for one minute with UV-light. 
The paper was humidified with AP1 solution and incubated for ten minutes in AP2 solution at room temperature in a dark chamber. The antibodies were diluted 1:5000 - 1:6000 in AP1 solution and the paper was incubated for ten minutes at room temperature in the dark, washed two times for three minutes each in AP1 solution and three times for three minutes each in AP3 solution. To produce a Dot blot signal, the paper was incubated for up to one hour in the dark in staining solution (Five ml AP3, $22 \mu \mathrm{l} \mathrm{NBT}$ and $16.6 \mu \mathrm{l} \mathrm{BCIP),} \mathrm{washed} \mathrm{with}$ water and air-dried. The intensity of the spots is proportional to the amount of integrated dUTPs in the probes.

\subsubsection{Probe precipitation and denaturation}

According to the actual requirements and the cell type used, probes were mixed with different blocking reagents and precipitated with $0.1 \mathrm{x}$ volume of three $\mathrm{M}$ sodium acetate and $2.5 \mathrm{x}$ volume of ethanol. In most cases blocking reagents such as Cot1, IgH and salmon were used. The precipitated probes were incubated over night at $-20^{\circ} \mathrm{C}$ and centrifuged for ten minutes at $14000 \mathrm{rpm}$ and $4^{\circ} \mathrm{C}$. The pellet was washed with $70 \%$ ethanol $\left(-20^{\circ} \mathrm{C}\right)$ and centrifuged for ten minutes. The supernatant was discarded and the pellet vacuum-dried for five minutes. Probes were resuspended in five $\mu$ f formamide per reaction and incubated for three hours at $37^{\circ} \mathrm{C}$. Five $\mu \mathrm{l}$ of mastermix ( $40 \%$ dextran sulfate in $2 \mathrm{xSSC}$ ) per reaction were added and the probes were denatured for five minutes at $72^{\circ} \mathrm{C}$, incubated for one hour at $37^{\circ} \mathrm{C}$ and stored at $-20^{\circ} \mathrm{C}$.

\subsubsection{RNase treatment and pepsin digestion}

To remove cell debris, RNA and proteins from the microscope slides, a RNase treatment and pepsin digestion was conducted. Therefore the slides were incubated for five minutes in 2xSSC before being covered for 30 minutes at $37^{\circ} \mathrm{C}$ in a humid chamber with $200 \mu \mathrm{l}$ RNase solution $(200 \mu \mathrm{l} 2 \mathrm{xSSC}, 2 \mu \mathrm{l}$ RNase 10 $\mathrm{mg} / \mathrm{ml}$ ). Washing the slides three times for five minutes in 2xSSC stopped RNase treatment. Pepsin was prepared in a $37^{\circ} \mathrm{C}$ warm solution $(100 \mu \mathrm{l} 5 \mathrm{~N} \mathrm{HCl}$ in $50 \mathrm{ml}$ $\mathrm{H}_{2} \mathrm{O}$ ) and the digest was carried out for exactly 15 seconds. 
The microscope slides were removed from the pepsin solution and residual pepsin was diluted by washing for five minutes with PBS. After a rising ethanol series $\left(70 \%, 90 \% 100 \%\right.$ ethanol) at $-20^{\circ} \mathrm{C}$ for three minutes each, microscope slides were air-dried.

Metaphase spreads were denatured to enable efficient probe hybridization for one minute and 45 seconds at $72^{\circ} \mathrm{C}$ in a $70 \%$ formamide/2xSSC solution. A rising ethanol series $\left(70 \%, 90 \%, 100 \%\right.$ ethanol at $\left.-20^{\circ} \mathrm{C}\right)$ for five minutes each ensured fixation of the denatured DNA strands. After the microscope slides were airdried, they were used for fluorescent probe hybridization.

\subsubsection{Probe hybridization}

The denatured probe was used for hybridization with the denatured metaphase spreads. Ten $\mu \mathrm{l}$ of the probe mixture was pipetted on a marked field on the microscope slide and covered with a cover slip which was sealed with glue to prevent evaporation of the probe mixture during denaturation for two minutes at $72^{\circ} \mathrm{C}$. Hybridization of the probe and the DNA strands was performed at $37^{\circ} \mathrm{C}$ over night. The glue and the cover slip were removed and the microscope slides were washed three times at $42^{\circ} \mathrm{C}$ with $2 \mathrm{xSSC}$-Tween, three times at $60^{\circ} \mathrm{C}$ with $1 \mathrm{xSSC}$ and two times at $42^{\circ} \mathrm{C}$ with $2 \mathrm{xSSC}$-Tween for five minutes each.

To prevent unspecific binding of the antibodies, the microscope slides were treated with one $\mathrm{ml} 1 \%$ BSA for 30 minutes at $37^{\circ} \mathrm{C}$. Residual BSA was removed by washing with $2 x S S C$-Tween for five minutes at $37^{\circ} \mathrm{C}$. For the detection of the biotin or digoxigenin labeled probes, $200 \mu \mathrm{l}$ of $1 \%$ BSA were mixed with $0.67 \mu \mathrm{l}$ Cy3.5 and $1.33 \mu$ l sheep antidig-fluorescein, pipetted to the microscope slide and covered with a cover slip. After 45 minutes incubation time at $37^{\circ} \mathrm{C}$ in a humid chamber, excess of antibodies was removed by washing two times with 2xSSC at $42^{\circ} \mathrm{C}$. One $\mathrm{ml}$ of DAPI solution ( $0.5 \mu \mathrm{l}$ DAPI in $10 \mathrm{ml} 2 \mathrm{xSSC}$-Tween) was pipetted on each microscope slide, incubated for two minutes and subsequently washed with deionized water. The slides were air-dried in a dark chamber, embedded with $50 \mu$ l antifade solution and covered with a cover slip. The slides were stored at $4^{\circ} \mathrm{C}$ and used for analysis. 


\subsection{Immunostaining}

Cultured cell lines were grown on microscope slides, fixed with $4 \%$ formaldehyde for 15 minutes at room temperature and washed with PBS. Tissue sections had to be deparaffinized and rehydrated before usage. The microscope slides carrying the tissue sections were washed two times with xylene for 10 minutes each, two times with $100 \%$ ethanol for five minutes each, two times with $95 \%$ ethanol for five minutes each and one time with $\mathrm{H}_{2} \mathrm{O}$ for five minutes. For antigen unmasking, the slides were placed in ten mM sodium citrate buffer $(\mathrm{pH}=6.0)$ which was heated to $95-99^{\circ} \mathrm{C}$ and maintained for ten minutes. The slides were cooled for 30 minutes in the buffer, rinsed three times with $\mathrm{H}_{2} \mathrm{O}$ for five minutes each and one time with PBS.

To block endogenous peroxidases (for immunohistochemistry), the slides were incubated with $3 \% \mathrm{H}_{2} \mathrm{O}_{2}$ for ten minutes and rinsed two times with PBS. Unspecific signals were blocked for 60 minutes with blocking buffer, containing serum of the animal in which the second antibody was produced. The primary antibody was diluted according to the manufacturer's instructions. The blocking solution was aspired and the tissue sections were incubated with the primary antibody over night at $4^{\circ} \mathrm{C}$, rinsed three times with PBS for five minutes each and incubated with the fluorochrome-conjugated secondary antibody, diluted in antibody dilution buffer, for one to two hours at room temperature in the dark. In case of immunofluorescence, the slides were washed with PBS and covered with Vectashield mounting medium. In case of immunohistochemistry the slides were washed with PBS and incubated in DAB solution until appropriate staining was visible. The reaction was stopped with PBS and the slides were washed with PBS three times for five minutes each. Counterstaining was performed using haematoxylin for ten seconds. The slides were washed with $\mathrm{H}_{2} \mathrm{O}$ for five minutes, with ethanol $(70 \%, 80 \%$ and $96 \%)$ for one minute each and covered with xylolbased mounting medium. The slides were stored at $4^{\circ} \mathrm{C}$ in the dark. 


\subsection{Tissue culture}

\subsubsection{Cultivation conditions}

Cells were cultured in a humidified incubator with $5 \% \mathrm{CO}_{2}$ at $37^{\circ} \mathrm{C}$. All work was performed in a class II laminar flow hood with sterile material. Medium was changed every second day and cells were passaged if they reached a confluence of about $80-90 \%$. For passaging of cells, medium was aspirated, cells were washed with PBS, incubated with Accutase for ten minutes at $37^{\circ} \mathrm{C}$, diluted with fresh medium and passaged to a new flask.

\subsubsection{Cryoconservation}

Cells were detached from the flask using accutase, diluted with medium and centrifuged for five minutes at $320 \mathrm{~g}$. The pellet was resuspended in one ml cryo medium containing 10\% DMSO and 50\% FCS and transferred to a cryo-vial which was immediately frozen at $-80^{\circ} \mathrm{C}$. For long-term storage the vial was transferred to liquid nitrogen.

For thawing of cells the vial was transferred to a water bath at $37^{\circ} \mathrm{C}$. At the point the cryo medium was almost completely liquid, it was rapidly transferred to five $\mathrm{ml}$ pre-warmed fresh culture medium. Cells were spun down for five minutes at $320 \mathrm{~g}$, resuspended in fresh culture medium and cultivated in the incubator.

\subsubsection{Cell isolation}

Tissues for cell isolation were either obtained from the TUM facility for livestock at Thalhausen or from the slaughterhouse. All animals used were pure German Landrace breed. For the first week, medium was changed every day and cells were cultured in medium containing penicillin, streptomycin and amphotericin B. Afterwards antibiotic-free medium was used. 


\subsubsection{Isolation of adipose tissue derived MSC}

For the isolation of adipose tissue derived MSCs (adMSCs) the neck of the slaughtered animal was cleaned with soap, thoroughly washed, all hairs were removed and the skin was sprayed with barricidal, a disinfectant. A piece of about $5 \times 5 \mathrm{~cm}$ was cut out of the neck fat and placed in PBS (with antibiotics) or $80 \%$ ethanol for transport. All equipment for cell isolation was cleaned with $80 \%$ ethanol. A piece of about $1.5 \times 1.5 \mathrm{~cm}$ was cut out in the middle of the fat tissue, washed with $80 \%$ ethanol and PBS for three times each and used for cell isolation. The tissue was cut into very small pieces and incubated with ten $\mathrm{ml}$ of collagenase I solution $(0.1 \%)$ for 30 minutes at $37^{\circ} \mathrm{C}$ on a magnetic stirrer. The solution was mixed with ten ml medium and cells were additionally separated through a $100 \mu \mathrm{m}$ filter. Cells were centrifuged for ten minutes at $320 \mathrm{~g}$, the supernatant was removed and the pellet resuspended in medium, which was distributed to four T-150 flasks.

\subsubsection{Isolation of bone marrow derived MSCs}

The bone ends of femur and tibia were cut off and bone marrow cells were flushed with a heparin solution (1000U heparin/ ml HBSS). The solution was loaded on a lymphocyte separation medium and centrifuged for ten minutes at 1000g. Cells from the gradient interphase were harvested, washed with $25 \mathrm{ml}$ HBSS and centrifuged for ten minutes at 600g. Subsequently they were resuspended in medium, plated one T-75 flask per bone and cultured.

\subsubsection{Isolation of kidney fibroblasts}

A piece of about $1 \times 1 \mathrm{~cm}$ was cut out of the kidney. The kidney-skin, blood vessels and fat were removed and the tissue was washed three times with $80 \%$ ethanol and PBS each. Mincing of the tissue was followed by several washing steps using PBS and collagenase type II $(10 \mathrm{mg} / \mathrm{ml})$ digestion for 30 minutes at $37^{\circ} \mathrm{C}$. Medium was added and cells were centrifuged for ten minutes at $320 \mathrm{~g}$. The cell pellet was resuspended in medium and plated on two T-150 flasks. 


\subsubsection{Isolation of aorta endothelial cells}

The porcine aorta was cut out of the heart tissue, cleaned properly and washed several times with PBS. After an incubation time of 45 minutes at $37^{\circ} \mathrm{C}$ with collagenase type II solution (ten $\mathrm{mg} / \mathrm{ml}$ ), the aorta was flushed with medium several times. Cells were collected and centrifuged for five minutes at $320 \mathrm{~g}$. The pellet was resuspended in medium and cells were cultured one T-25 flask.

\subsection{Transfection}

\subsubsection{Electroporation}

Cells were detached from the flask using accutase. $1 \times 10^{6}$ cells were centrifuged for five minutes at $320 \mathrm{~g}$ and resuspended in $800 \mu$ hypo-osmolar buffer. DNA was added and the suspension was pipetted to an electroporation cuvette (four $\mathrm{mm}$ ), incubated for five minutes at room temperature and pulsed at $1.2 \mathrm{kV}$ for 85 $\mu$ s. After five minutes incubation time, the suspension was diluted with one $\mathrm{ml}$ medium and transferred to a 6-well plate. The next day, cells were washed with PBS and medium was changed.

\subsubsection{Lipofection}

Cells were transfected using Lipofectamine 2000 at 40-70\% confluence. Medium was aspired, cells were washed with PBS and cultured in Opti-MEM. Per $10 \mathrm{~cm}$ dish, 5-15 $\mu$ g of DNA, dependent on the size of the constructs, were mixed with Opti-Mem (300 $\mu$ l total volume). Six $\mu$ l of Lipofectamine were mixed with $294 \mu \mathrm{l}$ Opti-Mem, incubated for five minutes at room temperature and added dropwise to the DNA solution. The solutions were mixed briefly and incubated for 20-25 minutes at room temperature. Subsequently the DNA-Lipofectamine solution was added dropwise directly on the cells. After six hours of incubation time in the incubator, MSC-medium was added and the cells were cultured over night. The next day, medium was changed. 


\subsection{Selection of stably transfected clones}

Selection was started 48 hours after transfection. AdMSCs were selected using Blasticidin S, G418 or Hygromycin at the determined optimal concentration. Medium was changed at least every second day. When single cell clones were visible, selection was stopped. Clones were picked using cloning rings and Accutase and further expanded.

\subsection{Isolation of mammalian genomic DNA and RNA}

RNA from mammalian cells was isolated using the High Pure RNA Isolation Kit. Genomic DNA and RNA from cells were isolated using the SurePrep RNA/DNA/Protein Purification Kit. Moreover, the SpeedMill PLUS homogenisator in combination with the innuSPEED kits was used for DNA and RNA isolation from tissues. For homogenisation two cycles of 30 seconds were chosen. All isolations were done according to the manufacturer's instructions.

To check RNA integrity three $\mu$ l of RNA were mixed with three $\mu \mathrm{l}$ of $2 x$ loading dye and heated for ten minutes at $70^{\circ} \mathrm{C}$, cooled on ice for five minutes and loaded on a 1\% TBE gel containing formamide. Strong bands of the $18 \mathrm{~S}$ and $28 \mathrm{~S}$ ribosomal RNA confirmed a successful RNA isolation.

\subsection{9 cDNA synthesis}

CDNA was produced using the SuperscriptIII Reverse Transcriptase kit according to the manufacturer's protocol.

\subsection{Quantitative real time PCR}

Detection of expression levels was conducted using the TaqMan Fast Universal PCR Master Mix together with 5' FAM/3' BHQ labeled probes (MWG Eurofins Operon; Ebersberg, Germany). Each sample was measured 3-fold using ten $\mu \mathrm{l}$ approaches and MicroAmp Fast Optical 96-Well Reaction Plates in combination with the 7500 Fast Real-Time PCR Cycler. 
Initial denaturation was performed for 30 seconds at $95^{\circ} \mathrm{C}$ followed by 45 cycles of denaturation for three seconds at $95^{\circ} \mathrm{C}$ and annealing/elongation for 30 seconds at $60^{\circ} \mathrm{C}$. As house-keeping gene Gapdh was chosen. The reference cell line for comparison of expression levels was the immortalized human MSC cell line SCP1. Expression levels were calculated according to equations 1-3.

\begin{tabular}{ll}
\hline$\Delta \mathrm{c}_{\mathrm{t}}=\mathrm{c}_{\mathrm{t}}($ target gene $)-\mathrm{c}_{\mathrm{t}}(\mathrm{Gapdh})$ & Equation 1 \\
\hline$\Delta \Delta \mathrm{c}_{\mathrm{t}}=\Delta \mathrm{c}_{\mathrm{t}}$ (clone) $-\Delta \mathrm{c}_{\mathrm{t}}(\mathrm{SCP} 1)$ & Equation 2 \\
\hline $\mathrm{x}$-fold expression level $=2^{-\Delta \Delta \mathrm{ct}}$ & Equation 3 \\
\hline
\end{tabular}

\subsection{Protein isolation of mammalian cells}

For protein isolation, about $70-80 \%$ confluent cells from two T-150 flasks were washed with PBS, detached using Accutase and spun down. The supernatant was removed and the pellet was resuspended in $1 / 3$ of the recommended volume of CytoBuster Protein Extraction Reagent (containing proteinase inhibitor cocktail). Cells were incubated for five minutes at room temperature and for 30 minutes at $-80^{\circ} \mathrm{C}$. Cell debris was spun down for five minutes at $16000 \mathrm{~g}$ and $4^{\circ} \mathrm{C}$. Aliquots from the supernatant were made and stored at $-80^{\circ} \mathrm{C}$.

Additionally protein isolation was carried out using the Mammalian Cell Lysis Kit according to the manufacturer's instructions.

\subsection{Western Blot}

Protein concentration was determined by mixing five $\mu \mathrm{l}$ of protein extract with $995 \mu$ l Protein Quantification Reagent. The solution was incubated for five minutes at room temperature and absorption was measured three-fold per sample at $595 \mathrm{~nm}$. Samples were mixed with 4x Lämmli buffer, incubated for five minutes at $95^{\circ} \mathrm{C}$ and spun down. Ten to $40 \mu \mathrm{g}$ of protein per lane were separated by a $12 \%$ polyacrylamide gel (containing a $5 \%$ collection gel). The gels were run for 20 minutes at $100 \mathrm{~V}$ and for 45 minutes at $200 \mathrm{~V}$. A PVDF membrane was cut out, incubated for one minute in $100 \%$ ethanol and washed with semi-dry blotting buffer. Two filter papers per blot were laid in semi-dry blotting buffer. 
The semi-dry blotting chamber was assembled according to the manufacturer's instructions, air bubbles were removed and each gel was run at ten $\mathrm{V}$ for 30 minutes. A successful transfer was confirmed by Ponceau $S$ staining. The membrane was washed three times for one minute and three times for 15 minutes with 1x TBST. Blocking was performed for one hour at room temperature with $1 \mathrm{x}$ TBST containing 5\% BSA or 5\% skim milk powder. The primary antibody was diluted in $1 \mathrm{x}$ TBST blocking solution according to the manufacturer's instructions and incubated over night at $4^{\circ} \mathrm{C}$. Subsequently the membrane was washed three times for one minute and three times for 15 minutes with $1 \mathrm{x}$ TBST. The secondary antibody was diluted according to the manufacturer's instructions in 1x TBST blocking solution and the membrane was incubated for one hour at $4^{\circ} \mathrm{C}$. The membrane was washed as before and incubated with staining solution (ECL reagent A and B). Detection of signals was carried out by exposure of the membrane to an X-ray film for one minute up to 60 minutes.

\subsection{ZFN-mediated GGTA1 gene inactivation}

A pair of plasmids encoding a zinc-finger nuclease (ZFN) targeted to GGTA1 exon eight was transfected into PKDNF from piglet 1706 by electroporation by standard methods. $\alpha$ Gal-negative cells were enriched by counter-selection using streptavidin-coated magnetic beads and biotin-conjugated isolectin B4 in a magnetic field (Hauschild et al., 2011). GGTA1 gene editing was analysed by allele-specific sequencing of cloned PCR fragments amplified across the ZFN target site.

\subsection{Immunofluorescence staining and flow cytometry}

Cell surface molecules were detected by flow cytometry. $0.1-0.5 \times 10^{6}$ cells were incubated with one of the following antibodies: anti-human CD46-PE, antihuman CD55-bio, anti-human CD59-PE, anti-porcine CD62e FITC, anti-porcine MHC class-II. 
Binding of biotinylated primary antibodies was visualized using streptavidin-PE and binding of unlabeled primary reagents visualized using goat anti-mouse IgGFITC secondary antibody. The presence or absence of $\alpha 1,3-G a l$ epitopes was determined using a tetrameric isolectin B4-FITC. Cells were analysed on a FACSCalibur flow cytometer equipped with CellQuest Pro Software. Dead cells were excluded from the analysis.

\subsection{Assay for complement-mediated lysis of porcine fibroblasts}

51-Chromium release assays were performed as described (Hauschild et al., 2011). Fibroblasts were labeled with $100 \mu \mathrm{Ci}$ sodium $\left[{ }^{51} \mathrm{Cr}\right]$-chromate. $1 \times 10^{4}$ cells/well were incubated with $2.5 \%, 5 \%, 10 \%$ or $20 \%$ pooled complementpreserved normal human serum. After four hours, $25 \mu \mathrm{L}$ cell supernatant were removed and radioactivity measured in a Microbeta scintillation counter. The mean cpm obtained in triplicate cultures was used for all calculations. Spontaneous ${ }^{51} \mathrm{Cr}$ release was determined by incubating cells with medium alone, and maximum release determined by incubating in 2\% Triton X-100. Specific lysis was calculated as: \% specific lysis $=\left(\right.$ experimental ${ }^{51} \mathrm{Cr}$ release spontaneous ${ }^{51} \mathrm{Cr}$ release) / (maximum ${ }^{51} \mathrm{Cr}$ release - spontaneous ${ }^{51} \mathrm{Cr}$ release) $\times 100$.

\subsection{Cytokine-induced upregulation of E-selectin and MHC class-II}

PAECs (passages two-five) were stimulated with $50 \mathrm{ng} / \mathrm{mL}$ human $\mathrm{TNF} \alpha$ or eight $\mathrm{ng} / \mathrm{mL}$ or $50 \mathrm{ng} / \mathrm{mL}$ porcine IFN $\gamma$. After two days TNF $\alpha$ treatment, cells were analysed for E-selectin (CD62e) expression. After three days IFN $\gamma$ treatment, porcine MHC class-II upregulation was determined via flow cytometry. CD62e or MHC class-II upregulation was calculated as fold-increase of mean fluorescence intensity normalized to background staining and relative to untreated cells. 


\section{Results}

Multi-transgenic pigs for xenotransplantation have so far been derived by breeding of single or double transgenic animals. Each time transgenic animals are bred, the transgenes segregate and large herds are required to obtain multitransgenic animals expressing all desired genes. Unfortunately, most bred animals are of little experimental value. Therefore, new strategies are required also with view of the 3Rs (reduce, refine, replace). Generation of improved constructs (refine) by combining several transgenes on one expression vector enables a better pre-selection of cell clones in cell culture (reduce). Moreover, one single genomic integration site of all vectors causes a drastic reduction of the animal number needed (reduce).

Bacterial artificial chromosome (BAC) constructs themselves can be modified through recombineering to reduce the size or to add additional xenogenes. The following sections describe how the BAC carrying the genomic sequence of CD55 was reduced in size and subsequently modified by the addition of the coagulation regulatory gene human thrombomodulin (TM) and the T-cell regulatory gene LEA29Y or the anti-inflammatory and anti-apoptotic genes A20 and H01. These multi-gene BACs reduce the risk of various integration sites. Several of the CD55 BACs were cotransfected with CD46 BAC and CD59 PAC constructs to generate human artificial chromosomes (HAC) or multi-transgenic cell clones.

As human artificial chromosomes can carry whole genomic loci and all complement regulatory genes, the formation of a human artificial chromosome in human HT1080 cells was to be tested by cotransfection experiments of the CD46 BAC, CD55 BAC, CD59 PAC and HAC constructs. The technique of microcell mediated chromosome transfer was subsequently optimized to enable the transfer of human artificial chromosomes between human and porcine cells. Cotransfection experiments of the CD46 BAC, CD55 BAC and CD59 PAC constructs were performed to obtain cell clones expressing various xenograftprotective genes at once. Clones with the highest expression levels were used for somatic cell nuclear transfer. 
Two transgenic founder animals were obtained. One animal expressing CD46 and CD55 and a multi-transgenic animal expressing CD46, CD55, CD59, H01 and A20.

Other members of the Chair of Livestock Biotechnology showed that the porcine ROSA26 locus can be gene targeted with high efficiency and supports ubiquitous transgene expression. An existing heme oxygenase 1 cDNA construct was to be used for gene-placement. Correct targeted clones were used for somatic cell nuclear transfer and a transgenic animal was analyzed for the expression of heme oxygenase 1 . A retargeting construct of ROSA26 was prepared to place the CD55 minigene adjacent to the heme oxygenase 1 transgene, thus allowing the combination of several xeno-protective genes at a single predetermined genomic locus.

\subsection{Generation of xeno-gene expression vectors}

To enable the work outlined above, a number of DNA constructs had to be generated including selection cassettes, genomic and cDNA constructs as well as minigenes for the expression of xeno-protective genes.

\subsubsection{Resistance cassettes for pro- and eukaryotic cells}

It was necessary to prepare different resistance cassettes to enable selection of bacteria after recombineering was carried out or for the selection of transfected porcine cells. The CMV driven spectinomycin gene was to be used for the selection of bacteria, the SV40/EM7 driven BS cassette for the selection of transfected porcine cells and the PGK/EM7 Neo cassette was to be used to select either bacteria using kanamycin or porcine cells using G418.

Two constructs were produced, consisting either of the vector PL452 with the spectinomycin cassette (Fig. 8C) or the vector PL452inf with a BS cassette (Fig. 9C). These cassettes were subsequently inserted into plasmids carrying xenogenes. 


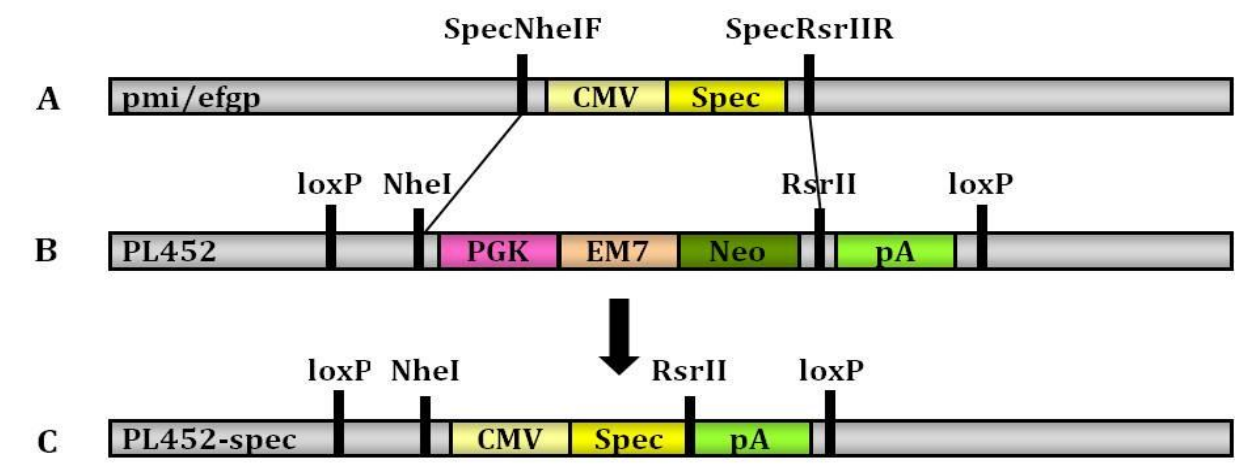

Figure 8. Schematic diagram for cloning of the plasmid PL452-spec. A: PCR amplification of the plasmid pmi/egfp using the primer combination SpecNheIF-SpecRsrIIR resulted in a DNA fragment containing the spectinomycin resistance cassette flanked by NheI/RsrII restriction sites, enabling cloning between the NheI/RsrII restriction sites of PL452 (B), resulting in the plasmid PL452-spec (C).

A

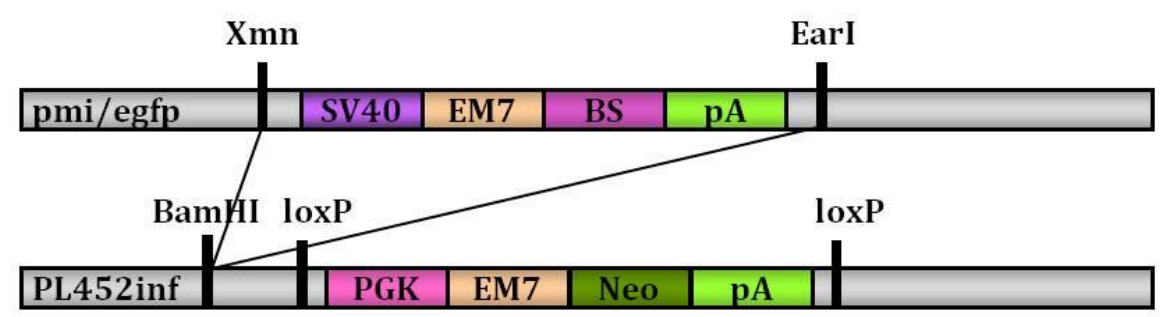

C \begin{tabular}{|l|l|l|l|l|l|l|l|l|l|}
\hline PL452inf-BSinf & SV40 & EM7 & BS & pA & & & & Neo & \\
\hline
\end{tabular}

Figure 9. Schematic diagram for cloning of the plasmid PL452inf-BSinf. A: Excision of the SV40/EM7 BS cassette out of the plasmid pmi/egfp using XmnI and EarI and blunt end insertion into the BamHI digested vector PL452inf (B), resulting in the plasmid PL452inf-BSinf (C).

\subsubsection{Genomic constructs}

\subsubsection{CD46 and CD59 constructs}

Genomic constructs of the complement regulatory genes CD46, CD55 and CD59 were chosen to enable expression of human complement regulatory genes in porcine cells and transgenic animals suitable for xenotransplantation. These genomic constructs express the full range of membrane bound and soluble protein isoforms. 
However, the commercially available constructs from Source Bioscience were very large and had to be modified to delete flanking regions and regions not necessary for gene function or regulation, resulting in much smaller constructs that are easier to handle. The BAC construct carrying the genomic sequence of CD46 and the PAC construct carrying the genomic sequence of CD59 were modified by various restriction digests and recombineering steps. Figure ten summarizes the modifications that were performed by M. Edlinger, S. Christan and S. Kraner-Scheiber.

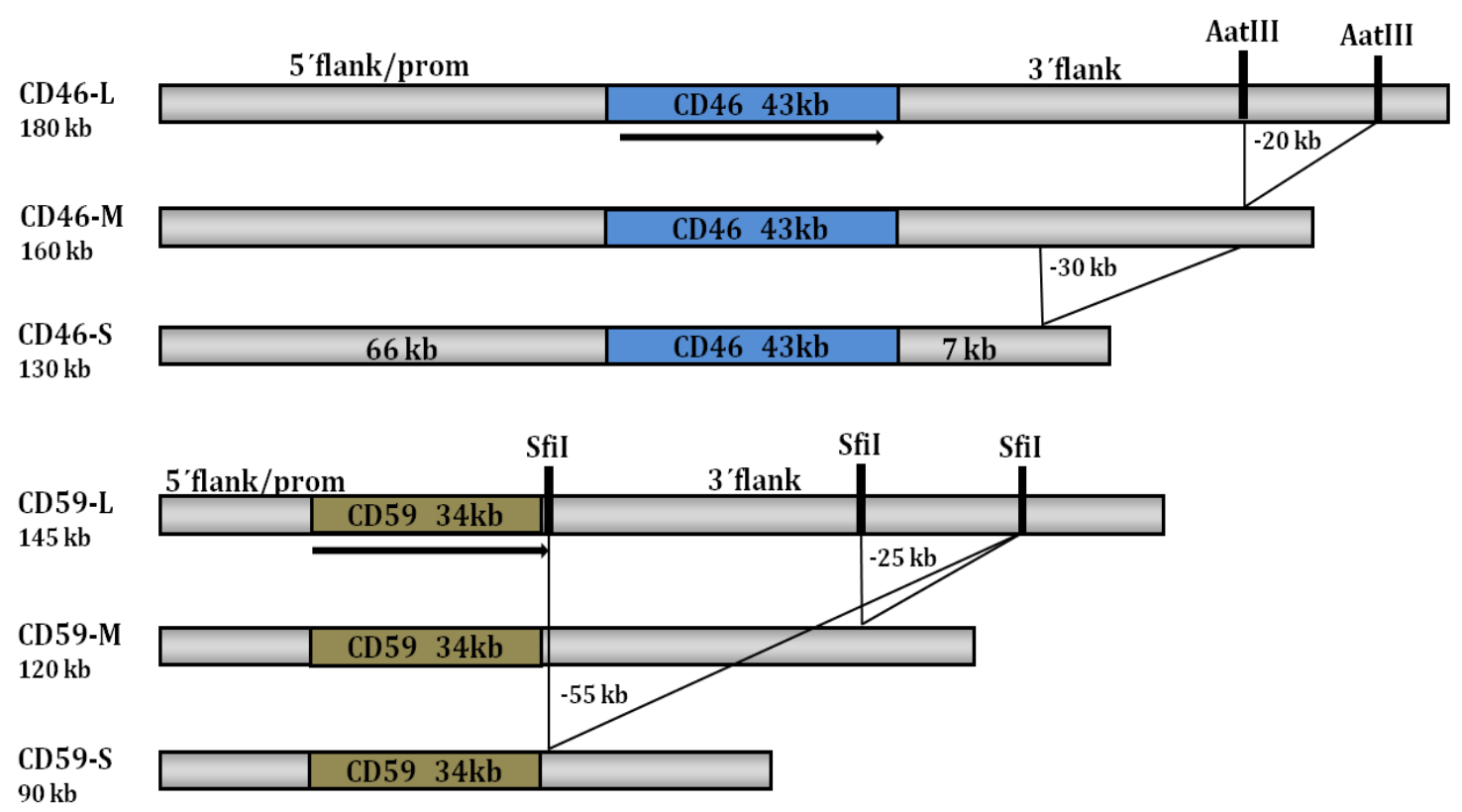

Figure 10. Schematic diagram of the structure and modifications of the CD46 BAC and CD59 PAC constructs. The original CD46 BAC (L-construct) purchased from Source Bioscience was reduced by deletion of a $20 \mathrm{~kb}$ AatI fragment resulting in the CD46-M construct. This construct was reduced further by $30 \mathrm{~kb}$ by a recombineering step resulting in the CD46-S construct. The original CD59 PAC construct (L-construct) was also purchased from Source Bioscience. This construct was Sfil digested resulting in the CD59-M construct by a $25 \mathrm{~kb}$ deletion and the CD59-S construct by a $55 \mathrm{~kb}$ deletion. Resistance cassettes and the BAC- or PAC-backbone are not shown. 


\subsubsection{CD55 and CD55 multi-transgene constructs}

The size of the BAC construct carrying the genomic sequence of CD55 was reduced in three steps. The resulting smallest CD55 construct (CD55-XS) was subsequently used to place additional xenograft-protective genes such as human thrombomodulin or the T-cell regulatory gene LEA29Y on the CD55 BAC construct.

\subsection{CD55 constructs}

The size of the original CD55 BAC construct (Source Bioscience) was reduced in three consecutive steps by digestion and recombineering as outlined in figure 11.

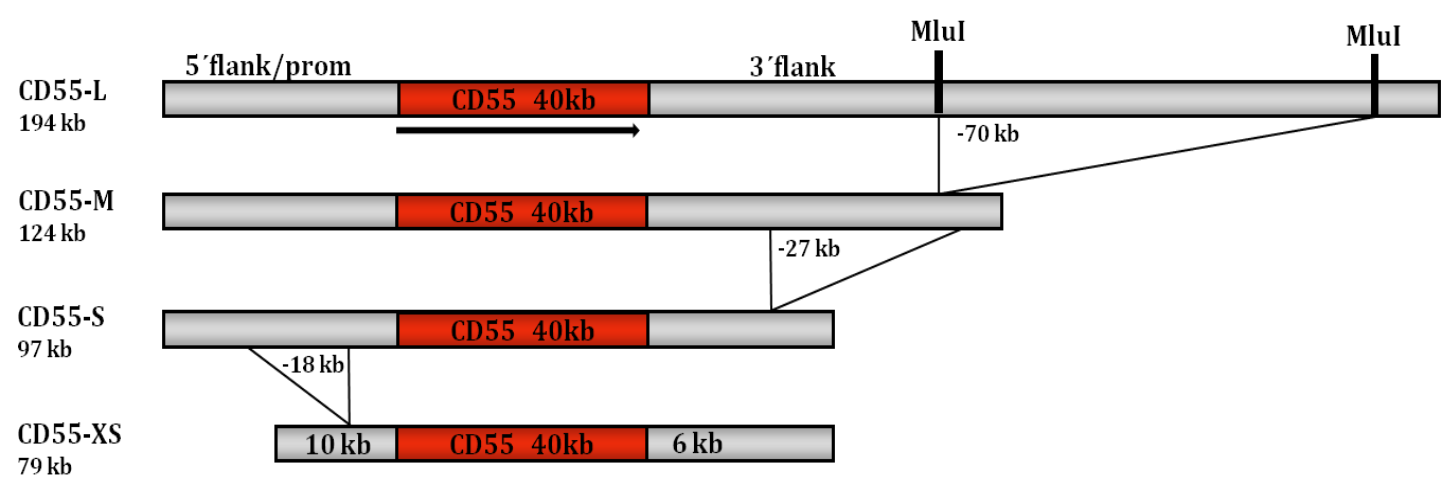

Figure 11. Schematic diagram showing the various modifications of the CD55 BAC construct. The size of the original CD55 BAC (L-construct) was reduced by deleting $70 \mathrm{~kb}$ in the $3^{\prime}$ flanking region (MluI digest) resulting in the CD55-M construct. This construct was further reduced in size by a recombineering step deleting further $27 \mathrm{~kb}$ in the $3^{\prime}$ flanking region resulting in the CD55-S construct. This construct should now be further reduced in size by an $18 \mathrm{~kb}$ deletion in the 5' flanking/promoter region. Resistance cassettes and the BAC-backbone are not shown.

Reducing the size of CD55 BAC constructs from $194 \mathrm{~kb}$ to $79 \mathrm{~kb}$ (CD55-XS) had several advantages such as better handling of the construct, easier transfections and was the prerequisite for the generation of multi-transgene BAC constructs (the CD55-A20-H01 and the CD55-TM-LEA constructs). 
The CD55-XS construct, compared to the CD55-S construct, has an $18 \mathrm{~kb}$ deletion in the 5' CD55 flanking region. The remaining 5' flanking and promoter region of the XS-construct has a size of $10 \mathrm{~kb}$. The general question was, whether this deletion is possible without affecting any regulatory CD55 elements and the endogenous CD55 promoter activity. A floxed neomycin resistance cassette and a BS resistance cassette were also inserted into the CD55 construct by homologous recombination to improve selection possibilities. Figure 12 shows the cloning steps for the recombineering vector. Recombineering was conducted in SW106 cells containing the CD55-S construct (Figure 12).

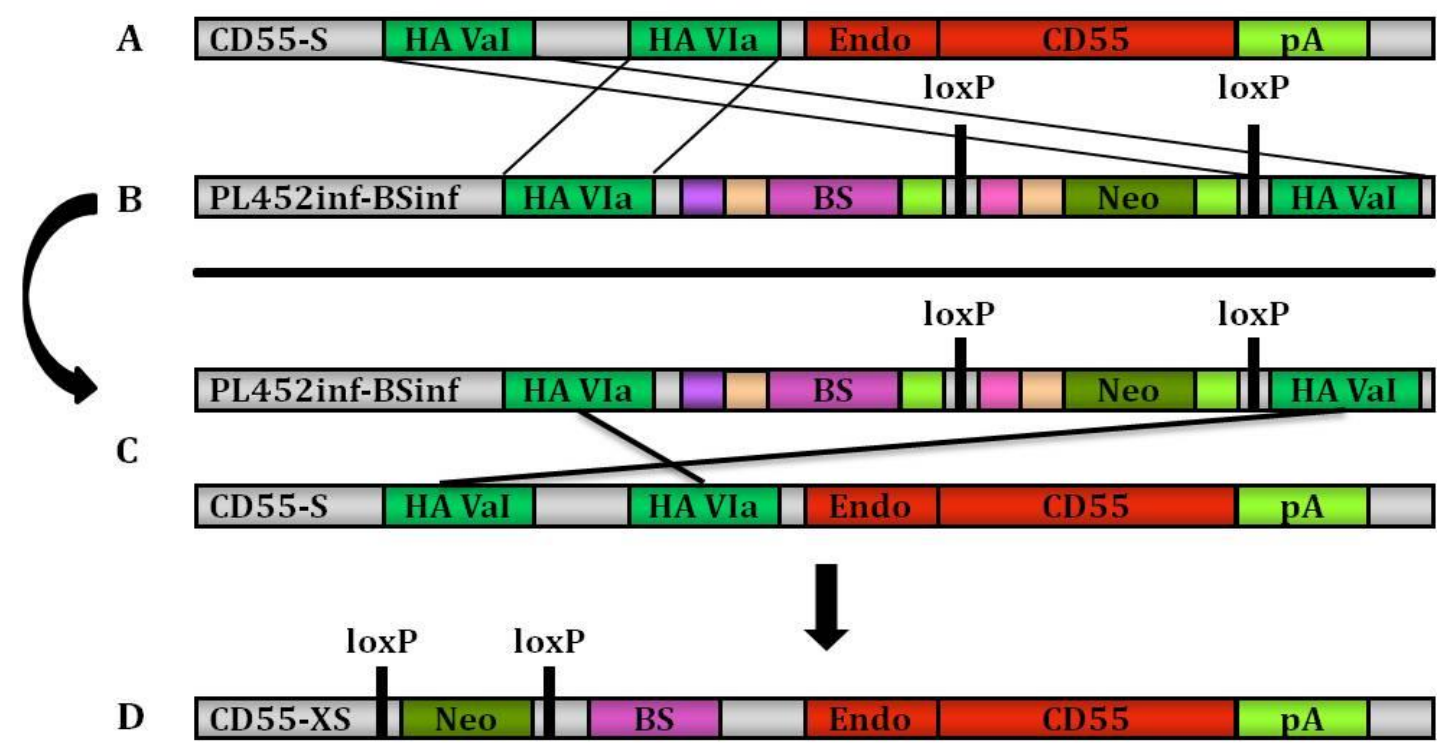

Figure 12. Schematic diagram of the cloning steps for the CD55-XS construct carrying a BS and neomycin resistance cassette. A: Amplification of homologous sequences (HA) Va1 (Primer: CD55 Va1F/R) and VIa (Primer: CD55VIa F/R) using the CD55-S construct as template. B: Cloning of homology arm Va1 into PL452inf-BSinf (ApaI, KpnI digested) and cloning of homology arm VIa into PL452inf-BSinfVa1 (NotI, SacII digested). Pink or beige colored small boxes represent the PGK or EM7 promoters for Neo. Purple or beige colored small boxes represent the SV40 or EM7 promoters for BS. These boxes are not shown in figure D due to space limitations. C: Homologous recombination between PL452inf-BSinfVa1VIa and the CD55-S construct. D: CD55-XS construct containing an $18 \mathrm{~kb}$ deletion in the $5^{\prime}$ flanking region and a Neo/BS resistance cassette for selection. 
Transfection of the CD55-S and CD55-XS constructs into porcine MSCs confirmed that the $10 \mathrm{~kb} 5^{\prime}$ flanking and promoter sequence was completely functional and gives high expression values (as shown later in figure 15). The $18 \mathrm{~kb}$ deletion in the 5' flanking region does not affect promoter activity or any CD55 regulatory elements.

\subsection{CD55 multi-transgene constructs}

After verifying RNA expression of the CD55-XS construct, the next steps were to place additional xenogenes on the CD55 BAC. Combination of transgenes reduces the number of integration sites in the host genome and thus reduces the probability of segregation of xenogenes during breeding of the resulting animals.

\subsection{The CD55-XS-TM-LEA construct}

Thrombomodulin which inhibits blood coagulation and thus could protect against acute vascular rejection was placed in the 3' flanking region of the CD55$\mathrm{S}$ construct. The next step was to reduce the size of the CD55-S-TM construct and to insert LEA29Y which inhibits T-cell activation, simultaneously in the 5' flanking region of CD55. For this purpose, LEA29Y was cloned into the recombineering vector used to generate the CD55-XS construct. Figure 13 and 14 show an overview of the different cloning and recombineering steps. 


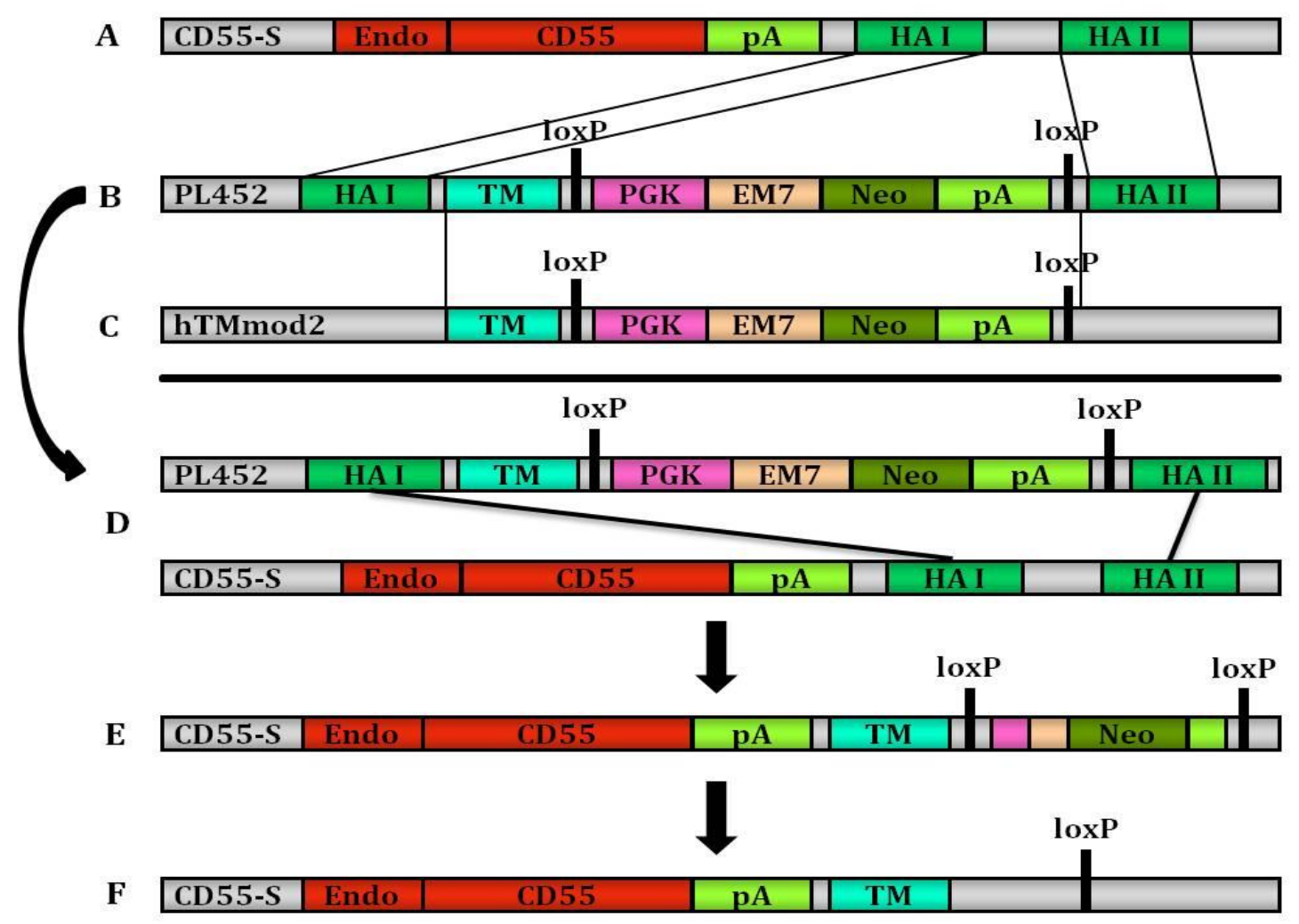

Figure 13. Schematic diagram of the cloning steps for the CD55-S-TM construct. A: Amplification of homologous sequences (HA) HAI (primer: LeaHAIFKpnI-LeaHAIRApaI) and HAII (primer: LeaHAIIFNotI-LeaHAIIRSacII) using the CD55-S construct as template. B: Cloning of HAI into PL452 (KpnI, ApaI digested) and HAII into PL452HAI (NotI, SacII digested). C: Cloning of a human TM and a PGK-EM7-Neo-pA cassette cut out of the plasmid hTMmod2 (Xhol digested) into PL452HAIHAII (Xhol digested). D: Recombineering of PL452HAIHAIITM with the CD55-S construct. E: CD55-S-TM construct with floxed neomycin resistance cassette. F: Floxing of the neomycin resistance cassette delivering the CD55-S-TM construct for the addition of LEA29Y. 
A

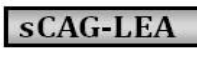

$1 \mathrm{D}$ Neo

B PL452inf-BSinf 1 HA VIa

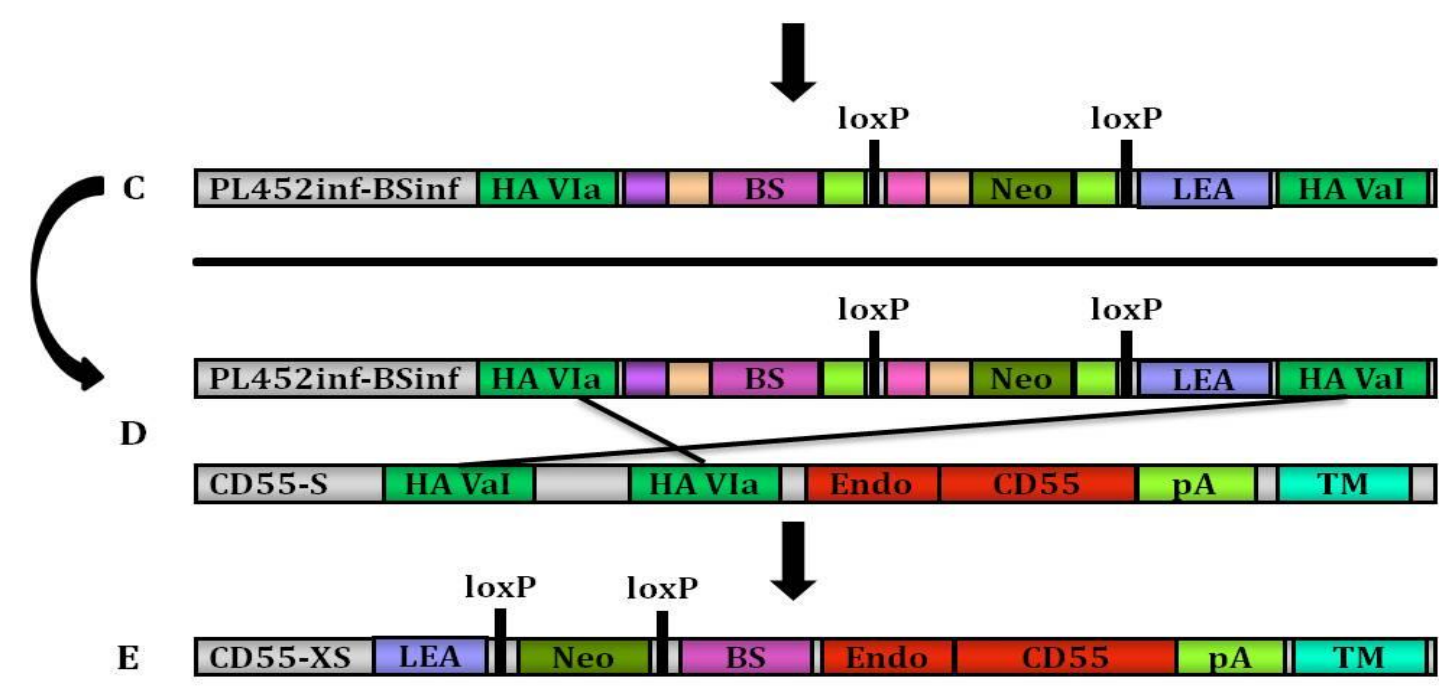

Figure 14. Schematic diagram of the cloning steps for the CD55-XS-TM-LEA construct: A: Plasmid sCAG-LEA. B: Cloning of LEA29Y into PL452inf-BSinf containing homology arm Va1 and VIa. For this step, the plasmid sCAG-LEA was AscI and EcoRV digested and the CAG-LEA cassette was cloned into PL452inf-BSinf (ApaI digested). Pink or beige colored small boxes represent the PGK or EM7 promoters for Neo. Purple or beige colored small boxes represent the SV40 or EM7 promoters for BS. These boxes are not shown in figure E due to space limitations. C: Plasmid PL452 containing homology arms Va1, VIa and LEA29Y. D: Recombineering of PL452infBSinfHAVa1HAVIa-LEA with the CD55-S-TM construct. During this step the size of the CD55-STM construct was reduced and TM was added. E: CD55-XS-TM-LEA construct, containing a floxed Neo cassette and BS.

Pulsed-field gel electrophoresis (PFGE) was used to verify the sizes of the different BAC constructs after each cloning step. AdMSC were stably transfected using the CD55-XS-TM-LEA construct. Figure 15 shows a RT-PCR result, verifying the expression of CD55, TM and LEA29Y. 


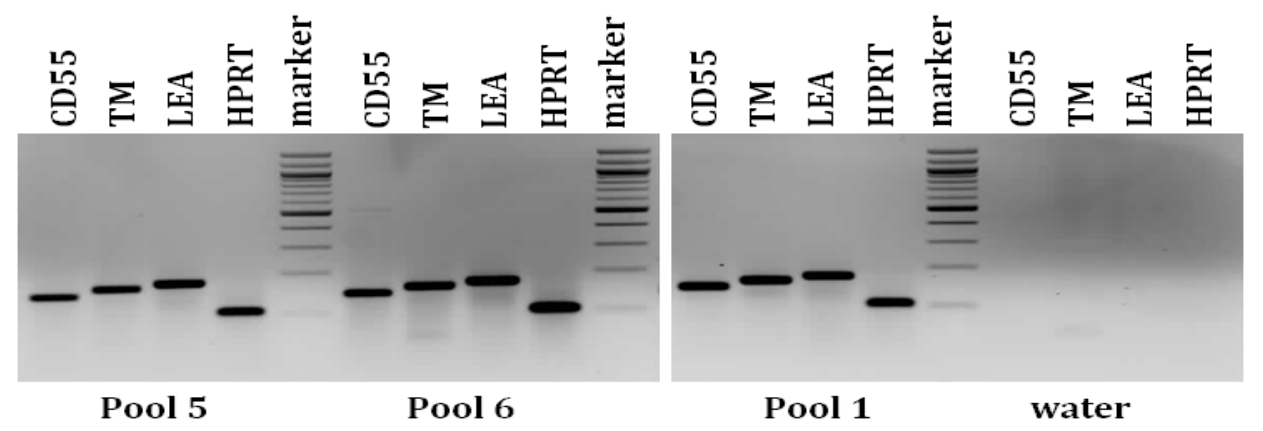

Figure 15. RT-PCR analysis of different adMSCs pools transfected with the CD55-XS-TM-LEA construct. Primer combinations used for detection: CD55: (Primer CD55 qPCRF2/R2) 142 bp; TM: (Primer TMncF/R) 153 bp; LEA29Y: (Primer LeaF/LeaExpr2R) 171 bp; HPRT: (Primer poHPRTF1/R1) 104 bp. Marker = 100 bp marker.

\subsection{The CAG-CD55-A20-H01 construct}

In parallel to the construction of the CD55-XS-TM-LEA construct, a CAG-driven CD55-XS construct was cloned, containing the anti-apoptotic and antiinflammatory genes A20 and HO1 (M. Edlinger and M. Herrmann). Figure 16 summarizes the different cloning steps that were performed for the construction of the CAG-CD55-A20-H01 construct. 


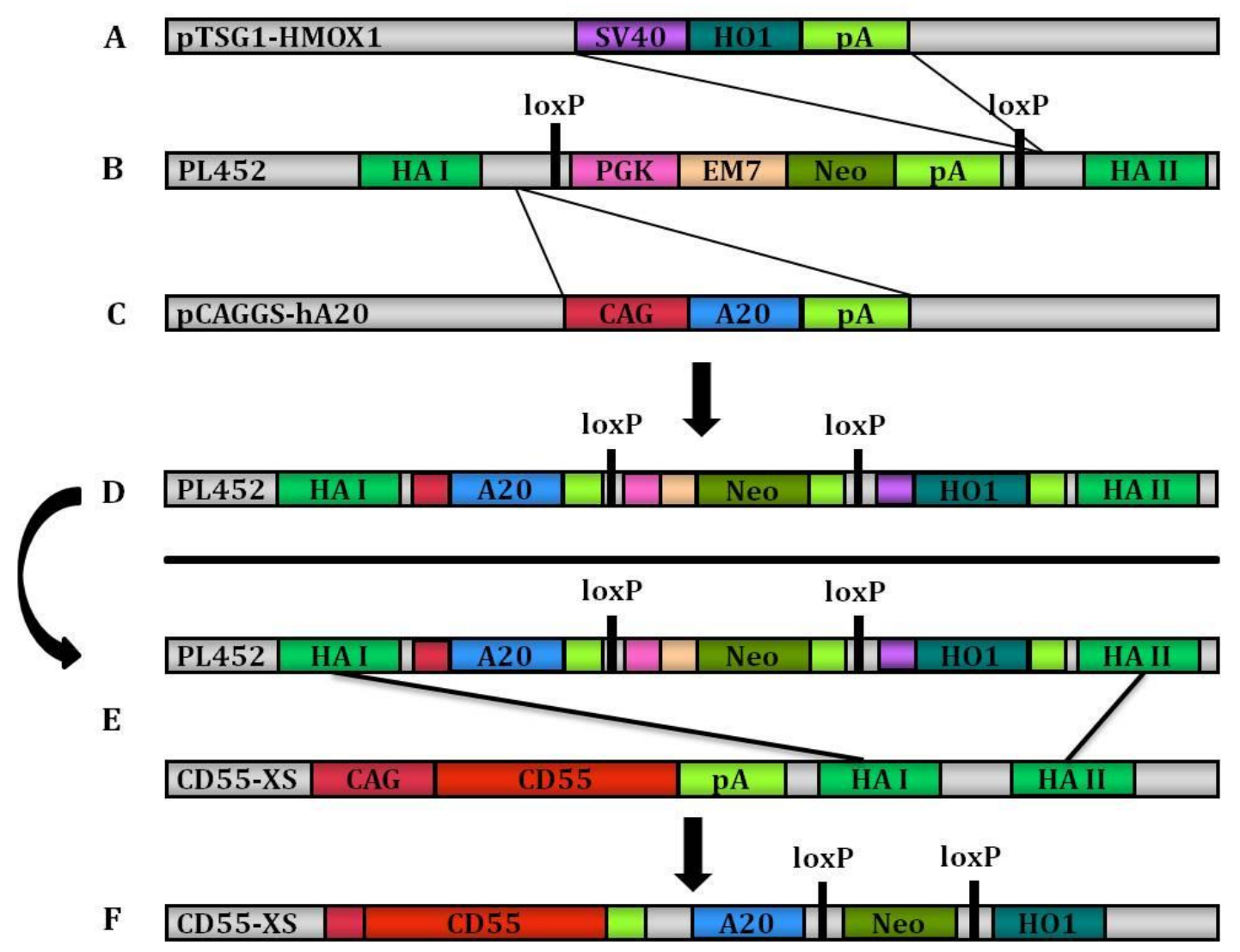

Figure 16. Schematic diagram of the different cloning steps necessary for the construction of the CAG-CD55-A20-HO1 construct. A: The SV40-H01-pA cassette was cut out of the plasmid pTSG1HMOX1 (BglII, EagI digested). B: Cloning of the SV40-H01-pA cassette and the CAG-A20-pA cassette into the plasmid PL452 (BamHI digested) carrying homology arms HAI and HAII. C. The CAG-A20-pA cassette was cut out of the plasmid pCAGGS-hA20 (DrdI, AccI digested). D: Plasmid PL452 carrying homology arms HAI, HAII, A20, HO1 and a floxed neomycin resistance cassette. Pink or beige colored small boxes represent the PGK or EM7 promoters for Neo. Red colored boxes represent the CAG promoter. Purple colored boxes represent the SV40 promoter. These boxes are not shown in figure $\mathrm{F}$ due to space limitations. E: This plasmid was used for a recombineering step with the plasmid CAG-CD55-XS. F: Final plasmid CAG-CD55-XS carrying an additional CAG-A20 and a SV40-H01 gene.

\subsubsection{Verification of correct CD55 splicing of BAC constructs}

The CD55 BAC construct was chosen for different modifications mentioned above. The advantage of genomic constructs is that they resemble the endogenous gene and should express all RNA splice variants, unlike cDNAs or minigenes which are generally based on a single RNA species. 
In the case of CD55, this is important because a range of RNA splice variants encoding membrane-bound and soluble isoforms are necessary for full biological activity. Functional splicing of the human CD55 gene in porcine cells had to be examined to ensure that the various modifications of the CD55 BAC construct did not alter the splicing pattern.

The CD55 gene has in total 13 splicing variants. Nine are translated into functional protein and four are not translated (www.ensembl.org). The most important splicing variants in humans are the GPI anchored CD55 (gDAF) and the soluble CD55 (sDAF). The gDAF protein is composed of four N-terminal complement control protein domains (CCP), a serine, threonine and proline (STP)-rich domain and a C-terminal GPI-anchored domain (Coyne et al., 1992). It is expressed on the membranes of all blood cells and almost all cell types in close contact with plasma component proteins. The expression levels of gDAF are much higher than those of sDAF, which is present in body fluids and the extracellular matrix (Medof et al., 1987). The sDAF molecule is formed by an alternative splicing and lacks the GPI-anchor (Caras et al., 1989).

To verify the correct splicing patterns of the different CD55 BAC constructs, the gDAF, sDAF as well as additional splicing variants were amplified by RT-PCR (Fig. 17).

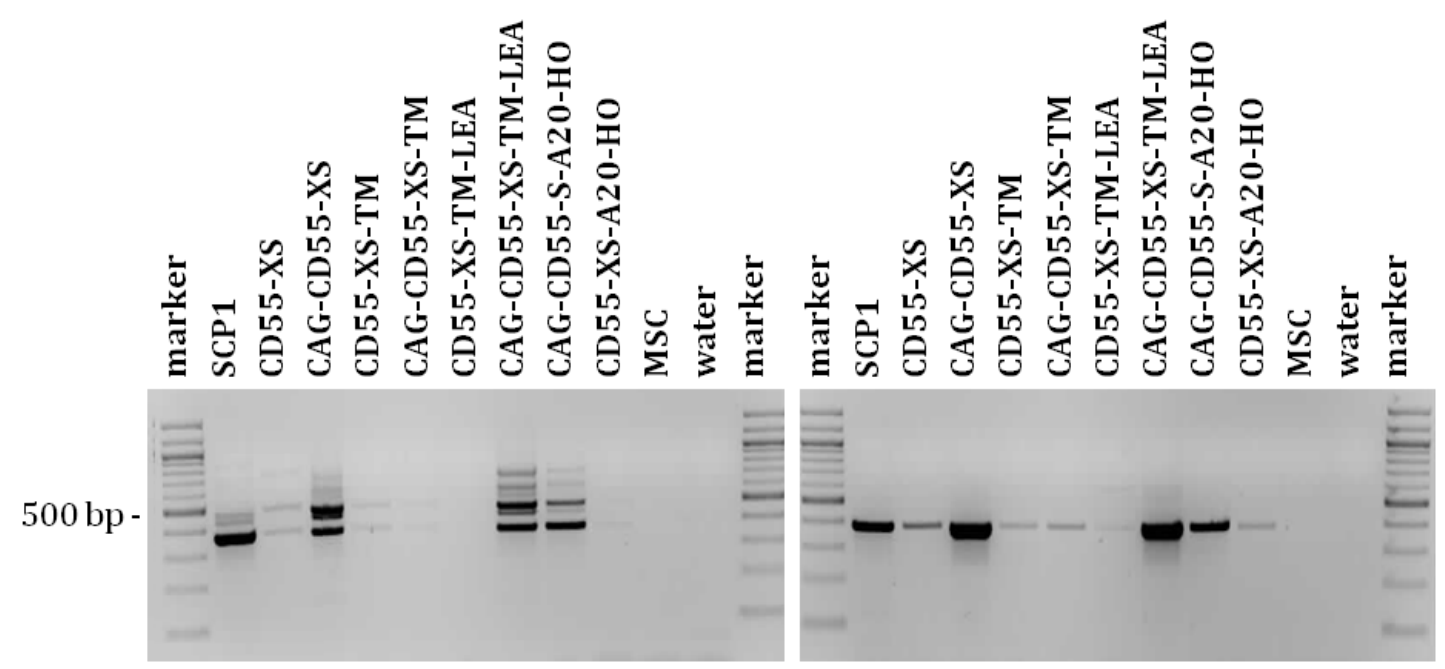

Figure 17. RT-PCR analysis of different CD55 splicing variants. Left: Primer combination DAF F1/R1 showing several CD55 splicing variants. Strongest bands represent RNA fragments encoding the membrane bound isoform ( $370 \mathrm{bp}$ ) and the soluble isoform (480 bp). Right: Verification of the soluble CD55 isoform using the primer combinations DAF F1/ sDAFR (380 bp). 


\subsubsection{Minigenes}

The size of genomic BAC or PAC constructs is not suitable for targeting approaches. To enable gene placement using the complement regulatory genes CD46 and CD55 or coagulation regulatory genes such as CD39, smaller minigene or cDNA constructs were prepared. The CD55 BAC construct was used for several modifications and transfections as already mentioned. However, the genomic CD55 BAC construct contains a $20 \mathrm{~kb}$ or $10 \mathrm{~kb}$ endogenous promoter sequence and the full length genomic CD55 sequence. The total size is $97 \mathrm{~kb}$ for the S-construct or $79 \mathrm{~kb}$ for XS-construct. Although genomic constructs include all biological splicing variants and almost all regulatory elements, the large size has some disadvantages. For a precise gene placement approach, the size of the construct is decisive.

A minigene differs from a cDNA construct in that it contains an intron to increase expression and reduce gene silencing. For gene placement approaches and for the generation of multi-xeno-gene constructs, a CAG-CD55 minigene, a CD46 minigene with an endogenous promoter and a CAG-CD39 cDNA construct were produced.

\subsubsection{CAG-CD55 minigene}

As several genomic CD55 BAC constructs were already prepared, the next aim was to clone a CD55 minigene which could be used for gene placement approaches. Figure 18 describes cloning of the CAG-CD55 minigene construct and shows its final structure. The size of the construct is $3.7 \mathrm{~kb}$ for the CD55 minigene and $6.5 \mathrm{~kb}$ for the CD55 minigene together with the Hygromycin resistance gene for selection. 


\begin{tabular}{|l|l|l|l|l|l|l|l|}
\hline \multicolumn{1}{l|}{ HindIII } \\
\hline pcDNA3.1-hygro(+) & CAG & Exon 1 & & Exon 2 & cDNA & pA & \\
\hline
\end{tabular}

Figure 18. The CAG-CD55 minigene consisting of the CAG promoter, exon 1, intron 1 and exon 2 up to the HindIII restriction site of the genomic CD55 sequence and the CD55 cDNA sequence ranging from the HindIII restriction site of exon 2 to the last exon of CD55. The cDNA part of the CD55 minigene, ranging from the HindIII restriction site of Exon 2 to the last exon of CD55, was amplified out of the cDNA, prepared from human MSC cell line SCP1, using the primer combinations CD55 Mini For2/CD55 cDNA ApaIrev, delivering a 958 bp fragment. The CD55 cDNA as well as the pcDNA3.1-hygro(+) vector were HindIII and ApaI digested and ligated. The genomic part of the CD55 minigene including exon 1, intron 1 and exon 2 up to the HindIII restriction site was amplified out of the CD55-S-CAG-HO-A20 construct using the primer combination CD55 Mini 5UTRMlu/CD55 Mini N2R, delivering a fragment of $828 \mathrm{bp}$. The pcDNA vector containing the CD55 cDNA as well as the genomic PCR fragment were HindIII and MluI digested and ligated. The CAG promoter was cut out of the plasmid pJet1.2-pCAG by a BglII restriction digest and cloned into the BglII restriction site of the pcDNA plasmid with the genomic and cDNA sequence of CD55. Although the pcDNA plasmid already contains a BGH-pA signal, an additional BGH-pA signal with several unique restriction sites at the end was ampilifed out of the vector PL452 using the primer combination PL452 4R/3shF. This PCR fragment was cloned into the PmeI restriction site of the pcDNA-CD55 minigene. This step was necessary to cut the whole promoter-CD55 genomic/cDNA sequence together with the polyA sequence out of the pcDNA plasmid without any unnecessary sequences.

AdMSC (Isolation number 0708) were transfected. Hygromycin resistant cell clones were picked and RNA was isolated. Expression was measured using Q-RTPCR. Subsequently, expression values were calculated and revealed very high expression levels of the CAG-CD55 minigene (Fig. 19). The highest expressing clones reached more than 100 fold higher expression levels of CD55 compared to human MSCs. 


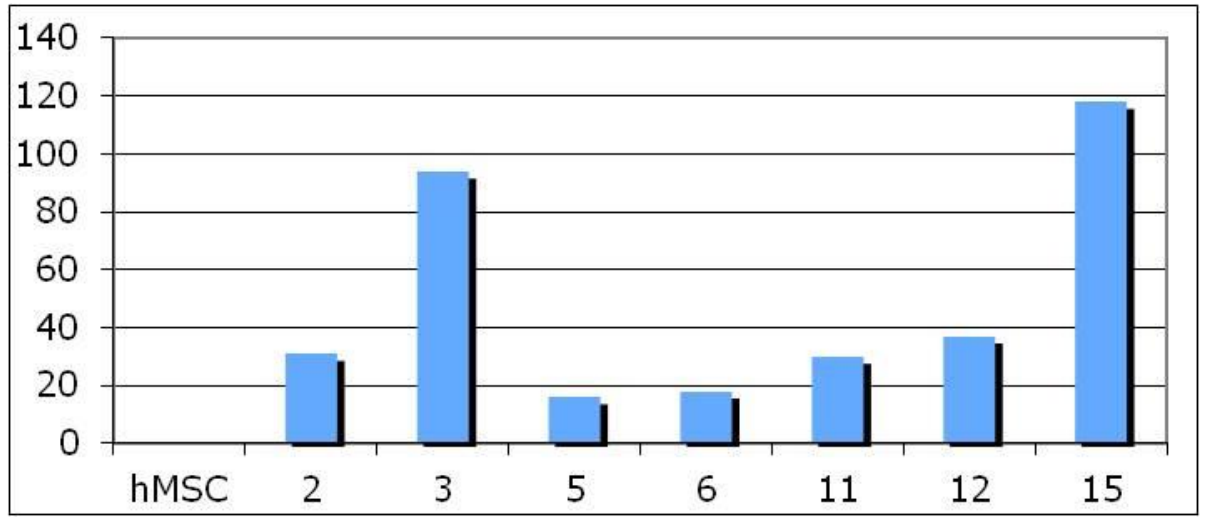

Figure 19. Quantification of CAG-CD55 expression levels of adMSC cell clones 2, 3, 5, 6, 11, 12 and 15. All values are relative to the CD55 expression of human immortalized MSC (SCP1) (=1). Expression was quantified using the primer combination CD55 qPCR F2/R2 and a CD55 specific TaqMan qPCR dual-labeled probe. As housekeeping gene GAPDH was measured using the primer combination poGAPDH F1/R1 and the probe poGAPDH. For the analysis of human CD55 expression levels the same primer/probe combination was used for CD55. For GAPDH, the same primer combination was used but with a probe specific for human GAPDH (hGAPDH).

\subsubsection{The CD46 minigene}

This section describes the various cloning steps necessary for the construction of the CD46 minigene containing several silent nucleotide substitutions. This CD46 minigene was chosen because animals transgenic for this construct have already been intensively characterized and served as the basis for CD46-hTM double transgenic animals, which have provided the most successful pig to baboon heterotopic heart xenotransplantation so far (Mohiuddin et al., 2014).

To increase translation efficiency of the CD46 minigene according to Milland et al. (1996), two A+T rich regions of the CD46 cDNA were modified by silent nucleotide substitutions in exons 3, 4 and 5. This increase was mainly due to reduction of the $\mathrm{A}+\mathrm{T}$ content rather than codon optimization. The $\mathrm{A}+\mathrm{T}$ content of the first region was reduced from $78 \%$ to $55 \%$ and one putative polyadenylation signal of the second region was disrupted. These modifications were performed using SOE-PCR reactions and showed to significantly increase levels of the membrane bound as well as soluble CD46 isoforms compared to the wild-type sequence (Milland et al., 1996). Table six shows the sequence of the different primers used for the SOE-PCR reactions. 
Table 6: Primer sequences for the SOE-PCR reactions. Mutated nucleotides are shown in red and restriction sites are shown in lower case (according to Milland et al., 1996).

\begin{tabular}{ll}
\hline Primer & Sequence \\
\hline On231 & 5'-CAATCAGGTAGTAACCCTCGTTGCAGATGAAGTGCATC-3' \\
\hline On232 & 5'-cggaattcACAGCGTCTTCCGCGCCGCGC-3' \\
\hline On233 & 5'-GGTCTTGTGTACACCACCTCCAAAGATCAAGAATGGAAAAC-3' \\
\hline On241neu & 5'-acgaattcGCAAACCTTTCTCTCATCTCTCC-3' \\
\hline On247 & 5'-GGTGGTGTACACAAGACCTTCTCACATATTGG-3' \\
\hline On250 & 5'-CGAGGGTTACTACCTGATTGGTGAGGAGATCCTGTATTGTGAAC-3' \\
\hline
\end{tabular}

As template for the SOE-PCR reactions, the cDNA of the CD46 $\mathrm{BC} 1$ isoform was amplified from the cDNA, prepared from the human MSC cell line SCP1 and cloned into the EcoRV restriction site of the pcDNA3.1-hygro(+) plasmid. For the first SOE-PCR, the primer combination On232/On247 was used to amplify the 5' PCR fragment and the primer combination On233/On241neu was used to amplify the 3' PCR fragment. For the substitutions in the SCR3 both PCR fragments were used together as templates with the primer combination On232/On241neu. The SOE-PCR fragment was cloned into the EcoRV restriction site of pcDNA3.1-hygro(+) and used as a template for the additional substitutions in SCR2. For these substitutions the 5' PCR fragment was amplified with the primer combination On232/On231. For the 3' PCR fragment the primer combination On250/On241neu was used. The SOE-PCR was performed using the primer combination On232/On241neu. The cDNA of CD46 with silent nucleotide substitutions in exons 3, 4 and 5 (SCR2+3) was cloned into the EcoRV restriction site of the pcDNA3.1-hygro(+) plasmid and could be subsequently to clone the CD46 minigene (Fig. 20). 


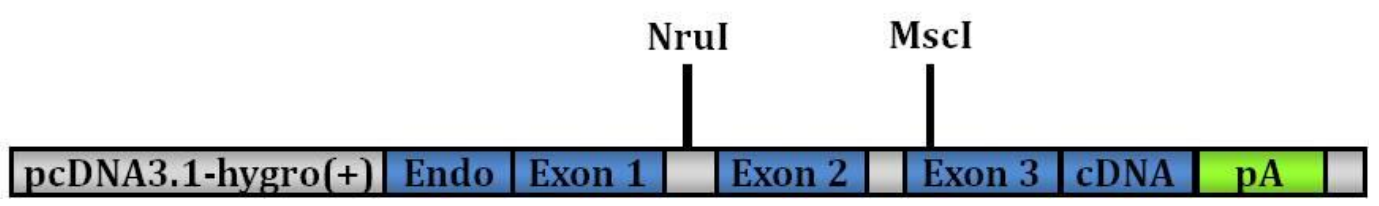

Figure 20. Structure of the CD46 minigene containing a 947 bp endogenous CD46 promoter, exon 1, intron 1, exon 2, intron 2 and parts of exon 3 of the genomic CD46 sequence and the cDNA sequence of CD46 ranging from exon 3 to exon 14. Silent nucleotide substitutions were engineered in exons 3,4 and 5 to increase protein expression.

For the construction of the CD46 minigene, a genomic region, stretching from the NruI restriction site of intron 1 to the NotI restriction site of exon 3, was amplified from the CD46-BAC construct using the primer combination CD46miniPCR2F/CD46miniPCR2R. This $3.2 \mathrm{~kb}$ PCR fragment was NruI and NotI digested and cloned into the NruI and NotI digested pcDNA3.1-hygro(+) plasmid. For the next step, this plasmid was XhoI and partial MscI digested. The $8 \mathrm{~kb}$ fragment was ligated with the XhoI and MscI digested PCR fragment using the primer combination CD46miniPCR3F/CD46miniPCR3R which contains the CD46 cDNA sequence (with silent nucleotide substitutions) from the MscI restriction site of exon 3 to exon 14. For the last cloning step of the CD46 minigene, a PCR containing 947 bp of the endogenous CD46 promoter, exon 1 and a part of intron 1 up to the NruI restriction site of intron 1 was amplified out of the CD46BAC construct using the primer combination CD46miniPCR1ePF/CD46miniPCR1R. This PCR fragment was NruI digested and cloned into the NruI restriction site of the pcDNA3.1 plasmid containing additional sequences of the CD46 minigene.

AdMSCs were transfected, cell clones picked and RNA isolated. Expression of the CD46 minigene was measured using Q-RT-PCR. Expression values were calculated and revealed high expression levels of the CD46 minigene (Fig. 21). The highest expressing clones reached more than 18-fold higher expression levels of CD46 compared to human MSCs. 


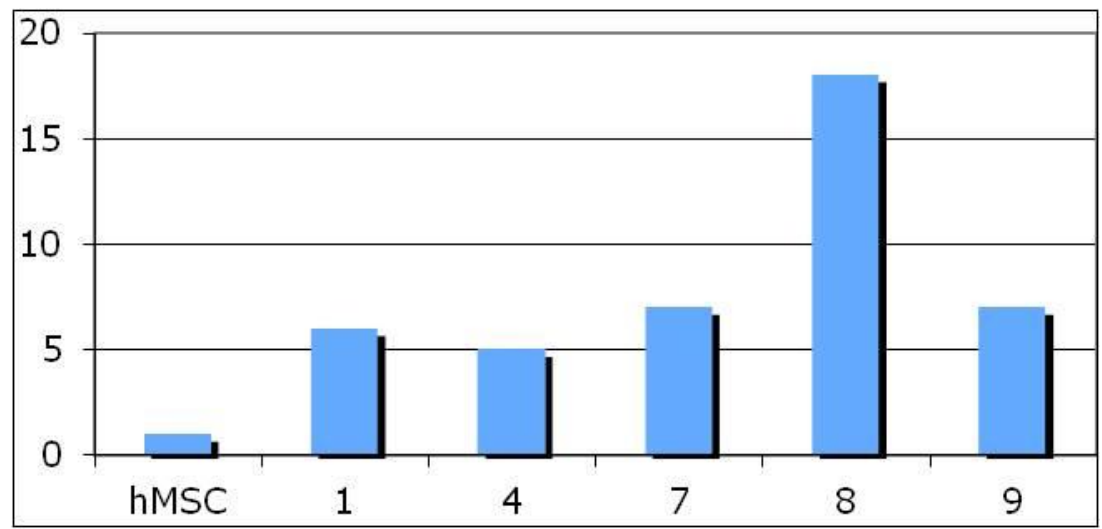

Figure 21. Quantification of CD46 expression levels of adMSC single cell clones 1, 4, 7, 8 and 9. All values are relative to the CD46 expression of human immortalized MSC (SCP1) (=1). Expression of CD46 was measured using the primer combination CD46 2nF/3cR and the CD46 specific TaqMan dual-labeled probe. GAPDH was used as housekeeping gene and detected using the primer combination poGAPDH F1/R1 and a porcine GAPDH specific probe. Expression of human MSC (SCP1) was measured using the same primer/probe combination for CD46. The primer combination poGAPDH F1/R1 and the probe for human GAPDH were used for detection of GAPDH.

\subsubsection{The CD39 cDNA construct}

Besides regulation of the complement system, control of the recipient's coagulation system is also necessary to inhibit xenograft rejection. Besides human thrombomodulin also human CD39 can offer means of coagulation control. CD39 hydrolyzes ATP and ADP to AMP, which is then converted to adenosine by CD73. This causes a shift from an ATP-driven proinflammatory state to an anti-inflammatory state. Moreover, the ADP-induced platelet aggregation and thrombus formation is abolished.

Therefore, a CD39 cDNA construct was generated. Although cDNA constructs are more likely to undergo gene silencing than minigenes, it was a convenient first option as a full length human CD39 cDNA (accession number BM 925184) was commercially available (Source BioScience).

This plasmid was RsrII and XhoI digested and the $1.7 \mathrm{~kb}$ CD39 cDNA cloned into the NruI and NotI digested pcDNA3.1-hygro(+)-CAG plasmid. AdMSCs (Isolation number 101212 and 1604) were transfected and selected using Hygromycin. Single cell clones were picked and expanded. 
Expression of CD39 was verified using RT-PCR and Q-RT-PCR analysis. Q-RT-PCR analysis revealed up to 70 fold higher expression of human CD39 RNA in porcine cells compared to human MSC (Fig. 22).

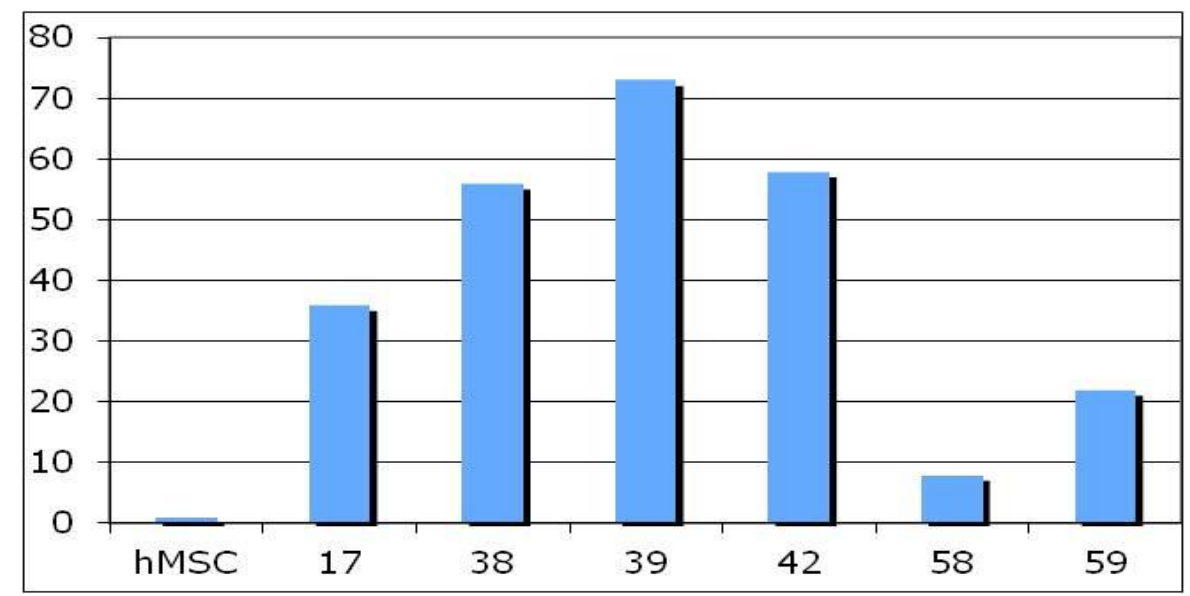

Figure 22. Quantification of CD39 expression of adMSC cell clones 17, 38, 39, 42, 58 and 59. Values are expressed relative to CD39 expression of human immortalized MSC line SCP1 (=1). CD39 was detected using the primer combination CD39 Ex5F/Ex6R and a CD39 specific TaqMan dual-labeled probe. GAPDH was used as housekeeping gene and detected using the primer combination poGAPDH F1/R1 and a porcine GAPDH specific probe. Expression of human MSC (SCP1) was measured using the same primer/probe combination for CD39. The primer combination poGAPDH F1/R1 and the probe for human GAPDH were used for detection of GAPDH.

\subsubsection{HBXIP}

In the previous sections different modified BAC and PAC constructs as well as minigenes of the complement regulatory genes CD46, CD55 and CD59 were described. These constructs were optimized to achieve high expression. The following section describes an attempt to additionally increase expression levels of all complement regulatory genes.

Cui et al. reported 2012 that the hepatitis B X-interacting protein (HBXIP) is able to upregulate expression of the complement regulatory proteins CD46, CD55 and CD59 in breast cancer cells via p-ERK1/2NF- $\kappa B$ signaling. Moreover, it has been reported that HBXIP in combination with the tumor-specific protein SURVIVIN is able to suppress apoptosis (Marusawa et al., 2003). 
The HBXIP-SURVIVIN complexes selectively suppress apoptosis via the michochondria/cytochrome c pathway by binding pro-caspase 9. I therefore investigated whether cotransfection of this gene could increase expression of human complement regulatory genes and whether there were any anti-apoptotic effects in adMSCs. As the function of the human HBXIP in porcine cells has not been investigated so far, it was first necessary to establish whether HBXIP is functional in porcine cells. If HBXIP is able to upregulate expression of human complement regulatory genes, it also should be able to upregulate porcine complement regulatory genes via the same pathways. The pCMV-Sport6 vector was ordered containing the human HBXIP cDNA cloned into the NotI and EcoRV restriction site (Source Bioscience, ID 5190406/AT82 E08 M13R). As this plasmid lacked a selectable eukaryotic marker, a BS-Neo cassette was added. For this purpose, the plasmid PL452inf-BSinf was NotI and KpnI digested and the BSNeo cassette was cloned into the pCMV-Sport6-HBXIP vector (Nsil digested). The resulting plasmid was transfected into adMSCs. Cells were selected using BS for 7 days. Cell clones with detectable HBXIP expression (Fig. 23) were chosen. Expression levels of the endogenous porcine CD55 gene in these cell clones and the corresponding control (wild type adMSCs) were determined (Fig. 24). As shown, the expression of HBXIP in porcine cells did not influence expression of porcine CD55. Moreover, high HBXIP expression did not correlate with increased porcine CD55. Thus, the results of Cui et al. 2012, obtained using human breast cancer cells, could not be confirmed in porcine cells. 


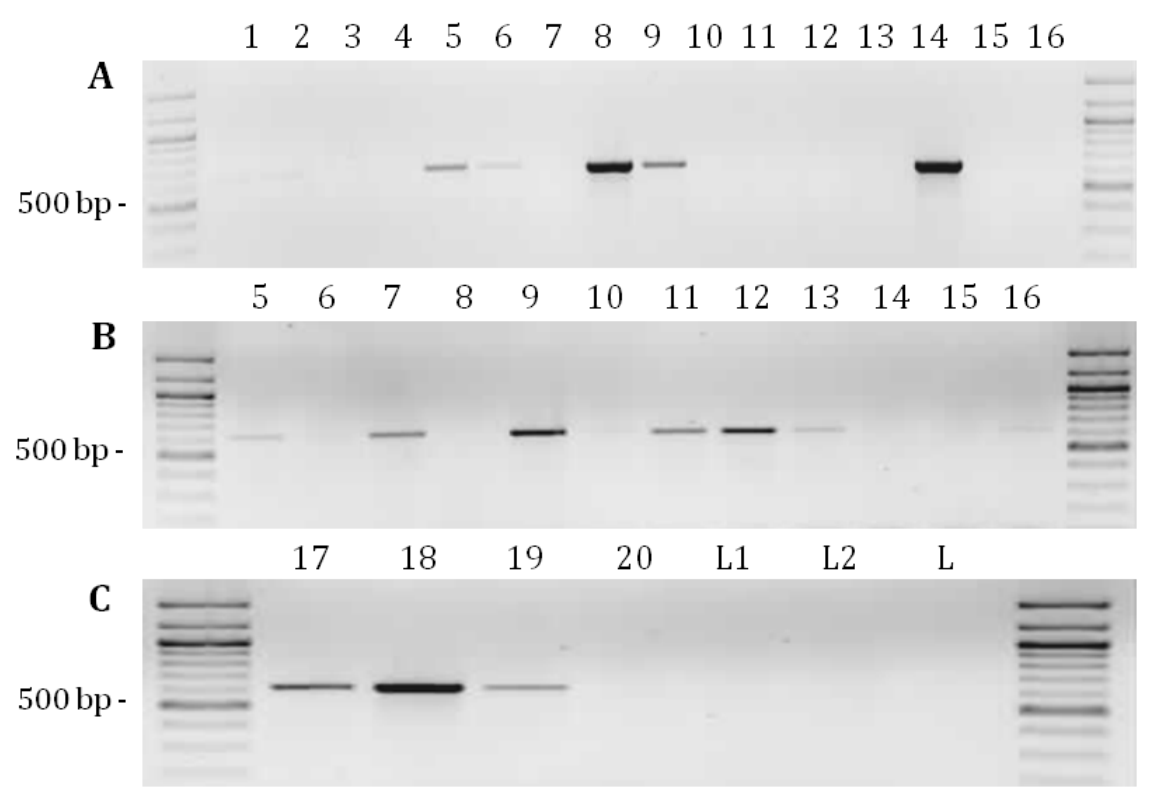

Figure 23. RT-PCR analysis of HBXIP expression from different adMSCs (A: Isolation number 3011; B,C: Isolation number 0102). The primer combination HBXIP F1/R1 (630 bp) was used. L1: untransfected adMSC (Isolation number 3011). L2: untransfected adMSC (Isolation number 0102). L: water.

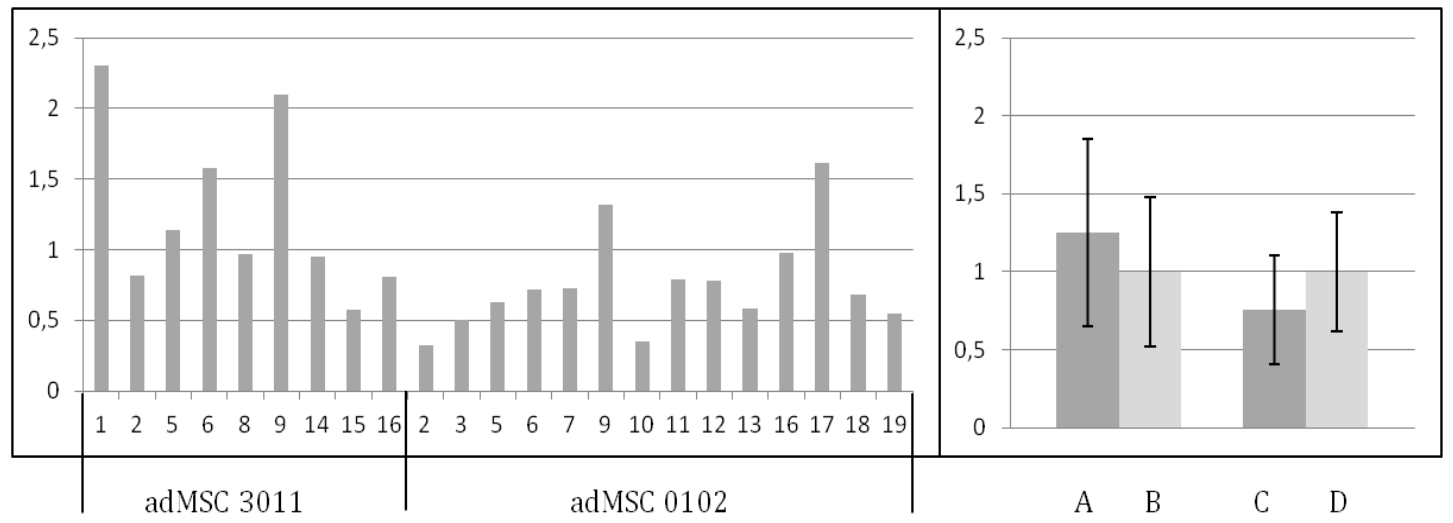

Figure 24. Left: Quantification of porcine CD55 expression of HBXIP transfected adMSC (Isolation number 3011 and 0102) referred to the average porcine CD55 expression (=1) of wild type adMSC (Isolation number 3011 or 0102). Right: Average porcine CD55 expression and standard deviation. A: HBXIP transfected adMSC $3011(n=9)$. B: Wild type adMSC $3011(n=12)$. C: HBXIP transfected adMSC 0102 (n=14). D: Wild type adMSC 0102 (n=12). 


\subsection{Xeno-HAC formation and transfer}

Human artificial chromosome (HAC) vectors can be used as a means of delivering xenoprotective transgenes. HACs can carry whole sets of transgenes independent of the host genome, thus avoiding any effects due to the chromatin environment. The formation of HACs has however only been successful in cells with high telomerase activity such as human HT1080 cells, and has so far never been successful in porcine cells. It is thus necessary to transfer the HAC from the human line where it was generated into porcine cells. Microcell-mediated chromosome transfer has previously been used among other things for the generation of transchromosomal animals via the transfer of a human artificial chromosome from human into murine or bovine cells (Tomizuka et al., 1997; Suzuki et al., 2006). I investigated whether this technique could be established using porcine cells.

The first obstacle was the relatively short life span of porcine primary somatic cells, too short to carry out fusion and perform complete analysis (including FISH analysis) of clones after microcell-mediated chromosome transfer.

\subsubsection{Immortalization of cells}

To extend the life span of cells several methods are possible. Proto-oncogenes can be activated, tumor suppressor genes can be inactivated and several viruses are available for the immortalization of cells. Here, the expression of human telomerase reverse transcriptase (hTERT) was used to increase the lifespan of cells. Moreover, Neo expressing cells were beneficial to enable Neo-BS double selection after microcell-mediated chromosome transfer as the HAC vector carried a BS resistance gene. AdMSCs 1101 and bone marrow MSC 1001 were nucleofected with the plasmid pCl-Neo-hTERT. A control group was transfected with a SV40-neomycin resistance cassette which is also part of the pCl-NeohTERT plasmid. All cells were selected with neomycin until cell clones were visible. Selection was stopped and clones were further expanded.

RT-PCR was used to analyze the hTERT expression from pools of the hTERT transfected cells and the control group after 14 days (Fig. 25). 


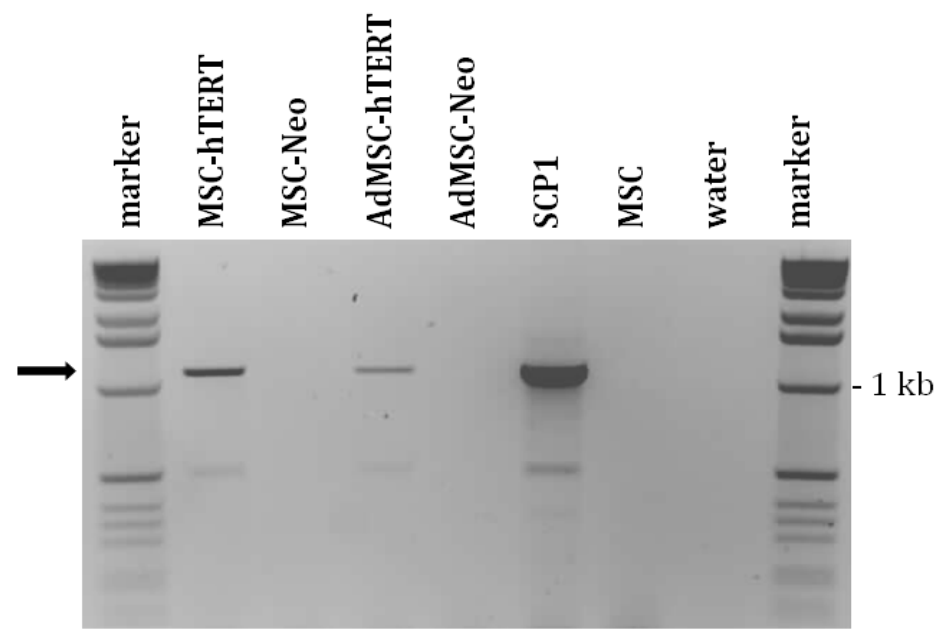

Figure 25. RT-PCR expression analysis of hTERT transfected cells and a control group. HTERT expression was confirmed by a $1.1 \mathrm{~kb}$ band. The primer combination hTERTcDNA F1/R1 was used. MSC-hTERT: hTERT transfected MSCs. MSC-Neo: Neomycin transfected MSCs as negative control. AdMSC-hTERT: hTERT transfected adMSCs. AdMSC-Neo: Neomycin transfected adMSCs as negative control. SCP1: hTERT expressing cell line SCP1 as positive control. MSC: Untransfected MSCs as negative control.

RT PCR results confirmed hTERT expression in the stable transfected bone marrow MSC and adMSC pools. To examine the effects of hTERT expression, a colony forming assay was conducted after 14, 28 and 56 days. 480 cells of the hTERT expressing cells or the control group were plated per $10 \mathrm{~cm}$ dish. The number of cell clones was determined 7 days after plating.

After 14 days, the hTERT transfected cells showed 12\% (MSC) or 21\% (adMSC) more colonies compared to the control group. As colony size could not be determined directly, cells were detached from the plate and the total number of cells was determined. MSC showed a 15\% and adMSC a 27\% higher number of cells compared to the control group. The colony forming assay was repeated after 28 and 56 days to confirm an extended lifespan of the cells. However, the MSCs and adMSCs showed almost no difference to the control group. The number of colonies after 28 days was almost equal $(+3 \%$ for MSC and $+2 \%$ for adMSC) and the cell number was just slightly higher (+1\% for MSC and $+3 \%$ for $\operatorname{adMSC})$.

After 56 days, the number of colonies was 2\% lower for MSC and 1\% higher for adMSC compared to the control group. The cell number was 7\% lower for MSC and $1 \%$ higher for adMSC. 
Transfection of MSC and adMSC using hTERT thus showed only a short term effect as measured by this colony forming assay. Successful immortalization of the cells could not be detected.

\subsubsection{HAC transfer using MMCT}

This section describes the method of microcell mediated chromosome transfer (MMCT) which is necessary to transfer a HAC which was assembled in human HT1080 cells into porcine cells. This method can also be used to transfer altered human chromosomes carrying several xenograft-protective genes into porcine cells. As HAC formation in porcine cells has not yet been successful, the HAC has to be first formed in cells such as human HT1080 cells and subsequently transferred. The methods to form microcells were developed by Ege and Ringertz, 1974. Fournier and Ruddle (1977) were the first to use these methods to form viable mouse microcell hybrids.

MMCT has been used in many fields to assess genomic stability, imprinting and other chromatin modifications as well as genomic structure and genome organization (Meaburn et al., 2005). The method of MMCT has been very successful for intra-species chromosome transfer, but less so for inter-species chromosome transfer. The transfer efficiency and the stability of the HAC in the recipient cell line depends very strongly on the centromere sequence and the genetic background (Vissel, Choo 1991).

To optimize MMCT, the number of HPRT-HACs in the donor HT1080 cell line (EHG6k) which is a subclone of the original EHG6 HT1080 cell line, harboring two HACs with the human HPRT gene (Bieringer, 2008) had to be determined. Chromosome spreads were prepared and FISH was performed using probes detecting the HAC specific centromeric structures (E1deac) and the HPRT gene (HPRT-bio). In total 95 different metaphase spreads were analyzed. In $72 \%$ of all photomicrographs two HACs per cell were visible and in $28 \%$ one HAC was visible (Fig. 26). 

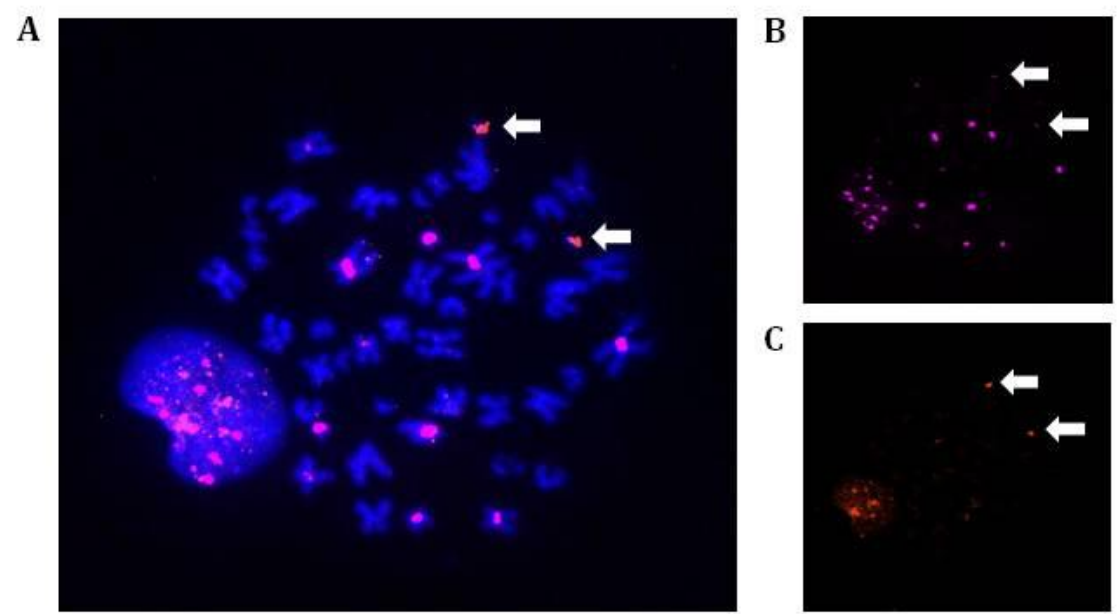

Figure 26. FISH analysis of the HT1080 cell line EHG6k. A: Metaphase chromosome spread hybridized with fluorescent probes E1deac (pink; specific for centromeric sequences) and HPRTbio (red; specific for the HPRT gene) revealed presence of two HACs. B: Fluorescent probe E1deac (pink). C: Fluorescent probe HPRT-bio (red). The two human artificial chromosomes are marked with arrows.

Subsequently the MMCT was performed using human HT1080 cells (EHG6k) as donor cell line and either porcine ST007 cells or porcine MSCs, both carrying a neomycin resistance gene, as recipient cell line. Recipient cells carrying a neomycin resistance were chosen as previous experiments to increase the lifespan of porcine somatic cells were unsuccessful (see previous section). As the HAC contained an EGFP cassette, fluorescence of cells was checked two days after performing the MMCT, giving a first hint of a successful HAC transfer (Fig. 27). 
A

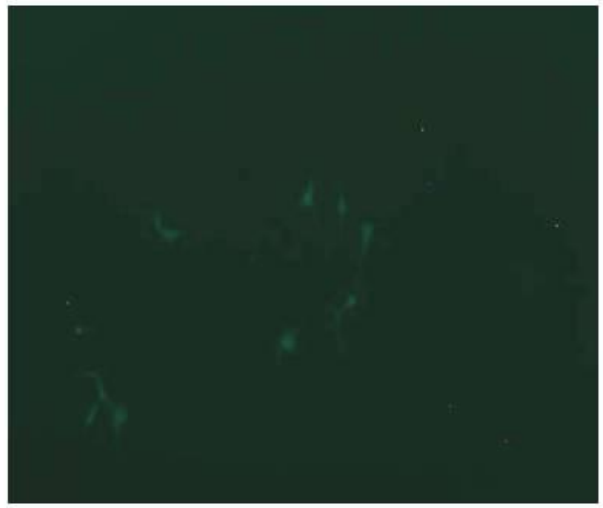

C

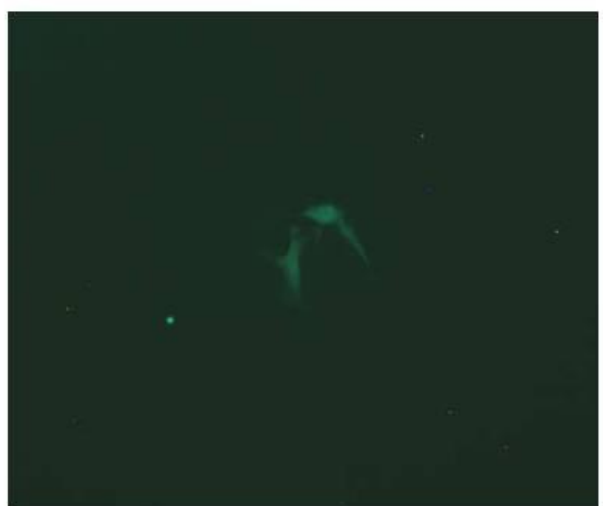

B

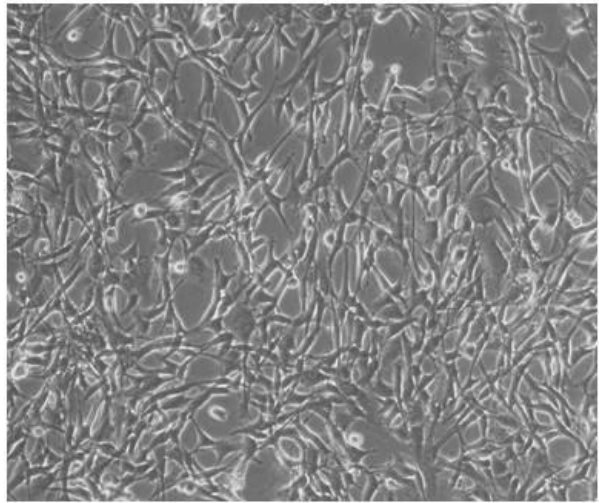

D

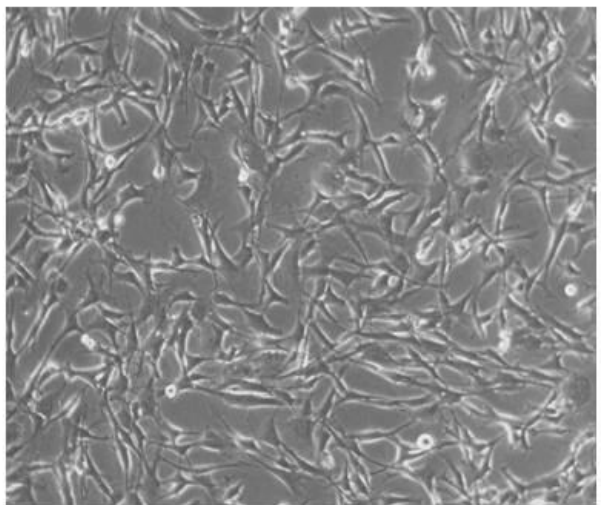

Figure 27. Transient EGFP expression of porcine MSC two days after MMCT. A and C: EGFP fluorescence of pMSCs. B and D: Corresponding pMSCs under transmitted light.

Subsequently, the recipient cells were selected using a G418-BS double selection as a BS resistance cassette was located on the HAC. The selection of the recipient cells using only BS was not possible as in all experiments conducted, a very small amount of viable HT1080 cells was transferred to the recipient cells. As HT1080 cells grow much faster than the porcine cells, a double selection was essential to receive a pure population of porcine cells containing a transferred HAC.

After selection, clones were picked and analyzed via PCR using the primer combinations AmF/BemF, PBSF/PEGFPR and PBS2F/PBS2R detecting the HAC centromeric region, EGFP or the BS cassette. Some ST007 and MSC clones showed a successful HAC transfer. To exclude an additional human chromosome transfer, PCRs were conducted, screening for human CD46, CD55, CD59, HO, A20 and $T M$ which are located on chromosomes 1,1,11,16/22, 6 and 20. The primer combinations CD46 2cF/3cR were used for the detection of CD46, CD55 7cF/8cR for CD55, CD59 3cF/3cR for CD59, HMOX 3cF/3cR for HO, hA20 7cF/8cR for A20 and $\mathrm{TMncF} / \mathrm{ncR}$ for the detection of TM. 
All MSC clones possessed many additional human chromosomes. Some of the ST007 clones revealed no additional human chromosomes. The ST007 clones and some MSC clones were further analyzed by FISH to verify the PCR results. FISH images were taken more than 90 days after MMCT to ensure a stable HAC transfer. Figures 28 and 29 show several metaphase spreads. The MSC clone M9 had 4 transferred HACs (visible in 5/5 pictures) but also several additional human chromosomes. The examined ST007 clones had no additional human chromosomes. ST007 clone S6 had one transferred HAC (visible in 19/21 pictures) and the ST007 clone S14 had 2 transferred HACs (visible in 17/17 pictures).

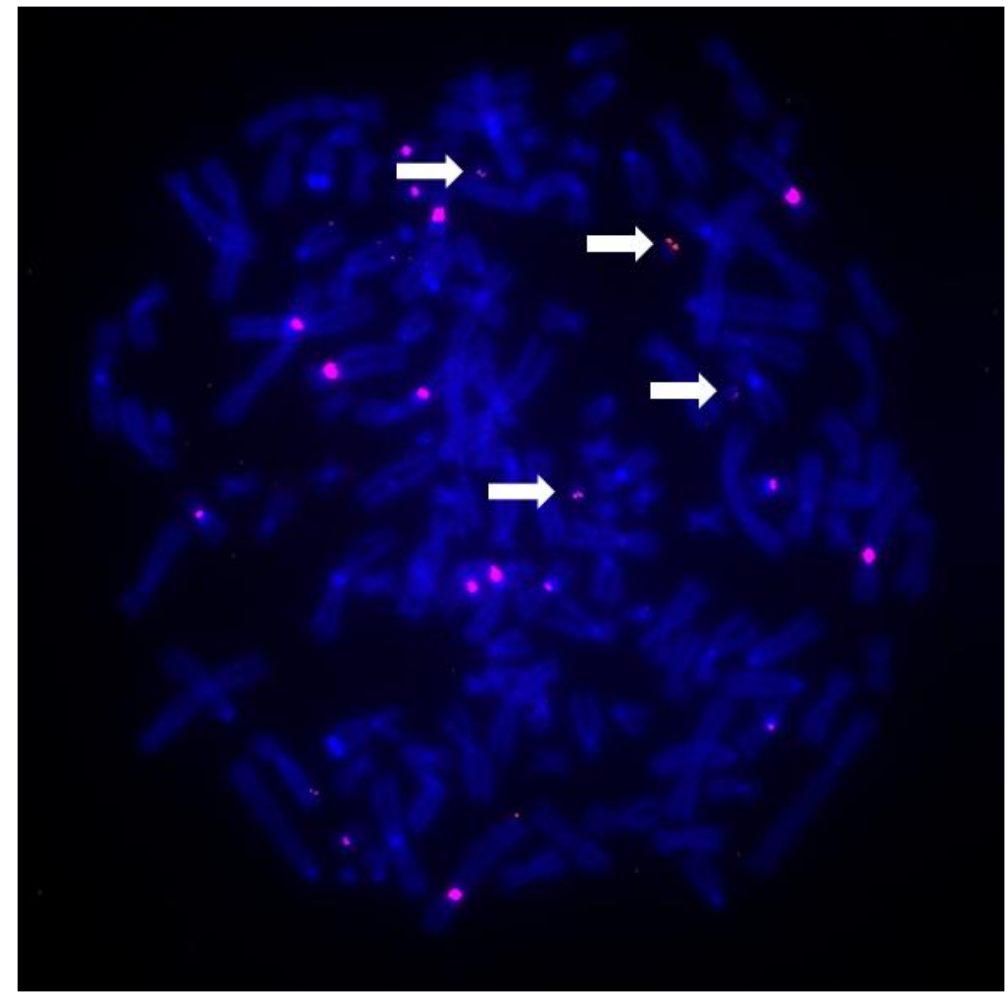

Figure 28. Metaphase chromosome spread of MSC clone M9 hybridized with fluorescent probes E1deac (pink) and HPRT-bio (red). Four transferred human artificial chromosomes are marked with arrows. 

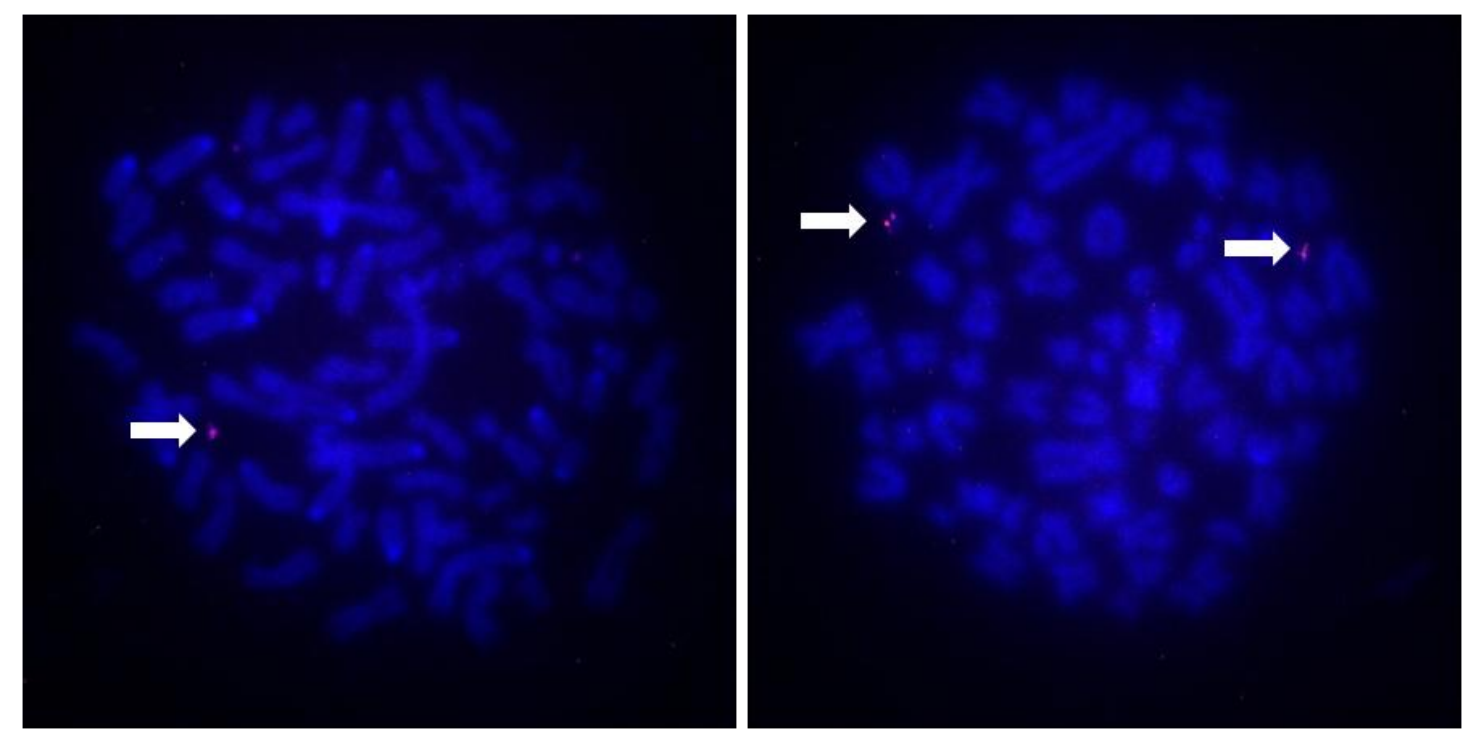

Figure 29. Metaphase chromosome spreads of ST007 clones S6 (left) and S14 (right) hybridized with fluorescent probes E1deac (pink) and HPRT-bio (red) confirming one or two transferred human artificial chromosomes. HACs are marked with an arrow.

This experiment revealed that the method of MMCT had successfully been optimized to enable an interspecies chromosome transfer between human and porcine cells. Although the formation of a HAC remains very challenging, the method of MMCT could additionally be used to transfer altered human chromosomes, carrying whole sets of graft-protective xenogenes, into porcine cells.

\subsubsection{Formation of xeno-HAC}

The following section focuses on attempts to create a human artificial chromosome (HAC) in human HT1080 cells. This HAC should carry several complement regulatory and additional xenograft protective transgenes. An important characteristic of human artificial chromosomes is their stable episomal maintenance. Thus, they do not integrate into the host genome and cause disruption of genes or random integration and random expression of transgenes. Moreover, HACs have the capacity to carry huge genomic constructs with all their regulatory elements and enable physiological regulation of gene expression (Oshimura, Katoh 2008). 
Xeno-HACs containing the genomic sequences of several complement regulatory genes and other important xeno-genes could be very helpful for the production of multi-transgenic animals and ensure the reliable expression of xeno-genes. For this reason, an approach was used to achieve a de novo xeno-HAC formation. Previous experiments showed that the formation of a HAC is possible in human HT1080 cells, which express telomerase (Barnett et al., 1993; Hanish et al., 1994; Laner, 2005). Previous experiments at the chair to achieve HAC formation in porcine cells had failed. All cotransfections of various combinations of linearized xeno-constructs (CD55-XS, CD55-TM-Lea, CD55-HO-A20, GAG-CD55-XS, CAGCD55-XS-TM-Lea, CAG-CD55-S-H0-A20, CD46-M, CD59-M) were performed in HT1080 cells together with 10-200 ng NotI-digested, tetra-telomerised PAC construct (pTTE1) containing $116 \mathrm{~kb}$ of human chromosome 5 alpha satellite DNA, a CMV/EGFP expression cassette and two BS resistance genes (Laner et al., 2004). The pTTE1 plasmid was digested in the plug, separated via PFGE, excised and subsequently electroeluted. Figure 30 shows the structure of the HAC vector pTTE1.

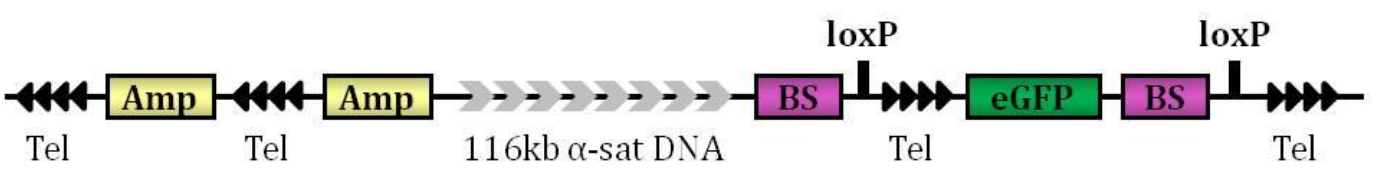

Figure 30. Tetra-telomeric (Tel) pTTE1 vector with ampicillin resistance (Amp), BS resistance, two loxP-sites and enhanced green fluorescing protein (eGFP).

Earlier experiments at the Chair of Livestock Biotechnology for the production of HAC boars revealed that human alpha satellite DNA might not be stable in transgenic pigs. As a result, cotransfections of the pTTE1 construct, xeno-genes and porcine centromeric sequences were performed in HT1080 cells to ensure the functionality of the centromeric region in porcine cells. Therefore, 30 different samples containing porcine centromeric sequences were tested for the Mc1 specific porcine centromeric subunit of 340 bp (Janzen et al., 1998) using the primer combination Pocem1F/R. The constructs SCIV51 and SCIV115 (Schäffauer, 2008) which contain the pTT vector and porcine centromeric sequences were further used. 
A BamHI digest was conducted to delete the vector backbones. The DNA was subsequently separated via PFGE and electroeluted. As SCIV 51 delivered a better DNA quality, the $\sim 55 \mathrm{~kb}$ centromeric sequence of this plasmid was used for the cotransfection experiments.

After the cotransfection and selection, cell clones were picked and several PCRs were conducted to detect HAC specific regions and to preclude deletions using the primer combinations AmF/BemF, PBSF/PEGFPR and PBS2F/PBS2R. Subsequently FISH analysis was performed to examine whether a HAC formation of the pTTE1 construct together with the cotransfected xeno-genes occurred. However, just random integration events of the pTTE1 vector and the xenogenes without any successful HAC formation could be detected. Therefore this strategy was abandoned.

\subsection{Multi transgenic cells and animals}

As an alternative strategy to episomal HACs carrying multiple xeno-genes, multitransgenic cells were to be generated, selected for high expression and if successful, cloned animals were to be produced.

\subsubsection{Generation of multi-transgenic porcine cells}

The multi-gene BAC constructs of CD55 (CAG-CD55-XS-HO-A20 and CD55-XSTM-LEA), driven by the CAG or the endogenous CD55 promoter, were subsequently used in combination with the CD46-L BAC construct and the CD59M PAC constructs, both driven by their endogenous promoter sequences, for the transfection of porcine cells. The general aim was to produce multi-transgenic porcine cells. Bone marrow MSC, adMSC and GGTA1 knockout fibroblasts were cotransfected with various xeno-gene constructs.

After transfection and selection, several hundred cell clones were picked, expanded and expression was analyzed using RT-PCR. Multi-transgenic cell clones were identified which showed different combinations of xeno-gene expression (Fig. 31-33). 
One GGTA1 knockout fibroblast clone (C1F6-2) showed expression of six different xeno-genes (CD46, CD55, CD59, LEA29Y, A20 and H01). Only few adMSC clones revealed high expression levels especially of the complement regulatory genes (clone 3-6 and 4-39; Transfection conducted by S. KranerScheiber) as well as other xeno-genes. As the major aim of this thesis was to identify cell clones with at least five fold higher expression levels of CD55 compared to human MSCs, clones 3-6 and 4-39 were used for further characterization and for somatic cell nuclear transfer to obtain transgenic animals. These cotransfection experiments confirmed that multi-transgenic clones can be obtained by cotransfections of BAC and PAC constructs.

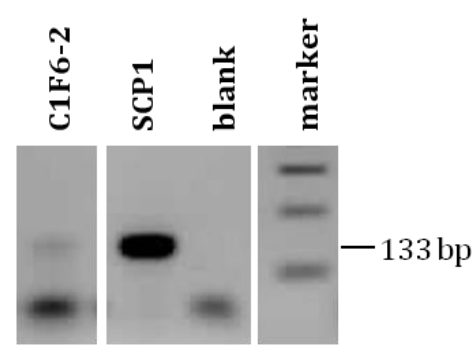

CD46

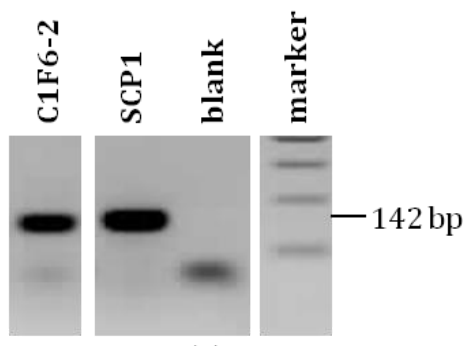

CD55

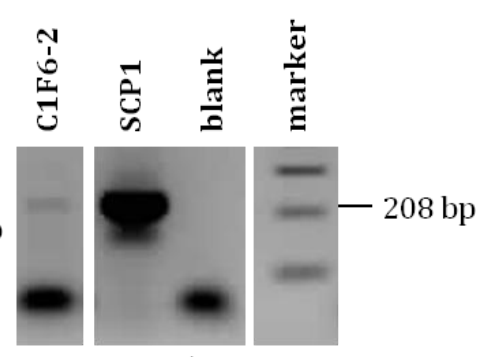

CD59

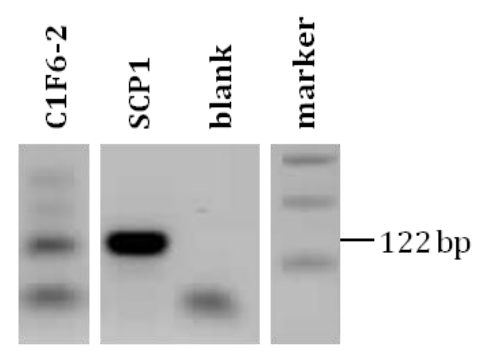

HO

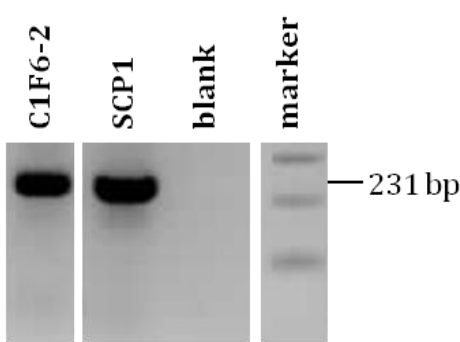

A20

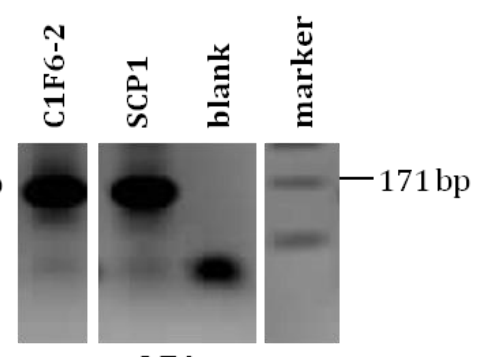

LEA

Figure 31. RT-PCR analysis of GGTA1 knockout clone C1F6-2 revealing CD46 expression (Primer combination CD46 2nF/3cR); CD55 expression (Primer combination CD55 qPCR F2/R2); CD59 expression (Primer combination CD59 3cF/5cR); HO1 expression (Primer combination HMOX 3cF/3cR); A20 expression (Primer combination (hA20 7cF/8cR) and LEA29Y expression (Primer combination LeaF7LeaExpr2R). SCP1 as positive control. 


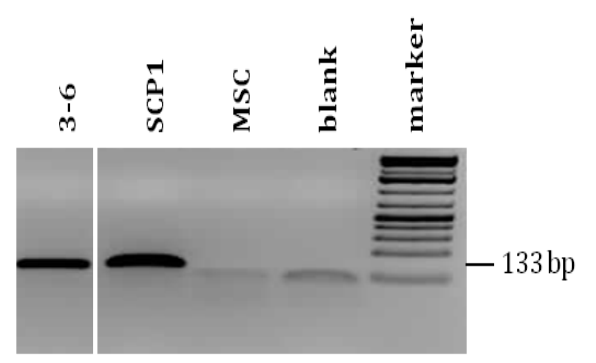

CD46

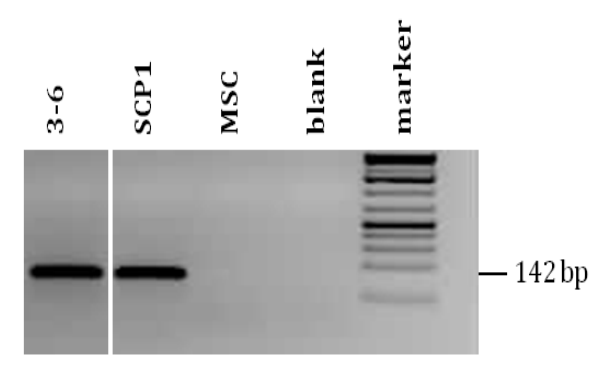

CD55

Figure 32. RT-PCR analysis of clone 3-6 revealing CD46 expression (Primer combination CD46 2nF/3cR) and CD55 expression (Primer combination CD55 qPCR F2/R2). SCP1 as positive control. MSC as negative control.

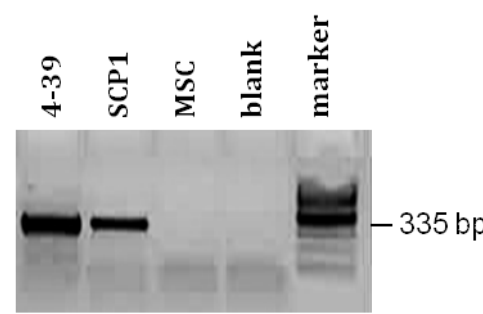

CD46

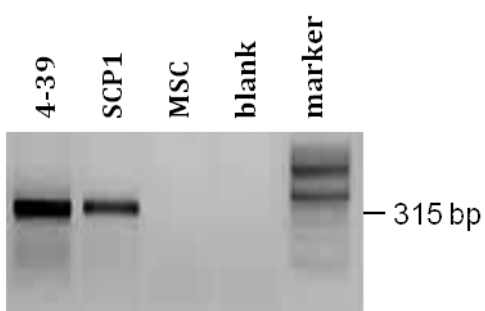

CD55

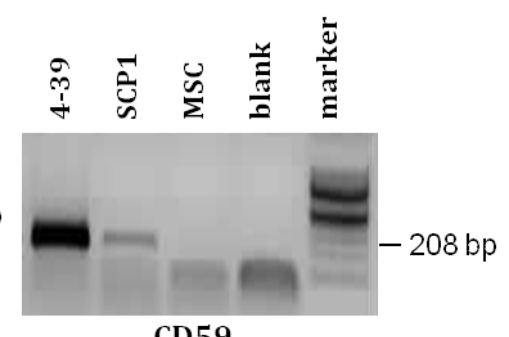

CD59

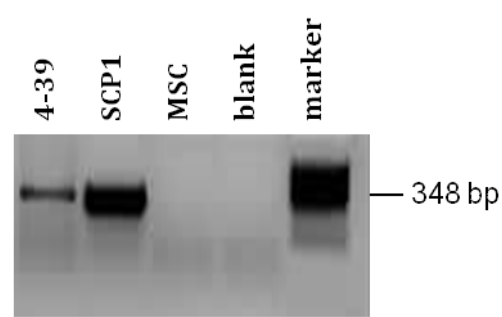

HO

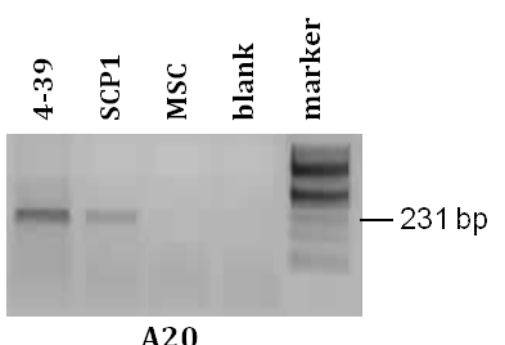

A20

Figure 33. RT-PCR analysis of clone 4-39 revealing CD46 expression (Primer combination hCD46 Ex3F1/Ex5R1); CD55 expression (Primer combination CD55 huF1/R2); CD59 expression (Primer combination CD59 3cF/5cR); HO1 expression (Primer combination hHOEx1F1-HO3cR) and A20 expression (Primer combination (hA20 7cF/8cR). SCP1 as positive control. MSC as negative control.

\subsubsection{Production and analysis of multi-transgenic pigs}

To obtain transgenic animals with a high expression level of the complement regulatory genes, cell clones 3-6 and 4-39 were used for somatic cell nuclear transfer (SCNT) which was conducted in collaboration with the group of Prof. Wolf, LMU. 
For clone 3-6, 253 reconstructed embryos were transferred to two recipient sows, one pregnancy established and one stillborn piglet (1107-6) obtained. For clone 4-39, 238 reconstructed embryos were transferred to two recipient sows, one pregnancy established and one piglet obtained (1706). Piglet 1706 was healthy and normally developed. However, this piglet died shortly after birth due to injury. Porcine kidney fibroblasts of both animals were cultured for functional analysis, additional genetic manipulations and for further rounds of SCNT to obtain living animals. For re-cloning of piglet 1107-6, 348 reconstructed embryos were transferred to four recipient sows, two pregnancies established and 11 healthy liveborn piglets and one stillborn piglet obtained. For re-cloning of piglet 1706, 552 reconstructed embryos were transferred to five recipient sows, one pregnancy established and one healthy liveborn piglet (266) obtained. Tissue samples from animals 1107-6 and 1706 were collected, RNA isolated and expression of the complement regulatory genes verified using RT-PCR. Figure 34 and figure 35 show the RT-PCR results of pig 1107-6 (M. Thiessen) and 1706 (B. Rieblinger). Both animals revealed high expression levels of the complement regulatory genes in all tissues examined. Subsequently, expression levels of the complement regulatory genes in animal 1706 were quantified using Q-RT-PCR analysis (Fig. 36). The CAG-driven CD55 construct revealed the highest expression levels in all tissues compared to the CD59 and CD46 constructs which were both driven by their endogenous promoter sequences.

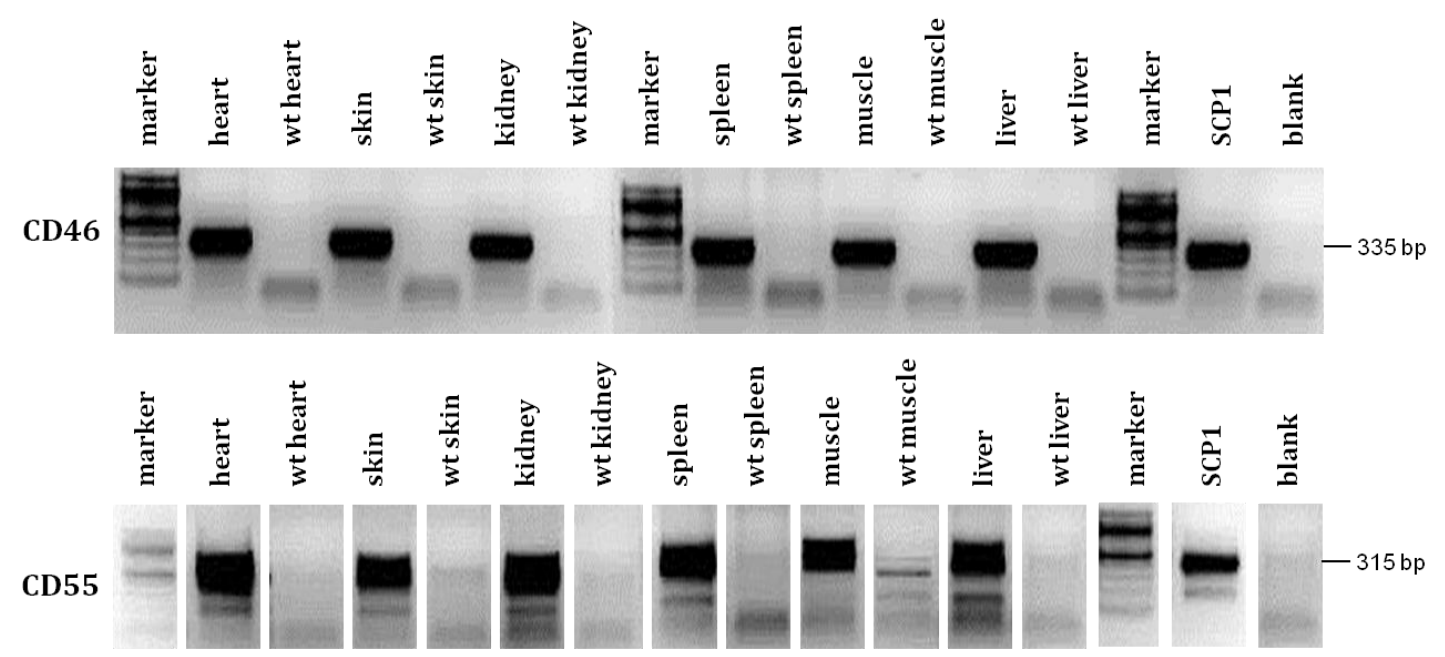

Figure 34. RT-PCR analysis of CD46 and CD55 expression in tissues of animal 1107-6. Primer combinations used: CD46Ex3F1/Ex5R1; CD55huF1/huR3. SCP1 as positive control. 


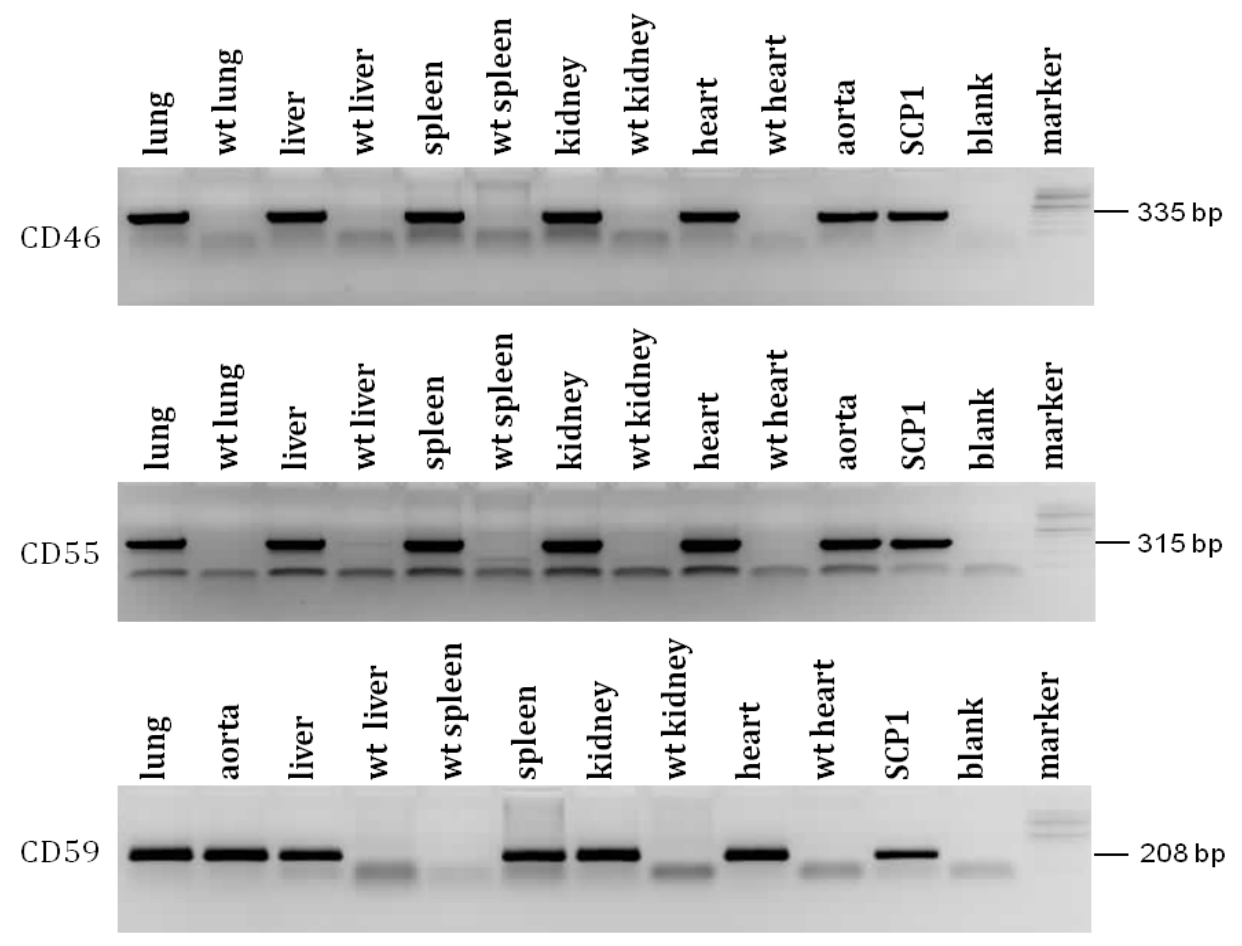

Figure 35. RT-PCR analysis of CD46, CD55 and CD59 expression in tissues of animal 1706. Primer combinations used: CD46Ex3F1/Ex5R1; CD55huF1/huR3; CD59 3cF/5cR. SCP1 as positive control.

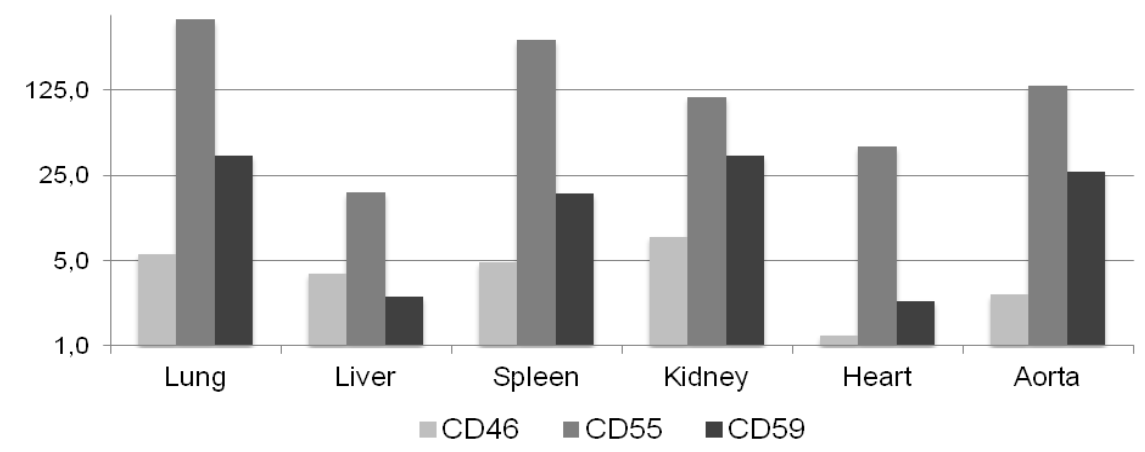

Figure 36. Q-RT-PCR analysis and quantification of CD46, CD55 and CD59 expression levels in tissues of animal 1706. Expression levels are shown relative to human MSC (SCP1; expression = 1).

As animal 1706 revealed high expression levels of all complement regulatory genes, metaphase chromosome spreads of kidney fibroblasts were prepared to identify the number of chromosomal integration sites. Fortunately, only one chromosomal integration site could be detected (Fig. 37). The exact locus of this integration site was further determined by Q-banding (I. Szczerbal and M. Switonski; University Poznan), mapping the integration site to chromosome 6q22 (Fig. 38). 
The number and exact determination of the integration site is very important for breeding of the animal to prevent segregation of the transgenes and to enable a retargeting approach to stack further xenogenes at this site.

A
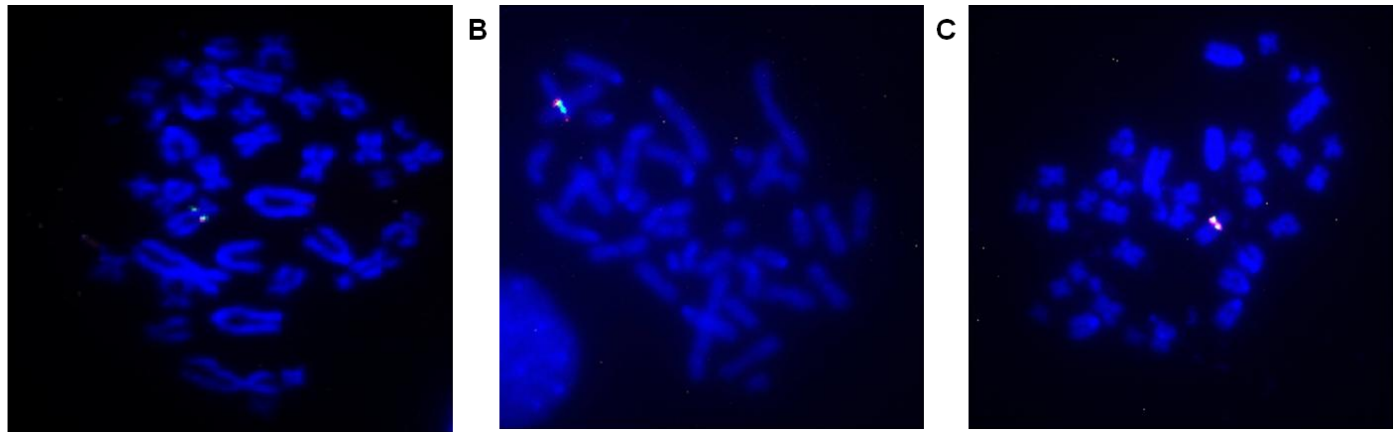

Figure 37. Metaphase chromosome spreads of kidney fibroblasts of animal 1706 revealing the integration site for A, CD46 and CD59. B, CD46 and CD55. C, CD46, CD55 and CD59. All cotransfected constructs integrated at a single locus.
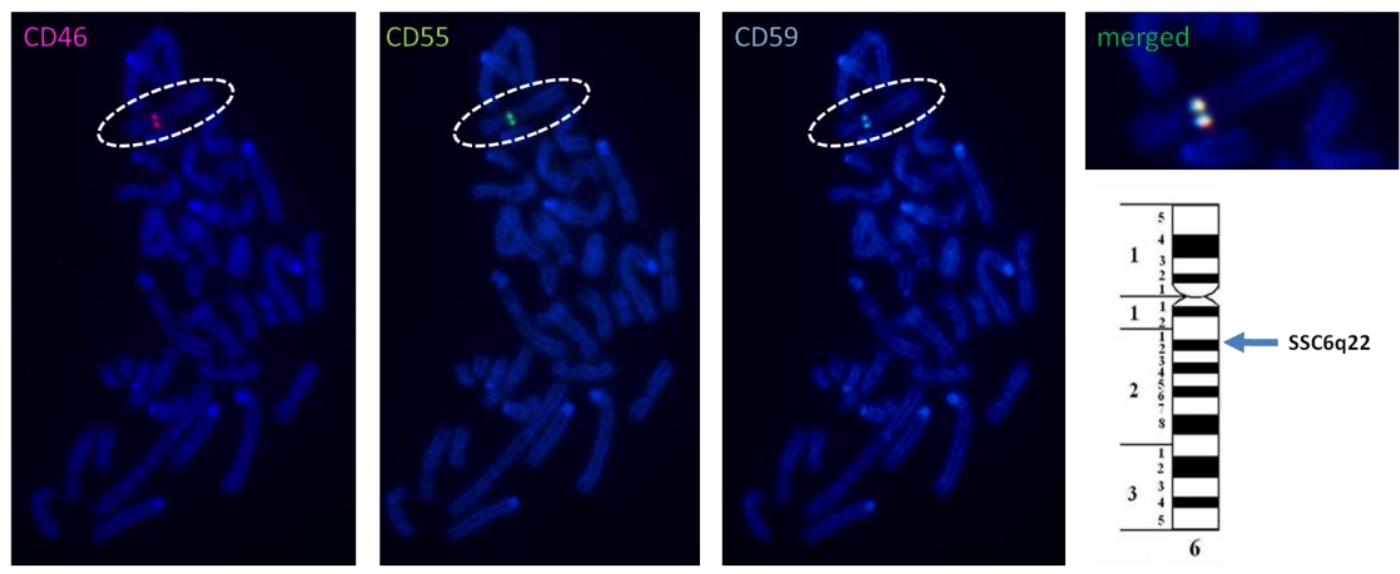

Figure 38. Metaphase chromosome spreads and Q-banding of porcine kidney fibroblasts from animal 1706 mapping the integration site of all cotransfected constructs to chromosome 6q22.

Porcine kidney fibroblasts of animal 1107-6 and 1706 were sent to the group of Prof. Schwinzer, MHH to verify protein expression of the complement regulatory genes using FACS (Fig. 39). These measurements could confirm CD46 and CD55 expression of animal 1107-6 as well as CD46, CD55 and CD59 expression of animal 1706. Furthermore, the function of the complement regulatory genes to protect porcine cells against human complement-mediated lysis was examined. Porcine cells were incubated for four hours with different concentrations of human serum from 5\% to 20\% (Fig. 39). Double transgenic 1107-6 cells were already better protected from lysis compared to wild type cells. 
Multi-transgenic 1706 cells were completely protected from lysis even at the highest serum concentration. Although these cells were still $\alpha$ Gal positive, the high expression levels of the complement regulatory genes could prevent complement-mediated lysis.
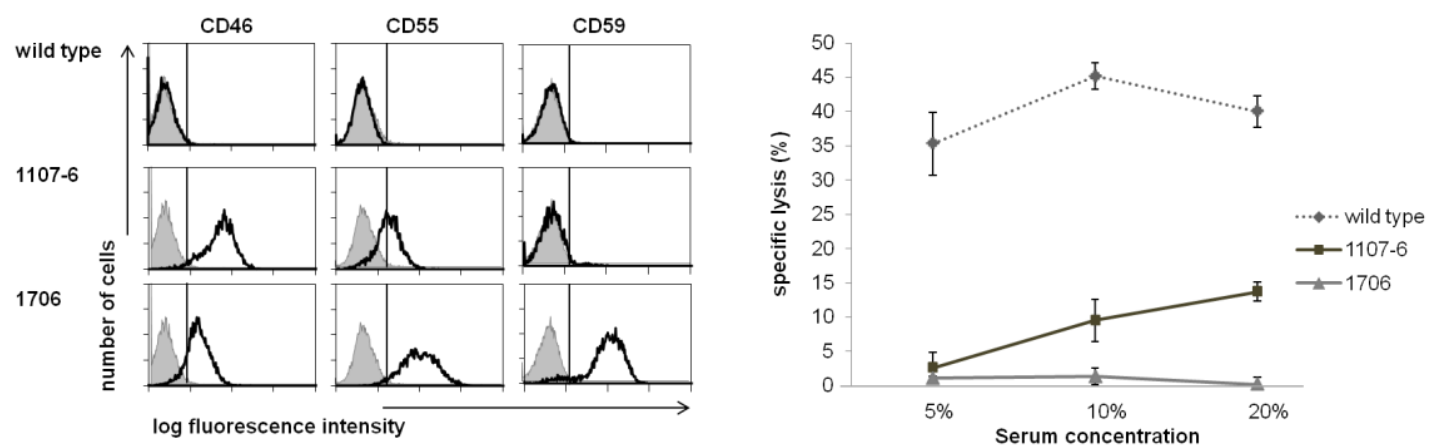

Figure 39. Left: FACS analysis of CD46, CD55 and CD59 expression in porcine kidney fibroblasts of wild type, 1107-6 and 1706 cells. Grey histograms indicate secondary antibody staining only. Right: Complement-mediated lysis assay. 51-Cr labeled kidney fibroblasts from wild type, 1107-6 and 1706 cells were incubated with various concentrations of human serum from 5\% to $20 \%$. Shown is specific lysis (mean \pm SD) calculated from triplicate samples. Data are representative of four independent experiments.

\subsubsection{GGTA1 knockout in 1706 cells}

As animal 1706 showed expression of all complement regulatory genes and complete protection of cells in the complement-mediated lysis assay, this animal was chosen for further genetic manipulation to improve xenograft protection. Kidney fibroblasts of animal 1706 were subsequently modified by a knockout of the GGTA1 gene, performed by the group of Prof. Niemann (FLI), using zinc finger nucleases (ZFN) targeting exon 8 of the GGTA1 gene as previously described (Hauschild et al., 2011). Cells containing a knockout of the GGTA1 were counterselected using isolectin B4 and magnetic beads. GGTA1-deficient 1706 cells were used for SCNT. 383 reconstructed embryos were transferred to four recipient sows, two pregnancies established, one of which continued to term and two liveborn piglets (779 and 780) and one stillborn piglet were obtained. GGTA1 sequence analysis could verify that both animals carried a one bp and a five $\mathrm{pb}$ deletion within the ZFN target region in each allele. 
Animal 780 is currently being used for breeding to found a pig line. Animal 779 was euthanized after three months due to polyarthritis. Kidney fibroblasts and porcine aortic endothelial cells (PAEC) from animal 779 were isolated and organ samples collected. RT-PCR analysis of the organ samples revealed CD46, CD55, CD59 and A20 expression in all organs analyzed. H01 expression could be verified at higher levels in heart, skin and muscle, present at lower levels in liver, kidney, spleen and PAEC. In lung no H01 expression was detectable (Fig 40).

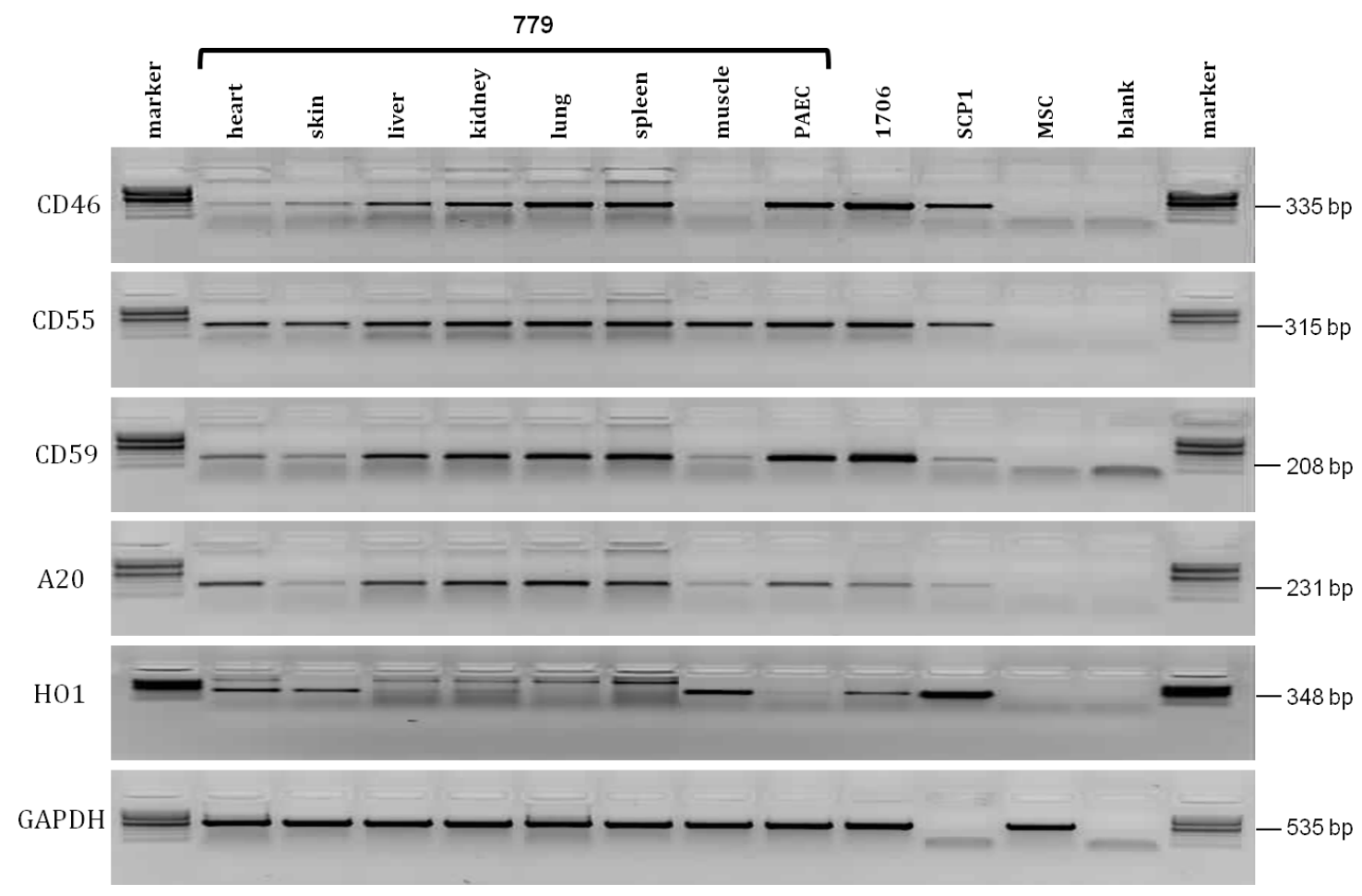

Figure 40. RT-PCT analysis of organ samples and cultured aortic endothelial cells from animal 779. Expression of kidney fibroblasts from animal 1706, expression from human SCP1 cells and wild type porcine MSCs are shown for comparison. The GAPDH primer combination used was specific for porcine GAPDH and does not amplify human GAPDH.

The RNA splicing variants of CD46 and CD55 which encode different membrane bound and soluble protein isoforms, were tested in kidney fibroblasts of animal 1706 and 779 as these protein isoforms are thought to be required for full biological activity (Tress et al., 2007; Liszewski et al., 1996). CD46 showed amplification of a $760 \mathrm{bp}$ fragment (consistent with expression of splice variants CD46-002 and CD46-004 according to Ensembl database) and a 805 bp fragment (consistent with expression of splice variants CD46-001 and CD46-007 according to Ensembl database) (Fig. 41). 
CD55 showed amplification of a large number of splicing variants, as observed in normal human tissues (Osuka et al., 2006), encoding the membrane bound protein isoforms gDAF, vDAF4 and vDAF5 and the soluble protein isoforms sDAF, vDAF1, vDAF2 and vDAF3 (names according to Osuka et al., 2006) (Fig. 41). These results show that splicing of the genomic BAC constructs of CD46 and CD55 is also functional in transgenic animals. The splice variants of CD59 were not tested as all CD59 splice variants encode the same 128 aa protein isoform (Ensembl database).

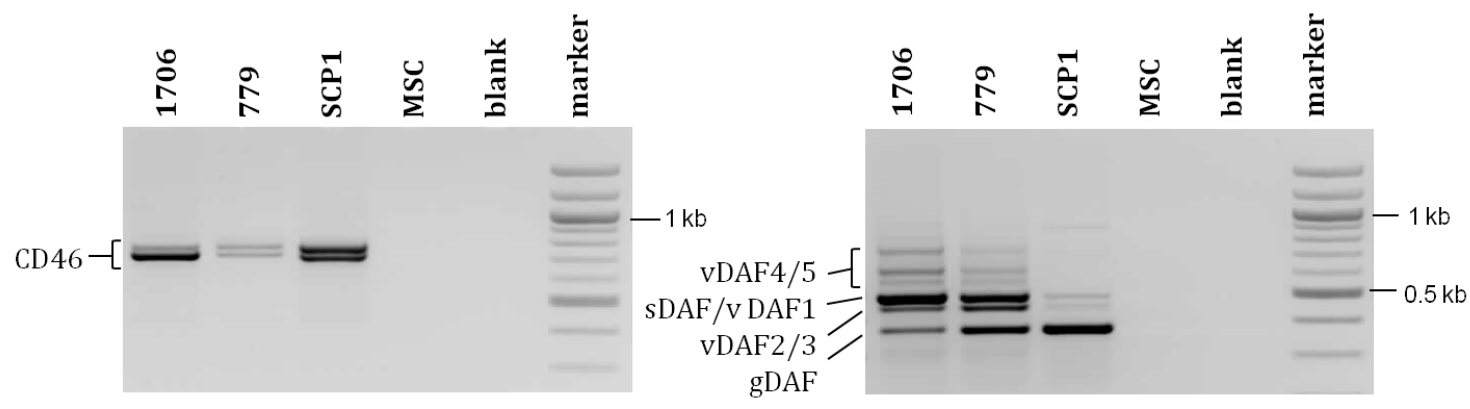

Figure 41. RT-PCR analysis verifying expression of CD46 and CD55 RNA splicing variants. Left: Expression of CD46 splicing variants. Right: Expression of CD55 splicing variants named according to Osuka et al., 2006. RNA encoding the membrane bound CD55 protein isoforms gDAF, vDAF4 and vDAF5 as well as the soluble isoforms SDAF, vDAF1, vDAF2 and vDAF3 were observed in the transgenic pigs 1706 and 779.

FACS analysis of blood samples from animals 779 and 780 (Fig. 42) revealed a homozygous GTTA1 knockout and expression of all complement regulatory genes. A complement-mediated lysis assay using kidney fibroblasts of animal 779 (Fig. 43) revealed similar results to those of 1706 cells indicating that high expression levels of the complement regulatory proteins already provide a high degree of protection from lysis in vitro. The FACS measurements and the lysis assay were conducted by the group of Prof. Schwinzer, MHH. 


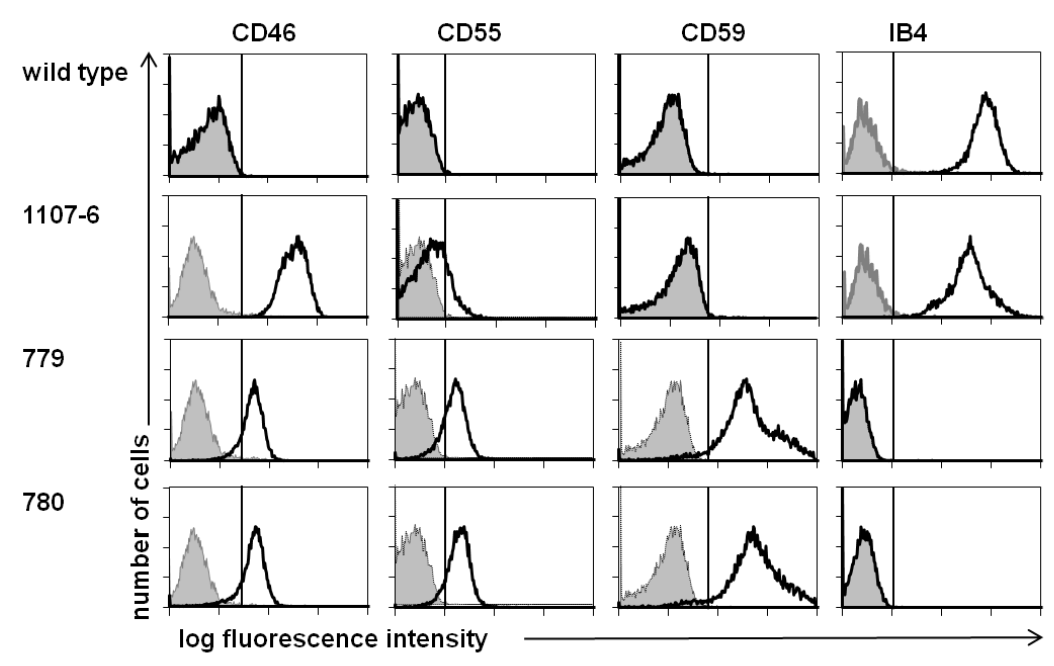

Figure 42. Flow cytometry analysis of porcine peripheral blood lymphocytes from a wild type animal, animals 1107-6, 779 and 780. Expression of human CD46, CD55 and CD59 was determined on gated lymphocytes, as determined by forward- and side-scatter characteristics. Lack of isolectin B4 (IB4) binding confirmed absence of $\alpha$ Gal epitopes in GGTA1 knockout cells of animals 779 and 780.
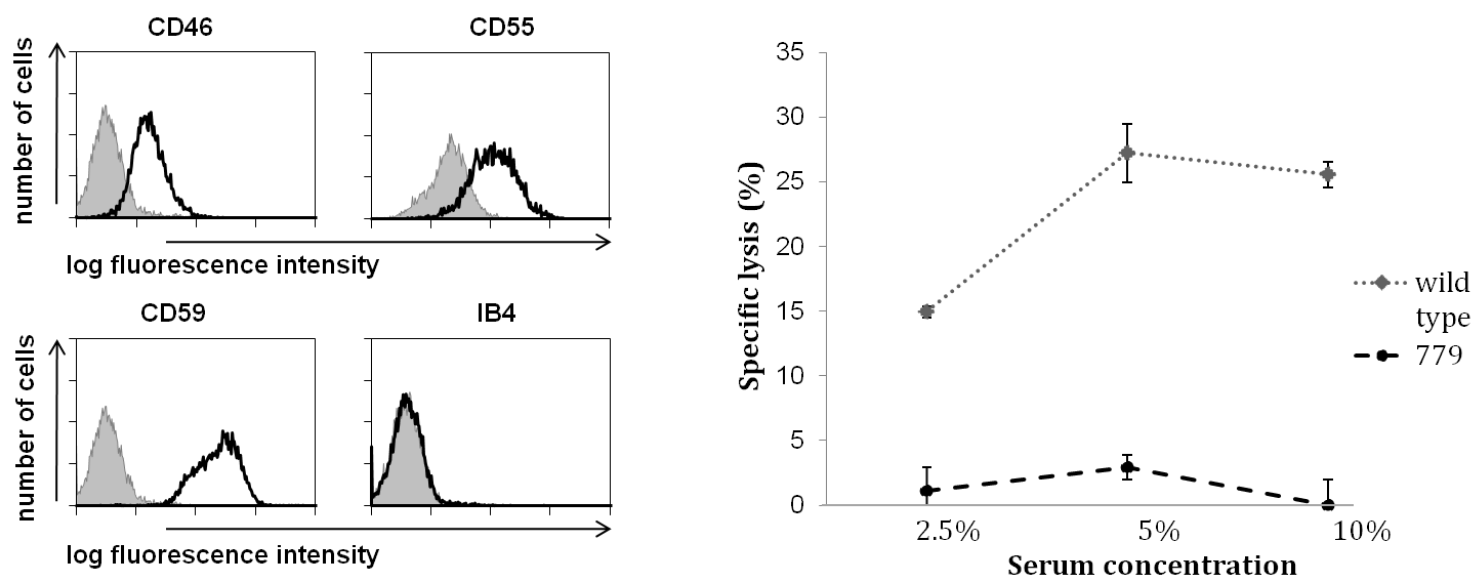

Figure 43. Functional analysis of wild type and GTTA1 knockout kidney fibroblasts from animal 779. Left: Flow cytometry analysis of human CD46, CD55 and CD59 expression. Lack of isolectin B4 (IB4) binding confirmed absence of $\alpha$ Gal epitopes. Gray histograms represent secondary antibody staining only. Right: Complement-mediated lysis assay. 51-Cr labeled kidney fibroblasts from wild type and 779 cells were incubated with various concentrations of human serum from $2.5 \%$ to $10 \%$. Shown is specific lysis (mean \pm SD) calculated from triplicate samples of one representative experiment. Similar results were obtained in a second experiment. 
The functional effects of human A20 and HO1 expression were tested on cytokine-induced upregulation of adhesion molecules in porcine aortic endothelial cells of animal 779. Human A20 has anti-apoptotic effects and inhibits the NFKB-mediated expression of E-selectin (CD62e). This effect on Eselectin expression was measured by treating the cells of animal 779 with TNF $\alpha$, revealing a reduced upregulation by $69 \%$ or $58 \%$ compared to wild type cells in the two experiments conducted (Fig. 44). Human HO1 inhibits the IFN $\gamma$ mediated upregulation of MHC class-II molecules. The expression of porcine MHC class-II molecules was tested by treatment with $8 \mathrm{ng} / \mathrm{ml}$ and $50 \mathrm{ng} / \mathrm{ml} \mathrm{IFN} \gamma$ and shown to be $44 \%$ lower compared to wild type cells (Fig. 44). The measurements were conducted by the group of Prof. Schwinzer, MHH.
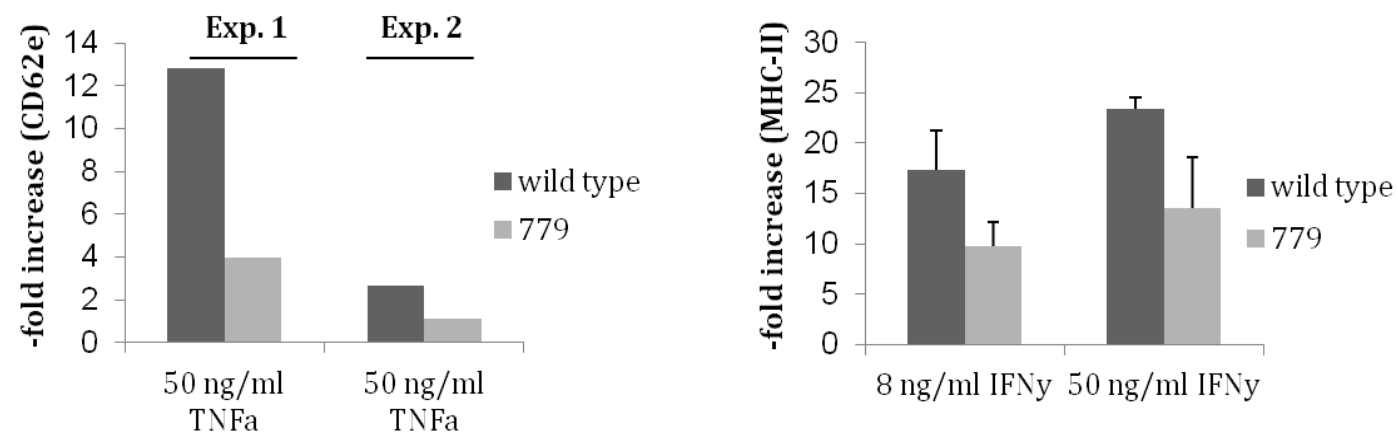

Figure 44. Functional effects of A20 and HO1 expression in cells of animal 779. Left: Effects of A20 to inhibit TNFa induced upregulation of E-selectin (CD62e). Right: Effects of HO1 to inhibit IFN $\gamma$ induced upregulation of MHC class-II molecules (Mean $+\mathrm{SD}, \mathrm{N}=3$ ).

In summary, multi-transgenic animals were made by cotransfection of BAC and PAC constructs expressing either CD46 and CD55 (pig 1107-6) or all three complement regulatory genes, CD46, CD55 and CD59 plus the anti-apoptotic and anti-inflammatory genes A20 and HO1 (pig 1706). Cells of pig 1706 were subsequently further modified by a knockout of the GGTA1 gene. Furthermore, cells and tissues of pig 1706 were precisely characterized. Determination of the transgene integration site, transgene expression, splicing of the genomic complement regulatory genes, quantification on RNA and protein level as well as functional analysis of the transgenes were performed by several in vitro assays. 


\subsection{Xeno-gene placement at the porcine ROSA26 locus}

This section no longer focuses on the usage of genomic BAC or PAC constructs but describes a further means of combining several xenograft-protective genes at one genomic locus using gene targeting. The murine Rosa26 locus is widely used for several targeting approaches. This locus encodes a ubiquitously expressed but non-functional gene that can be disrupted with no effects on physiology or development. For gene placement at the porcine ROSA26 locus several constructs were prepared that are suitable for targeted gene placement due to their small size.

\subsubsection{ROSA26 targeting and the generation of cloned piglets}

The properties of the porcine ROSA26 locus, particularly its ability to support ubiquitous transgene expression were analyzed by members of the chair (Li et al., 2014). The aim of my project was to target the porcine ROSA26 locus using a xeno-gene and to identify characteristics comparable to the murine Rosa26 locus. For the gene placement approach, an already existing ROSA26 targeting vector (ROSA26-pdx-cre-targeting vector supplied by Tatiana Flisikowska) was modified as shown in figure 45.

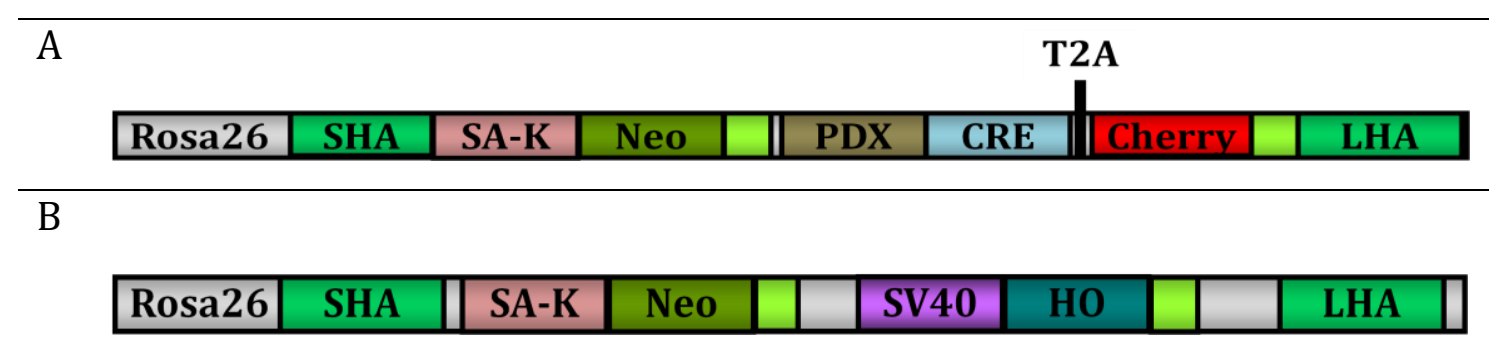

Figure 45. A: ROSA26-pdx-cre targeting vector consisting of a $2.2 \mathrm{~kb}$ short homology arm (SHA), a floxed splice acceptor kozak-neo cassette (SA-K) with a triple polyA, a pdx promoter driven creT2A-cherry cassette with a poly A and a $4.7 \mathrm{~kb}$ long homology arm (LHA). B: XhoI, SgrAI digestion of ROSA26-pdx-cre and ligation with the SV40-HO cassette cut out of pTSG1-HO1 (BglII, EcoRI digested) resulted in the ROSA26-HO-targeting vector. The total size of the construct for recombineering is about $8.8 \mathrm{~kb}$. Green boxes represent polyA cassettes. 
The ROSA26-HO-targeting vector has a $2.2 \mathrm{~kb}$ short homology arm and a $4.7 \mathrm{~kb}$ long homology arm. It is a promoter trap vector. After insertion by homologous recombination, the neomycin resistance gene is driven by the endogenous ROSA26 promoter. The H01 cDNA construct is driven by a SV40 promoter.

ROSA26-HO-targeting vector DNA was used for the electroporation of adMSC 291111 and adMSC 0102. Cells were selected using G418 until cell clones were visible. These clones were picked and further expanded. To verify a successful gene placement, genomic DNA was isolated from cell clones and screened using different primer combinations (Fig. 46). The primer combinations ROSA26 I1F2/HMOX NR (5.6 kb) and ROSA26 I1F2/ROSA26 Loc2R (3.4 kb) were used to verify a successful gene placement approach. The endogenous control PCR was conducted using the primer combination ROSA26 I1F2/ROSA26 I1R3 (3.1 kb). The targeting efficiency was about 5\%. All clones were heterozygously targeted. Correct splicing on RNA level was verified using the primer combinations SROSA26 R1/ROSA26 Loc2R (973 bp) and SROSA26 R1/ROSA26 Loc3R (1080 $\mathrm{bp}$ ), which confirmed a correct splicing from exon 1 and the splice acceptorkozak-neomycin sequence. Expression of HO1 was checked using the primer combination HMOX NF/NR (791 bp) and the PCR fragment was subsequently sequenced verifying expression of the xenogene.

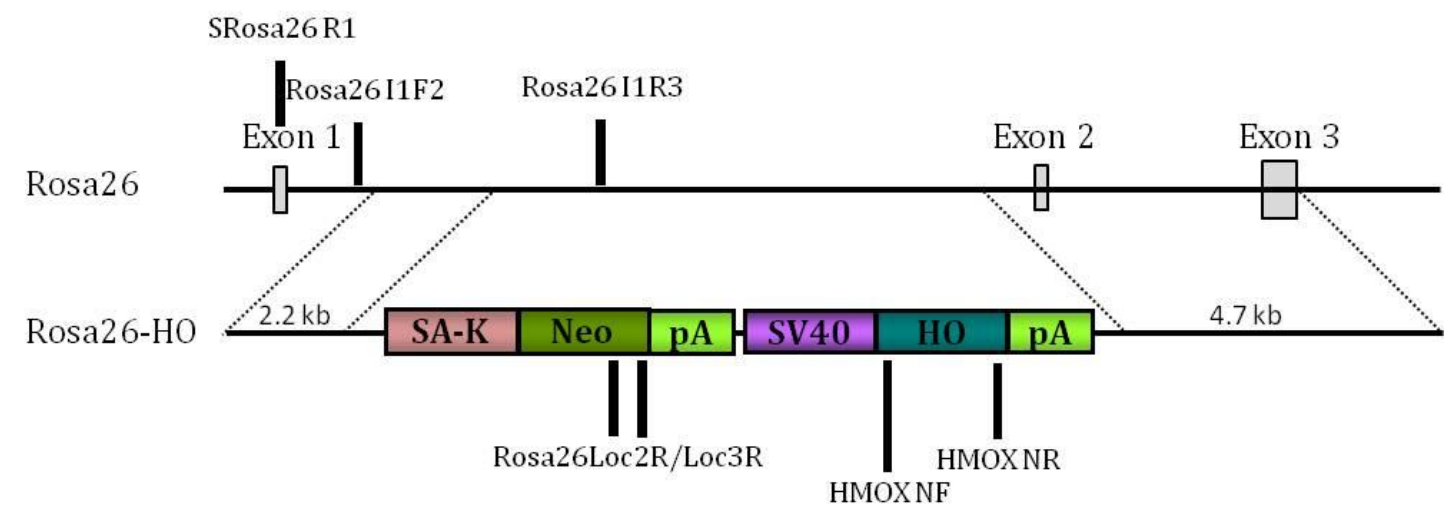

Figure 46. Targeting of the porcine ROSA26 locus and overview of the different primer combinations used. 
Positive clones were pooled and used for somatic cell nuclear transfer, conducted by the group of Prof. Wolf, LMU. 177 reconstructed embryos were transferred to two recipient sows. One pregnancy could be established and one piget was born (\#74). This piglet was further analyzed. Targeting PCRs as well as RT-PCRs were repeated using ear clip fibroblasts as described above. To confirm H01 expression on protein level, these fibroblasts were cultivated on microscope slides and used for immunofluorescence. Sections of the ear clip were also used for immunohistochemistry (Fig. 47). Due to an injury of the backbone, pig \#74 had to be euthanized and tissue samples were collected. Moreover, kidney fibroblasts were isolated and a western blot was performed to confirm HO1 expression on protein level (Fig. 48). The Santa Cruz sc-136961 mouse monoclonal IgG1 HO1 antibody (D-8) was used as primary antibody.

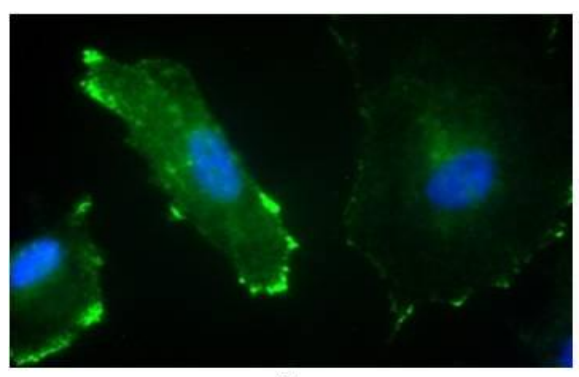

A

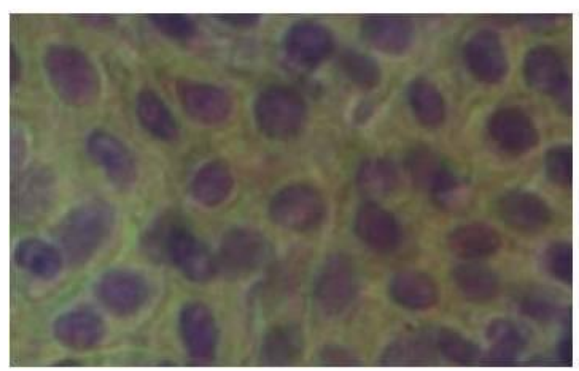

C

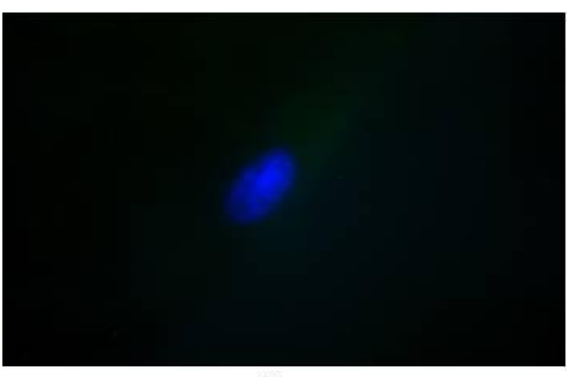

B

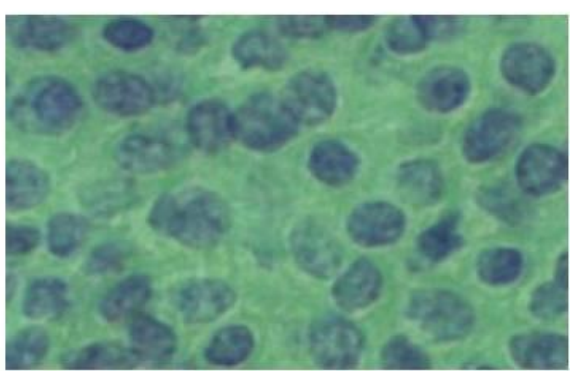

$\mathrm{D}$

Figure 47. A: Immunofluorescence of ROSA26 targeted HO1 transgenic pig \#74 showing HO1 expression in ear clip fibroblasts. A FITC-labeled secondary antibody was used for the detection of the HO1 specific primary antibody. Green color confirms HO1 expression. B: Wild type ear clip fibroblasts as negative control. C: Immunohistochemistry of ear clip sections of ROSA26-targeted HO1 transgenic piglet. A HRP-labeled secondary antibody was used for the detection of the HO1 specific primary antibody. Brown color indicates HO1 expression. D: Wild type ear clip sections as negative control. 


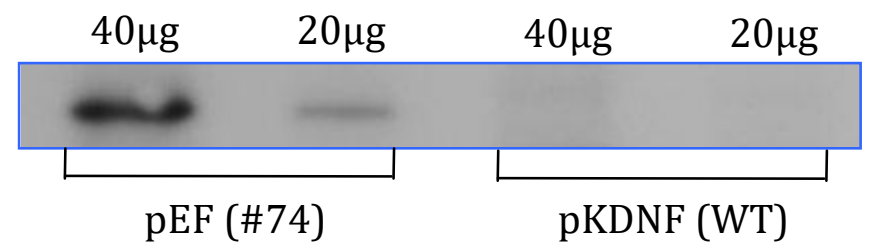

Figure 48. Western blot analysis of ROSA26 targeted HO1 transgenic pig \#74 and wild type control using $40 \mu \mathrm{g}$ and $20 \mu \mathrm{g}$ protein.

To confirm ubiquitous transgene expression, which is another important aspect of the ROSA26 locus, tissue samples of the euthanized pig \#74 were collected, RNA was isolated and HO1 expression was determined by RT-PCR using the primer combinations hHOEx1F1-HMOX3cR, delivering a band of 348 bp (Fig. 49) which was subsequently sequenced. HO1 expression could be detected in all examined tissues.
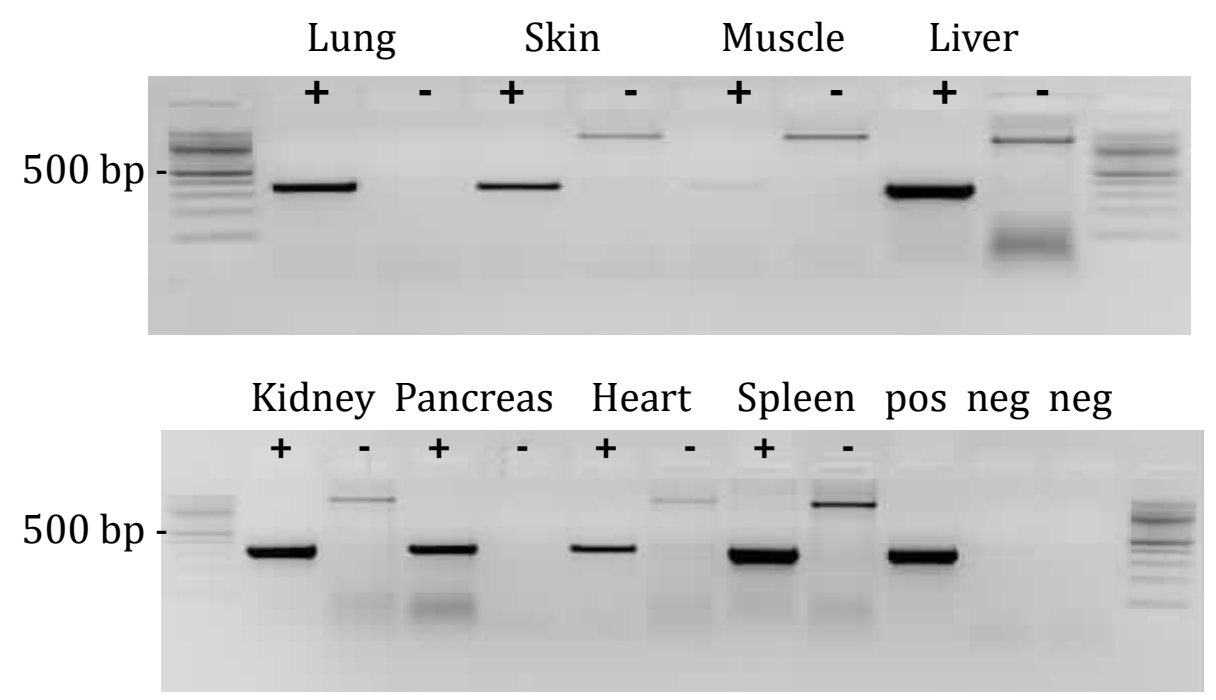

Figure 49. Tissue specific HO1 expression of the ROSA26 targeted transgenic pig \#74 and negative tissue controls of wild type animals. Positive control: SCP1. First negative control: adMSC. Second negative control: water. In muscle tissue only weak expression of HO1 could be detected due to clogging of the columns used for RNA isolation which resulted in a minor RNA quality and a low amount of RNA. The tissues of wild type animals used as negative controls delivered a non-specific band. 
As targeting of the ROSA26 locus was successful with high targeting efficiencies and resulting in xeno-gene expression, the next step was the placement of a 2nd transgene at the already targeted ROSA26-HO locus. A retargeting vector was constructed that allowed placement of the CAG-CD55 minigene in front of the SV40-HO cassette.

Figure 50 shows the retargeting approach of the ROSA26-HO locus and cloning of the retargeting vector. The cell culture experiments were carried on by Beate Rieblinger and revealed a successful retargeting of the Rosa26-HO locus using this retargeting construct.

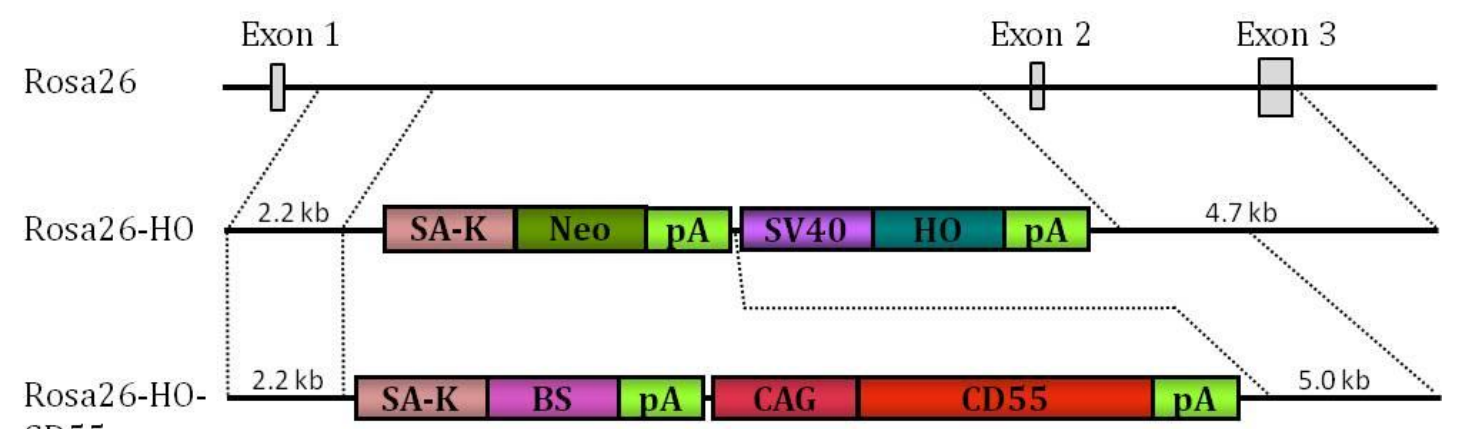
CD55

Figure 50. Retargeting approach of the ROSA26-HO locus using a CAG-CD55 minigene and exchange of the neomycin cassette by a BS cassette. The total length of the ROSA26-HO targeting construct is about $11.4 \mathrm{~kb}$ and the ROSA26-HO-CD55 retargeting vector about $12.8 \mathrm{~kb}$. For this retargeting approach the original ROSA26-HO-targeting vector was BlpI/AatII digested to reduce the size of the original long homology arm by $2.7 \mathrm{~kb}$. The residual fragment of the original long homology arm together with the SV40-H01-pA cassette which also serves as a new homologous region for recombineering resulted in a new long homology arm of $5.0 \mathrm{~kb}$. Subsequently, the $4 \mathrm{~kb}$ CAG-CD55-BGHpA fragment was cut out of the pcDNA3.1-hygro(+)-CAG-CD55-Minigene+BGHpA plasmid via a SspI/AleI double digest and cloned into the XbaI linearised pBluescript LSL-BS plasmid. Additionally, the short ROSA26 homology arm and the splice acceptor site were cut out of the ROSA26-HO-targeting vector via a MluI/EcoRV digest and the $2.4 \mathrm{~kb}$ fragment was cloned into the NheI/EcoRV linearised pBluescript-CD55-LSL-BS plasmid. Finally, the pBluescript-CD55LSL-BS plasmid containing the $2.2 \mathrm{~kb}$ ROSA26 short homology arm was KpnI/SapI digested and the $8 \mathrm{~kb}$ fragment was cloned into the SalI digested $8.2 \mathrm{~kb}$ ROSA26-HO-targeting vector with the $5.0 \mathrm{~kb}$ long homology arm. Using this retargeting vector the neomycin cassette was replaced by a BS cassette. This inhibits an accumulation of different resistance cassettes and allows iterative stacking of xeno-genes at the ROSA26 locus. 


\section{Discussion}

The major aim of this thesis was to establish different methods by which several xenoprotective genes could be efficiently combined at one genomic site in a transgenic animal. Several approaches to combine transgenes either via BAC constructs, human artificial chromosomes or gene placement at the ROSA26 locus were examined. For the multi-transgene BAC constructs, a CD55 BAC was used as basis for the modifications, because of the importance of CD55 in xenoprotection and because creation of transgenic pigs expressing high CD55 levels has been very challenging so far. As most modifications of the CD46 and CD59 constructs were performed by other members of the chair, the following sections focus on CD55. They discuss the structure of the CD55 gene, the different modifications of the CD55 BAC that were performed to enable the formation of multi-transgene BAC constructs and the various approaches that have previously been tried to obtain CD55 expressing animals, including cDNA and minigene constructs.

\subsection{Modification of the CD55 BAC construct}

The CD55 BAC was chosen for the formation of triple-transgene BAC constructs (CD55-A20-HO and CD55-TM-LEA) as CD55 efficiently inhibits formation of the C3 and C5 convertases of the complement system. By creating multi-transgene constructs, theoretically the hyperacute, acute vascular and cellular rejection could be inhibited by using just one construct. Here, the initial reduction in size of the CD55 BAC, necessary for the formation of multi-transgene constructs, which might influence transcription factor binding sites, the activity of the endogenous CD55 promoter and CD55 expression levels is discussed.

The first modifications of the CD55 BAC were to reduce the size by deletions in the $5^{\prime}$ and $3^{\prime}$ flanking regions. These modifications were necessary to enable easier transfections and the generation of multi-transgene BAC constructs (the CD55-A20-H01 and the CD55-TM-LEA constructs).

I had to ensure that the deletion in the $5^{\prime}$ flanking region did not affect any regulatory elements, the CD55 promoter activity or CD55 expression. 
Dori et al., (1993) analyzed the 5' flanking region of human CD55 in detail. They detected several transcription start sites in a $10 \mathrm{bp}$ region upstream of the ATG $(+1,+2,+8,+10)$ and the initiation codon located at +87 . In the region $880 \mathrm{bp}$ upstream of the ATG, no classical TATA or CAAT boxes were found. However, they found a TTAA at -31 which might act as a TATA box variant. Several enhancer elements such as a Sp1 binding site, cAMP responsive elements, AP-1 and AP-2 elements were found between the region from -206 to -34 . In the region from -794 to -206 an enhancer element was found which was only active in EBV cells. No other regulatory elements were found in the area from -2800 to 794. In 2005, Louis et al., discovered a hypoxia-inducible element in the region from -200 to +88 with several binding sites for the hypoxia-inducible factor (HIF).

The CD55-XS construct has a residual 5' flanking region and promoter size of 10 kb. This $5^{\prime}$ flanking region could have been further reduced. However, I did not want to delete any unknown regulatory elements. Cis-acting elements can influence promoter activity even at a distance of $50 \mathrm{~kb}$ or more (Griffiths, 2000). Moreover, the flanking regions of the CD55 construct can be affected during integration into the host genome. I tried to avoid any modifications that might negatively influence CD55 expression by using large 5' and 3' flanking regions. The CD55-XS construct was transfected in MSCs and revealed expression levels comparable to the CD55-S construct, indicating that no regulatory elements were affected by the additional $18 \mathrm{~kb}$ reduction of the $5^{\prime}$ flanking region.

\subsection{Functions of CD55}

CD55 is expressed on all serum-exposed cells and inhibits the three pathways of complement activation by the decay of the C3 and C5 convertases (Medof et al., 1984). This property has led to the development of CD55 transgenic pigs for xenotransplantation. Moreover, CD55 has several other immunological functions. The membrane-bound CD55 molecule consists of 4 short consensus repeats (SCR), a heavy 0-glycosylated serine, threonine and proline (STP)-rich domain and a C-terminal GPI-anchored domain (Coyne et al., 1992). 
SCR 2 and 3 are required for the regulation of the classical pathway, SCR 2, 3 and 4 for the regulation of the alternative pathway of complement activation (Brodbeck et al., 1996), whereas SCR1 is involved in binding of CD97. CD97 is an EGF-TM7 receptor (epidermal growth factor transmembrane receptor) and has an extended extracellular region with three N-terminal EGF domains. It is expressed on granulocytes and monocytes and is rapidly upregulated on $\mathrm{T}$ and $\mathrm{B}$ cells upon activation. The CD55-CD97 interaction can promote CD4+ ${ }^{+}$cell activation, secretion of IL-10 (interleukin 10) and GM-CSF (granulocyte macrophage colony-stimulating factor) (Capasso et al., 2006).

However, the significance of this interaction is not fully understood as CD55 has been found to suppress $\mathrm{T}$ cell response to active immunization via complementdependent mechanisms (Liu et al., 2005). Moreover, several bacterial and viral pathogens such as uropathogenic E.coli, echoviruses and enteroviruses use CD55 as a receptor molecule (Lindahl et al., 2000). Some enteroviruses bind exclusively SCR 1 (Karnauchow et al., 1998), whereas echoviruses bind to SCR $2 / 3$ or SCR3/4 (Powell et al., 1999). Although CD55 is involved in the innate immune system and upregulates NK cell activity in response to bacterial pathogen activity (Tieng et al., 2002), it is able to inhibit NK cell-mediated lysis. Miyagawa et al., (2004) checked different CD55 deletion mutants and observed that wild type and delta-SCR1 are able to inhibit complement-mediated and NKmediated lysis of porcine cells. Delta-SCR2 and delta SCR3 mutants could not suppress lysis, whereas delta-SCR4 mutants could suppress complementmediated lysis but not NK cell-mediated lysis. They also showed that much higher CD55 expression is necessary to inhibit NK cell-mediated lysis compared to complement-mediated lysis.

\subsection{A wild type CD55 cDNA or delta-SCR1 cDNA mutant for the production of transgenic animals?}

As previously discussed, the SCR1 domain of CD55 serves as a receptor for several viruses. It can upregulate T-cell activation and the formation of cytokines via the interaction with CD97. To inhibit these functions, a delta-SCR1 cDNA mutant of CD55 seems to be advantageous. 
This section describes the different animals that were made using wild type cDNA or delta-SCR1 mutant cDNA of CD55 as parts of the wild type CD55 cDNA were used for the construction of the CD55 minigene in this work as discussed in the following section.

Comparing CD55 wild type cDNA expressing the membrane bound isoform to the delta-SCR1 cDNA mutant, the delta-SCR1 mutant seems to have several advantages. The SCR1 domain cannot be used by pathogens as a receptor (Lindahl et al., 2000) and the delta-SCR1 mutant has the same capacity to inhibit complement-mediated as well as NK-cell mediated cell lysis (Miyagawa et al., 2004). Moreover, the SCR1 mediated CD55-CD97 interactions, causing a CD4+ $\mathrm{T}$ cell activation would be avoided (Capasso et al., 2006). However, the different interactions of the immune system with the CD55 SCR domains are incompletely understood.

Fisicaro et al., (2011) tried to produce multi-transgenic mice by using CD55-TMCD39 or CD55-TM-CTLA4Ig-CD39 T2A-linked cDNA constructs driven by the murine $\mathrm{H}-2 \mathrm{~K}^{\mathrm{b}}$ promoter. Although all other genes expressed well in transgenic mice, no expression of CD55 could be detected. Jeong et al., (2013) made triple transgenic pigs using an encephalomyocarditis virus (EMCV)-IRES based tricistronic vector for the expression of CD55, H-transferase and CD59. In this case the CD55 wild type cDNA construct was expressed in the transgenic animals.

Miyagawa et al., (2001) tested the expression of a delta-SCR1 CD55 cDNA and a delta-SCR1 cDNA with codon usage optimized for mammals compared to a wild type CD55 cDNA in mice. They observed that the protein levels of the different delta-SCR1 mutants were higher than wild type CD55, with the highest protein levels of the delta-SCR1 CD55 cDNA with optimal codon usage. The higher protein levels were not caused by different amounts of RNA which were almost equal for all CD55 cDNA constructs. Probably the lower wild type CD55 protein levels are caused by post-transcriptional down regulation of CD55 expression due to exon 2 (coding for SCR1) (Post et al., 1990). Miyagawa suggested using the delta-SCR1 mutant for transgenic pigs. However, all transgenic pigs produced so far have been made with either wild type CD55 cDNA or minigene constructs. 


\subsection{A CD55 minigene for the production of transgenic animals}

Several animals were created using CD55 minigenes to achieve higher expression levels than using CD55 cDNA constructs. A $6.5 \mathrm{~kb}$ CD55 minigene was described by Cozzi et al., (1997) for the generation of transgenic pigs. CD55 minigene-pigs were used by Zhou et al., (2005) to generate multi-transgenic pigs that express three complement regulatory genes. Oocytes of the CD55 transgenic pigs were microinjected with human CD46 and human CD59 genomic PAC constructs. The expression of all complement regulatory genes could be verified in these transgenic animals but CD46 expression levels were quite low. Several other CD55 minigenes were produced by Murakami et al., (1999). They consisted of a $0.9 \mathrm{~kb}$ or $5.4 \mathrm{~kb}$ human CD55 sequence driven by $0.9 \mathrm{~kb}$ or $5.4 \mathrm{~kb}$ versions of the porcine CD46 promoter. These constructs were microinjected into mouse oocytes and CD55 expression could be detected in all transgenic mice. In subsequent experiments the CD55 minigenes were used by Murakami et al., (2002) to produce transgenic pigs. Living animals were only obtained with the $0.9 \mathrm{~kb}$ promoter. Expression of this CD55 minigene could be detected in all organs of the transgenic pigs.

In my project, a CD55 minigene was cloned according to Murakami et al., (1999) that corresponds to the $0.9 \mathrm{~kb}$ minigene. As promoter, the CD46 promoter was replaced by the CAG promoter, which is known to drive ubiquitous and strong gene expression.

Former experiments already used the human CD55 promoter or the murine $\mathrm{H}$ $2 \mathrm{~K}^{\mathrm{b}}$ promoter to drive CD55 expression. The human CD55 promoter provided good expression levels in transgenic mice (Cary et al., 1993) but CD55 expression in transgenic pigs varied widely (Cozzi et al., 1997). The $\mathrm{H}-2 \mathrm{~K}^{\mathrm{b}}$ promoter allowed expression of CD55 in endothelial and epithelial cells of transgenic mice (McCurry et al., 1995) but expression was very low in transgenic pigs (Byrne et al., 1997). Therefore, the CAG promoter was chosen to drive expression of the CD55 minigene. As pA signal the BGH-pA signal was selected which holds high expression levels (Azzoni et al., 2007). This modified CD55 minigene was transfected in porcine adMSC and revealed very high CD55 expression levels (up to 100 times higher expression compared to human MSC). 
As no quantification of CD55 expression levels was performed by the reports mentioned above, it is not possible to classify the various minigenes according to their CD55 expression levels. However, the quantification of CD55 expression in this work indicates that the CAG-CD55 minigene provides very high CD55 expression levels, probably higher than obtained before using other promoter/minigene constructs. Moreover, the CAG-CD55 minigene made in this work was also used for gene placement at the ROSA26-HO1 locus. Retargeted cells were used for somatic cell nuclear transfer and several pregnancies could already be established. As soon as living piglets are born, CD55 expression levels and tissue specific expression can be determined in transgenic pigs.

\subsection{The CD46 minigene}

Besides the CD55 minigene, a CD46 minigene was additionally cloned in this work as CD46 expressing animals are used at the moment in most pig-to-baboon xenotransplantation experiments. Here, the CD46 minigene was modified according to Milland et al. (1996).

In this minigene two A+T rich regions of the CD46 cDNA were modified by silent nucleotide substitutions to increase translation efficiency and CD46 protein levels. The substituted CD46 cDNA sequence was used for the construction of a CD46 minigene according to Loveland et al., (2004).

Loveland et al., made two different versions of a CD46 minigene. Minigene mg1 contained a $343 \mathrm{bp}$ human CD46 promoter, exon 1, intron 1, exon 2, intron 2 and the wt CD46 cDNA sequence without substitutions whereas minigene mg2 contained a 636 bp human CD46 promoter, silent nucleotide substitutions in exons 3, 4 and 5, a 13 bp 3' UTR and a SV40 pA. Both minigenes were transfected into $\mathrm{CHO}-\mathrm{K} 1$ cells and the mg2 minigene showed higher expression levels than the mg1 minigene. Subsequently the mg2 minigene was used for the generation of transgenic pigs. The minigene made in this work was constructed similar to the mg2 minigene of Loveland.

The differences consisted of a 947 bp human CD46 promoter instead of the 636 bp promoter version, a 6 bp 3' UTR instead of a 13 bp 3' UTR and a BGH pA instead of the SV40 pA. 
The 3' UTR length was chosen similar to Loveland et al., as Miyagawa et al., (1997) reported down-regulation of CD46 expression levels caused by the first $125 \mathrm{bp}$ of the 3' UTR. Therefore the 3' UTR was chosen as short as possible. Moreover, the SV40 pA was replaced by a BGH pA as Azzoni et al., (2007) reported that higher expression levels were obtained by a BGH pA compared to a SV40 pA. Subsequently, the minigene made in this work was transfected in adMSC and expression levels were quantified. RT-PCR and Q-RT-PCR analysis revealed the functionality of the minigene construct and high CD46 expression compared to human MSC expression levels.

As the CD46 expression levels of the mg2 minigene were not quantified by Loveland et al., (2004), a direct comparison of expression levels is not possible. However, the modifications mentioned above indicate that higher expression levels could be obtained using the CD46 minigene made here.

The mg2 minigene was already used for the generation of CD46 transgenic pigs. These pigs showed high levels of CD46 expression in all tissues with stable expression through several generations.

The transplantation of CD46 transgenic porcine kidneys into nonimmunosuppressed baboons clearly revealed a control of complement activation and an inhibition of hyperacute rejection (Loveland et al., 2004). The CD46 transgenic pigs were further modified by a homozygous GGTA1 knockout (Hara et al., 2008) and are now distributed by Revivicor Inc., Blacksburg, USA.

\subsection{The CD39 cDNA construct}

Besides regulation of the complement system, control of the acute vascular rejection and the coagulation system is necessary to inhibit graft rejection. The ectonucleotide triphosphate diphosphohydrolase-1 (ENTPD-1; CD39) is expressed on endothelium, leukocytes and platelets (Koziak et al., 1999). It converts the proinflammatory molecules ATP and ADP to AMP which is hydrolyzed by CD73 to adenosine (Kaczmarek et al., 1996). Adenosine has antiinflammatory, anti-thrombotic and cardio protective properties (Colgan et al., 2006). 
As ADP is required to maintain the expression of the activated platelet fibrinogen receptor complex, glycoprotein $\alpha_{\mathrm{II}} / \beta_{3}$, an overexpression of CD39 can inhibit blood aggregation and thrombus formation. Cai et al., (2011) used a CD39 minigene, consisting of the murine $\mathrm{H}-2 \mathrm{~K}^{\mathrm{b}}$ promoter, an intron sequence and the CD39 cDNA sequence, to produce transgenic mice. These animals were tested in an ischemia-reperfusion experiment and myocardial protective properties of CD39 could be shown. Infarct size was significantly reduced and signaling pathways could be examined. CD39 protects from ischemic injury via adenosine $A_{2 B}$ receptor engagement and phosphorylation of Akt, ERK 1/2 and GSK-3 $\beta$. The same minigene was subsequently used by Wheeler et al., (2012) to produce CD39 transgenic pigs. Protection against myocardial injury could also be confirmed in the porcine model. In this work, a CD39 cDNA construct was generated, consisting of the CAG promoter which is known to provide high and ubiquitous expression levels in pigs, the CD39 cDNA sequence and a BGH pA which also ensures high expression levels (Azzoni et al., 2007). This minigene was transfected into porcine adMSC and high expression levels of CD39 could be detected.

However, the most suitable CD39 expression levels for biological function without risking too extended bleeding times during transplantation still have to be determined.

\subsection{Immortalization of cells using hTERT}

An increase of the life span of porcine somatic cells was necessary to enable extensive analysis after microcell-mediated chromosome transfer. The technique of MMCT was used to transfer a human artificial chromosome from human into porcine cells.

As the number of cell divisions of somatic cells is very limited, they soon undergo replicative senescence which is associated with an inactivation of telomerase and subsequent shortening of telomeres (Chiu and Harley, 1997). Telomeres consist of the tandem hexameric repeats (TTAGGG) $)_{n}$ and are highly conserved. They provide genomic stability by protecting the ends of the chromosomes from degradation, interchromosomal fusion and recombination (Hackett at al., 2001). 
As DNA polymerases are not able to completely replicate the telomeric ends, shortening of telomeres occurs with each somatic cell division (Zakian, 1995). If they have reached a critical length, genomic instability causes activation of p53 and associated signal pathways, resulting in cell cycle arrest or apoptosis (Lee et al., 1998). For the elongation of telomeres, a ribonucleoprotein complex with reverse-transcriptase activity, called telomerase, is essential. It consists of a telomerase RNA unit (TR) which is the template for the synthesis of the hexameric repeats and a telomerase reverse transcriptase unit (TERT) with catalytic activity. Whereas the TR unit is expressed in all cell types, the expression of TERT is the limiting factor for telomere elongation (Vaziri and Benchimol, 1998). Various cell types from humans (Bodnar et al., 1998), mice (Mueller et al., 2000), rats and several domestic animals including cattle (Buser et al., 2006), horses, sheep, birds, dogs and pigs (Hombach-Klonisch et al., 2006) have been immortalized by the use of hTERT.

However, not in all cases the use of hTERT was sufficient. In many cases, cotransfections of hTERT and viral oncogenes such as adenoviral E1A/E1B genes, simian virus 40 large T antigen (SV40LT) or human papilloma virus 16 (HPV16) E6/E7 genes were necessary for an efficient immortalization (Cascio, 2001). These viral oncogenes interfere with the cell cycle by inhibiting $\mathrm{p} 16 / \mathrm{pRB}$ and p53 signaling (Cascio, 2001).

Porcine hepatocyte lines were immortalized by the use of SV40LT and hTERT (Pan et al., 2008) which worked also efficient for porcine fibroblasts (Oh et al., 2007). Immortalization of porcine fetal fibroblasts by using hTERT alone was shown by Moon et al., (2014). However, other groups were not able to achieve the same results using fibroblasts from miniature or domestic pigs (Oh et al., 2007).

In this work, immortalization of porcine adMSCs and bone marrow MSCs was attempted by expression of human telomerase reverse transcriptase hTERT. Viral oncogenes were not cotransfected to avoid genetic instabilities and chromosomal damage. Cells were nucleofected with the plasmid pCl-Neo-hTERT and subsequently selected. Despite a short-term effect, no successful long-term immortalization could be observed which might be due to low expression levels of hTERT or silencing effects. 


\subsection{Human artificial chromosomes and microcell mediated chromosome transfer}

Human artificial chromosomes have the capacity to carry huge genomic constructs with all regulatory elements, are stable episomal maintained and do not integrate into the host chromosomal DNA. Moreover, they can be transferred between different species. To identify positive HAC transfers after microcellmediated chromosome transfer, I attempted to immortalize somatic porcine cells. The next challenge was to generate xeno-HACs and to establish MMCT. Several attempts to obtain a human artificial chromosome containing xenogenes failed, resulting only in chromosomal integration of the transfected DNA constructs.

Cotransfections of various genomic CD46, CD55 and CD59 constructs as well as triple transgene-BAC constructs were performed together with a NotI linearized, tetra-telomerised PAC construct (pTTE1) containing $116 \mathrm{~kb}$ of human chromosome 5 alpha satellite DNA, a CMV/EGFP expression cassette and two BS resistance genes. The tetra-telomerized construct was thought to stabilize the chromosomal ends via T-loops and to result in a higher amount of HAC formation. However, the frequency of HAC formations using the pTTE1 construct was comparable to the di-telomerized constructs as shown by Laner (2004). For the delivery of the xeno-HAC components into the cell lipofection, microinjection and bactofection are possible methods. Lipofection was chosen using 10-200 ng of the pTTE1 construct per $10 \mathrm{~cm}$ plate as this small amount was published to result in the highest probability of HAC formation (Laner, 2004).

Cotransfection experiments of HAC vectors and transgenes were shown to work efficiently (Laner, 2004; Mejia et al., 2002). For example, a $100 \mathrm{~kb}$ fragment of human alpha satellite DNA derived from chromosome 21 was cotransfected with a $180 \mathrm{~kb}$ BAC construct in HT1080 cells and HAC formation was achieved in 6\% of all transgene-expressing cells. However, a huge number of random chromosomal integration was also observed (Ikeno et al., 2002). 
The general problem concerning HAC formation is their unpredictable structure and the unknown relationship between the transfected DNA and the resultant HAC composition (Ikeno et al., 2002; Mejia et al., 2002; Basu et al., 2005). De novo HACs are probably formed by concatemerization of the transfected DNA.

There are indications that the centromeric region is species specific. As the stability and functionality of human centromeric regions in porcine cells as well as interspecies centromeric protein interactions have not been investigated so far, human and porcine centromeric higher order repeats were used for the HAC formation.

In pigs, some centromeric DNA families are known. The Mc1 family consists of divergent $340 \mathrm{bp}$ monomeric repeats and is specific for the metacentric chromosomes 1-12 and the X chromosome. The Ac2 family consists of $14 \mathrm{bp}$ monomeric units and is specific for the acrocentric chromosomes 13-18. Chromosome 11 was shown to contain a 51 bp repeat (Mc2) (Janzen et al., 1998). As the Mc1 monomeric unit is the most common one in pigs and was successfully used for the formation of a porcine artificial chromosome via yeast-pig cell fusion (Poggiali et al., 2002), this family was chosen for the cotransfection experiments. However, in none of the conducted cotransfections a successful HAC formation could be detected.

After the formation of xeno-HACs in human HT1080 cells these were to be transferred into porcine ST007 cells or MSCs via MMCT.

So far, inter-species chromosome transfer was used to carry human chromosomes into mouse ES cells, chinese hamster cells or bovine fibroblasts (Tomizuka et al., 1997; Hernandez et al., 1999; Guiducci et al., 1999; Kuroiwa et al., 2002). The transfer of a HAC from a hamster cell line to a mouse ES cell line was reported by Paulis et al., (2007). However, attempts to transfer a HAC into porcine cells or a porcine minichromosome into human cells were so far not successful (Cavaliere et al., 2009). The formation of a porcine artificial chromosome was reported by Poggiali et al., (2002) via the fusion of a yeast strain, carrying a YAC with porcine centromeric DNA, and porcine cells. Attempts to transfer the porcine artificial chromosome into human, mouse or CHO cells also failed. Moreover, Nowak-Imialek et al., (2010) reported rapid loss of porcine chromosomes from mouse-pig hybrid cell lines. 
As the transfer efficiency and stability of the HAC in the recipient cell line depends very strongly on the centromere sequence and the genetic background (Vissel, Choo 1991), Cavaliere et al., (2009) compared the satellite and centromeric sequences from sheep, cattle, human, mouse and pigs. They found two groups of high sequence similarity between pig, sheep and cattle as well as mouse and humans. They verified this by the transfer of a porcine artificial chromosome into sheep cells. However, Ikeno et al., (2009) could successfully transfer a HAC, carrying $50 \mathrm{~kb}$ of human alpha satellite DNA, from human into porcine PK15 cells demonstrating that the transfer efficiency depends extremely on the HAC centromeric sequence. Here, a human artificial chromosome was successfully transferred from human HT1080 cells into porcine ST007 cells. The transfer was verified by PCR and FISH analysis. To exclude an undesired transfer of additional human chromosomes from the HT1080 cells into the porcine cells, several PCRs were conducted screening for human genes on different human chromosomes. The transfer of the HAC into porcine MSC was also successful. However, additional human chromosomes were transferred too. This could be prevented by smaller filter sizes for the microcells and a shortened fusion time using polyethylene glycol.

\subsection{ROSA26 targeting and expression of $\mathrm{HO1}$}

The murine Rosa26 locus has become the preferred site of transgene integration, as it provides ubiquitous transgene expression and disruption of the locus has no deleterious effects on physiology or development (Zambrowicz et al., 1997; Irion et al., 2007; Kobayashi et al., 2012). The porcine ROSA26 locus has previously been targeted (Li S. et al., 2014), revealing ubiquitous expression of inserted transgenes in living pigs and that the strength of the porcine ROSA26 promoter is comparable to the $\mathrm{H}-2 \mathrm{~K}^{\mathrm{b}}$ promoter (Li P. et al., 2014).

In this work, the porcine ROSA26 locus was targeted with a promoter trap vector, consisting of a $2.2 \mathrm{~kb} 5^{\prime}$ short homology arm, a splice-acceptor Neo cassette, a SV40-driven H01 gene and a 4.7 kb long 3' homology arm. The Neo resistance gene was driven by the endogenous ROSA26 promoter after insertion by homologous recombination. 
Positively targeted clones were pooled and used for nuclear transfer. One living pig (74) was obtained and targeted insertion was verified on DNA and RNA level. Expression of the HO1 gene could be detected in lung, skin, muscle, liver, kidney, pancreas, heart and spleen. H01 protein levels were checked using western blot and immunohistochemistry. Both methods verified high HO1 protein levels. The SV40-HO1 construct was also used for the generation of HO1 expressing pigs by Petersen et al., (2011). It was reported that porcine fibroblasts were electroporated with the HO1 plasmid, resulting in random integration of an unknown copy number at an unknown number of integration sites. These animals expressed HO1 in heart, kidney, liver, endothelial cells and fibroblasts. The H01 protein could not be directly detected as the available antibodies at this time showed a strong cross-reactivity. Therefore, the presence of HO1 protein was confirmed by using a H01-FLAG fusion protein assay. TNF- $\alpha$-mediated apoptosis and a reduction in the expression of adhesion molecules such as ICAM1, VCAM-1 and E-selectin was also observed. In an ex vivo perfusion experiment of porcine kidneys, the H01 transgenic kidneys could be perfused for 240 minutes compared to 60 minutes of wild type pigs.

Yeom et al., (2012) reported pigs transgenic for a randomly integrated CMV-HO1 cDNA construct which contained a hemaglutinin epitope to enable easier detection. Transgenic animals were expressing HO1 in heart, kidney, lung, liver, pancreas, spleen and skin with a huge variation in expression levels.

Although in the ROSA26-HO1 gene placement approach just a single copy of a cDNA construct was expressed, high levels of HO1 could be detected in all examined tissues. As the genomic integration site is defined, the same locus can also be used for a gene-stacking experiment to place several xeno-genes at one permissive site. The retargeting vector for the placement of a CD55 minigene adjacent to HO1 and an exchange of the resistance cassette was already prepared. This retargeting vector enabled a successful retargeting of the ROSA26-H01 locus in subsequent experiments. 


\subsection{Multi-transgenic animals}

This work describes the generation of two lines of multi-transgenic pigs expressing two and three human complement regulatory genes. Overexpression of complement regulatory genes efficiently inhibits complement activation, complement-mediated cell lysis and hyperacute xenograft rejection.

Transgenic pigs carrying single human complement regulatory genes have previously been made by several groups using cDNA constructs, minigenes or genomic constructs (Cozzi et al., 1995; Mollnes et al., 2003; Adams et al., 2001). Hearts from donor pigs transgenic for single complement regulators have survived up to 139 days as heterotopic grafts in non-human primates (Byrne et al., 2006) and up to 39 days as orthotopic transplants (Vial et al., 2000).

The combination of several complement regulatory genes has also been investigated. Xenografts from mice transgenic for human CD55 and CD59 or CD46, CD55 and CD59 clearly showed greater protection from complementmediated damage than single-transgenic animals (Cowan et al., 1998; Oldham et al., 1996). This also could be shown using double- and triple-transgenic porcine cells in in vitro experiments (Zhou et al., 2005).

However, the combinations of CD46 and CD55 or CD55 and C59 have so far not extended xenograft survival in heterotopic (Lin et al., 2000) or orthotopic pig-tobaboon xenotransplantation experiments (Brandl et al., 2007). This is almost certainly because the double transgenic pig lines available did not provide sufficient expression levels to protect the xenograft. The benefits of combining several complement regulatory genes have been shown, so there is a clear need for transgenic pigs that express high levels of two or three complement regulators.

There has been only one report of pigs transgenic for three complement regulatory genes (Zhou et al., 2005). They microinjected genomic CD46 and CD59 constructs into zygotes from a CD55 transgenic line: 884 injected embryos were transferred into 34 recipients, 154 piglets were born and one piglet expressing all three complement regulatory genes was identified. However, the piglet identified did not reveal high expression levels of all three complement regulatory genes nor ubiquitous expression. 
The approach taken in my project was to streamline the procedure and to reduce the number of animals required to derive high expressing pigs by pre-selecting high expressing cell clones prior to somatic cell nuclear transfer. The production of the multi-transgenic pig 1706, involved the transfer of 238 reconstructed embryos into two recipient sows, one pregnancy was established and one piglet was obtained. This and other pigs generated by nuclear transfer showed levels of transgene expression predicted from their parental cell clones and no nontransgenic animals were produced. The 1706 transgenic line reveals high protein levels of all complement regulatory genes as shown by FACS analysis.

It is however difficult to directly compare RNA and protein levels of the 1706 transgenic line with previous reports as few publications present quantitative data. A functional test of protection against complement-mediated lysis thus offers the most meaningful form of comparison. Jeong et al., (2013) produced and tested transgenic pigs that expressed cDNA constructs of CD55, Htransferase and CD59, driven by the CMV promoter and localized on a tricistronic expression vector. Swine umbilical vein endothelial cells from the highest expressing pigs were incubated for 6 hours with 20\% human serum. Although these animals expressed H-transferase and showed strong downregulation of $\alpha$-Gal epitopes, transgenic cells underwent about $40 \%$ lysis compared to $80 \%$ lysis of wild type. Zhou et al., (2005), described above, showed $20 \%$ lysis of porcine peripheral blood monocytes incubated with $25 \%$ human serum after one hour compared to $50 \%$ lysis of wild type.

The results described in my project show a dramatic advance over such previous findings. I report about 10\% lysis of kidney fibroblasts from the CD46 and CD55 expressing pig 1107-6 after incubation with 20\% human serum for four hours compared to $40 \%$ lysis of wild type cells. Most importantly, kidney fibroblasts from the 1706 transgenic pig were completely protected from lysis under the same conditions, an unprecedented finding. Overexpression of complement regulatory genes is designed primarily to combat hyperacute xenograft rejection. To address the next obstacle, acute vascular rejection, the anti-inflammatory and anti-apoptotic genes HO1 and A20 were also included in the 1706 multitransgene array. Acute vascular rejection is characterized by endothelial activation, inflammation, thrombus formation and xenograft rejection. 
Petersen et al., (2011) and Yeom et al., (2012) showed that H01 expression protects endothelial cells from TNF- $\alpha$-mediated apoptosis, supports prolonged organ survival of porcine kidneys in ex vivo perfusion experiments with human blood, protects porcine fibroblasts from $\mathrm{H}_{2} \mathrm{O}_{2}$ damage and reduces TNF- $\alpha$ and cycloheximide-mediated apoptosis.

H01 expression could be detected in almost all tissues of the 1706 transgenic pig and cytokine-induced MHC-II upregulation in porcine aortic endothelial cells was clearly reduced. A20 expression was found to be ubiquitous. A20 inhibits NF- $\kappa B$ activation, the upregulation of pro-inflammatory and pro-apoptotic cytokines including TNF $\alpha$ and interleukin-1 $\beta$ (Dixit et al., 2002) and has been shown to protect porcine aortic endothelial cells against apoptosis and porcine xenografts partially against ischemia/reperfusion injury (Oropeza et al., 2009).

The function of A20 in the 1706 line was verified by a strong reduction of Eselectin upregulation (about 65\%) after cytokine induction. However, it was not possible to clearly ascribe these protective effects to A20 or H01 individually, and it is possible that coexpressed A20 and HO1 act in synergy.

The multi-transgenic 1706 line thus addresses hyperacute rejection and to some extent acute vascular rejection. To provide further protection in vivo, the GGTA1 gene was subsequently inactivated. The combination of complement regulatory genes with GGTA1 knockout has already shown to very efficiently prolong xenograft survival (Van Denderen et al., 1997; Cowan et al., 1998; McGregor et al., 2012). To facilitate transplantation into human patients, the gene $C M A H$, responsible for the major non-Gal antigen Neu5Gc will be inactivated. Expression of additional transgenes will probably be necessary to further reduce acute vascular and cellular rejection. The 1706 multi-transgene combination should also have protective effects at these levels. High CD55 expression levels have already been shown to inhibit NK cell activation (Miyagawa et al., 2004). CD46 expression has significant effects on inhibition of procoagulant and antifibrinolytic pathways (Bongoni et al., 2015) and a combination of GGTA1 knockout and CD46 expression was shown to down-regulate the human T-cell response to porcine cells (Ezzelarab et al., 2011; Wilhite et al., 2012). A20 expression also inhibits T-cell activation (Coornaert et al., 2008). 
The precise requirements for additional transgenes to achieve long-term xenograft survival have still to be determined.

\section{Summary and outlook}

So far, progress has been made to overcome the hyperacute and to some extent the acute vascular rejection. The new challenge is to combine the different transgenes and knockouts efficiently in one pig to enable breeding of the animals and a continuous supply of donor organs.

Achieving this was the focus of the work presented here. Several transgenes can either be combined on a single artificial chromosome or can be colocalized at the same genomic locus. The latter can be achieved by using large BAC constructs carrying several xenogenes. This enables the co-integration of all transgenes after transfection at the same genomic site but without the possibility of any targeted integration. The second approach consists of a repeated targeting of the porcine ROSA26 locus. This way, the genomic integration site is known and precise gene-stacking is possible. Both approaches can be performed in primary porcine adMSC, enabling the selection of stable, high expressing cell clones which can be used for somatic cell nuclear transfer to obtain transgenic animals.

The recent development of very efficient and cheap genome editing tools, such as the CRISPR/Cas9 system facilitates the inactivation of porcine genes such as GGTA1 or CMAH. If required, these animals can be subsequently further modified. Transgenic animals have to be kept at defined pathogen-free (DPF) conditions which enable elimination of most microorganisms. Genetically modified pigs can be transferred into these DPF facilities via caesarean sections or gnotobiotic isolators.

However, porcine endogenous retroviruses (PERVs) remain a problem but screening and selection of low expressing animals or PERV-C free animals is possible. Moreover, screening for relevant microorganisms including viruses has been established allowing monitoring of almost all known potential threats. Despite all porcine genetic modifications, immunosuppression for the patient will still be necessary in the near future. However, the probability for xenograft rejection and the level of immunosuppression can be dramatically reduced by using multi-modified transgenic pigs. 


\section{Abbreviations}

\begin{tabular}{|c|c|}
\hline A20 & Tumor necrosis factor $\alpha$ induced protein 3 (human) \\
\hline adMSC & Adipose tissue derived MSC \\
\hline AHXR & Acute humoral xenograft rejection \\
\hline APC & Antigen presenting cell \\
\hline AVR & Acute vascular rejection \\
\hline BAC & Bacterial artificial chromosome \\
\hline BGH & Bovine growth hormone \\
\hline CAG & CMV enhancer/chicken $\beta$-actin/rabbit globin (promoter) \\
\hline CCR5 & Chemokine receptor type 5 \\
\hline CD39 & $\begin{array}{l}\text { Ectonucleotide triphosphate diphosphohydrolase-1; ENTPD-1 } \\
\text { (human) }\end{array}$ \\
\hline CD46 & Membrane cofactor protein, MCP (human) \\
\hline CD55 & Decay-accelerating factor, DAF (human) \\
\hline CD59 & Protectin (human) \\
\hline CD62e & E-selectin \\
\hline CMAH & $\begin{array}{l}\text { Cytidine monophosphate- } \mathrm{N} \text {-acetylneuraminic acid } \\
\text { hydroxylase }\end{array}$ \\
\hline CRISPR & Clustered regularly interspaced short palindromic repeat \\
\hline CRP & Complement regulatory protein \\
\hline CTLA4 & Cytotoxic T lymphocyte-associated antigen 4 (human) \\
\hline ES cells & Embryonic stem cells \\
\hline FACS & Fluorescence-activated cell sorting \\
\hline FISH & Fluorescence in situ hybridization \\
\hline GPI & Glycosyl-phosphatidylinositol \\
\hline GGTA1 & $\alpha 1,3$-galactosyltransferase gene \\
\hline HAC & Human artificial chromosome \\
\hline HAR & Hyperacute rejection \\
\hline HBXIP & Hepatitis B X-interacting protein \\
\hline $\mathrm{H}-\mathrm{D}$ antigens & Hanganutziu-Deicher antigens \\
\hline HO1 & Heme oxygenase 1 (human) \\
\hline
\end{tabular}




\begin{tabular}{|c|c|}
\hline HR & Homologous recombination \\
\hline hTERT & Human telomerase reverse transcriptase \\
\hline H-transferase & Human $\alpha 1,2$-fucosyltransferase \\
\hline ICAM-1 & Intercellular adhesion molecule 1 \\
\hline IL-10 & Interleukin 10 \\
\hline IL-1 $\beta$ & Interleukin 1 beta \\
\hline IFN- $\gamma$ & Interferon $\gamma$ \\
\hline LEA29Y & $\begin{array}{l}\text { High affinity variant of cytotoxic T-lymphocyte antigen } 4 \\
\text { immunoglobulin (CTLA4Ig); L104E and A29Y substitution }\end{array}$ \\
\hline MMCT & Microcell-mediated chromosome transfer \\
\hline Neu5Ac & $\mathrm{N}$-acetylneuraminic acid \\
\hline Neu5Gc & N-glycolylneuraminic acid \\
\hline NHEJ & Non-homologous end joining \\
\hline NK & Natural killer \\
\hline NKG2D & Natural-killer group 2 member D \\
\hline NLS & Nuclear localization sequence \\
\hline $\mathrm{pA}$ & Poly adenylation \\
\hline PAC & Phage artificial chromosomes \\
\hline PAEC & Porcine aortic endothelial cells \\
\hline PCR & Polymerase chain reaction \\
\hline PERV & Porcine endogenous retrovirus \\
\hline PFGE & Pulsed-field gel electrophoresis \\
\hline pTTE1 & Tetra-telomerised PAC construct \\
\hline RIP & Receptor interacting protein \\
\hline SCNT & Somatic cell nuclear transfer \\
\hline SCR & Short consensus repeat \\
\hline $\operatorname{SIRP} \alpha$ & Signal-regulatory protein- $\alpha$ \\
\hline SLA & $\begin{array}{l}\text { Swine leukocyte antigen; porcine major histocompatibility } \\
\text { complex class I }\end{array}$ \\
\hline SOE-PCR & Splice overlap extension PCR \\
\hline STP & Serine, threonine and proline \\
\hline TAFI & Thrombin-activatable fibrinolysis inhibitor \\
\hline
\end{tabular}




\begin{tabular}{ll}
\hline TALEN & Transcription activator-like effector nuclease \\
\hline TFPI & Tissue factor pathway inhibitor \\
\hline TGF- $\beta$ & Transforming growth factor $\beta$ \\
\hline TNF- $\alpha$ & Tumor necrosis factor $\alpha$ \\
\hline TM & Thrombomodulin (human) \\
\hline TRAF & TNF-receptor-associated factor \\
\hline ULBP1 & UL16-binding protein 1 \\
\hline UTR & Untranslated region \\
\hline ZFN & Zinc-finger nuclease \\
\hline
\end{tabular}

\section{List of Tables}

\begin{tabular}{llc}
\hline Table 1 & Analytical digest & 48 \\
\hline Table 2 & Blunting digest & 49 \\
\hline Table 3 & PCR settings & 51 \\
\hline Table 4 & Nick translation & 55 \\
\hline Table 5 & Probes for the detection of xeno-genes & 56 \\
\hline Table 6 & Primer sequences for the SOE-PCR & 82 \\
\hline
\end{tabular}

\section{List of Figures}

\begin{tabular}{lll}
\hline Figure 1 & Hyperacute rejection after xenotransplantation & 12 \\
\hline Figure 2 & Acute vascular rejection & 13 \\
\hline Figure 3 & Pathways of the complement system & 14 \\
\hline Figure 4 & Pathways of blood coagulation & 19 \\
\hline Figure 5 & Biological functions of H01 & 21 \\
\hline Figure 6 & Functions of A20 & 23 \\
\hline Figure 7 & Cellular rejection mechanisms & 25 \\
\hline Figure 8 & Schematic diagram for cloning the plasmid PL452-spec & 69 \\
\hline Figure 9 & Schematic diagram for cloning the plasmid PL452inf-BSinf & 69 \\
\hline
\end{tabular}




\begin{tabular}{|c|c|c|}
\hline Figure 10 & Schematic diagram of CD46 BAC/CD59 PAC constructs & 70 \\
\hline Figure 11 & Schematic diagram of the CD55 BAC construct & 71 \\
\hline Figure 12 & Schematic diagram of the CD55-XS construct & 72 \\
\hline Figure 13 & Schematic diagram of the CD55-S-TM construct & 74 \\
\hline Figure 14 & Schematic diagram of the CD55-XS-TM-LEA construct & 75 \\
\hline Figure 15 & RT-PCR analysis of the CD55-XS-TM-LEA construct & 76 \\
\hline Figure 16 & Schematic diagram of the CAG-CD55-A20-HO1 construct & 77 \\
\hline Figure 17 & RT-PCR analysis of different CD55 splicing variants & 78 \\
\hline Figure 18 & The CAG-CD55 minigene & 80 \\
\hline Figure 19 & Quantification of CAG-CD55 expression & 81 \\
\hline Figure 20 & Structure of the CD46 minigene & 83 \\
\hline Figure 21 & Quantification of CD46 expression levels & 84 \\
\hline Figure 22 & Quantification of CD39 expression & 85 \\
\hline Figure 23 & RT-PCR analysis of HBXIP expression & 87 \\
\hline Figure 24 & Quantification of porcine CD55 in HBXIP expressing cells & 87 \\
\hline Figure 25 & RT-PCR expression analysis of hTERT expression & 89 \\
\hline Figure 26 & FISH analysis of the HT1080 cell line EHG6k & 91 \\
\hline Figure 27 & Transient EGFP expression of porcine MSC after MMCT & 92 \\
\hline Figure 28 & Metaphase chromosome spread of MSC after MMCT & 93 \\
\hline Figure 29 & Metaphase chromosome spreads of ST007 after MMCT & 94 \\
\hline Figure 30 & Tetra-telomeric (Tel) pTTE1 vector & 95 \\
\hline Figure 31 & RT-PCR analysis of GGTA1 knockout clone C1F6-2 & 97 \\
\hline Figure 32 & RT-PCR analysis of clone 3-6 & 98 \\
\hline Figure 33 & RT-PCR analysis of clone 4-39 & 98 \\
\hline Figure 34 & RT-PCR analysis of tissues of animal 1107-6 & 99 \\
\hline Figure 35 & RT-PCR analysis of tissues of animal 1706 & 100 \\
\hline Figure 36 & Quantification of expression levels in tissues of pig 1706 & 100 \\
\hline Figure 37 & Metaphase chromosome spreads of animal 1706 & 101 \\
\hline Figure 38 & Metaphase chromosome spreads and Q-banding & 101 \\
\hline Figure 39 & FACS analysis and lysis assay of pigs $1107-6$ and 1706 & 102 \\
\hline Figure 40 & RT-PCT analysis of organ samples of animal 779 & 103 \\
\hline Figure 41 & RT-PCR analysis of CD46 and CD55 splicing & 104 \\
\hline
\end{tabular}




\begin{tabular}{lll}
\hline Figure 42 & FACS analysis and lysis assay of pigs 1107-6, 779 and 780 & 105 \\
\hline Figure 43 & Functional analysis of kidney fibroblasts from animal 779 & 105 \\
\hline Figure 44 & Functional effects of A20 and HO1 expression of pig 779 & 106 \\
\hline Figure 45 & ROSA26 targeting vector & 107 \\
\hline Figure 46 & Targeting of the porcine ROSA26 locus & 108 \\
\hline Figure 47 & Immunofluorescence of ROSA26 targeted H01 pig 74 & 109 \\
\hline Figure 48 & Western blot analysis of ROSA26 targeted H01 pig 74 & 110 \\
\hline Figure 49 & Tissue specific H01 expression of pig 74 & 110 \\
\hline Figure 50 & Retargeting approach of the ROSA26-HO locus & 111 \\
\hline
\end{tabular}

\section{Literature}

Abeyama K, Stern D, Ito Y, et al. The N-terminal domain of thrombomodulin sequesters high-mobility group-B1 protein, a novel anti-inflammatory mechanism. J Clin Invest 2005; 115:1267-1274.

Adams DH, Kadner A, Chen RH, et al. Human membrane cofactor protein (MCP, CD46) protects transgenic pig hearts from hyperacute rejection in primates. Xenotransplantation 2001;8:36.

Ahmad SR, Lidington EA, Ohta R, et al. Decay-accelerating factor induction by tumour necrosis factor- $\alpha$, through a phosphatidylinositol-3 kinase and protein kinase C-dependent pathway, protects murine vascular endothelial cells against complement deposition. Immunology 2003;110:258-268.

Amara U, Flierl MA, Rittirsch D, et al. Molecular Intercommunication between the Complement and Coagulation System. J Immunol 2010;185(9): 5628-5636.

Amor D, Bentley K, Ryan J, et al. Human centromere repositioning "in progress". Proc Natl Acad Sci USA 2004;101:6542-6547.

Azzoni AR, Ribeiro SC, Monteiro GA, et al. The impact of polyadenylation signals on plasmid nuclease resistance and transgene expression. Journal of Gene Medicine 2007;9(5):392-402.

Bardor M, Nguyne DH, Diaz S, et al. Mechanism of uptake and incorporation of the non-human sialic acid N-glycolylneuraminic acid into human cells. J Biol Chem 2005;280,4228-4237.

Barnett MA, Buckle VJ, Evans EP, et al. Telomere directed fragmentation of mammalian chromosomes. Nucleic Acids Res 1993;21:27-36. 
Bartosch B, Stefanidis D, Myers R, et al. Evidence and consequence of porcine endogenous retrovirus recombination. Journal of virology 2004;78,1388013890 .

Basu J, Compitello G, Stromberg G, et al. Efficient assembly of de novo human artificial chromosomes from large genomic loci. BMC Biotechnol 2005;5:21.

Baumann BC, Stussi G, Huggel K, et al. Reactivity of human natural antibodies to endothelial cells from Galalpha(1,3)Gal-deficient pigs. Transplantation 2007;83,193-201.

Bhang SH, Kim JH, Yang HS, et al. Combined gene therapy with hypoxiainducible factor-1 alpha and heme oxygenase-1 for therapeutic angiogenesis. Tissue Eng Part A 2011;17:915-926.

Bieringer M. Cross-Species FISH Analysen von künstlichen Chromosomen in humanen und porcinen Zelllinien. Bachelor thesis chair of Livestock Biotechnology, 2008.

Bibikova M, Golic M, Golic KG, et al. Targeted chromosomal cleavage and mutagenesis in Drosophila using zinc-finger nucleases. Genetics 2002;161:11691175.

Bodnar AG, Quellette M, Frolkis M, et al. Extension of life-span by introduction of telomerase into normal human cells. Science 1998;279:349-352.

Bongoni AK, Kiermeier D, Schinder J, et al. Transgenic expression of human CD46 on porcine endothelium: Effect on coagulation and fibrinolytic cascades during ex vivo human-to-pig limb xenoperfusions. Transplantation 2015;99:2061-2069.

Bradley A, Evans M, Kaufmann MH, et al. Formation of germ-line chimaeras from embryo-derived teratocarcinoma cell lines. Nature 1984;309:255-256.

Brandl U, Michel S, Erhardt M, et al. Transgenic animals in experimental xenotransplantation models: orthotopic heart transplantation in the pig-tobaboon heart transplantation model. Transplant Proc 2007;39:577-578.

Brodbeck W, Liu J, Sperry C, et al. Localization of classical and alternative pathway regulatory activity within the decay-accelerating factor. J. Immunol. 1996;156:2528.

Bubeck D, Roversi P, Donev R, et al. Structure of human complement C8, a precursor to membrane attack. J Mol Biol. 2011;14;405(2):325-30.

Buser R, Montesano R, Garcia I, et al. Bovine microvascular endothelial cells immortalized with human telomerase. J Cell Biochem 2006;98:267-286. 
Byrne GW, Davies WR, Oi K, et al. Increased immunosuppression, not anticoagulation, extends cardiac xenograft survival. Transplantation 2006;82:1787-1791.

Byrne GW, McCurry KR, Martin MJ, et al. Transgenic pigs expressing human CD59 and decay-accelerating factor produce an intrinsic barrier to complementmediated damage. Transplantation 1997:15;63(1):149-155.

Cai M, Huttinger ZM, He H, et al. Transgenic over expression of ectonucleotide triphosphate diphosphohydrolase-1 protects against murine myocardial ischemic injury. J Mol Cell Cardiol. 2011;51:927-935.

Campbell W, Lazoura E, Okada N, et al. Inactivation of C3a and C5a octapeptides by carboxypeptidase $\mathrm{R}$ and carboxypeptidase $\mathrm{N}$. Microbiol Immunol 2002;46:131-134.

Capasso M, Durrant LG, Stacey M, et al. Costimulation via CD55 on Human CD4+T Cells Mediated by CD97. J Immunol 2006;177(2):1070-1077.

Caras IW, Weddell GN. Signal peptide for protein secretion directing glycophospholipid membrane anchor attachment. Science 1989;243:1196-1198

Carbery ID, Ji D, Harrington A, et al. Targeted Genome Modification in Mice Using Zinc Finger Nucleases. Genetics 2010;186:451-459.

Carlson DF, Wenfang T, Lillico SG, et al. Efficient TALEN-mediated gene knockout in livestock. PNAS 2012 109;43:17382-17387.

Cary N, Moody J, Yannoutsos N, et al. Tissue expression of human decay accelerating factor, a regulator of complement activation expressed in mice: a potential approach to complement activation expressed in mice: a potential approach to inhibition of hyperacute xenograft rejection. Transplant Proc. 1993;25:400-401.

Cascio SM. Novel strategies for immortalization of human hepatocytes. Artif Organs 2001;25:529-538.

Cavaliere FM, Scoarughi GL, Cimmion C. Interspecific transfer of mammalian artificial chromosomes between farm animals. Chromosome research 2009;17:507-517.

Chen RH, Naficy S, Logan JS, et al. Hearts from transgenic pigs constructed with CD59/DAF genomic clones demonstrate improved survival in primates. Xenotransplantation 1999;6,194-200.

Chiu CP, Harley CB. Replicative senescence and cell immortality: The role of telomeres and telomerase. Proc Soc Exp Biol Med 1997;214:99-106. 
Choo KH, Vissel B, Nagy A, et al. A survey of the genomic distribution of alpha satellite DNA on all the human chromosomes, and derivation of a new consensus sequence. Nuc. Acids Res. 1991;19(6):1179-1182.

Cermak T, Doyle EL, Christian M, et al. Efficient design and assembly of custom TALEN and other TAL effector-based constructs for DNA targeting. Nucleic Acids Res 2011;39:e82.

Cocuzzi ET, Bardenstein DS, Stavitsky A, et al. Upregulation of DAF (CD55) on orbital fibroblasts by cytokines: differential effects of TNF- $\beta$ and TNF- $\alpha$. Curr. Eye Res. 2001;23:86-92.

Colgan SP, Eltzschig HK, Eckle T, et al. Physiological roles for ecto-5'nucleotidase (CD73). Purinergic Signal. 2006;2:351-360.

Conway E, Van de W, Pollefeyt S, et al. The lectin-like domain of thrombomodulin confers protection from neutrophil-mediated tissue damage by suppressing adhesion molecule expression via nuclear factor kappaB and mitogen-activated protein kinase pathways. J Exp Med 2002;196:656-577.

Cooper DK, Koren E, Oriol R. Oligosaccharides and discordant xenotransplantation. Immunol Rev 1994;141:31-58.

Coornaert B, Baens M, Heyninck $\mathrm{K}$, et al. $\mathrm{T}$ cell antigen receptor stimulation induces MALT1 paracaspase-mediated cleavage of the NF- $\kappa$ B inhibitor A20. Nat. Immunol. Volume 2008;9(3):263-271.

Coren JS, Sternberg N. Construction of a PAC vector system for the propagation of genomic DNA in bacterial and mammalian cells and subsequent generation of nested deletions in individual library members. Gene 2001;264(1):11-18.

Cowan PJ, Chen CG, Shinkel TA, et al. Knock out of alpha1,3galactosyltransferase or expression of alpha1,2-fucosyltransferase further protects CD55- and CD59-expressing mouse hearts in an ex vivo model of xenograft rejection. Transplantation 1998;65(12):1599-1604.

Cowan PJ, Shinkel TA, Aminian A, et al. High-level coexpression of complement regulators on vascular endothelium in transgenic mice: CD55 and CD59 provide greater protection from human complement-mediated injury than CD59 alone. Xenotransplantation 1998;5:184.

Coyne KE, Hall SE, Thompson S, et al. Mapping of epitopes, glycosylation sites, and complement regulatory domains in human decay accelerating factor. J. Immunol. 1992;149:2906-2913.

Cozzi E, Tallacchini $\mathbf{M}$, Flanagan EB, et al. The International Xenotransplantation Association consensus statement on conditions for undertaking clinical trials of porcine islet products in type 1 diabetes - chapter 1 : 
Key ethical requirements and progress toward the definition of an international regulatory framework. Xenotransplantation 2009;16,203-214.

Cozzi E, Tucker AW, Langford GA, et al. Characterization of transgenic pigs for human decay-accelerating factor. Transplantation 1997;64:1383-1392.

Cozzi E, White DJ. The generation of transgenic pigs as potential organ donors for humans. Nat Med 1995;1:964.

Cui W, Zhao Y, Shan C, et al.: HBXIP upregulates CD46, CD55 and CD59 through ERK1/2/NF- $\mathrm{BB}$ signaling to protect breast cancer cells from complement attack. FEBS letters 2012;586:766-771.

Cui X, Ji D, Fisher DA, et al. Targeted interaction in rat and mouse embryos with zinc-finger nucleases. Nat. Biotechnol. 2011;29,64-67.

Dai Y, Vaught TD, Boone J, et al. Targeted disruption of the alpha1,3galactosyltransferase gene in cloned pigs. Nat Biotechnol. 2002;20,251-255.

Davies A, Simmons DL, Hale G, et al. CD59, an LY-6-like protein expressed in human lymphoid cells, regulates the action of the complement membrane attack complex on homologous cells. J. Exp. Med. 1989;170:637-654.

Denner J. Is porcine endogenous retrovirus (PERV) transmission still relevant? Transplant Proc. 2008;40(2):587-589.

Denning C, Burl S, Ainslie A, et al. Deletion of the alpha(1,3)galactosyl transferase /GGTA1 gene and the prion protein (PrP) gene in sheep. Nat Biotechnologie 2001;19:559-562.

Denning C, Dickinson P, Burl S, et al. Gene targeting in primary fetal fibroblasts from sheep and pig. Cloning Stem Cells 2001;3:221-231.

Deppenmeier S, Bock 0, Mengel M, et al. Health status of transgenic pigs expressing the human complement regulatory protein CD59. Xenotransplantation 2006;13:345-356.

Dieken ES, Epner EM, Fiering S, et al. Efficient modification of human chromosomal alleles using recombination-proficient chicken/human microcell hybrids. Nat Genet. 1996;12:174-182.

Dieckhoff B, Petersen B, Kues WA, et al. Knockdown of prcine endogenous retrovirus (PERV) expression by PERV-specific shRNA in transgenic pigs. Xenotransplantation 2008;15:36-45.

Dixit V, Mak TW. NF-kappaB signaling. Many roads lead to Madrid. Cell 2002;111,615-619. 
Dooldeniya MD, Warrens AN. Xenotransplantation: where are we today? J.R.Soc.Med.2003;96:111-117.

Dorling A, Lombardi G, Binns R, et al. Detection of primary direct and indirect human anti-porcine $\mathrm{T}$ cell responses using a porcine dendritic cell population. Eur J Immunol 1996;26:1378-1387.

Düwel M, Welteke V, Oeckinghaus A, et al. A20 Negatively Regulates T Cell

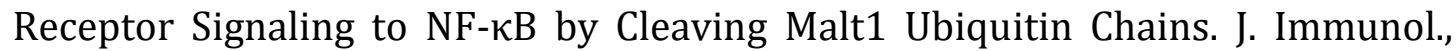
2009,182:7718-7728.

Ebersole TA, Ross A, Clark E, et al. Mammalian artificial chromosome formation from circular alphoid input DNA does not require telomere repeats. Hum. Mol. Genet. 2000;9:1623-1631.

Ege T, Ringertz NR. Preparation of microcells by enucleation of micronucleate cells. Exp. Cell Res. 1974;87:378-382.

Eisenstein RS, Garcia-Mayol D, Pettingell W, et al. Regulation of ferritin and heme oxygenase synthesis in rat fibroblasts by different forms of iron. Proc Natl Acad Sci USA 1991; 88:688-692.

Elliott B, Jasin M. Repair of double-strand breaks by homologous recombination in mismatch repair-defective mammalian cells. Mol Cell Biol. 2001;21:26712682.

Esmon CT. The regulation of natural anticoagulant pathways. Science 1987;235:1348-1352.

Evans MJ and Kaufmann MH. Establishment in culture of pluripotential cells from mouse embryos. Nature 1981;292:154-156.

Ewulonu UK, Ravi L, Medof ME. Characterization of the decay-accelerating factor gene promoter region. Proc. Nati. Acad. Sci. USA 1991;88:4675-4679.

Ezzelarab M, Ezzelarab C, Wilhite $T$, et al. Genetically-modified pig mesenchymal stromal cells: xenoantigenicity and effect on human T-cell xenoresponses. Xenotransplantation 2011;18:183-195.

Ferris CD, Jaffrey SR, Sawa A, et al. Haem oxygenase-1 prevents cell death by regulating cellular iron. Nat Cell Biol 1999;1:152-157.

Fisicaro N, Londrigan SL. Brady JL, et al. Versatile co-expression of graftprotective proteins using 2A-linked cassettes. Xenotransplantation 2011;18:121130.

Flisikowska T, Thorey IS, Offner S, et al. Efficient immunoglobin gene disruption and targeted replacement in rabbit using zinc finger nucleases. PLoS ONE 2011;6:e21045. 
Forte P, Lilienfeld B, Baumann B, et al. Human NK cytotoxicity against porcine cells is triggered by NKp44 and NKG2D. J. Immunol. 2005;175:5463-5470.

Fournier RE, Ruddle FH. Microcell-mediated transfer of murine chromosomes into mouse, Chinese hamster, and human somatic cells. Proc. Nat. Acad. Sci. USA 1977;74:319-323.

Frengen E, Weichenhan D, Zhao B, et al. A modular, positive selection bacterial artificial chromosome vector with multiple cloning sites. Genomics 1999;58(3):250-253.

Friedrich G, Soriano P. Promoter traps in embryonic stem cells: a genetic screen to identify and mutate developmental genes in mice. Genes Dev 1991;5:1513-1523.

Fulcher CA, Gardiner JE, Griffin JH, et al. Proteolytic inactivation of human factor VIII procoagulant protein by activated human protein $\mathrm{C}$ and its analogy with factor V. Blood 1984;63:486-489.

Galili U. Interaction of the natural anti-Gal antibody with $\alpha$-galactosyl epitopes: a major obstacle for xenotransplantation in humans. Immunol. Today $1993 ; 14,480-482$.

Galili U, Macher BA, Buehler J, et al. Human natural anti-alpha-galactosyl IgG II. The specific recognition of alpha (1-3)-linked galactose residues. J Exp Med 1985:162:573-582.

Garkavenko 0, Wynyard S, Nathu D, et al. The first clinical xenotransplantation trial in New Zealand: Efficacy and safety. Xenotransplantation 2012:19:2-22.

Good AH, Cooper DK, Malcolm AJ, et al. Identification of carbohydrate structures that bind human antiporcine antibodies: implications for discordant xenografting in humans. Transplant Proc 1992;24:559-562.

Gordon EJ, Woda BA, Shultz LD, et al. Rat xenograft survival in mice treated with donor-specific transfusion and anti-CD154 antibody is enhanced by elimination of host CD4+ cells. Transplantation 2001;71:319-327.

Griffiths AJF. Transcription: an overview of gene regulation in eukaryotes. An Introduction to Genetic Analysis 2000, 7th edition.

Groux H, Huet S, Aubrit F, et al. A 19-kDa human erythrocyte molecule H19 is involved in rosettes, present on nucleated cells, and required for T cell activation. Comparison of the roles of $\mathrm{H} 19$ and LFA-3 molecules in T cell activation. J. Immunol. 1989;142:3013-3020. 
Guiducci C, Ascenzioni F, Auriche C, et al. Use of a human minichromosome as a cloning and expression vector for mammalian cells. Hum Mol Genet 1999;8:1417-1424.

Hackett JA, Feldser DM, Greider CW, et al. Telomere dysfunction increases mutation rate and genomic instability. Cell 2001;106:275-286.

Hadam M. Leucocyte Typing IV: Handbook of Experimental Immunology. Oxford Univ. Press 1989:720-722.

Hammer R, Pursel V, Rexroad C, et al. Production of transgenic rabbits, sheep and pigs by microinjection. Nature 1985; 315: 680-683.

Hanish JP, Yanowitz JL, de Lange T, et al. Stringent sequence requirements for the formation of human telomeres. Proc. Nat. Acad. Sci. USA 1994;91:8861-8865.

Hara H, Long C, Lin YJ, et al. In vitro investigation of pig cells for resistance to human antibody-mediated rejection. Transpl Int. 2008;21:1163- 1174.

Harrington J, Van Bokkelen G, Mays R, et al. Formation of de novo centromeres and construction of first-generation human artificial microchromosomes. Nature Genetics 1997;15(4):345-355.

Hasty P, Bradley A. Gene targeting vectors for mammalian cells, in: Joyner A.L. (Ed.), Gene targeting: A Practical Approach, IRL Press, Oxford 1993:1-31.

Hasty P, Rivera-Perez J, Bradley A. The length of homology required for gene targeting in embryonic stem cells. Mol. Cell. Biol. 1991;11:5586-5591.

Hauschild J, Petersen B, Santiago Y, et al. Efficient generation of a biallelic knockout in pigs using zinc-finger nucleases. PNAS USA 2011;108,12013-12017.

Hernandez D, Mee PJ, Martin JE, et al. Transchromosomal mouse embryonic stem cell lines and chimeric mice that contain freely segregating segments of human chromosome 21. Hum Mol Genet 1999;8:923-933.

Higashi H, Naiki M, Matuo S, et al. Antigen of "serum sickness" type of heterophile antibodies in human sera: identification as gangliosides with $\mathrm{N}$ glycolylneuraminic acid. Biochem Biophys Res Commun 1977;79:388-395.

Holla VR, Wang D, Brown JR, et al. Prostaglandin E2 regulates the complement inhibitor CD55/decay-accelerating factor in colorectal cancer. J. Biol. Chem. 2005;280:476-483.

Hombach-Klonisch S, Pocar P, Kauffold J, et al. Dioxin exerts anti-estrogenic actions in a novel dioxin-responsive telomerase-immortalized epithelial cell line of the porcine oviduct. Toxicol Sci 2006;90:519-528. 
Hosokawa M, Nonaka M, Okada N, et al. Molecular cloning of guinea pig membrane cofactor protein: preferential expression in testis. J. Immunol. 1996;157:4946-4952.

Ide $\mathbf{K}$, Wang $\mathbf{H}$, Tahara $\mathbf{H}$, et al. Role for CD47-SIRP $\alpha$ signaling in xenograft rejection by macrophages. Proc. Natl. Acad. Sci. USA 2007;104:5062-5066.

Ikeno $\mathbf{M}$, Inagaki $\mathbf{H}$, Nagata $\mathbf{K}$, et al. Generation of human artificial chromosomes expressing naturally controlled guanosine triphosphate cyclohydrolase I gene. Genes to Cell 2002;7:1021-1032.

Ikeno M, Suzuki N, Hasegawa $\mathbf{Y}$, et al. Manipulating transgenes using a chromosome vector. Nuc Acids Res 2009;37(6):e44.

Irion S, Luche H, Gadue P, et al. Identification and targeting of the ROSA26 locus in human embryonic stem cells. Nat Biotechnol. 2007;25:1477-1482.

Ito M, Maruyama T, Saito $\mathbf{N}$, et al. Killer cell lectin-like receptor G1 binds three members of the classical cadherin family to inhibit NK cell cytotoxicity. J. Exp. Med. 2006;203:289-295.

Janzen MA, Buoen LB, Zhao F, et al. Characterization of a swine chromosomespecific centromeric higher-order repeat. Mammalian Genome 1998;10:579-584.

Jeong YH, Park CH, Jang GH, et al. Production of multiple transgenic Yucatan miniature pigs expressing human complement regulatory factors, human CD55, CD59 and H-transferase genes. PLoS ONE 2013;8(5):e63241.

Jiang W, Zhou H, Bi H, et al. Demonstration of CRISPR/Cas9/sgRNA-mediated targeted gene modification in Arabidopsis, tobacco, sorghum and rice. Nucl. Acids Res. 2013:10-31.

Jin D, Lee S, Choi J, et al. Targeting efficiency of alpha-1,3-galactosyltransferase gene in pig fetal fibroblast cells. Exp.Mol. Med. 2003;35:572-577.

Jin R, Greenwald A, Peterson M, et al. Human monocytes recognize porcine endothelium via the interaction of galectin 3 and $\alpha \mathrm{Gal}$. J. Immunol. 2006;177:1289-1295.

Jinek M, Chylinski K, Fonfara I, et al. A Programmable Dual-RNA-Guided DNA Endonuclease in Adaptive Bacterial Immunity. Science 2013;337:816-821.

Johnstone RW, Loveland BE, McKenzie IF. Identification and quantification of complement regulator CD46 on normal human tissues. Immunology 1993;79:341.

Kaczmarek E, Koziak K, Sevigny J, et al. Identification and characterization of CD39/vascular ATP diphosphohydrolase. J Biol Chem 1996;271:33116-33122. 
Kakeda M, Hiratsuka M, Nagata $\mathbf{K}$, et al. Human artificial chromosome (HAC) vector provides long-term therapeutic transgene expression in normal human primary fibroblasts. Gene Ther. 2005;12(10):852-856.

Kakeda M, Nagata K, Osawa K, et al. A new chromosome 14-based human artificial chromosome (HAC) vector system for efficient transgene expression in human primary cells. Biochemical and Biophysical Research Com. 2011;415(3):439-444.

Karin M, Ben-Neriah Y. Phosphorylation meets ubiquitination: the control of NF- $\kappa B$ activity. Annu. Rev. Immunol. 2000;18:621-663.

Karnauchow TM, Dawe S, Lublin DM, et al. Short consensus repeat domain 1 of decay-accelerating factor is required for enterovirus 70 binding. J. Virol. 1998;72:9380-9383.

Katoh M, Kazuki Y, Kazuki K, et al. Exploitation of the interaction of measles virus fusogenic envelope proteins with the surface receptor CD46 on human cells for microcell-mediated chromosome transfer. BMC Biotechnology 2010;10:37.

Katori M, Busuttil RW, Kupiec-Weglinski JW, et al. Heme oxygenase-1 system in organ transplantation. Transplantation 2002;74:905-912.

Kazuki Y, Hoshiya $\mathbf{H}$, Takiguchi M, et al. Refined human artificial chromosome vectors for gene therapy and animal transgenesis. Gene therapy 2011;18(4):384393.

Kim YG, Cha J, Chandrasegaran S. Hybrid restriction enzymes: Zinc finger fusions to FokI cleavage domain. Proc Natl Acad Sci USA 1996;93:1156-1160.

Klymiuk N, Buerck van L, Bähr A, et al. Xenografted Islet Cell Clusters from INSLEA29Y Transgenic Pigs Rescue Diabetes and Prevent Immune Rejection in Humanized Mice. Diabetes 2012;61:1527-1531.

Kobayashi T, Kato-Itoh M, Yamaguchi T, et al. Identification of rat ROSA26 locus enables generation of knock-in rat lines ubiquitously expressing tdTomato. Stem Cells Dev 2012; 21:2981-2986.

Kolber-Simonds D, Lai L, Watt SR, et al. Production of alpha-1,3galactosyltransferase null pigs by means of nuclear transfer with fibroblasts bearing loss of heterozygosity mutations. Proc Natl Acad Sci USA 2004;101:7335-7340.

Koziak K, Sevigny J, Robson SC, et al. Analysis of CD39/ATP diphosphohydrolase (ATPDase) expression in endothelial cells, platelets and leukocytes. Thromb Haemost 1999;82:1538-1544.

Kraner S, Christan S, Zoller M et al. Reconstructing functional gene loci using very long PCR. ESHG, Nice, France 2007;16-19-06. 
Krikos A, Laherty CD, Dixit VM. Transcriptional activation of the tumor necrosis factor $\alpha$-inducible zinc finger protein, A20, is mediated by $\kappa B$ elements. J.Biol. Chem. 1992;267:17971-17976.

Kuroiwa Y, Kasinathan P, Choi YJ, et al. Cloned transchromosomic calves producing human immunoglobulin. Nat Biotechnol 2002;20:889-894.

Kuroiwa Y, Kasinathan P, Matsushita $\mathbf{H}$, et al. Sequential targeting of the genes encoding immunoglobulin-mu and prion protein in cattle. Nat. Genet. 2004;36:775-780.

Kuroiwa Y, Shinohara T, Notsu T, et al. Efficient modification of a human chromosome by telomere-directed truncation in high homologous recombination-proficient chicken DT40 cells. Nucleic Acids Res. 1998;26:34473448.

Kuwaki K, Tseng YL, Dor FJ, et al. Heart transplantation in baboons using alpha 1,3-galactosyltransferase gene knockout pigs as donors: initial experience. Nat Med 2005;11:29-31.

Lai L, Kolber-Simonds D, Park KW, et al. Production of alpha-1,3galactosyltransferase knockout pigs by nuclear transfer cloning. Science 2002;295,1089-1092.

Laner A, Schwarz T, Christan S, et al. Suitability of a CMV/EGFP cassette to monitor stable expression from human artificial chromosomes but not transient transfer in the cells forming viable clones. Cytogenet. Genome Res. 2004;107(12):9-13.

Laner A, 2005: Entwicklung von DNA- und Expressions-Markern zum Nachweis von menschlichen künstlichen Chromosomen und in vivo Transfektionsanalyse verbesserter HAC Vektoren, Dissertation 2005, LMU München.

Lee EG, Boone DL, Chai S, et al. Failure to regulate TNF-induced NF-kappaB and cell death responses in A20-deficient mice. Science 2000;289,2350-2354.

Lee HW, Blasco A, Gottlieb GJ, et al. Essential role of mouse telomerase in highly proliferative organs. Nature 1998;392:569-574.

Lenschow DJ, Zeng Y, Thistlethwaite JR, et al. Long-term survival of xenogeneic pancreatic islet grafts induced by CTLA4Ig. Science 1992;257:789792.

Li P, Burlak C, Estrada J, et al. Identification and cloning of the porcine ROSA26 promoter and its role in transgenesis. Transplantation Technology 2014; 10.7243/2053-6623-2-1. 
Li S, Flisikowsa T, Kurome M, et al. Dual Fluorescent Reporter Pig for Cre Recombination: Transgene Placement at the ROSA26 Locus. PLoSONE 2014;9(7): e102455.

Lilienfeld B, Garcia-Borges C, Crew M, et al. Porcine UL16-binding protein 1 expressed on the surface of endothelial cells triggers human NK cytotoxicity through NKG2D. J. Immunol. 2006;177:2146-2152.

Lin SS, Hanaway MJ, Gonzalez-Stawinski GV, et al. The role of anti-Galalpha1$3 \mathrm{Gal}$ antibodies in acute vascular rejection and accommodation of xenografts. Transplantation 2000;70:1667-1674.

Lindahl G, Sjöbring U, Johnsson E. Human complement regulators: a major target for pathogenic microorganisms. Curr. Opin. Immunol. 2000;12:44-51.

Linsley P, Brady W, Urnes M, et al. CTLA4 is a second receptor for the B cell activation antigen B7. J Exp Med 1991;174:561-569.

Linsley P, Greene J, Brady W, et al. Human B7-1 (CD80) and B7-2 (CD86) bind with similar avidities but distinct kinetics to CD28 and CTLA4 receptors. Immunity 1994;1:793-801.

Liszewski MK, Atkinson JP. Membrane cofactor protein (MCP; CD46) isoforms differ in protection against the classical pathway of complement. J Immunol. 1996;156:4415-4421.

Liszewski MK, Post TW, Atkinson JP. Membrane cofactor protein (MCP or CD46): newest member of the complement activation gene cluster. Annu Rev Immunol 1991;9:431-455.

Liu J, Miwa T, Hilliard B, et al. The complement inhibitory protein DAF (CD55) suppresses T cell immunity in vivo. J.Exp. Med. 2005;201:567-577.

Liu P, Jenkins NA, Copeland NG. A highly efficient recombineering-based method for generating conditional knockout mutations. Genome Res. 2003;13(3):476-484.

Louis N, Hamilton K, Kong T, et al: HIF-dependent induction of apical CD55 coordinates epithelial clearance of neutrophils. FASEB J. 2005;19:950-959.

Loveland BE, Milland J, Kyriakou P, et al. Characterization of a CD46 transgenic pig and protection of transgenic kidneys against hyperacute rejection in non-immunosuppressed baboons. Xenotransplantation 2004;11:171-183.

Lutz AJ, Li P, Estrada JL, et al. Double knockout pigs deficient in Nglycolylneuraminic acid and Galactose $\alpha-1,3-$ Galactose reduce the humoral barrier to xenotransplantation. Xenotransplantation 2013;20:27-35. 
Mali P, Kevin ME, George MC. Cas 9 as a versatile tool for engineering biology. Nature methods 2013;10.10:957-963.

Marusawa H, Matsuzawa S, Welsh K, et al. HBXIP functions as a cofactor of surivin in apoptosis suppression. EMBO J. 2003;22(11):2729-2740.

Mason JC, Yarwood H, Sugars K, et al. Induction of decay-accelerating factor by cytokines or the membrane-attack complex protects vascular endothelial cells against complement deposition. Blood 1999;94:1673-1682.

Mason JC, Lidington EA, Ahamad SR, et al. bFGF and VEGF synergistically enhance endothelial cytoprotection via decay-accelerating factor induction. Am. J. Physiol. 2002;282:C578-C587.

Mason JC, Steinberg R, Lidington EA, et al., 2004: Decay-accelerating factor induction on vascular endothelium by vascular endothelial growth factor (VEGF) is mediated via a VEGF receptor-2 (VEGF-R2)- and protein kinase C- $\alpha / \varepsilon$ $(\mathrm{PKC} \alpha / \varepsilon)$-dependent cytoprotective signaling pathway and is inhibited by cyclosporine. J. Biol. Chem. 2004;279:41611-41618.

McCreath K, Howcroft J, Campbell K, et al. Production of gene-targeted sheep by nuclear transfer from cultured somatic cells. Nature 2000;405:1066-1069.

McCurry KR, Kooyman DL, Diamond LE, et al. Transgenic expression of human complement regulator proteins in mice results in diminished complement deposition during organ xenoperfusion. Transplantation 1995;59:1177-1182.

McGrath J, Solter D. Inability of mouse blastomere nuclei transferred to enucleated zygotes to support development in vitro. Science 1984;226:13171319.

McGregor CG, Ricci D, Miyagi N, et al. Human CD55 expression blocks hyperacute rejection and restricts complement activation in Gal knockout cardiac xenografts. Transplantation 2012;93(7):686-692.

McGregor CG, Davies WR, Oi K, et al. Cardiac xenotransplantation: recent preclinical progress with 3-month median survival. J. Thorac. Cardiovasc. Surg. 2005;130:844-851.

McNearney T, Ballard L, Seya T, et al. Membrane cofactor protein of complement is present on human fibroblast, epithelial and endothelial cells. J Clin Invest 1989;84:538-545.

Meaburn KJ, Parris CN, Bridger JM. The manipulation of chromosomes by mankind: the uses of microcell-mediated chromosome transfer: Chromosoma 2005; 114:263-274. 
Medof ME, Kinoshita T, Nussenzweig V. Inhibition of complement activation on the surface of cells after incorporation of decay-accelerating factor (DAF) into their membranes. J. Exp. Med. 1984;160:1558-1578.

Medof ME, Walter EI, Rutgers JL, et al. Identification of the complement decayaccelerating factor (DAF) on epithelium and glandular cells and in body fluids. J.Exp. Med. 1987;165:848-864.

Meija JE, Alazami A, Willmott A, et al. Efficiency of de novo centromere formation in human artificial chromosomes. Genomics 2002;79:297-304.

Merrick JM, Zadarlik K, Milgrom F. Characterization of the HanganutziuDeicher (serum sickness) antigen as gangliosides containing Nglycolylneuraminic acid. Int Arch Allergy Appl Immunol 1978; 57:477-480.

Meyer M, Hrabé de Angelis M, Wurst W, et al. Gene targeting by homologous recombination in mouse zygotes mediated by zinc-finger nucleases. Proc. Natl. Acad. Sci. USA 2010;107:15022-15026.

Michler RE. Xenotransplantation: risks, clinical potential and future prospects. Emerg Infect Dis. 1996;2:64-70.

Milland J, Christiansen D, Thorley BR, et al. Translation is enhanced after silent nucleotide substitutions in A+T-rich sequences of the coding region of CD46 cDNA. Eur J Biochem. 1996;238(1):221-30.

Miller JC, Tan S, Qiao G, et al. A TALE nuclease architecture for efficient genome editing. Nat Biotechnol 2011;29:143-148.

Mir B, Piedrahita J. Nuclear localization signal and cell synchrony enhance gene targeting efficiency in primary fetal fibroblasts. Nucleic Acids Res. 2004;32:25.

MIT report by Regalado A. Surgeons smash records with pig-to-primate organ transplants. MIT technology review 2015.

Miwa T, Nonaka M, Okada N, et al. Molecular cloning of rat and mouse membrane cofactor protein (MCP, CD46): preferential expression in testis and close linkage between the mouse MCP and CR2 genes on distal chromosome 1. Immunogenetics 1998;48:363-371.

Miyagawa S, Fukuta D, Kitano E, et al. Effect of tandem forms of DAF(CD55) on complement-mediated xenogeneic cell lysis. Xenotransplantation 2006;13:433439.

Miyagawa S, Hirose $\mathbf{H}$, Shirakura $\mathbf{R}$, et al. The mechanism of discordant xenograft rejection. Transplantation 1988;46:825-830. 
Miyagawa S, Ikawa M, Kominami K, et al. The regulation of membrane cofactor protein (CD46) expression in transgenic mice: the omportance of the first $125 \mathrm{BP}$ of the 3'untranslated region. Translant Proc 1997;29:941-942.

Miyagawa S, Kubo T, Matsunami K, et al. Delta-Short Consensus Repeat 4Decay Accelerating Factor (DAF:CD55) Inhibits Complement-Mediate cytolysis bur Not NK Cell-Mediated Cytolysis. J. Immunol. 2004;173:3945-3952.

Miyagawa S, Yamamoto A, Matsunami K, et al. Complement regulation in the GalT KO era. Xenotransplantation 2010;17:11-25.

Mohiuddin MM, Singh AK, Corcoran PC, et al. Genetically engineered pigs and target-specific immunomodulation provide significant graft survival and hope for clinical cardiac xenotransplantation. J Thorac Cardiovasc Surg 2014;148(3):1106-1113.

Mollnes TE, Fiane AE. Perspectives on complement in xenotransplantation. Mol Immunol 2003;40:135.

Mora M, Mulder LCF, Lazzeri M, et al. Protection from complement-mediated injury in livers and kidneys of transgenic mice expressing human complement regulators. Xenotransplantation 1996;3:63-68.

Morozumi K, Kobayashi T, Usami T, et al. Significance of histochemical expression of Hanganutziu-Deicher antigens in pig, baboon and human tissues. Transplant Proc 1999;31:942-944.

Mueller NJ, Takeuchi Y, Mattiuzzo G, et al. Microbial safety in xenotransplantation. Current opinion in organ transplantation 2011;16:201-206.

Mueller So, Tahara H, Barrett JC, et al. Immortalization of mammary cells from estrogen receptor alpha knock-out and wild-type mice. In Vitro Cell Dev Biol Anim 2000;36: 620-624.

Mulder LC, Rossini M, Mora M. Human CD46 aberrant splicing in transgenic mice. Gene 1997;168: 83-86.

Murakami H, Takahagi Y, Yoshitatsu M, et al. Porcine MCP Promoter Directs High Level Expression of Human DAF (CD55) in Transgenic Mice. Immunobiology 2000;201(5):583-597.

Murakami H, Nagashima $\mathbf{H}$, Takahagi $\mathbf{Y}$, et al. Transgenic pigs expressing human decay-accelerating factor regulated by porcine MCP gene promoter. Mol Reprod Dev. 2002;61(3):302-311.

Murray A, Khodadoust M, Pober J, et al. Porcine aortic endothelial cells activate human T cells: direct presentation of MHC antigens and costimulation by ligands for human CD2 and CD28. Immunity 1994;1:57-63. 
Nguyen DH, Tangvoranuntakul P, Varki A. Effects of natural human antibodies against a nonhuman sialic acid that metabolically incorporates into activated and malignant immune cells. J Immunol 2005;175:228-236.

Niemann H, Verhoeyen E, Wonigeit $\mathrm{K}$, et al. Cytomegalovirus early promoter induced expression of hCD59 in porcine organs provides protection against hyperacute rejection. Transplantation 2001;72:1898-1906.

Nonaka M, Takenaka $\mathbf{0}$, Okada $\mathbf{N}$, et al. A new repetitive sequence uniquely present in the decay-accelerating factor genes. Immunogenetics 1998;47:246255.

Nomura M, Tsujimura A, Shida $\mathbf{K}$, et al. Membrane and secretory forms of mouse membrane cofactor protein (CD46) generated from a single gene through alternative splicing. Immunogenetics 1999;50:245-254.

Nowak-Imialek M, Kues WA, Rudolph C, et al. Preferential Loss of Porcine Chromosomes in Reprogrammed Interspecies Cell Hybrids. Cellular Reprogramming 2010;12:55-65.

Oldham E, Velardo M, Platt J, et al. High-level tissue specific expression of human CD59, MCP and DAF proteins from genomic clones in transgenic mice. Transplant Proc 1996;28:693.

Oh HY, Jin X, Kim GG, et al. Characteristics of primary and immortalized fibroblast cells derived from the miniature ad domestic pigs. BMC Cell Biology 2007;8:20.

Opipari AW, Boguski MS, Dixit VM. The A20 cDNA induced tumor necrosis fator alpha encodes a novel type of zinc finger protein. J. Biol. Chem. 1990;265:14705-14708.

Oriol R, Ye Y, Koren E, et al. Carbohydrate antigens of pig tissues reacting with human natural antibodies as potential targets for hyperacute vascular rejection in pig-to-man organ xenotransplantation. Transplantation 1993;56:1433-1442.

Oropeza M, Petersen B, Carnwath JW, et al. Transgenic expression of the human A20 gene in cloned pigs provides protection against apoptotic and inflammatory stimuli. Xenotransplantation 2009;16:522-534.

Oshimura M, Katoh M. Transfer of human artificial chromosome vectors into stem cells. Reprod Biomed Online 2008;16:57-69.

Osuka F, Endo Y, Higuchi M, et al. Molecular cloning and characterization of novel splicing variants of human decay-acceleration factor. Genomics 2006;88:316-322. 
Pan X, Du W, He S, et al. Immortalization of porcine hepatocyte lines by transfection with simian virus 40 large $\mathrm{T}$ antigen and human telomerase reverse transcriptase. Ch J Infec Dis 2008;26:406-409.

Paulis M, Bensi M, Orioli D, et al. Transfer of a human chromosomal vector from a hamster cell line to a mouse embryonic stem cell line. Stem Cells 2007;25:2543-2550.

Petersen B, Ramackers W, Lucas-Hahn A, et al. Transgenic expression of human heme oxygenase-1 in pigs confers resistance against xenograft rejection during ex vivo perfusion of porcine kidneys. Xenotransplantation 2011;18:355368.

Petersen B, Ramackers W, Tiede A, et al. Pigs transgenic for human thrombomodulin have elevated production of activated protein $\mathrm{C}$. Xenotransplantation 2009;16:486-495.

Phelps C, Ball S, Vaught T, et al. Production and characterization of transgenic pigs expressing porcine CTLA4-Ig. Xenotransplantation 2009;16:477-485.

Phelps CJ, Koike C, Vaught TD, et al. Production of alpha 1,3galactosyltransferase-deficient pigs. Science 2003;299:411-414.

Philbrick WM, Palfree RG, Maher SE, et al. The CD59 antigen is a structural homologue of murine Ly- 6 antigens but lacks interferon inducibility. Eur. J. Immunol. 1990;20,87-92.

Pierson RN. Antibody-mediated xenograft injury: mechanisms and protective strategies. Transplant Immunology 2009;21,65-69.

Platt JL. New directions for organ transplantation. Nature 1998;392:11-17.

Platt JL, Fischel RJ, Matas AJ, et al. Immunopathology of hyperacute xenograft rejection in a swine-to-primate model. Transplantation 1991;52:214-220.

Poggiali P, Scoarughi GL, Lavitrano M, et al. Construction of a swine artificial chromosome: a novel vector for transgenesis in the pig. Biochimie 2002;84:1143-1150.

Pollard AJ, Flanagan BF, Newton DJ, et al. A novel isoforms of human membrane cofactor protein (CD46) mRNA generated by intron retention. Gene 1998;212:39-47.

Porteus MH, Carroll D. Gene targeting using zinc finger nucleases. Nat Biotechnol 2005;23:967-973.

Post TW, Arce MA, Liszewski MK, et al. Structure of the gene for human complement protein decay accelerating factor. J. Immunol. 1990;144:740-744. 
Post TW, Liszewski MK, Adams EM, et al. Membrane cofactor protein of the complement system: alternative splicing of serine/threonine/proline-rich exons and cytoplasmic tails produces multiple isoforms that correlate with protein phenotype. J Exp Med 1991;174:93-102.

Powell RM, Ward T, Goodfellow I, et al. Mapping the binding domains on decay accelerating factor (DAF) for haemagglutinating enteroviruses: implications for the evolution of a DAF-binding phenotype. J. Gen. Virol 1999;80:3145-3152.

Purcell DF, Russell SM, Deacon NJ, et al. Alternatively spliced RNAs encode several isoforms of CD46 (MCP), a regulator of complement activation. Immunogenetics 1991;33:335-344.

Reyon D, Tsai SQ, Khayter C, et al. FLASH assembly of TALENs for highthroughput genome editig. Nat Biotechnol 2012,30:460-465.

Ricklin D, Lambris JD. Complement-targeted therapeutics. Nat Biotechnol 2007;25:1265-1275.

Rogers C, Hao Y, Rokhlina T, et al. Production of CFTR-null and CFTRDeltaF508 heterozygous pigs by adeno-associated virus-mediated gene targeting and somatic cell nuclear transfer. J. Clin. Invest 2008;118:1571-1577.

Russell D, Hirata R. Human gene targeting by viral vectors. Nat. Genet. 1998;18:325-330.

Saethre M, Baumann BC, Fung M, et al. Characterization of natural human antinon-gal antibodies and their effect on activation of porcine gal-deficient endothelial cells. Transplantation 2007;84:244-250.

Saffery R, Choo KH. Strategies for engineering human chromosomes with therapeutic potential. J Gene Med 2002;4:5-13.

Sakata Y, Curriden S, Lawrence D, et al. Activated protein C stimulates the fibrinolytic activity of cultured endothelial cells and decreases antiactivator activity. Proc Nat Acad Sci USA 1985;82:1121-1125.

Sandrin MS, Fodor WL, Mouhtouris E, et al. Enzymatic remodelling of the carbohydrate surface of a xenogenic cell substantially reduces human antibody binding and complement-mediated cytolysis. Nat Med 1995;1:1261-1267.

Sato K, Balla J, Otterbein L, et al. Carbon monoxide generated by heme oxygenase-1 suppresses the rejection of mouse-to-rat cardiac transplants. Journal of immunology 2001;166:4185-4194.

Sawada R, Ohashi K, Anaguchi H, et al. Isolation and expression of the fulllength cDNA encoding CD59 antigen of human lymphocytes.DNA Cell Biol. 1990; 9,213-220. 
Schäffauer AJ. Klonierung und Charakterisierung einer genomischen Schweinebank mit zentromerischen Sequenzen im PAC-Vektor pTT. Master Thesis chair of Livestock Biotechnology 2008

Sedivy J, Dutriaux A. Gene targeting and somatic cell genetics - A rebirth or a coming of age? Trends Genet. 1999;15:88-90.

Seebach JD, Comrack C, Germana S, et al. HLA-Cw3 expression on porcine endothelial cells protects against xenogeneic cytotoxicity mediated by a subset of human NK cells. J. Immunol 1997;159:3655-3661.

Sendai Y, Sawada T, Urakawa M, et al. Heterozygous disruption of the alpha1,3-galactosyltransferase gene in cattle. Transplantation 2003;76:900-902.

Sendai Y, Sawada T, Urakawa M, et al. Alpha 1,3-Galactosyltransferase-gene knockout in cattle using a single targeting vector with loxP sequences and creexpressing adenovirus. Transplantation 2006;81:760-766.

Seya T, Turner JR, Atkinson JP. Purification and characterization of a membrane protein (gp45-70) that is a cofactor for cleavage of C3b and C4b. J Exp Med 1986;163:837-855.

Shinohara T, Sakurada C, Suzuki T, et al. Pro-carboxypeptidase R cleaves bradykinin following activation. Int Arch Allergy Immunol 1994;103:400-404.

Shizuya H, Birren B, Kim UJ, et al. Cloning and stable maintenance of 300kilobase-pair fragments of human DNA in Escherichia coli using an F-factorbased vector. Proc. Natl. Acad. Sci. USA. 1992;89:8794-8797.

Shizuya H, Kouros-Mehr H. The development and applications of the bacterial artificial chromosome cloning system. Keio J Med 2001;50(1):26-30.

Siegel J, Grey S, Lesnikoski B, et al. Xenogenic endothelial cells activate human prothrombin. Transplantation 1997;64:888-896.

Stefanova I, Hilgert I, Kristofova $\mathbf{H}$, et al. Characterization of a broadly expressed human leucocyte surface antigen MEM-43 anchored in membrane through phosphatidylinositol. Mol. Immunol. 1989;26:153-161.

Stone N, Fan JB, Willour V, et al. Construction of a 750-kb Bacterial Clone Contig and Restricion Map in the Region of Human Chromosome 21 Containing the Progressive Myoclonus Epilepsy Gene. Gemone Research 1996;6:218-225.

Stubblefield E, Pershouse M. Direct Formation of microcells from Mitotic Cells for Use in Chromosome Transfer. Somatic Cell Mol Genetics 1992;18:485-491.

Sun X, Yan Z, Yi Y, et al. Adeno-associated virus-targeted disruption of the CFTR gene in cloned ferrets. J. Clin. Invest. 2008;118:1578-1583. 
Suzuki N, Nishii K, Okazaki T, et al. Human Artificial Chromosomes Constructed Using the Bottom-up Strategy Are Stably Maintained in Mitosis and Efficiently Transmissible to Progeny Mice. J Biol Chem 2006;281:26615-26623.

Suzuki K, Stenflo J, Dahlback B, et al. Inactivation of human coagulation factor V by activated protein C. J Biol Chem 1983;258:1914-1920.

Suzuki N, Nishii K, Okazaki T, et al. Human Artificial Chromosomes Constructed Using the Bottom-up Strategy Are Stably Maintained in Mitosis and Efficiently Transmissible to Progeny Mice. The Journal of Biological Chemistry 2006;281:26615-26623.

Tabata T, de Perrot $M$, Keshavjee S, et al. Accommodation after lung xenografting from hamster to rat. Transplantation 2003;75:607-612.

Taghian DG, Nickoloff JA. Chromosomal double-strand breaks induce gene conversion at high frequency in mammalian cells. Mol ell Biol. 1997;17:63866393.

Taniguchi S, Neethling FA, Korchagina EY, et al. In vivo immunoadsorption of antipig antibodies in baboons using specific Gal(alpha)1-3Gal column. Transplantation 1996;62:1379-1384.

Taylor F, Peer G, Lockhart M, et al. Endothelial cell protein C receptor plays an important role in protein C activation in vivo. Blood 2001;97:1685-1688.

Thomas K, Folger K, Capecchi M. High frequency targeting of genes to specific sites in the mammalian genome. Cell 1986;44:419-428.

Tieng V, Le Bouguenec C, du Merle L, et al. Binding of Escherichia coli adhesin AfaE to CD55 triggers cell-surface expression of the MHC class I-related molecule MICA. Proc. Natl. Acad. Sci. USA 2002;99:2977-2982.

Ting AT, Pimentel-Muinos FX, Seed B. RIP mediates tumor necrosis factor receptor 1 activation of NF-kappaB but not Fas/APO-1-initiated apoptosis. EMBO J. 1996;15:6189-6196.

Tomizuka K, Yoshida H, Uejima $\mathbf{H}$, et al. Functional expression and germline transmission of a human chromosome fragment in chimaeric mice. Nature Genetics 1997;16:133-143.

Tress ML, Martelli PL, Frankish A, et al. The implications of alternative splicing in the ENCODE protein complement. Proc Natl Acad Sci USA 2007; 104: 54955500 .

Tsujimura A, Nunoue K, Inoue N, et al. Three soluble form messages of murine CD46 are produced through alternative mRNA splicing. J. Biochem. 2001;130:841-848. 
Van Denderen BJ, Salvaris E, Romanella M, et al. Combination of decayaccelerating factor expression and alpha1,3-galactosyltransferase knockout affords added protection from human complement-mediated injury. Transplantation 1997;64 (6):882-888.

Varki A. Loss of N-glycolylneuraminic acid in humans: mechanisms, consequences and implications for hominid evolution. Am J Phys Anthropol. 2001;33:54-69.

Vaziri H, Benchimol S. Reconstitution of telomerase activity in normal human cells leads to elongation of telomeres and extended replicative life span. Curr Biol 1998;8:279-282.

Vega A, Garcia-Alonso D, Ramos A, et al. Immunohistochemical study of experimental acute cellular rejection. Transplantation Proc. 2002;34:731-732.

Vial CM, Ostlie DJ, Bhatti FN, et al. Life supporting function for over one month of a transgenic heart in a baboon. J Heart Lung Transplant 2000;19:224-229.

Vissel B, Choo KH. Four distinct alpha satellite subfamilies shared by human chromosomes 13, 14 and 21. Nucleic Acids Res 1991;19:271-277.

Walport MJ. Complement. First of two parts. N Engl J Med. 2001;344(14):10581066.

Wang G, Nonaka M, He C, et al. Functional differences among multiple isoforms of guinea pig decay-accelerating factor. J. Immunol. 1998;160:3014-3022.

Wang H, VerHalen J, Madariaga ML, et al. Attenuation of phagocytosis of xenogeneic cells by manipulating CD47. Blood 2007;109:836-842.

Wang N, Lee JM, Tobiasch E, et al. Induction of xenograft accommodation by modulation of elicited antibody responses. Transplantation 2002;74:334-345.

Wenzel K, Zabojszcza J, Carl M, et al. Increased Susceptibility to Complement Attack due to Down-Regulation of Decay-Accelerating Factor/CD55 in DysferlinDeficient Muscular Dystrophy. J Immunol. 2005;175:6219-6225.

Wheeler DG, Joseph M, Mahamud SD, et al. Transgenic swine: Expression of human CD39 protects against myocardial injury. J Mol Cel Cardiol 2012;52:958961.

Wilhite T, Ezzelarab C, Hara H, et al. The effect of Gal expression on pig cells on the human T-cell xenoresponse. Xenotransplantation 2012;19:56-63.

Willadsen SM. Nuclear transplantation in sheep embryos. Nature 1986;320:6365. 
Wilmut I, Schnieke AE, McWhir J, et al. Viable offspring derived from fetal and adult mammalian cells. Nature 1997;385:810-813.

Wuensch A, Klymiuk N, Kurome M, et al. Expression of Human Thrombomodulin on the Endothelium of Pig Xenograft Donors. Xenotransplantation 2012;94:787.

Yang YG, Sykes M. Xenotransplantation: current status and a perspective on the future. Nat Rev Immunol 2007;7(7):519-531.

Yamada K, Sachs D, DerSimonian H, et al. Human anti-porcine xenogeneic T cell response. Evidence for allelic specifity of mixed leukocyte reaction and for both direct and indirect pathways of recognition. J Immunol 1995;155:52495256.

Yannoutsos N, Langford GA, Cozzi E, et al. Production of pigs transgenic for human regulators of complement activation. Transplant Proc 1995;27:324-325.

Yeom HJ, Koo OJ, Yang J, et al. Generation and Characterization of Human Heme Oxygenase-1 Transgenic Pigs. PLOS One 2012;7(10):e46646.

Yi S, Hawthorne WJ, Lehnert AM, et al. T cell-activated macrophages are capable of both recognition and rejection of pancreatic islet xenografts. J. Immunol. 2003;170:2750-2758.

Zakian VA. Telomeres: beginning to understand the end. Science 1995;270:1601-1607.

Zambrowicz BP, Imamoto A, Fiering S, et al. Disruption of overlapping transcripts in the ROSA beta geo 26 gene trap strain leads to widespread expression of beta-galactosidase in mouse embryos and hematopoietic cells. Proc Natl Acad Sci USA 1997;94:3789-3794.

Zhen-Wei X, Jian-Le S, Qi Q, et al. Heme oxygenase 1 improves the survival of discordant cardiac xenograft through its anti-inflammatory and anti-apoptotic effects. Pediatr Transplant 2007;11:850-859.

Zhou CY, McInnes E, Copeman L, et al. Transgenic pigs expressing human CD59, in combination with human membrane cofactor protein and human decayaccelerating factor. Xenotransplantation 2005;12:142-148. 
10. Supplementay part: Primer sequences

\begin{tabular}{|c|c|}
\hline $\mathrm{AmF}$ & TCCCTTAACGTGAGTTTTCGTT \\
\hline BemF & CATGCTCACGGCAATGCCGG \\
\hline CD39 Ex5F & AGACAGGGTTCTGGATGTGG \\
\hline CD39 Ex6R & CAAGGTCCAAAGCTCCAAAG \\
\hline $\mathrm{CD} 46$ 2cF & TTGTGATCGGAATCATACATGGC \\
\hline CD46 2nF & TGTCTCAGATGACGCCTGTT \\
\hline CD46 3cR & AGTGCATCTGATAACCAAACTCG \\
\hline CD46 miniPCR2F & ACTCAGCTGGAATGTTACACC \\
\hline CD46 miniPCR2R & СTTATCTGGTTGCGGCCGCGATAACCAAACTCGTAAGTCC \\
\hline CD46 miniPCR3F & CTTTAAATGGCCAAGCAGTCC \\
\hline CD46 miniPCR3R & CGGATGCTAACTCGAGTCCTTCTCAGAGAGAAGTAAAT \\
\hline CD46 miniPCR1ePF & TGTGAATGGGTCGCGACCATCCGAATCCTTCTGCAT \\
\hline CD46 miniPCR1R & CAGACAGGATCCCTGCATTC \\
\hline CD55 CDNAApaIrev & TTCCGGAAGGGCCCCTAAGTC AGCAAGCCCATGGT \\
\hline CD55 Mini For2 & CAAATGTGAAGAAAGCTTTGTGA \\
\hline CD55 Mini N2 R & TCCTTCTCGCCAGGAATTTTCACA \\
\hline CD55 Mini 5 UTR Mlu & TGTGAATGGGACGCGTGGGCAACGTGCTGGTTATTG \\
\hline CD55 qPCR F2 & GGGCAGTCAATGGTCAGATAT \\
\hline CD55 qPCR R2 & ACGGCACTCATATTCCACAAC \\
\hline CD55 Va1F & TCAGCGCAACACGGTACCAGGCGGCGATCGCAATAGTT \\
\hline CD55 Va1R & ССTTATCTGGTTGGGCCCAGACCACCAGGCCTGTAATG \\
\hline CD55 VIaF & TCTGGACCCGTGATGGGCGGCCGCTCAACACGCAGAGGTGGTAA \\
\hline CD55 VIaR & CGGATGCTAACCGCGGATCCTGCGATCGCATTCATA \\
\hline CD55 7cF & CAGTAAATGTTCCAACTACAGAAG \\
\hline CD55 8cR & AACAGGTGTACTCCGTGTTGC \\
\hline CD59 3cF & GGAGTTGAGACCTACTTCACAG \\
\hline DAF F1 & GGATTCACCATGATTGGAGAGCACTC \\
\hline DAF R1 & AAGTCAGCAAGCCCATGGTTACTAGC \\
\hline hA20 7cF & TGGGACTCCAGAAAACAAGG \\
\hline hA20 8cR & GTCCTTTTGGCCTCATGAAA \\
\hline HBXIP F1 & GCCACGAACTGAAGCACTGAG \\
\hline HBXIP R1 & TGACAGGCTGCTGAAGAACAGA \\
\hline hHO Ex1 F1 & GATGGAGCGTCCGCAACC \\
\hline $\mathrm{HMOX3cF}$ & CTGTCTACTTCCCAGAAGAGC \\
\hline HMOX3cR & CTTCACATAGCGCTGCATGGC \\
\hline HMOX NF & GCCCCAGGATTTGTCAGAGG \\
\hline HMOX NR & TAAGGACCCATCGGAGAAGC \\
\hline hTert cDNA F1 & GCTGTCGGAAGCAGAGGTCA \\
\hline hTert cDNA R1 & GTTTGCGACGCATGTTCCTC \\
\hline Lea expr2R & ATGAGCTCCACCTTGCAGAT \\
\hline Lea $\mathrm{F}$ & TGACAGCCAGGTGACTGAAG \\
\hline Lea HAIFKpnI & AGTCTAGGTACCAAGTGTTCGGCCTCTCAAAG \\
\hline
\end{tabular}




$\begin{array}{ll}\text { Lea HAIRApaI } & \text { TAGACTGGGCCCCTTTCCTGGGATTCCAGCTT } \\ \text { Lea HAIIFNotI } & \text { AGTCTAGCGGCCGCCCTTCACAGACACACCCAGA } \\ \text { Lea HAIIRSacII } & \text { TAGACTCCGCGGCGAAGATCACACACCACCACAC } \\ \text { P BS F } & \text { CCCGTCCTGTGGATCTACCC } \\ \text { P BS 2 F } & \text { GCAGACACTCTATGCCTGTGTGG } \\ \text { P BS 2 R } & \text { TCTACCGGCAGTGCAAATCC } \\ \text { P EGFP R } & \text { AATGGGGCGGAGTTGTTACG } \\ \text { PL452 4R } & \text { AATTTCCATTCGCCATTCAG } \\ \text { PL452 3shF } & \text { GGATCAATTCTCTAGAGCTCGCTGA } \\ \text { PoCeM1 F } & \text { CTAGCTAGGTCCCTGCCGAGCTG } \\ \text { PoCeM1 R } & \text { GGTCTCAGACAGGGCACAAAAC } \\ \text { poGAPDH F1 } & \text { GGCGTGAACCATGAGAAGTATG } \\ \text { poGAPDH R1 } & \text { GGTGCAGGAGGCATTGCT } \\ \text { poHPRT F1 } & \text { GTGATAGATCCATTCCTATGACTGTAGA } \\ \text { poHPRT R1 } & \text { TGAGAGATCATCTCCACCAATTACTT } \\ \text { Rosa26 I1 F2 } & \text { TATGGGCGGGATTCTTTTGC } \\ \text { Rosa26 I1R3 } & \text { GTTTGCACAGGAAACCCAAG } \\ \text { Rosa26 Loc 3R } & \text { TCCGAAGCCCAACCTTTCAT } \\ \text { Rosa26 Loc 2R } & \text { GGAGCGGCGATACCGTAAAG } \\ \text { Rosa26 Loc 2R } & \text { GGAGCGGCGATACCGTAAAG } \\ \text { sDAF R } & \text { AGGAGTTCGAGACTGCAGTGAGCTACGATCACAC } \\ \text { SpecNheIF } & \text { CGATCCGCTAGCTCTAGACCAGCCAGGACAGAA } \\ \text { SpecRsrIIR } & \text { TTTTTTCGGTCCGCGAGGGTTATTTGCCGACTA } \\ \text { SRosa26 R1 } & \text { CGCCTAGAGAAGAGGCTG } \\ \text { TMncF } & \text { AGGACGTGGATGACTGCATAC } \\ \text { TMncR } & \text { TACTCGCAGTTGGCTCTGAAG } \\ \end{array}$




\section{Acknowledgement}

Ganz herzlich möchte ich mich zuerst bei Angelika Schnieke bedanken. Sie hat mir die Möglichkeit gegeben meine Promotion am Lehrstuhl durchzuführen. Ich hatte immer sehr viel Spaß dabei mit ihr zusammen zu arbeiten und mein Projekt weiter $\mathrm{zu}$ entwickeln. Sie hat mir gezeigt wie man die eigenen Projektziele erreichen kann und gleichzeitig Alternativen einplanen sollte um weiter voran zu kommen. Für ihre Arbeit und ihren Einsatz für mich und auch für alle anderen bin ich ihr sehr dankbar.

Mein Dank gilt auch Alex Kind, der sich ebenfalls so sehr für jeden einzelnen einsetzt. Ohne ihn wäre es kaum möglich alle Publikationen und Anträge in dieser Form zu erstellen. Auch für mich und meine Arbeiten hat er viel Zeit investiert um ihnen den letzten Schliff zu verleihen. Thanks a lot, Alex!

Auch bei Simone Kraner-Scheiber möchte ich mich herzlichst bedanken. Sie hat meine Arbeit betreut, war immer da wenn es Probleme gab und hat mir den Freiraum gelassen um Verantwortung zu übernehmen und mein Projekt selbstständig zu planen. Ohne sie wären all diese Ergebnisse nicht möglich gewesen.

Sulith Christan möchte ich danken, dass sie sich immer um alles kümmert, dass sie nie genervt ist auch wenn man 10-mal am Tag zu ihr kommt und ganz dringend etwas braucht, dass sie immer alles organisiert und so hilfsbereit ist. Vielen Dank für alles!

Bei Marlene Edlinger möchte ich mich auch herzlichst bedanken. Sie hat mir am Anfang viele Methoden gezeigt und mich gerade zu Beginn meiner Arbeit sehr stark unterstützt. Vielen Dank, Leni !

Auch bei allen anderen TAs möchte ich mich bedanken. Mein Dank gilt Margret Bahnweg, Peggy Müller-Fliedner und Christina Mosandl für die angenehme Zusammenarbeit. Besonders möchte ich mich bei Kilian Skowranek und Toni Kuhnt bedanken. Beide haben mehrere Jahre eng mit mir zusammen gearbeitet und tolle Ergebnisse dabei erzielt. Und Toni: Morgen gibt's wieder was zu präpen ;)

Auch Barbara Bauer gilt mein Dank. Sie organisiert so viel und sorgt immer dafür, dass alles funktioniert. Danke Bärbel ! 
Steffen und Viola Löbnitz möchte ich für ihren Einsatz, die gute Zusammenarbeit, das ständige Beschaffen von Probenmaterial und die Pflege unserer Tiere danken. Krzysztof Flisikowski und Tatiana Flisikowska möchte ich für die gute Zusammenarbeit, ihre stetige Gastfreundschaft und die schöne Zeit in Polen danken.

Auch bei meinen beiden Bachelorstudenten Carolin Willberg und Eva Siebert möchte ich mich bedanken. Besonders mit Eva hatte ich so viel Spaß im Labor und wir mussten so viel lachen. Auch bei meinen Masterstudenten möchte ich mich herzlichst bedanken. Christina Kersten, Mona Baumgart, Lisa Jordan, Melanie Thiessen, Lara Riehl, Nina Simm, Andrea Schäffler und Beate Rieblinger haben dazu beigetragen die Forschung im Bereich der Xenotransplantation weiter voran zu bringen. Ihnen allen wünsche ich alles Gute für ihre Zukunft.

Besonders Andrea Schäffler wünsche ich alles Gute für ihre zukünftige Promotion bei uns am Lehrstuhl. Allen Promotionsstudenten, besonders jedoch Daniela Fellner, Carolin Wander, Benedikt Baumer, Beate Rieblinger und Erica Schulze möchte ich für die schöne Zeit am Lehrstuhl und die schönen Abende danken. Ich habe mich immer sehr gefreut so aktiv an ihren Projekten teilzunehmen.

Ganz besonders danke ich Erica Schulze für die lustige Zeit, ihre stetige Gastfreundschaft, die Blumen, ihre enge Freundschaft, die gemeinsamen Feiern sowie das gemeinsame Kochen. „Wir" machen das schon ;)

Besonders Beate Rieblinger möchte ich noch alles Gute für den letzten Abschnitt ihrer Promotion wünschen. Es hat mir jeden Tag sehr viel Spaß gemacht zusammen mit ihr zu arbeiten. Ich danke ihr für ihre Arbeit, ihre Hilfe und für die sehr enge Freundschaft. Ohne Bea wäre meine Promotion bei weitem nicht so schön gewesen.

Mein größter Dank gilt meinen Eltern. Durch ihre tägliche, harte und unermüdliche Arbeit haben sie mir erst die Möglichkeit gegeben diese Promotion durchzuführen und mir gezeigt wie wichtig es ist sich für seine Ziele einzusetzen und niemals aufzugeben. Danke euch beiden! 


\section{Curriculum Vitae}

\section{Persönliche Daten}

Name: Konrad Josef Fischer

Geburtsdatum: 04.03.1983

Geburtsort: Augsburg, Deutschland

\section{Ausbildung}

02/2010-12/2015 Promotionsstudium, Molekulare Biotechnologie Technische Universität München Thesis: "Multi-transgenic pigs for xenotransplantation"

10/2007-02/2010 Master of Science, Molekulare Biotechnologie Technische Universität München Abschlussnote: 1,1 Thesis: "Reaktionstechnische Charakterisierung und Metabolomanalyse von rekombinanten Hefen zur Produktion von Bernsteinsäure“

10/2004-09/2007 Bachelor of Science, Molekulare Biotechnologie Technische Universität München Abschlussnote: 1,3 Thesis: "Einfluss von N-terminaler Acylierung auf membranfusogene Modellpeptide im Hinblick auf Liposomenfusion, Struktur und Oligomerisierungsverhalten“

07/2003-03/2004 Zivildienst Kreiskrankenhaus Friedberg Abteilungen Notfallambulanz und Endoskopie

1994-06/2003 Wernher von Braun Gymnasium Friedberg Allgemeine Hochschulreife Abschlussnote: 1,0

\section{Veröffentlichungen und Präsentationen}

\section{Peer-reviewed Paper}

Dutta R, Li S, Fischer K, Kind A, Flisikowska T, Flisikowski K, Rottmann O, Schnieke A. Non-invasive assessment of porcine oocyte quality by supravital staining of cumulus-oocyte complexes with lissamine green B. Zygote 2015:1-10. 
Poschner BC, Fischer K, Herrmann JR, Hofmann MW, Langosch D. Structural features of fusogenic model transmembrane domains that differentially regulate inner and outer leaflet mixing in membrane fusion. Mol Membr Biol. 2010;27(1):1-10.

\section{Other publications}

Rieblinger B, Fischer K, Kraner-Scheiber S, Flisikowska T, Petersen B, Buermann A, Schwinzer R, Niemann H, Kind A, Schnieke A. Modifying pigs to overcome barriers to xenotransplantation. IXA Conference Paper 2015.

Morozov V, Poltzki E, Heinrichs G, Wolf-van Buerck L, Knauf Y, Becker T, MaetzRensing K, Schuster M, Baehr A, Klymiuk N, Wolf E, Seissler J, Morozov AV, Rotem A, Barkai U, Bornstein S, Fischer K, Schnieke A, Petersen B, Niemmann H, Abicht JM, Güthoff S, Reichart B, Denner J. Microbiological screening of wild-type and genetically engineered pigs and transplant recipients in pig to non-human primate xenotransplantation. IXA Conference Paper 2015.

Fischer K, Kraner-Scheiber S, Flisikowska T, Schnieke A. Effective combination of xenoprotective transgenes. Xenotransplantation 2014;21:191.

Fischer K, Kraner-Scheiber S, Flisikowski K, Flisikowska T, Schnieke A. Multitransgenic pigs for xenotransplantation. Xenotransplantation 2013;20:64.

\section{Vorträge}

09/2015 Presentation of proceedings project B2, Xenotransplantation, Deutsche Forschungsgemeinschaft SFB TRR 127

04/2015 Presentation of proceedings project B2, Xenotransplantation, Deutsche Forschungsgemeinschaft SFB TRR 127

11/2014 "Multi-transgenic pigs and the basics of xenotransplantation" WZW Evaluierung, Freising, Germany

10/2014 Presentation of proceedings project B2, Xenotransplantation, Deutsche Forschungsgemeinschaft SFB TRR 127

04/2014 Presentation of proceedings project B2, Xenotransplantation, Deutsche Forschungsgemeinschaft SFB TRR 127

10/2013 "Combining xenoprotective transgenes" International Xenomeeting, San Servolo, Italy

03/2011 Presentation of proceedings, Deutsche Forschungsgemeinschaft, SFB Xenotransplantation

10/2010 Presentation of proceedings, Deutsche Forschungsgemeinschaft, SFB Xenotransplantation 
Posterpräsentationen

03/2016 Genetically modified pigs to modulate vascular and cellular rejection. SFB TRR 127. Dresden, Germany

03/2016 Identification and characterization of new xenoprotective transgenes. SFB TRR 127. Dresden, Germany

12/2015 Modifying pigs to overcome barriers to xenotransplantation. Salaam Meeting. Poznan, Poland

07/2012 Multi transgenic pigs for xenotransplantation. Meeting Xenotransplantation. Berlin, Germany

03/2012 Pigs deficient in the major xenoantigen non-Gal antigen Neu5Gc. SFB TRR 127. Munich, Germany 